\title{
Soot formation in laminar counterflow flames
}

\author{
Yu Wang ${ }^{1, *}$, Suk Ho Chung ${ }^{2, *}$
}

${ }^{1}$ School of Automotive Engineering, Wuhan University of Technology, Wuhan, P.R. China

${ }^{2}$ Clean Combustion Research Center (CCRC), King Abdullah University of Science and Technology (KAUST), Thuwal, Saudi Arabia

* Corresponding authors

Yu Wang

School of Automotive Engineering

Wuhan University of Technology

Wuhan, P.R. China

Email: yu.wang@,whut.edu.cn

Suk Ho Chung

Clean Combustion Research Center

King Abdullah University of Science and Technology

Thuwal, Saudi Arabia

Email: sukho.chung5@gmail.com

Review article

Prepared for submission to Progress in Energy and Combustion Science 


\title{
Soot formation in laminar counterflow flames
}

\author{
Yu Wang ${ }^{1, *}$, Suk Ho Chung ${ }^{2, *}$ \\ ${ }^{1}$ School of Automotive Engineering, Wuhan University of Technology, Wuhan, P.R. China \\ ${ }^{2}$ Clean Combustion Research Center (CCRC), King Abdullah University of Science and \\ Technology (KAUST), Thuwal, Saudi Arabia
}

\begin{abstract}
Many practical soot-emitting combustion systems such as diesel and jet engines rely on diffusion flames for efficient and reliable operation. Efforts to mitigate soot emissions from these systems are dependent on fundamental understanding of the physicochemical pathways leading from fuel to soot in laminar diffusion flames. Existing diffusion flame-based soot studies focused primarily on overventilated coflow flame where the fuel gas (or vapor) issues from a cylindrical tube into a co-flowing oxidizer, and counterflow flame, where a reacting zone is established between two opposing streams of fuel and oxidizer. As a canonical diffusion flame configuration, laminar counterflow diffusion flames have been widely used as a highly controllable environment for soot research, enabling significant progress in the understanding of soot formation for several decades. In view of the possibility of fuel/oxidizer premixing in practical systems, counterflow partially premixed flames have also been studied. In the present work we intend to provide a comprehensive review of the researches on various aspects of soot formation utilizing counterflow flames. Major processes of soot formation (formation of gas phase soot precursors, soot inception and surface reactions, as well as particle-particle interactions) are examined first, with focus on the most recent (post-2010) research progress. Experimental techniques and associated challenges for the measurement of soot-related properties (some knowledge of which is helpful for full appreciation of the experimental data to be reviewed) are then introduced. This is followed by a detailed description of soot evolution in counterflow flames, which is complemented by a discussion on the similarity and differences of the sooting structures between counterflow and coflow diffusion flames. Parametric studies of the effects of fuel molecular structure, fuel additive, partial-premixing, pressure, temperature, stoichiometric mixture fraction, and residence time on soot formation in counterflow flames will then be addressed in detail. This review concludes with a summary of the knowledge and challenges gathered and demonstrated through decades of research, and an outlook on opportunities for future counterflow flame-based soot research towards a more complete understanding of soot formation and the development of novel techniques for soot mitigation in practical combustion devices.
\end{abstract}




\section{Contents}

1. INTRODUCTION

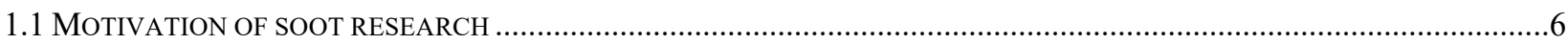

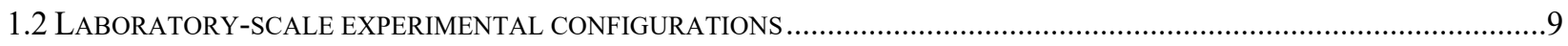

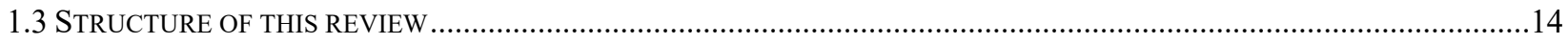

2. PATHWAYS OF SOOT FORMATION …...............................................................................................................15

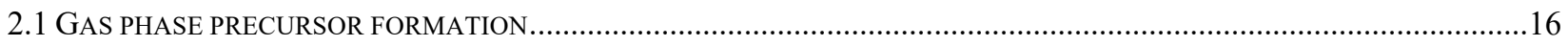

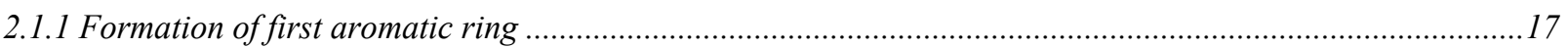

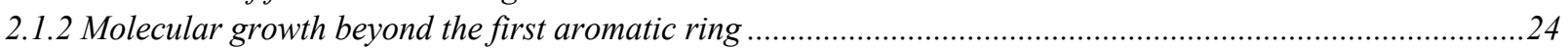

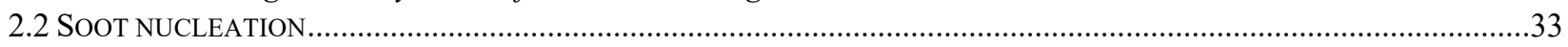

2.3 SOOT SURFACE GROWTH, PARTICLE COALESCENCE, AND AGGLOMERATION........................................................42

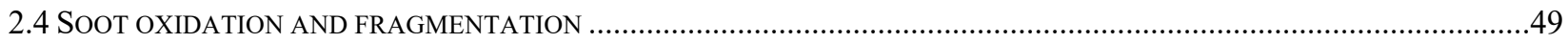

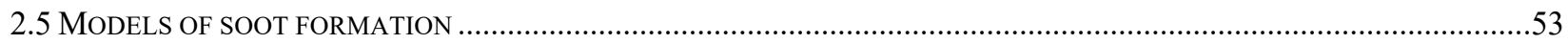

3. EXPERIMENTAL TECHNIQUES FOR SOOT RELATED MEASUREMENTS...............................................57

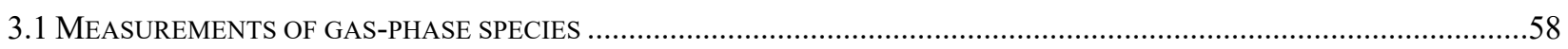

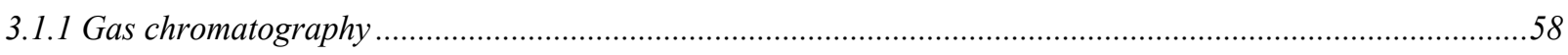

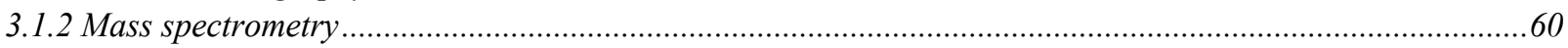

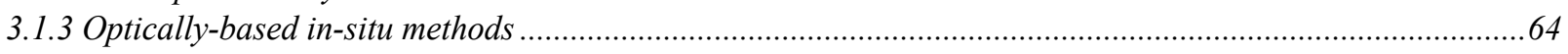

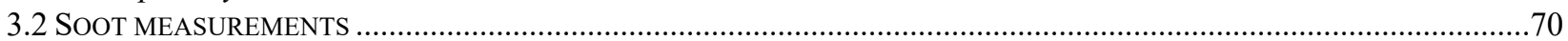

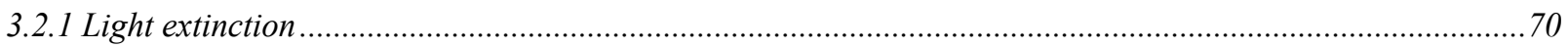

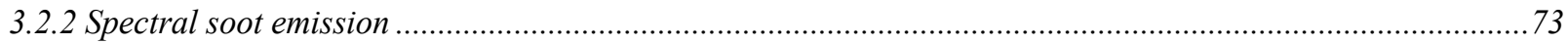

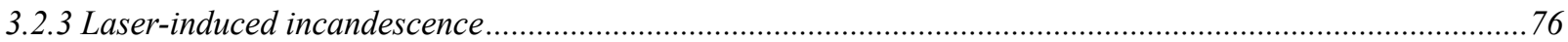

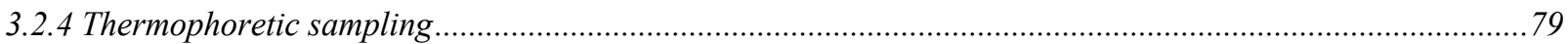

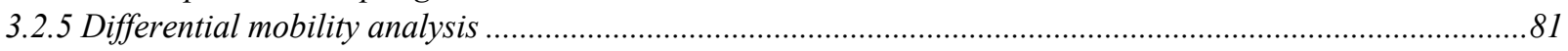

4. SOOTING STRUCTURES AND SOOT EVOLUTION IN COUNTERFLOW FLAMES....................................84

4.1 SOOTING STRUCTURES OF CDFS AND COMPARISON WITH COFLOW DIFFUSION FLAMES ..........................................84

4.2 INFLUENCES OF STOICHIOMETRIC MIXTURE FRACTION ON SOOTING STRUCTURES IN CDFS ...................................92

4.3 INFLUENCES OF STRAIN RATE ON SOOTING STRUCTURES IN CDFS...................................................................97

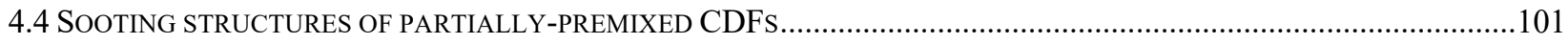

5. SOOT FORMATION IN COUNTERFLOW DIFFUSION FLAMES: PARAMETRIC STUDIES ....................103

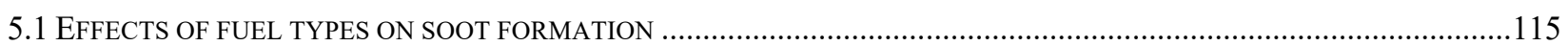

5.1.1 Counterflow diffusion flames with single component (neat) fuels.............................................................116

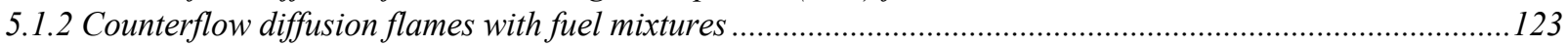

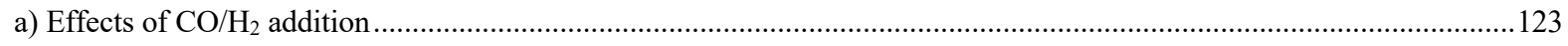

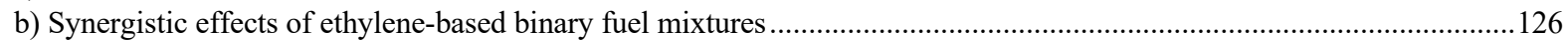

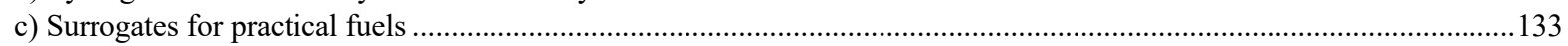

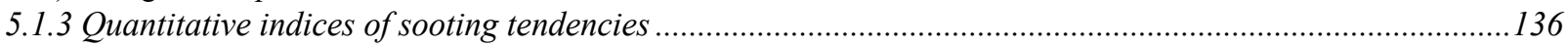

5.2 EFFECTS OF DILUTION AND NON-HYDROCARBON ADDITIVES ON SOOT FORMATION ……….................................143

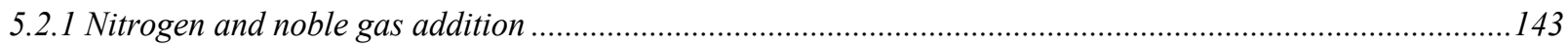

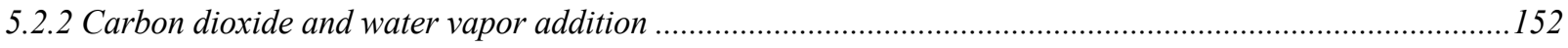

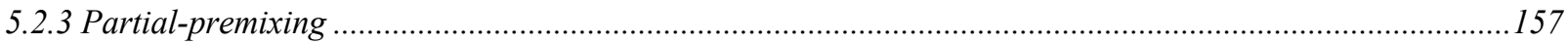

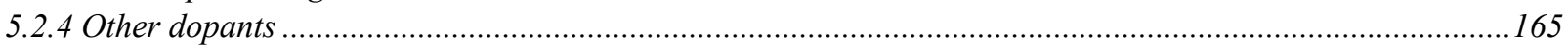

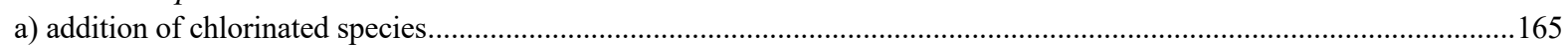

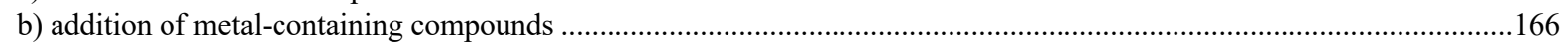

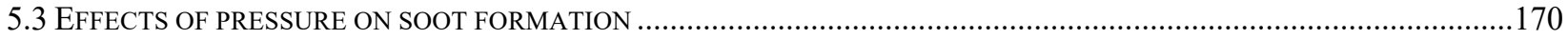

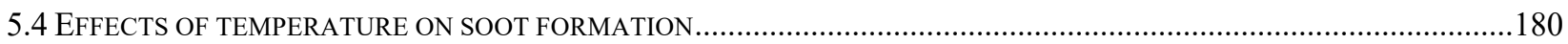

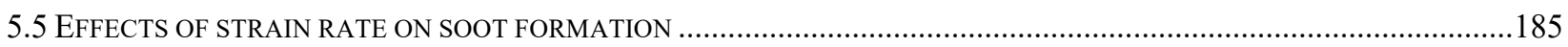

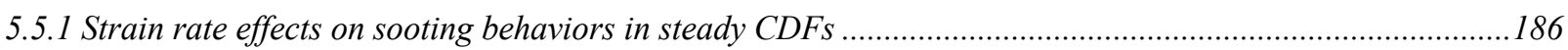

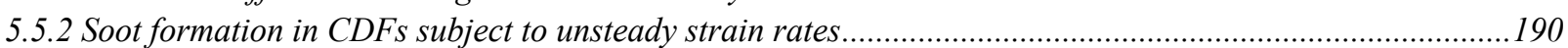

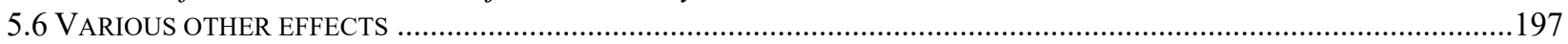

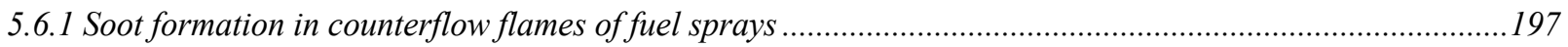


5.6.2 Effects of electric fields on soot formation in CDFs

5.6.4 Interactions between soot and nitric oxides ...

6. SUMMARY, CHALLENGES, AND PROSPECTS....................................................................................205 


\begin{tabular}{|c|c|c|c|}
\hline \multicolumn{4}{|c|}{ Nomenclature } \\
\hline \multicolumn{4}{|c|}{ Abbreviations } \\
\hline $\mathrm{AC}$ & alternative current & PAC & phenyl addition/cyclization \\
\hline AFM & atomic force microscope & PAH & polycyclic aromatic hydrocarbon \\
\hline ARS & aromatic site & PIE & photo-ionization efficiency \\
\hline CARS & coherent anti-stokes Raman spectroscopy & PM & particulate matter \\
\hline CDF & counterflow diffusion flame & PMT & photomultiplier tube \\
\hline CI & compression ignition & PPF & partially-premixed flame \\
\hline $\mathrm{CHC}$ & chlorinated hydrocarbons & PSD & particle size distribution \\
\hline $\mathrm{CPC}$ & condensation particle counter & REMPI $r$ & resonance-enhanced multi-photon ionization \\
\hline CRDS & cavity ring-down spectroscopy & RSR 1 & resonantly stabilized radical \\
\hline DFT & density functional theory & SEM & scanning electron microscopy \\
\hline DMA & differential mobility analysis & SF & soot formation \\
\hline EI & electron ionization & SFO & soot formation/oxidation \\
\hline EXP & experiment & SI & spark ignition \\
\hline FBC & fuel-borne catalyst & SIM & simulation \\
\hline FFT & fast Fourier transform & SMD & Sauter mean diameter \\
\hline FID & flame-ionization detector & SMPS & scanning mobility particle sizer \\
\hline FTIR & Fourier transform infra-red spectroscopy & SP & smoke point \\
\hline GC & gas chromatography & SSE & spectral soot emission \\
\hline GDI & gasoline direction injection & SSI & sooting sensitivity index \\
\hline НАB & height above burner & STI & sooting temperature index \\
\hline HACA & hydrogen-abstraction-acetylene-addition & SVF & soot volume fraction \\
\hline HIM & helium-ion microscopy & TCD & thermal conductivity detector \\
\hline IC & internal combustion & TEM & transmission electron microscopy \\
\hline ICCD & intensified charge coupled device & TGA & thermal-gravimetric analysis \\
\hline IR & infrared & TOF & time-of-flight \\
\hline JSR & jet-stirred reactor & TR & time-resolved \\
\hline KMC & kinetic Monte Carlo & TSI & threshold soot index \\
\hline LAS & laser absorption spectroscopy & UHC & unburned hydrocarbon \\
\hline LDV & laser doppler velocimetry & VOA & vibratory orifice atomizer \\
\hline LE & light extinction & UV & ultra-violet \\
\hline LE/LS & light extinction and scattering & VUV & vacuum ultra-violet \\
\hline LIE & laser-induced emissions & TDLAS t & tunable diode laser absorption spectroscopy \\
\hline LIF & laser-induced fluorescence & TSI & threshold soot index \\
\hline LII & laser-induced incandescence & XRD & X-ray diffraction \\
\hline MAC & methyl addition/cyclization & YSI & yield soot index \\
\hline MB & molecular beam & RDG-FA & A Rayleigh-Debye-Gans fractal aggregate \\
\hline MBMS & molecular beam mass spectrometry & & \\
\hline MPI & micropyrolysis index & & \\
\hline MS & mass spectrometry & & \\
\hline NOC & nanoscale organic carbon particle & & \\
\hline NOx & nitic oxides & & \\
\hline NSP & normalized smoke point & & \\
\hline OESI & oxygen extended sooting index & & \\
\hline
\end{tabular}




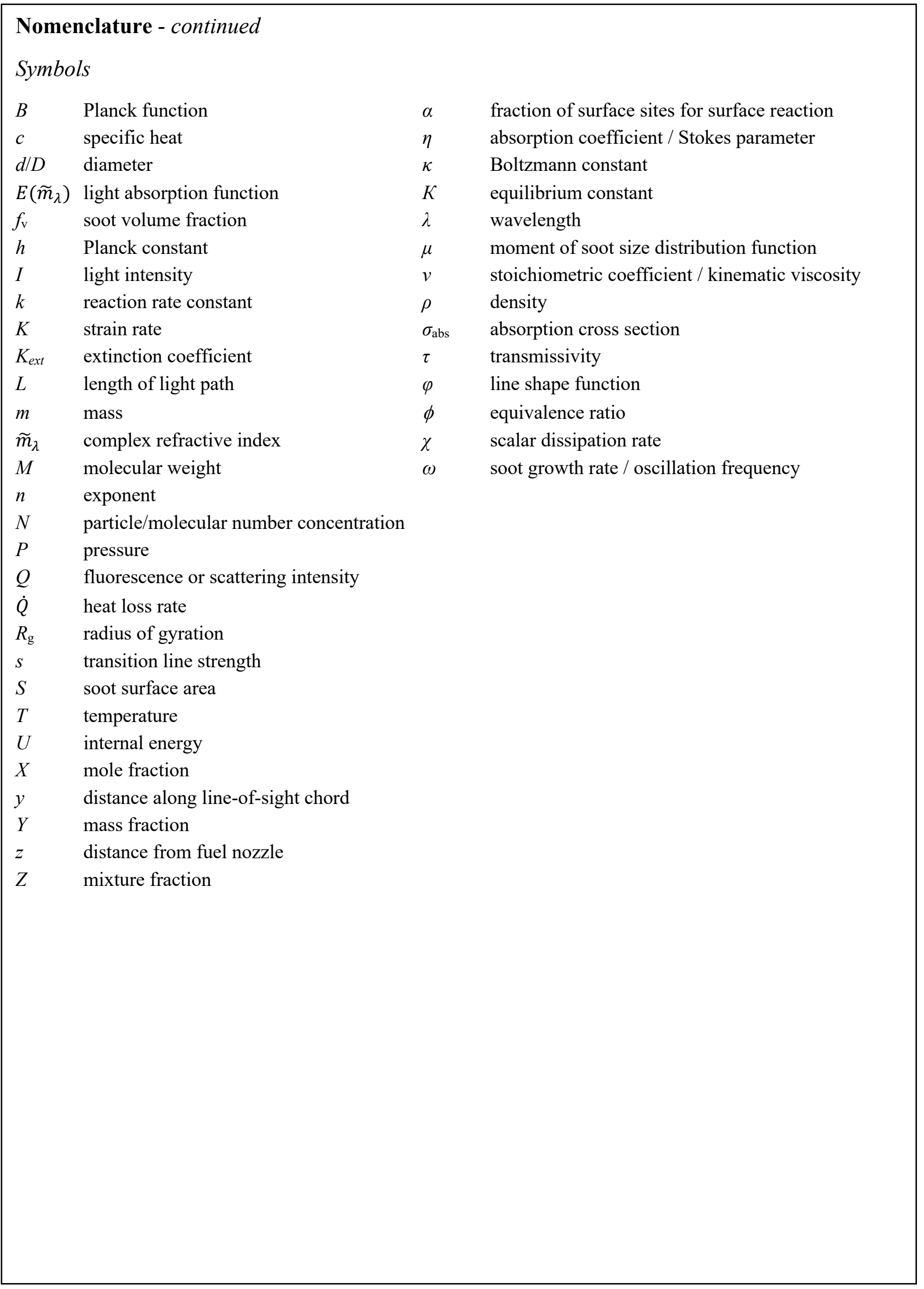




\section{Introduction}

\subsection{Motivation of soot research}

Rapid population growth and progressive industrialization of developing countries assure rising worldwide energy demands in the coming decades: A 30\% increase in energy consumption is projected from 2020 to 2040, even though continuous improvement in efficiency of energy use is anticipated [1]. Although alternative sources such as wind, solar, geothermal, and nuclear energies are increasingly important in the world energy mix, conventional petroleum-based fuels are still expected to play a dominant role in the foreseeable future. This is especially true in the transportation sector, where the society relies heavily on internal combustion (IC) engines fueled by natural gas, gasoline, kerosene, and diesel. Note that IC engines use $70 \%$ of the 86 million barrels of crude oil the world consumes every day [2]. Energy from hydrocarbons comes with a price. Apart from contributing to the greenhouse effects and thus to climate change, the use of hydrocarbon fuel also leads to the formation of various hazardous pollutants, including-but not limited to-nitric oxide (NOx), carbon monoxide (CO), unburned hydrocarbons (UHCs) and soot. Here soot is defined as carbonaceous particles resulting from pyrolysis or incomplete combustion of hydrocarbon fuels. In terms of elemental composition, soot is largely carbon and may also contain small amounts of hydrogen and oxygen. Being very fine particles with diameters typically less than a micrometer, soot is known to be an important contributor to $\mathrm{PM}_{2.5}$ (particulate matter with diameters smaller than 2.5 $\mu \mathrm{m})$.

As far as reciprocating IC engines are concerned, NOx, $\mathrm{CO}$, and $\mathrm{UHC}$ can be effectively removed using a three-way catalyst in spark-ignition (SI) engines operating with stoichiometric fuel-air mixtures. Soot emission is generally not an issue in these conventional port-injection SI engines. But with the advent of gasoline direct injection (GDI) technology (intended mainly to improve fuel efficiency), soot particles can be produced at locally rich regions as a result of charge inhomogeneity. 
Although the total soot mass emitted from GDI engines may not be significant, its number density can still be tremendous, since GDI engines are likely to produce very fine soot particles [3, 4]. Compression ignition (CI) diesel engines are conventional producers of NOx and soot, and worse, a trade-off relationship exists between NOx and soot formation in the diesel combustion process. Many measures designed to reduce NOx emission result in inhibiting the oxidative removal of soot, signifying the importance of soot suppression during its formation stage. As environmental regulations on soot emission become increasingly stringent, the incentive for combustion scientists and engineers to develop innovative and effective methods for soot mitigation grows with it.

Concerns about soot emission are many and justified. Being the major condensed-phase by-product, soot originates from the incomplete combustion of hydrocarbon fuels. In most cases, the presence of soot indicates poor combustion conditions, where not all the fuel molecules can be fully oxidized to form $\mathrm{CO}_{2}$ and $\mathrm{H}_{2} \mathrm{O}$ for maximum thermal energy recovery. Soot particles contain chemical energy in the form of $\mathrm{C}-\mathrm{H}$ and $\mathrm{C}-\mathrm{C}$ bonds, which could be released as desirable thermal energy if soot formation could be avoided altogether, or if all the initially formed soot particles were fully oxidized in a later stage. Therefore, the emission of soot from practical combustion devices can be translated directly to combustion inefficiency.

More importantly, soot emission into the atmosphere has dire consequences to human health and the environment. After being inhaled, ultrafine soot particles with diameters less than $100 \mathrm{~nm}$ (typical for flame generated soot) can travel deeply into the lungs, deposit in the alveoli, and cross the cells to enter the circulatory system [5]. These ultrafine particles can then be translocated to other organs like the liver, heart, and brain [6], causing potential health issues such as cardiovascular diseases. Polycyclic aromatic hydrocarbons (PAHs), the main organic component of soot, have also been associated with carcinogenic and mutagenic effects [7]. Schwartz and coworkers [8, 9] demonstrated positive correlations between PM concentrations and mortality rates that reflect the serious health issues caused by soot emission. 
Regarding environmental implications, soot is a major contributor to global warming, second only to $\mathrm{CO}_{2}$ [10]. Such efficacy is believed to be caused by its influence on snow albedo as well as its atmospheric stability at high altitudes [11]. Similarly, soot deposited on ice glaciers can promote melting, raising the sea level with all the accompanying problems. Soot particles, with graphitic carbon as the primary constituent, are the biggest contributor to light absorption by atmospheric aerosols [12]. The reduction of visibility in densely populated urban areas due to the presence of soot could cause widespread societal concerns. Recent severe haze events in China, affecting nearly 800 million people, have been closely linked to fossil fuel use. It has been suggested that both primary particulate and secondary aerosol precursors from fossil fuel and biomass combustion can be controlled to minimize the negative impacts [13]. Because of its contribution as cloud condensation nuclei, soot in the upper atmosphere is also known to affect weather in the form of clouds and precipitation [14-16].

In most cases, soot is the unwanted by-product of hydrocarbon combustion and its formation needs to be suppressed as much as possible. However, there are situations in which soot production is intentional. In utility and industrial boilers, for example, released combustion heat needs to be effectively transferred to the feedwater. One important heat transfer mode in such large-scale systems is radiation, which can be significantly enhanced by the presence of soot particles [17]. Of course, these particles need to be eliminated before the flue gases are emitted to the environment. In addition, carbon black is an important raw material for the reinforcing filler in tires and the industry strives for maximum soot yield for a given quantity of hydrocarbon fuel.

The need to suppress soot emission, or to produce soot with special properties, necessitates active control of the soot formation processes. This in turn requires a fundamental understanding of the physicochemical pathways from fuel to soot. Soot formation is one of the most complex phenomena in combustion, involving complicated interactions between combustion chemistry, fluid mechanics, mass/heat transport, and particle dynamics, so that despite decades of active research, many gaps still 
remain in our understanding of soot [18]. In the attempt to bridge these gaps, the underlying mechanisms of soot formation are the objectives of combustion scientists worldwide, evident from the large body of literature about soot published in recent years. For instance, soot, as a keyword, is present in the title or abstract of 45 articles published in Combustion and Flame in the year of 2015, accounting for more than ten percent of the 383 total articles. This number is even higher for 2016, with 51 out of the 374 articles investigating soot formation.

\subsection{Laboratory-scale experimental configurations}

A survey of the literature reveals that a significant portion of soot studies conducted to date are based on laboratory-scale laminar flames at atmospheric, or even lower pressures. This may seem inappropriate, at first glance, as combustion modes in most soot-emitting practical combustion devices are turbulent, and under high pressure. Such a mismatch is certainly not because soot formation in high-pressure turbulent flames can be well represented by those in laminar flames. One only needs to consider turbulence-chemistry interactions and the pressure-dependence of reaction rates to realize the drastic differences. However, the following facts limit our ability to investigate in detail the soot characteristics of high-pressure turbulent flames. First, the highly transient and inhomogeneous nature of turbulent flames presents a challenge for many soot-related experimental techniques in terms of providing sufficient temporal and spatial resolutions. Second, a high peak value and wide dynamic range of soot loadings prevent many high-pressure turbulent flames from being reliably diagnosed. Related issues include, for example, probe clogging for intrusive methods and signal attenuation/beam steering for optical methods [19]. Third, it is somewhat difficult to establish well-controlled high-pressure flames that are suitable for fundamental studies of soot formation and oxidation processes; several researchers have observed flame stability issues at high pressures [20, 21]. With an increase in pressure, the gas-solid conjugate heat transfer between flame gas and burner 
nozzle becomes increasingly important and complicates the specification of flow boundary conditions $[22]$.

In addition to the above technical constraints, the fact that physicochemical formation pathways of soot are still not fully understood-even in zero-dimensional systems-may provide enough rationale for the wide interests in soot studies with simple flow configurations. In this regard, various laboratory-scale setups have been employed, including constant volume combustion chambers [2330], shock tubes [31-40], well-stirred reactors [41-45], burner-stabilized flat premixed flames [4655], coflow diffusion flames [56-72], and counterflow diffusion flames (CDF) [73-92]. The conditions for soot formation can be very different among these experimental configurations and conclusions regarding the sooting processes obtained from one configuration may not be directly applicable to others. For example, the trend that soot loadings increase with the increase in flame temperature [93-96], as observed in diffusion flames, can be reversed in some premixed flames [9799]. This can be explained (at least partly) by noting that unlike in a diffusion flame, a very rich fuel pyrolysis region, where soot precursors are formed without oxidation, usually does not exist in a premixed flame. In addition, certain fuel additives may play different roles, sometimes with opposite effects on soot formation in different reacting systems. The doping of small amounts of ethanol, or dimethyl ether, was seen to boost soot volume fractions in ethylene diffusion flames [100, 101]; but it inhibited soot formation in ethylene/air premixed flames [102, 103].

Constant volume/pressure combustion chambers are typically used to study liquid fuel spray combustion [23-30], a close representation of the combustion process in CI engines. Unfortunately, soot formation during the burning of fuel sprays depends not only on soot chemistry but on many other physical processes such as spray penetration, droplet size distribution, and velocity field of the entrained air. It is challenging to isolate these factors to obtain quantitative information on soot behaviors. Note that a shock tube can provide homogeneous, constant pressure/temperature conditions, ideal for studying the chemistry of fuel pyrolysis and subsequent soot formation. However, 
the time scale of the sooting processes in shock tube experiments is typically $1 \sim 2$ orders of magnitude shorter than that in flames [31]. For this reason, shock tubes are primarily used for investigation of early stage soot formation; while slower processes such as particle coagulation and aggregation cannot be effectively examined [31]. A jet-stirred reactor (JSR) is an alternative way to reach constant pressure and temperature conditions, and the residence time can be varied by changing the reactants' flow rates. However, external heat sources are needed and typical temperature achievable in JSR experiments is only at the lower end of flame environments. Moreover, the reactants in shock tubes or JSR are highly diluted by inert gas (e.g., argon) in most cases, differing noticeably with practical flame conditions. Note that the effect of reactant dilution on soot formation can be significant [95, $104]$.

The details of soot formation in turbulent diffusion flames entail complex study, and most combustion systems rely on turbulent diffusion flames for operation. Fortunately, the concept of laminar flamelets could provide a link between turbulent and laminar diffusion flames [105, 106]. Fundamental investigations of the pathways from fuel molecules to soot particles in laminar diffusion flames can provide essential information toward clarifying the sooting process in practical flames.

Soot formation characteristics in laminar diffusion flames have been extensively studied in overventilated coflow diffusion flames, where the fuel gas (or vapor) issues from a cylindrical tube into co-flowing oxidizer [107], and counterflow flames, where a reaction zone is established between two opposing streams of fuel and oxidizer $[108,109]$. The general features of the flow configurations differ between these two types of flames. A coflow flame has a two-dimensional flow field so that the species concentrations, temperatures, and flow velocities vary in both axial and radial directions. A counterflow flame, on the other hand, is quasi-one-dimensional along the normal direction of the flame, a feature that significantly facilitates the analysis of flame/sooting structures. This becomes particularly relevant if models with detailed chemical kinetics and particle dynamics are to be employed for the simulation of these flames, as the decreased dimensionality of counterflow flames 
significantly reduces the computational cost. To give an example, a recent computational work [110] on an ethylene/air coflow flame using a semi-detailed chemical reaction mechanism $[111,112]$ and a sectional soot model was reported to take a wall time of more than 150 hours on a 400 CPU Linux cluster (i.e., 60,000 CPU hours) for convergence; similar work for one-dimensional counterflow flames typically takes no more than several hours on a PC. Also, it is generally believed that a reacting zone of premixed nature is responsible for the stabilization and attachment of the flame to the nozzle in a coflow diffusion flame. This premixed zone exists due to the inter-diffusion of fuel and oxidizer in the vicinity of the burner nozzle, where chemical reactions are inhibited by heat and radical losses to the wall $[109,113]$. Therefore, coflow diffusion flames are also affected by the characteristics of this stabilizing premixed edge flame. For this reason, some fundamental aspects of diffusion combustion are thought not to be well represented by coflow diffusion flames, while counterflow diffusion flames are recognized as pure diffusion flames [109]. Finally, the residence time in coflow flames is relatively long and sometimes difficult to parameterize. In counterflow flames, residence time and stretch rate can be adjusted by varying fuel and/or oxidizer flow velocities, providing a unique way to study soot chemistry with variable residence time. Also, its much shorter residence time is more representative of the turbulent processes in practical flames [114]. Despite the above merits, it is important to point out that counterflow flames also have disadvantages. For instance, a counterflow flame is usually rather confined in space, necessitating high spatial resolution for its diagnostic. In contrast, coflow flames are more spread out spatially which makes probe sampling of the gas phase or particulates much easier.

Major differences exist in the sooting characteristics between coflow and counterflow diffusion flames. Soot formed in the fuel-rich region of a normal coflow flame is always convected downstream, towards the high temperature flame front where fuel and oxidizer are mixed stoichiometrically. As a result, oxidation of soot by oxygen and hydroxyl radical is inevitable. In fact, the widely referred smoke point condition is a critical condition where soot formation is balanced just by its oxidation 
[104, 115-117]. But for counterflow diffusion flames, the absence or presence of soot oxidation depends on the relative position between the stagnation plane and the flame front. By adjusting the dilution ratio of the fuel and oxidizer streams (and thus the stoichiometric mixture fraction), it is possible to establish a soot formation (SF) flame where soot particles, once formed, are convected away from the flame without further oxidation [73]. In this way, the soot formation process, as well as the physicochemical features of the flame-generated soot, can be investigated with little interference from soot oxidation. Detailed descriptions of the sooting processes in diffusion flames are covered in a later section, but it can be seen from the above discussion that counterflow flames provide a well-defined, canonical configuration to complement coflow diffusion flames for soot research on diffusion flames.

The literature on soot formation is extensive and excellent reviews [18, 31, 34, 99, 107, 118-128] were periodically published to summarize research progress. Many of these reviews had a specific focus. For example, in addition to providing an excellent theoretical discussion on the general kinetic pathways of soot formation, experimental data reviewed by Wang [18] were largely based on studies of burner-stabilized premixed flames. The work of Eremin [31] focused on the formation of soot particles in shock tubes while Tree and Svesson [124] analyzed soot processes in compression ignition engines. D'Anna [125] insightfully examined both numerical and experimental aspects of nanoparticle formation in laminar flames, focusing on premixed and coflow diffusion flames, while with limited coverage on counterflow diffusion flames. Karataş and Gülder [107] offered a dedicated review on high-pressure coflow diffusion flames. Despite the importance of soot studies in laminar counterflow flames, and the many important conclusions drawn from them, there has been no dedicated review in the open literature summarizing soot research in these flames, which dates back to 1980 s and continues to contribute to our expanding knowledge on soot. The special features of soot processes in counterflow flames certainly deserves such efforts. Motivated by this, we intend to provide a comprehensive survey of research outcomes resulting from investigations of soot formation 
in counterflow flames, complementing existing reviews on soot research in many other flame/reactor configurations.

\subsection{Structure of this review}

Following is the structure of this survey of soot research in laminar counterflow flames. First, an overview of the soot formation pathways is revisited, with special attention paid to the most recent progress. The discussion follows the generally acknowledged major processes of soot formation [122]: 1) gas-phase precursor formation; 2) soot nucleation; 3) particle surface growth, particle coalescence and agglomeration; and 4) soot oxidation and fragmentation. Experimental methods for soot diagnostics are then briefly discussed to provide necessary background for following discussions on the general sooting structures of diffusion and partially-premixed flames. Similarities and differences in the soot evolution processes between coflow and counterflow flames are also highlighted. Experimental and numerical counterflow flame-based soot studies are then extensively reviewed and the materials are organized based on the various factors affecting soot formation, including fuel molecular structure, fuel additives, pressure, temperature, residence time, external fields, and flame unsteadiness. The discussion on fuel molecular structure also summarizes various quantitative indices developed to represent sooting tendencies of different fuels, which may find application in assessing and formulating surrogate fuels for sooting tendencies. These indices include the conventional smoke point, the coflow flame-based yield soot index and the newly proposed, CDF-based sooting temperature index and sooting sensitivity index. This review concludes with a brief summary and an outlook on research needs, with respect to both experimental and numerical aspects, for promoting fundamental understanding of soot formation in counterflow flames. 


\section{Pathways of soot formation}

The physicochemical pathways from gas-phase fuels to soot particles in flames are not only complex but intellectually intriguing. It may seem impossible that structurally ordered solids can be formed under violent entropy-increasing flame conditions. In fact, as noted by Wang [18], dehydrogenation from hydrocarbon fuel molecules and the accompanied release of $\mathrm{H}_{2}$ are important in decreasing the Gibbs free energy of the system, driving soot formation processes forward. Nevertheless, such a thermodynamic driving force is moderate, so the soot process is kinetically controlled and can be highly reversible [18].

Although many details are still not fully understood, decades of extensive research have led to the general belief that soot formation starts with the formation of gas-phase molecular species from fuel pyrolysis, followed by incipient ring formation, PAH growth, particle nucleation, heterogeneous surface reaction, and particle-particle interactions. For clarification, Figure 1 shows a conceptual schematic of this overall physicochemical pathway. This now well-known pathway is a frequent review subject, although new findings are added continuously. This section provides a systematic summary of the most recent discoveries in soot formation mechanisms. The material in this section will not be restricted to counterflow flames, since the fundamental chemistry involved in the process of soot formation is expected to share similarities among different flame configurations [107].

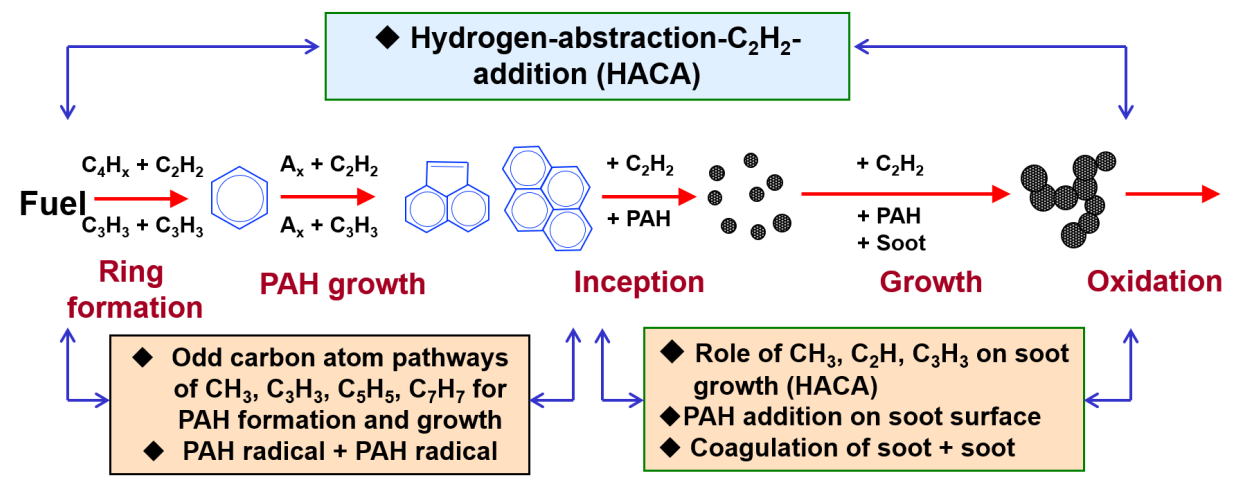

Figure 1. Schematic of overall pathways for soot formation. 
It is our intention to provide a detailed description of our up-to-date understanding on soot formation. We think that such information can be a useful background knowledge to put the results of the to-be-reviewed counterflow-based fundamental soot studies into the context of understanding soot formation from first principles. Before detailed presentation, it is worthwhile to point out that the discussion below may contain historical developments that overlapped with previous reviews such as Richter and Howard [121], McEnally et al [123], D'Anna [125], Wang [18], and Karatas and Gülder [107]. Although we focused on the most recent developments after 2010, a certain degree of overlaps is unavoidable for a systematic discussion.

\subsection{Gas phase precursor formation}

Although polyacetylenes [129, 130] and ionic species [131] have historically been proposed as soot precursors, it is now widely accepted that the formation of PAHs is the key step in bridging the gap between gaseous fuel molecules and solid soot particles. Detailed reaction pathways are still needed to validate polyacetylenes hypothesis against measurements. The experimental results of low concentrations of chem-ions [132] and low levels of ionization for large hydrocarbons in fuel rich sooting flames [133], as well as the absence of ionic species in soot-forming pyrolysis environments [134], support evidence against the ionic mechanism. These are in contrast to the fact that the PAH hypothesis has been substantiated by numerous experimental and numerical studies [122]. For example, through a computational investigation, Stein and Fahr [135] found that the most thermodynamically stable species at high temperatures in a $\mathrm{H}_{2} / \mathrm{C}_{2} \mathrm{H}_{2}$ mixture were PAH molecules, suggesting their important roles in soot formation. The more recent work by Michelson, Violi and coworkers [136-138] suggesting the importance of non-stabilomer PAHs in soot formation will be discussed later. In addition, on a macroscopic scale, it was observed in a series of shock tube pyrolysis experiments that aromatic fuels (benzene and toluene) and cyclic compounds that can readily aromatize (e.g., 1,4-cyclohexadiene) have the highest soot yields, followed in order by species that can also have ring ruptures competing with aromatization (e.g., cyclopentadiene) and those that can 
be easily fragmented (e.g., cyclohexane) [139]. Many recent PAH-based soot models with detailed chemical kinetics have gained unprecedented success in predicting the sooting characteristics of hydrocarbon flames, especially in terms of the effects of fuel molecular structures [112, 140]. Nevertheless, we are probably still not in a position to exclude the possible contribution of polyacetylenes and ionic species to soot formation, and more research is required to unravel this problem. In this study, however, we will presume that PAHs are the primary soot precursors and detailed discussions on other alternatives are beyond the scope of this review.

\subsubsection{Formation of first aromatic ring}

A number of reaction routes have been proposed for the growth of PAHs in hydrocarbon flames, and the relative importance of each individual pathway was shown to be notably dependent on fuel molecular structure, as well as pressure and temperature conditions [121]. For aliphatic fuels, it may be logical to expect the growth of PAHs to start from the first aromatic ring, which in many cases is benzene or phenol radical. Pioneering works on pyrolysis/oxidation of acetylene $\left(\mathrm{C}_{2} \mathrm{H}_{2}\right)$ [141-143], $\mathrm{C}_{2} \mathrm{H}_{2} / \mathrm{H}_{2}$ mixture [144], methyl chloride $\left(\mathrm{CH}_{3} \mathrm{Cl}\right)$ [145], and rich premixed flames of $\mathrm{C}_{2} \mathrm{H}_{2}$ [146], ethylene $\left(\mathrm{C}_{2} \mathrm{H}_{4}\right)$ [147], and 1,3-butadiene $\left(1,3-\mathrm{C}_{4} \mathrm{H}_{6}\right)$ [148] suggest that the following reactions are important for benzene (A1) formation from small aliphatics:

$\mathrm{n}-\mathrm{C}_{4} \mathrm{H}_{3}+\mathrm{C}_{2} \mathrm{H}_{2} \rightarrow \mathrm{A} 1-\quad \mathrm{R} 1$

$\mathrm{n}-\mathrm{C}_{4} \mathrm{H}_{5}+\mathrm{C}_{2} \mathrm{H}_{2} \rightarrow \mathrm{A} 1+\mathrm{H}$

where $\mathrm{C}_{2} \mathrm{H}_{2}$ is acetylene, $\mathrm{n}-\mathrm{C}_{4} \mathrm{H}_{3}$ has the structure of $\mathrm{HC} \equiv \mathrm{C}-\mathrm{CH}=\dot{\mathrm{C}} \mathrm{H}, \mathrm{n}-\mathrm{C}_{4} \mathrm{H}_{5}$ has $\mathrm{H}_{2} \mathrm{C}=\mathrm{CH}-\mathrm{CH}=\dot{\mathrm{C}} \mathrm{H}$, A1- and A1 represent phenyl radical and benzene, respectively. In addition, it was shown that the addition of vinyl radical $\left(\mathrm{C}_{2} \mathrm{H}_{3}\right)$ to $\mathrm{C}_{2} \mathrm{H}_{2}$ was pivotal in the production of $\mathrm{n}-\mathrm{C}_{4} \mathrm{H}_{5}$ in flames of aliphatic fuels [148] (except for 1,3- $\mathrm{C}_{4} \mathrm{H}_{6}$ flame where $\mathrm{H}$ abstraction from the fuel molecule can directly lead to $\left.\mathrm{n}-\mathrm{C}_{4} \mathrm{H}_{5}\right)$ and ethynyl radical $\left(\mathrm{C}_{2} \mathrm{H}\right)$ addition to $\mathrm{C}_{2} \mathrm{H}_{2}$ was important for $\mathrm{n}-\mathrm{C}_{4} \mathrm{H}_{3}$ formation $[143,146]$. 
The importance of R1 and R2 was substantiated by Westmoreland and coworkers [149, 150], who screened various benzene formation pathways by comparing experimentally-obtained species mole fraction profiles with predicted ones in a series of low-pressure fuel-rich premixed $\mathrm{C}_{2} \mathrm{H}_{2} / \mathrm{O}_{2} / \mathrm{Ar}$ flames. They employed the concept of chemical activation and considered the effects of pressure falloff, proposing rate constants of relevant reactions at different pressures instead of using high-pressure limits $[149,151]$. Although the recombination of two propargyl radicals $\left(\mathrm{C}_{3} \mathrm{H}_{3}\right)$ was also seen to be a promising route for benzene formation in the initial screening, they ruled out this possibility as they presumed that subsequent cyclization/hydrogen redistribution of the $\mathrm{C}_{3} \mathrm{H}_{3}-\mathrm{C}_{3} \mathrm{H}_{3}$ adduct would result in serious difficulties [149].

Nevertheless, the role of $\mathrm{C}_{3} \mathrm{H}_{3}$ in cyclization reactions has attracted considerable research effort since late 1980 s. This is due partly to the recognition that $\mathrm{C}_{3} \mathrm{H}_{3}$ is one of the most important resonantly stabilized radicals (RSRs) during hydrocarbon combustion. Being an $\mathrm{RSR}, \mathrm{C}_{3} \mathrm{H}_{3}$ is more stable than ordinary radicals and its chance for survival in a hostile combustion environment is greater. As a result, it can be accumulated to a much higher concentration, making its reactions attractive cyclization routes. It is also noteworthy that although $\mathrm{C}_{3} \mathrm{H}_{3}$ is less reactive than ordinary radicals, the $\mathrm{C}_{3} \mathrm{H}_{3}-\mathrm{C}_{3} \mathrm{H}_{3}$ addition is still a radical-radical reaction, so its rates can be significantly higher than those of radical-molecule reactions like $\mathrm{R} 1$ and $\mathrm{R} 2$ [152].

Miller and Melius [152] performed a numerical study on premixed fuel-rich $\mathrm{C}_{2} \mathrm{H}_{2}$ flames and used a kinetic model in which the isomers of $\mathrm{C}_{4} \mathrm{H}_{3}$ and $\mathrm{C}_{4} \mathrm{H}_{5}$ were distinguished. The following $\mathrm{H}$-atom assisted isomeric reactions were also included:

$$
\begin{array}{ll}
\text { n- } \mathrm{C}_{4} \mathrm{H}_{3}+\mathrm{H} \rightarrow \mathrm{i}-\mathrm{C}_{4} \mathrm{H}_{3}+\mathrm{H} & \text { R3 } \\
\mathrm{n}-\mathrm{C}_{4} \mathrm{H}_{5}+\mathrm{H} \rightarrow \mathrm{i}-\mathrm{C}_{4} \mathrm{H}_{5}+\mathrm{H} & \text { R4 }
\end{array}
$$

where $\mathrm{i}-\mathrm{C}_{4} \mathrm{H}_{3}$ is resonantly stabilized between $\mathrm{H}_{2} \mathrm{C}=\mathrm{C}=\mathrm{C}=\dot{\mathrm{C}} \mathrm{H}$ and $\mathrm{H}_{2} \mathrm{C}=\dot{\mathrm{C}}-\mathrm{C} \equiv \mathrm{CH}$, and i- $\mathrm{C}_{4} \mathrm{H}_{5}$ between $\mathrm{H}_{2} \mathrm{C}=\mathrm{C}=\mathrm{CH}-\dot{\mathrm{C}} \mathrm{H}_{2}$ and $\mathrm{H}_{2} \mathrm{C}=\dot{\mathrm{C}}-\mathrm{CH}=\mathrm{CH}_{2}$. They argued that the benzene production rates from R1 and R2 alone were too slow to account for the experimentally-measured benzene 
concentrations in the $\mathrm{C}_{2} \mathrm{H}_{2}$ flame. They showed that the rapid consumption of $n-\mathrm{C}_{4} \mathrm{H}_{3}$ and $n-\mathrm{C}_{4} \mathrm{H}_{5}$ (through R3 and R4, respectively), which were not included in earlier models, could explain the insignificance of R1 and R2 at flame temperatures. Instead, they [152] proposed that the following reactions are the leading route for benzene formation in $\mathrm{C}_{2} \mathrm{H}_{2}$ flames:

$$
\begin{array}{ll}
\mathrm{C}_{3} \mathrm{H}_{3}+\mathrm{C}_{3} \mathrm{H}_{3} \rightarrow \mathrm{A} 1-+\mathrm{H} & \mathrm{R} 5 \\
\mathrm{C}_{3} \mathrm{H}_{3}+\mathrm{C}_{3} \mathrm{H}_{3} \rightarrow \mathrm{A} 1 & \mathrm{R} 6
\end{array}
$$

The dominant contribution of R5 and R6 to benzene formation was supported by Stein et al. [153], who independently modeled benzene formation rates in $\mathrm{C}_{2} \mathrm{H}_{2}$ flames. It has also been substantiated by the following works on flames of other aliphatic fuels such as methane $\left(\mathrm{CH}_{4}\right)$ [154], $\mathrm{C}_{2} \mathrm{H}_{4}[155$, 156], ethane $\left(\mathrm{C}_{2} \mathrm{H}_{6}\right)$ [154], propene $\left(\mathrm{C}_{3} \mathrm{H}_{6}\right)$ [157-159], propane $\left(\mathrm{C}_{3} \mathrm{H}_{8}\right)$ [160], n-butane $\left(\mathrm{n}-\mathrm{C}_{4} \mathrm{H}_{10}\right)$ [161], 1-pentene $\left(1-\mathrm{C}_{5} \mathrm{H}_{10}\right)$ [162], and 1,3-pentadiene $\left(1,3-\mathrm{C}_{5} \mathrm{H}_{8}\right)$ [163]. In addition, the propargyl recombination route was also necessary to explain the relative tendency of benzene formation during the oxidation of $\mathrm{C}_{2} \mathrm{H}_{2}$, allene $\left(\mathrm{C}_{3} \mathrm{H}_{4}-\mathrm{A}\right)$, and propyne $\left(\mathrm{C}_{3} \mathrm{H}_{4}-\mathrm{P}\right)$ in a jet stirred reactor [164]. In particular, experimentally-observed synergistic effects [165] on the formation of PAHs and soot in CDFs of ethylene-propane binary mixture fuels provided further evidence on the importance of $\mathrm{C}_{3} \mathrm{H}_{3}$ in aromatic species formation.

The chemical pathway for propargyl recombination is in fact more complex than it may seem in R5 and R6. Based on computed potential energy surfaces, Miller and Klippenstein [166] found that three varieties of energized linear $\mathrm{C}_{6}$ adduct can be formed, and each can further isomerize among various forms, dissociate into bimolecular products or back into the reactants. Additional product channels (R7 and R8) for propargyl recombination were proposed [167] based on this theoretical analysis, and related rate coefficients for $\mathrm{R} 5$ through $\mathrm{R} 8$ were modified to reflect more recent rate calculations [168].
$\mathrm{C}_{3} \mathrm{H}_{3}+\mathrm{C}_{3} \mathrm{H}_{3} \rightarrow$ fulvene
R7
$\mathrm{C}_{3} \mathrm{H}_{3}+\mathrm{C}_{3} \mathrm{H}_{3} \rightarrow \mathrm{C}_{4} \mathrm{H}_{5} \mathrm{C}_{2} \mathrm{H}$
R8 
Here, $\mathrm{C}_{4} \mathrm{H}_{5} \mathrm{C}_{2} \mathrm{H}$ is 2-ethylnyl-1,3-butadiene and fulvene $\left(\mathrm{c}-\mathrm{C}_{5} \mathrm{H}_{4} \mathrm{CH}_{2}\right)$ can easily be converted to benzene through $\mathrm{H}$-atom-assisted isomerization [169, 170]. Of course, as pointed out by Miller and Klippenstein [166], R5 through $\mathrm{R} 8$ do not represent a complete description of $\mathrm{C}_{3} \mathrm{H}_{3}$ recombination chemistry. Nevertheless, as a lumped model, they are suitable for applications in typical flame conditions. Caution is required if they are used in low-temperature and high-pressure conditions.

Allyl radical $\left(\mathrm{C}_{3} \mathrm{H}_{5}-\mathrm{A}\right)$ is also an $\mathrm{RSR}$ and its combination with $\mathrm{C}_{3} \mathrm{H}_{3}$ was proposed as a potentially important cyclization step [160], especially under conditions where $\mathrm{C}_{3} \mathrm{H}_{5}$-A can be produced in large quantities such as in flames fueled by allene $\left(\mathrm{H}_{2} \mathrm{C}=\mathrm{C}=\mathrm{CH}_{2}\right)$ [171]. The implementation of this pathway in chemical mechanisms typically takes the following form [160]:

$\mathrm{C}_{3} \mathrm{H}_{3}+\mathrm{C}_{3} \mathrm{H}_{5}-\mathrm{A} \rightarrow$ fulvene $+\mathrm{H}+\mathrm{H} \quad$ R9

Recent developments and applications of synchrotron-based tunable VUV-ionization molecularbeam mass spectrometry, which could provide isomer-resolved intermediate species identification and quantification, have contributed significantly to our understanding on flame chemistry [172]. A number of premixed fuel-rich flames burning aliphatic fuels were investigated with this technique, and it was found that reactions involving propargyl radical (i.e., R5 through R9) are always important, while contributions from other pathways are somewhat fuel dependent $[172,173]$. For instance, the reaction between $\mathrm{i}-\mathrm{C}_{4} \mathrm{H}_{5}$ and $\mathrm{C}_{2} \mathrm{H}_{2}(\mathrm{R} 10)$ is identified as a major benzene formation pathway (through fulvene) in both premixed [167] and diffusion [174] 1,3-butadiene flames, with comparable importance to propargyl recombination.

$\mathrm{i}-\mathrm{C}_{4} \mathrm{H}_{5}+\mathrm{C}_{2} \mathrm{H}_{2} \rightarrow$ fulvene $+\mathrm{H} \quad \mathrm{R} 10$

Note that $\mathrm{i}-\mathrm{C}_{4} \mathrm{H}_{5}$ can be directly formed from the dehydrogenation of the fuel molecule, and thus can be accumulated in large concentrations in 1,3-butadiene flames.

In methyl-cyclohexane $\left(\mathrm{c}-\mathrm{C}_{6} \mathrm{H}_{11} \mathrm{CH}_{3}\right)$ flames/pyrolysis, stepwise dehydrogenation toward fulvene and benzene was seen to be a cyclization step of significant importance $[175,176]$. This is also true for cyclohexane $\left(\mathrm{c}-\mathrm{C}_{6} \mathrm{H}_{12}\right)$ flames [177-180], where stepwise dehydrogenation leads directly to 
benzene. Note that RSRs such as cyclohexenyl $\left(\mathrm{c}-\mathrm{C}_{6} \mathrm{H}_{9}\right)$ and cyclohexadienyl radicals $\left(\mathrm{c}-\mathrm{C}_{6} \mathrm{H}_{7}\right)$ are also involved in the process of cyclohexane dehydrogenation. It is also noteworthy that R2 was shown to account for $6 \%$ of the total benzene formation in a fuel-rich cyclohexane flame; the explanation was based on specific fuel destruction pathways that can generate relatively large concentrations of n- $\mathrm{C}_{4} \mathrm{H}_{5}[177]$. Besides, $\mathrm{R} 2$ was seen to contribute to $3.5 \%$ of benzene in a fuel-rich 1 -hexene $\left(1-\mathrm{C}_{6} \mathrm{H}_{12}\right)$ flame [181].

Other benzene formation pathways include the addition of $\mathrm{C}_{5}$ and $\mathrm{C}_{1}$ species, notably the reaction between cyclopentadienyl $\left(\mathrm{c}-\mathrm{C}_{5} \mathrm{H}_{5}\right)$ and methyl radical $\left(\mathrm{CH}_{3}\right)$ to form benzene through fulvene [170, 182]:

c- $\mathrm{C}_{5} \mathrm{H}_{5}+\mathrm{CH}_{3} \rightarrow \mathrm{C}_{5} \mathrm{H}_{4} \mathrm{CH}_{3}+\mathrm{H} \quad \mathrm{R} 11$

Further hydrogen loss from $\mathrm{C}_{5} \mathrm{H}_{4} \mathrm{CH}_{3}$ leads to fulvene. $\mathrm{R} 11$ has been tested in cyclopentene $\left(\mathrm{c}-\mathrm{C}_{5} \mathrm{H}_{8}\right)$ flames where $\mathrm{c}-\mathrm{C}_{5} \mathrm{H}_{5}$ can easily be formed by $\mathrm{H}$ abstraction from fuel molecules [183]. Nevertheless, the results showed that major benzene formation pathways are still propargyl recombination and R11 only plays a minor role. Miller et al. [184] explained that at flame conditions, it is typical for $\mathrm{c}_{-} \mathrm{C}_{5} \mathrm{H}_{5}$ to decompose back to $\mathrm{C}_{2} \mathrm{H}_{2}$ and $\mathrm{C}_{3} \mathrm{H}_{3}$ (R12) and as a result, $\mathrm{R} 5$ through $\mathrm{R} 8$ become dominant aromatic ring formation routes. Interestingly, however, R11 was seen to be the dominant channel for benzene formation in a recent pyrolysis study of ethylcyclohexane $\left(\mathrm{c}-\mathrm{C}_{6} \mathrm{H}_{11} \mathrm{C}_{2} \mathrm{H}_{5}\right)$ [185].

c- $\mathrm{C}_{5} \mathrm{H}_{5} \rightarrow \mathrm{C}_{3} \mathrm{H}_{3}+\mathrm{C}_{2} \mathrm{H}_{2} \quad \mathrm{R} 12$

The following reactions between ethynyl-substituted propargyl radical (i.e., i- $\mathrm{C}_{5} \mathrm{H}_{3}, \mathrm{HC} \equiv \mathrm{C}-\mathrm{C} \equiv$ $\mathrm{C}-\dot{\mathrm{C}} \mathrm{H}_{2}$, also an RSR) and $\mathrm{CH}_{3}$ were considered potential contributors to benzene formation:

$\mathrm{i}-\mathrm{C}_{5} \mathrm{H}_{3}+\mathrm{CH}_{3} \rightarrow$ fulvene $\quad \mathrm{R} 13$

$\mathrm{i}-\mathrm{C}_{5} \mathrm{H}_{3}+\mathrm{CH}_{3} \rightarrow \mathrm{A} 1-+\mathrm{H} \quad \mathrm{R} 14$

$\mathrm{i}-\mathrm{C}_{5} \mathrm{H}_{3}+\mathrm{CH}_{3} \rightarrow \mathrm{A} 1$

Although the presence of $\mathrm{i}-\mathrm{C}_{5} \mathrm{H}_{3}$ has been experimentally confirmed in fuel-rich flames [186], direct evidence of the importance of R13 through R15 still awaits. In fact, based on a comparison with the propargyl recombination reactions, Miller et al. [184] argued the rates of R13 through R15 should be 
very small. Also, the formation of benzene through linear $\mathrm{C}_{6} \mathrm{H}_{\mathrm{x}}$ molecules such as 1,3-hexadien-5yne $\left(\mathrm{HC} \equiv \mathrm{CCH}=\mathrm{CHCH}=\mathrm{CH}_{2}\right)$ and $1,3,5$-hexatriene $\left(\mathrm{CH}_{2}=\mathrm{CHCH}=\mathrm{CHCH}=\mathrm{CH}_{2}\right)$ were suggested to be important for premixed $\mathrm{C}_{2} \mathrm{H}_{4}[187]$ and $\mathrm{C}_{2} \mathrm{H}_{2}$ [146] flames.

From the above discussions, it is clear that — with the notable exception of R1 and R2 - the majority of the important benzene formation pathways identified thus far for flames of aliphatic fuels involve RSRs, suggesting their significance.

It is useful to point out that benzene is not necessarily the first aromatic ring to form during the combustion of aliphatic fuels. There are several other aromatic species that can serve as precursors to grow to larger PAHs and soot. Bockhorn et al. [188] proposed the formation of phenylacetylene (c$\left.\mathrm{C}_{6} \mathrm{H}_{5} \mathrm{C}_{2} \mathrm{H}\right)$ through consecutive addition of ethynyl radical $\left(\mathrm{C}_{2} \mathrm{H}\right)$ and acetylene to diacetylene $\left(\mathrm{C}_{4} \mathrm{H}_{2}\right)$, followed by ring closure, thus bypassing benzene. Other notable examples include the ring enlargement reaction between cyclopentadienyl radical and $\mathrm{C}_{2} \mathrm{H}_{2}$ to form cycloheptatrienyl radical $\left(\mathrm{c}-\mathrm{C}_{7} \mathrm{H}_{8} \cdot\right)$, which can then isomerize to benzyl radical $\left(\mathrm{A}_{\left.1 \mathrm{CH}_{2} \cdot\right)}\right)$ [189]. This pathway R16, as shown below, was found to be important in cyclopentene flames [190].

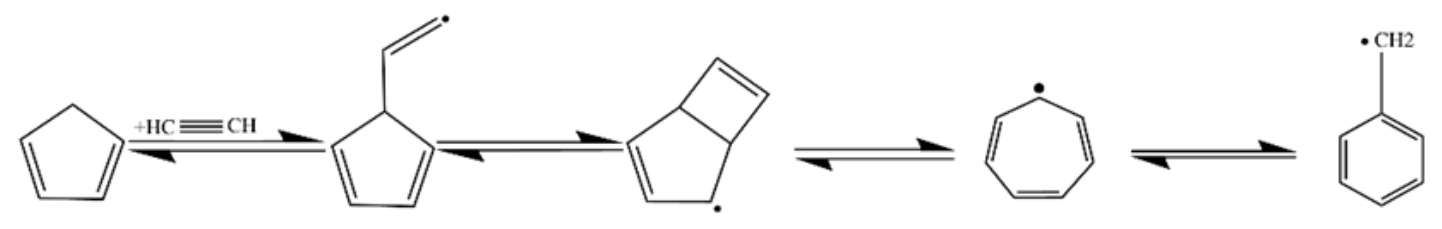

The recombination of cyclopentadienyl radical has been proposed as a formation pathway toward two-ring aromatics (i.e., naphthalene, $\mathrm{A} 2, \mathrm{C}_{10} \mathrm{H}_{8}$ ) $[170,191]$. Melius et al. [170] in particular suggested the following reaction paths, $\mathrm{R} 17$ and $\mathrm{R} 18$, through $9-\mathrm{H}-\mathrm{fulvalenyl}$ radical $\left(\mathrm{c}-\mathrm{C}_{5} \mathrm{H}_{5}-\mathrm{c}-\right.$ $\left.\mathrm{C}_{5} \mathrm{H}_{4}\right)$ :

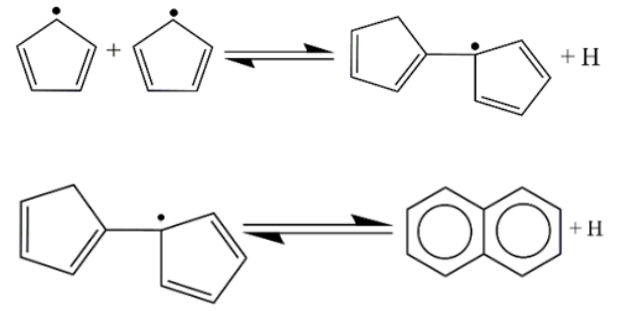


$\mathrm{c}-\mathrm{C}_{5} \mathrm{H}_{5}+\mathrm{c}-\mathrm{C}_{5} \mathrm{H}_{5} \rightarrow \mathrm{A} 2+2 \mathrm{H}$

Marinov et al. [161] provided rate constants for the overall reaction R19 which were included in many kinetic models for flame simulations $[192,193]$. Several other studies used the reduced preexponential factor for R19 [194]. Recent investigations [195] found that agreement between experimental data and those predicted could be obtained by using a rate constant one order of magnitude slower than that suggested by Marinov et al. In addition, Mebel and Kislov [196] argued that the primary product of 9-H-fulvalenyl decomposition should be fulvalene (i.e., bicyclopentadienylidene, $\left.\mathrm{c}-\mathrm{C}_{5} \mathrm{H}_{4}=\mathrm{c}-\mathrm{C}_{5} \mathrm{H}_{4}\right)$ instead of naphthalene. This motivated further theoretical study on $\mathrm{c}-\mathrm{C}_{5} \mathrm{H}_{5}$ recombination reactions and, through a revisit of the $\mathrm{C}_{10} \mathrm{H}_{10}$ potential energy surface, Cavallotti and Polino [197] proposed an alternative reaction channel through $\mathrm{H}$-azulyl radical $\left(\mathrm{C}_{10} \mathrm{H}_{9}\right)$, which is known to decompose easily to $\mathrm{A} 2$ and $\mathrm{H}$ atom.

Reactions involving propargyl and/or radical-substituted propargyl can also lead to the formation of aromatic species bypassing benzene. Miller et al. [184] referred the following reactions, involving methyl substituted propargyl (1-methylallenyl, $\mathrm{H}_{3} \mathrm{C}-\mathrm{C} \equiv \mathrm{C}-\dot{\mathrm{C}} \mathrm{H}_{2}$ ), to be the most important among this class:

$\mathrm{C}_{3} \mathrm{H}_{3}+\mathrm{CH}_{3} \mathrm{CCCH}_{2} \rightarrow \mathrm{A} 1 \mathrm{CH}_{2}+\mathrm{H} \quad \mathrm{R} 20$

$\mathrm{C}_{3} \mathrm{H}_{3}+\mathrm{CH}_{3} \mathrm{CCCH}_{2} \rightarrow \mathrm{A}_{1} \mathrm{CH}_{3} \quad \mathrm{R} 21$

$\mathrm{CH}_{3} \mathrm{CCCH}_{2}+\mathrm{CH}_{3} \mathrm{CCCH}_{2} \rightarrow$ o-xylene $\quad$ R22

where $\mathrm{A}_{1 \mathrm{CH}}$ and $\mathrm{A}_{1 C H}$ represent benzyl radical and toluene, respectively and o-xylene is 1,2xylene.

For ease of future reference, Figure 2 summarizes these important formation pathways of the first aromatic ring in flames of aliphatic fuels. 


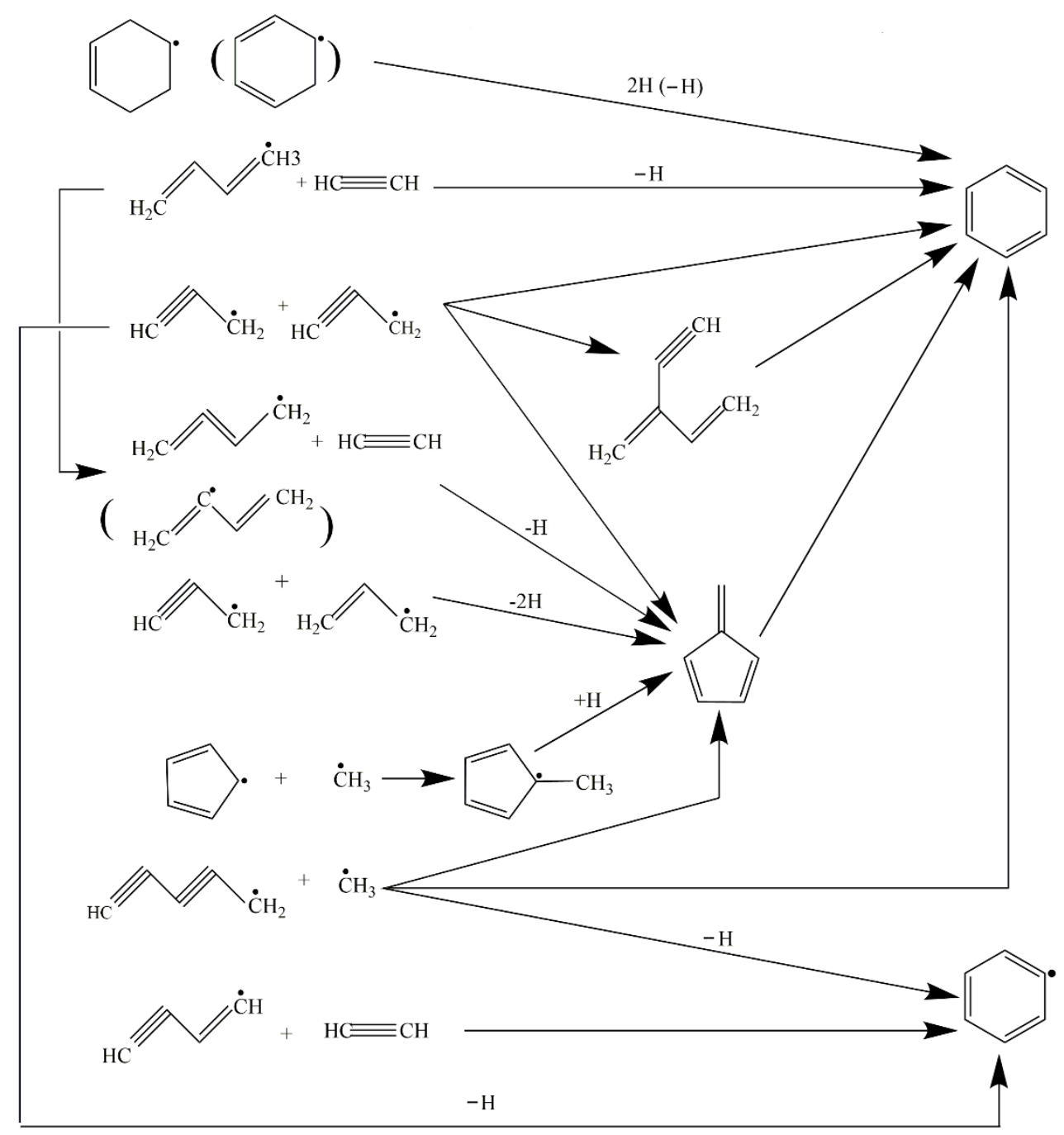

Figure 2. Major formation pathways of the first aromatic ring in flames of aliphatic fuels.

\subsubsection{Molecular growth beyond the first aromatic ring}

The necessity to form the first aromatic ring for the initiation of soot formation applies only to aliphatic fuels. For combustion systems fueled by aromatics, molecular growths beyond the first aromatic ring become the critical and rate-limiting steps toward soot formation. In such cases, the aromatic rings must be broken to provide the intermediate species required for subsequent molecular growth, which will lead to PAHs with increasingly complicated structures. It may not be difficult to imagine that the number of reaction pathways involved in forming these large PAH molecules grows exponentially with their sizes. Wang [18] pointed out that it is still not known whether the number can be treated as finite. On the other hand, it becomes complicated to obtain direct experimental 
evidence of any proposed reaction pathways for large PAHs, caused primarily for the following reasons. First, the concentrations of large PAHs in flames are typically very low. In fact, it was reported that peak mole fractions of PAHs can drop by one order of magnitude with every growth of two fused rings [18]. Besides the slow kinetics for PAH growth, this can also be understood from the fact that as PAHs get larger, they are more likely to form soot, removing themselves from the gas phase. The result is that instruments with continuous improvements in detection limits are needed if increasingly larger species are to be measured. Second, many isomers exist for large PAHs, and isomeric-specific identification becomes progressively more difficult as PAHs grow larger. For example, as a major isomer-specific measurement technique for small molecules, the synchrotronbased photo-ionization flame-sampling molecular beam mass spectrometry (MBMS) is confronted with significant challenges in isolating isomers of large molecules due (partially) to the uncertainty of obtaining their ionization energies. Nevertheless, theoretical and experimental investigations during the past decades did provide considerable knowledge about the formation pathways of large PAHs, which is being used successfully in a variety of models for the simulation of soot.

The most well-known PAH growth pathway is the hydrogen-abstraction- $\mathrm{C}_{2} \mathrm{H}_{2}$-addition mechanism (or HACA for short) proposed by Frenklach, Wang and coworkers [141, 142, 146, 198]. In its essence, this HACA mechanism represents a repetitive reaction sequence of 1) radical activation by $\mathrm{H}$-atom abstraction from stable molecules and 2) $\mathrm{C}_{2} \mathrm{H}_{2}$ molecule addition to the radical site. Taking the growth from benzene (A1) to naphthalene (A2) as an example, the HACA mechanism is realized as:

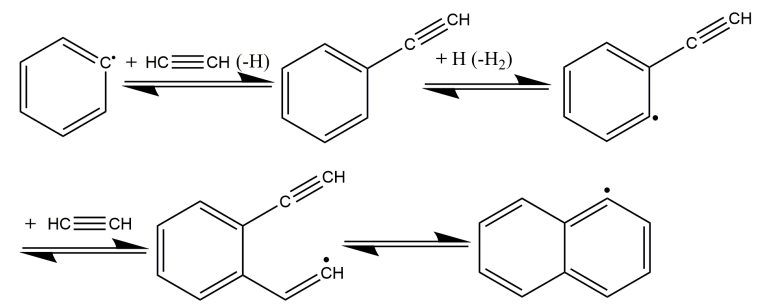

Alternatively, the addition of acetylene to phenyl radical can also lead to naphthalene formation through the following reaction: 


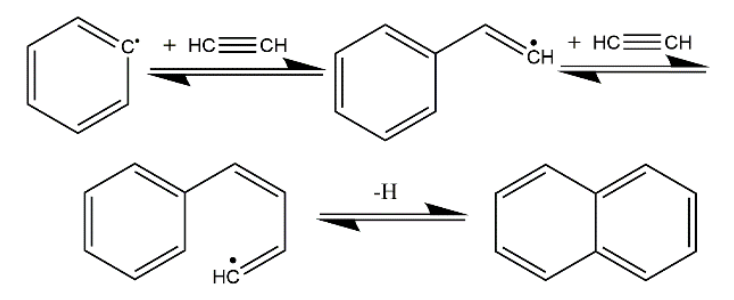

R23 and R24 were initially included in the model for the prediction of soot formation in shock tube pyrolysis of $\mathrm{C}_{2} \mathrm{H}_{2}$ [141], and it was found that $\mathrm{R} 23$ played the dominant role. Frenklach et al. [141] attributed this to the instability of the $\mathrm{A} 1 \mathrm{CHCH}$ adduct, which has a greater likelihood to decompose to the stable phenylacetylene molecule before further $\mathrm{C}_{2} \mathrm{H}_{2}$ addition can occur. After the formation of naphthalene, further hydrogen abstraction from the naphthalene molecule (by hydrogen atom) and subsequent $\mathrm{C}_{2} \mathrm{H}_{2}$ addition can lead to the formation of phenanthrene. Similar processes continue to form larger and larger PAHs, thus repetitive. Using the notations $\mathrm{A}_{i}$ and $\mathrm{A}_{i \text { - }}$ to represent aromatic species and its radical with $i$-number of rings, such a repetitive process can be represented in the following sequences:
$\mathrm{A}_{i}+\mathrm{H} \rightleftharpoons \mathrm{A}_{i^{-}}+\mathrm{H}_{2}$
R25
$\mathrm{A}_{i^{-}}+\mathrm{C}_{2} \mathrm{H}_{2} \rightleftharpoons \mathrm{A}_{i} \mathrm{C}_{2} \mathrm{H}_{2}$
R26
$\mathrm{A}_{i} \mathrm{C}_{2} \mathrm{H}_{2}+\mathrm{C}_{2} \mathrm{H}_{2} \rightleftharpoons \mathrm{A}_{i+1}$

or

$\mathrm{A}_{i^{-}}+\mathrm{C}_{2} \mathrm{H}_{2} \rightleftharpoons \mathrm{A}_{i} \mathrm{C}_{2} \mathrm{H}+\mathrm{H} \quad \mathrm{R} 28$

$\mathrm{A}_{i} \mathrm{C}_{2} \mathrm{H}+\mathrm{C}_{2} \mathrm{H}_{2} \rightleftharpoons \mathrm{A}_{(i+1)^{-}} \quad \mathrm{R} 29$

It is important to note that as the related thermodynamic driving forces are only moderate, the HACA reaction sequences are highly reversible. In fact, the degree of reversibility of R25-R29 determines whether the HACA steps can contribute to PAH growth [122]. Therefore, reliable thermodynamic data of PAH species [199], from which reverse reaction rates can be calculated, become a prerequisite for accurate modelling of PAH and soot formation.

As Wang [18] pointed out, the HACA mechanism describes a process that satisfies the core thermodynamic and kinetic requirements of a sooting process. On one hand, soot precursors must 
survive hostile flame environments, and in this regard, peri-condensed PAHs are known to be among the most important stable species in sooting flames. On the other hand, before further molecular growth can occur, stable PAHs must be activated to form corresponding aryl radicals, achievable via hydrogen abstraction reactions. As the most important intermediates in sooting flames, $\mathrm{C}_{2} \mathrm{H}_{2}$ serves as the building block for mass growth. In addition, these reactions have energy barriers and are highly reversible, giving temperature a critical role. When detailed structures of PAH and their radicals are considered, the addition of $\mathrm{C}_{2} \mathrm{H}_{2}$ can have many different and complicated realizations- $\mathrm{R} 25$ through R29 are only global representations. For more details we refer to a recent work by Frenklach and coworkers [200] in which a comprehensive list of reaction channels is provided. Despite its central importance, the HACA mechanism may be only one realization of the complex PAH growth pathways. Several other reaction sequences have also been shown to contribute to PAH growths.

Panariello et al. [201] sampled from an atmospheric fuel-rich $\mathrm{C}_{2} \mathrm{H}_{4} / \mathrm{O}_{2}$ premixed flame and performed species detection using molecular beam time-of-flight (TOF) mass spectrometry, primarily targeting high molecular weight species. Subsequent fast Fourier transform (FFT) analysis of the mass spectra indicated that, in addition to $\mathrm{C}_{2} \mathrm{H}_{2}$ addition, $\mathrm{PAH}$ growth can be achieved with the addition of methylene (- $\left.\mathrm{CH}_{2}-\right)$ into the bay region of PAHs. The relevance of odd carbon numbered PAHs with five-member rings were also inferred. In addition, through a computational investigation, Liu et al. [202] showed that the sequence of $\mathrm{C}_{2} \mathrm{H}_{2}$ addition, intramolecular hydrogen migration, $\mathrm{CH}_{2}$ addition, and cyclization with $\mathrm{H}$ elimination can result in the formation of a five-member ring, as shown below.

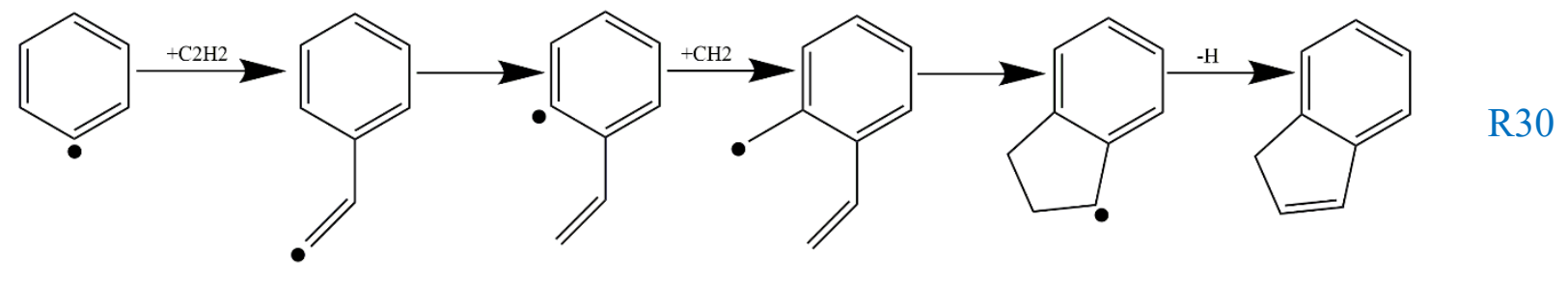


Frenklach et al. [36] performed soot modeling for 1,3-butadiene shock-tube pyrolysis and found that vinyl radicals $\left(\mathrm{C}_{2} \mathrm{H}_{3}\right)$ and vinyl acetylene $\left(\mathrm{C}_{4} \mathrm{H}_{4}\right)$ are dominant growth agents for the formation of naphthalene, through $\mathrm{R} 31$ and $\mathrm{R} 32$, respectively. Note that both $\mathrm{C}_{2} \mathrm{H}_{3}$ and $\mathrm{C}_{4} \mathrm{H}_{4}$ are pyrolysis products of the parent fuel. Their results further suggested that dominant PAH growth pathways can be fuel dependent. Similarly, Raj et al. [203] advanced the addition of $\mathrm{C}_{4} \mathrm{H}_{4}$ to pyrene radical $\left(\mathrm{C}_{16} \mathrm{H}_{9} \cdot\right)$ to form benzo(e)pyrene $\left(\mathrm{C}_{20} \mathrm{H}_{12}\right)$.<smiles>CC(C)(C)C=Cc1ccc(C=Cc2ccc(C=Cc3ccccc3)cc2)cc1</smiles><smiles>C#C/C=C/c1ccc(C(C)C)cc1/C=C/c1cccc(C#CC#CC=C)c1</smiles>

Shukla et al. [204] proposed the phenyl addition/cyclization (PAC) sequences for PAH growth based on their investigations of the pyrolysis products of toluene and toluene/benzene mixtures. Typically, PAC sequences begin with the addition of phenyl at a fusing site of an aromatic molecule. Further dehydrogenation and cyclization reactions lead to the formation of an aromatic species with more fused rings. Examples are given for the formation of fluoranthene $\left(\mathrm{C}_{16} \mathrm{H}_{10}\right)$ from naphthalene (R33) and the growth from phenanthrene to benzo(e)-pyrene (R34) [204, 205].

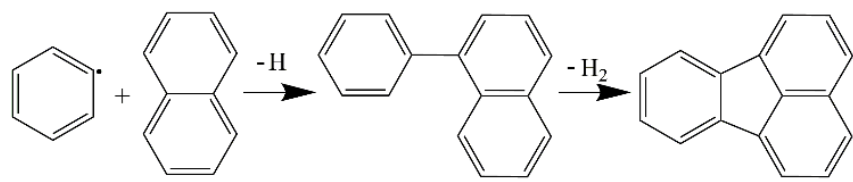

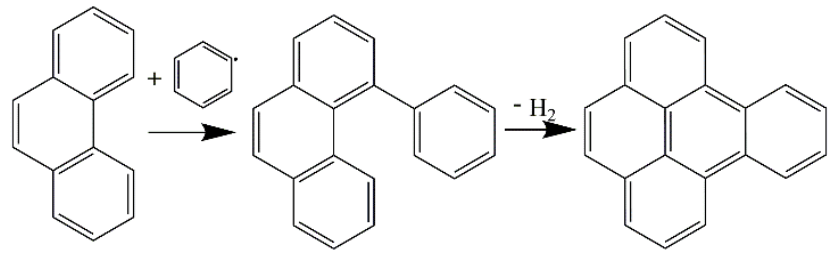


In a recently proposed PAH growth mechanism, Raj et al. [203] included two pathways for phenanthrene formation initiated by phenyl addition to phenylacetylene (R35-R36), whose possibility was suggested by the mass spectra data of Shukla et al. [204].

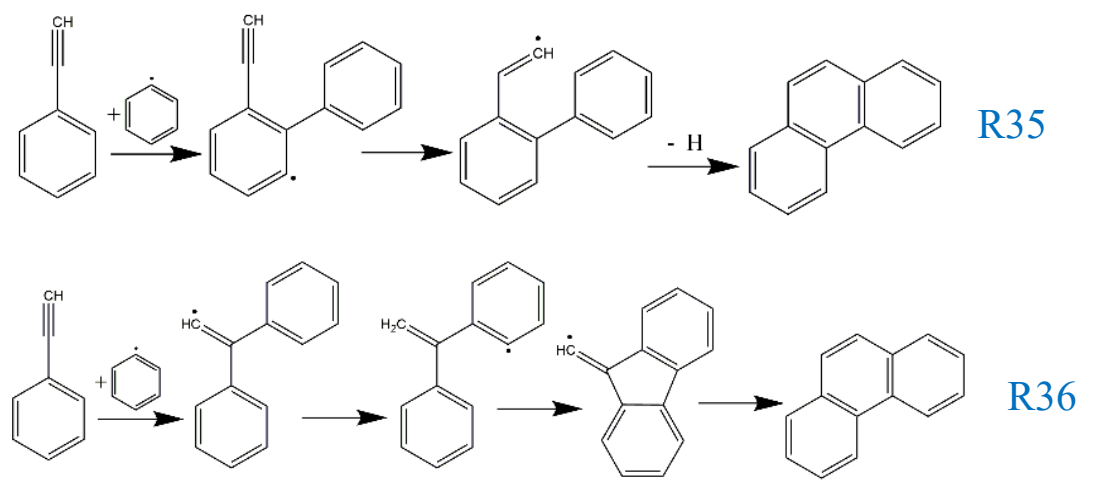

Shukla et al. [206] further examined the mass spectra of the pyrolysis products of toluene/acetone mixtures at three different temperatures and found that mass peaks occurred in bundles, with intervals of 14-mass unit. In addition, an individual major mass peak was preceded by a peak two-mass unit (sometimes also four-mass unit) lower, which became more pronounced at higher temperatures. Based on this compelling experimental evidence and relevant chemical reasoning, they proposed the methyl addition/cyclization (MAC) mechanism as a growth pathway for PAHs, which is depicted in Figure 3 [206]. Note that the MAC mechanism explains the expansion from cyclotetrafused to cyclopentafused, and then to hexagonal networks. The sequences with blue arrows in Figure 3 provide one example. Note that the MAC pathways were also substantiated by more recent mass spectral studies of counterflow diffusion flames of $\mathrm{C}_{4}$ hydrocarbons [207, 208]. 


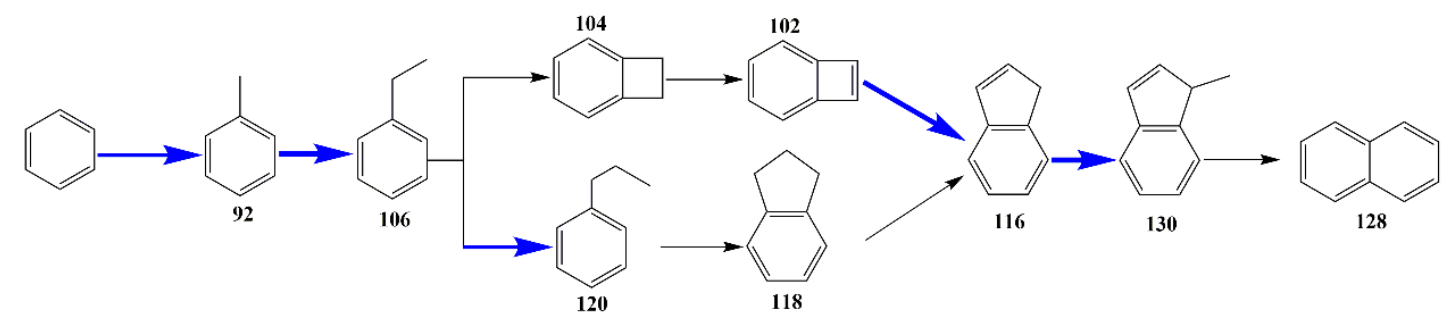

(a)
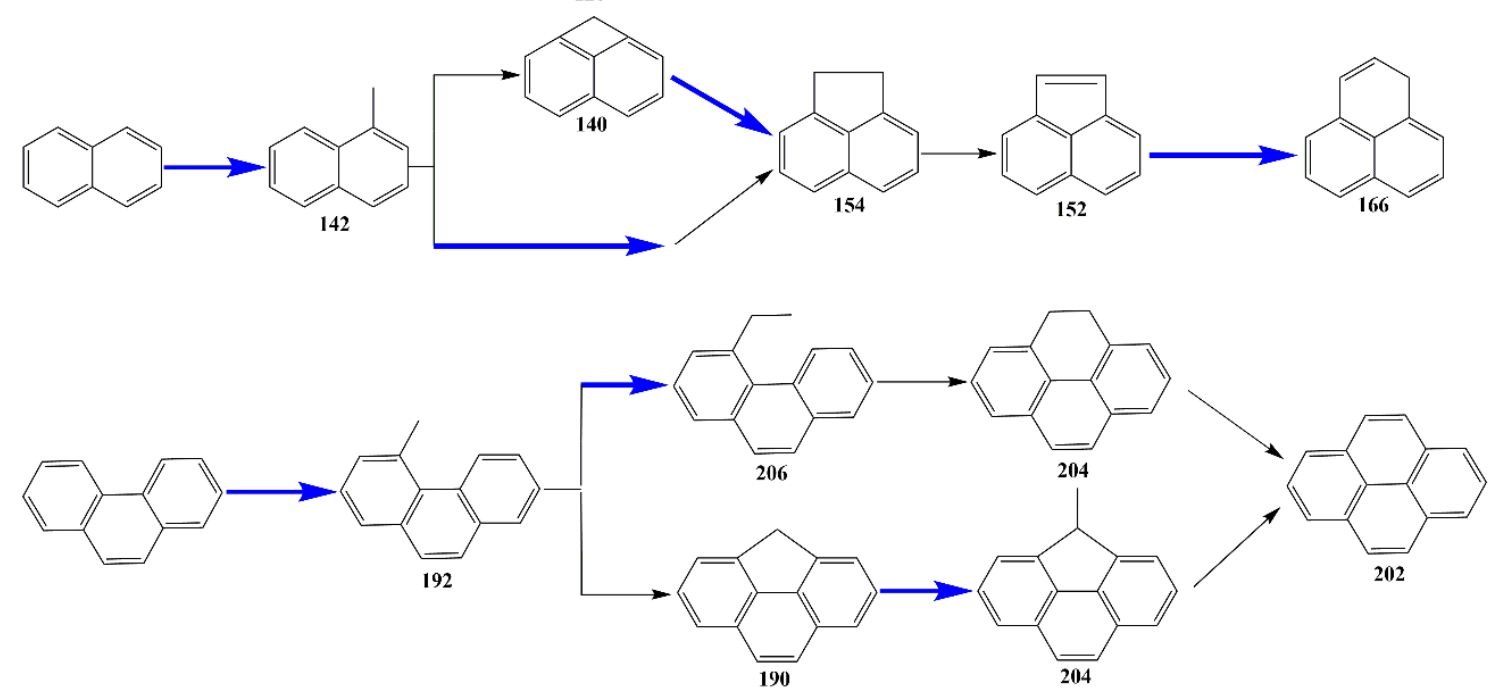

(c)

Figure 3. Methyl addition/cyclization pathways for PAH growth, shown here, are detailed pathways from benzene to naphthalene (a), naphthalene to $1 \mathrm{H}$-phenalene (b) and phenanthrene to pyrene (c). Blue arrows represent methyl addition $\mathrm{H}$-abstraction reactions. Numbers below each molecular structure are its molecular mass. Redrawn using data from Shukla et al. [206].

Following the pattern for biphenyl production through the addition of phenyl radical $\left(\mathrm{c}-\mathrm{C}_{6} \mathrm{H}_{5}\right)$ to benzene (R37) [36, 209], Böhm et al. [210] demonstrated more general combinative growth (ringring condensation) pathways which are primarily driven by biaryl reactions. It was also found that the ring-ring condensation pathway plays a dominant role in $\mathrm{PAH}$ growth during $\mathrm{C}_{2} \mathrm{H}_{2}$ and benzene pyrolysis [210].<smiles>[c]1ccccc1Cc1ccc(-c2ccccc2)cc1</smiles> 
In addition to $\mathrm{C}_{2} \mathrm{H}_{2}, \mathrm{CH}_{3}$ and phenyl radicals, the resonantly stabilized $\mathrm{C}_{3} \mathrm{H}_{3}$ radical was also suggested as an important contributor to PAH growth. For instance, naphthalene can be formed by the addition of $\mathrm{C}_{3} \mathrm{H}_{3}$ on benzyl radical $\left(\mathrm{c}-\mathrm{C}_{6} \mathrm{H}_{5} \mathrm{CH}_{2}\right)(\mathrm{R} 38)$ [211]:<smiles>[R19][18c]1ccc2ccc(CCC#CCc3ccccc3)cc2c1</smiles>

D'Anna and Violi [211] proposed the sequential addition of $\mathrm{C}_{3} \mathrm{H}_{3}$ to phenyl radicals, resulting in the formation of phenanthrene (R39).

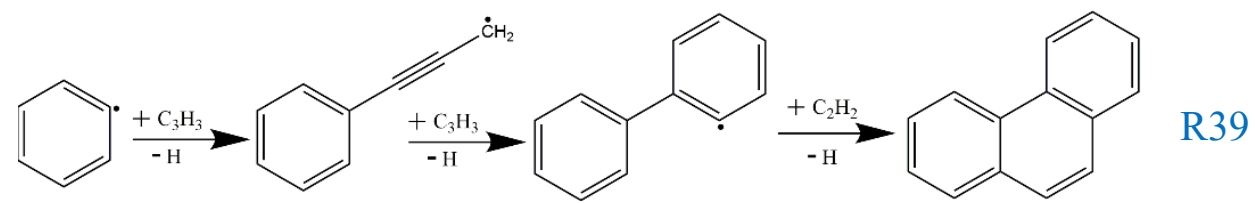

Raj et al. [212] recently recommended a PAH growth pathway from naphthalene to pyrene (R40) through $\mathrm{C}_{3} \mathrm{H}_{3}$ addition, and they provided kinetic parameters for each involved elementary reactions through theoretical computations based on the density functional theory (DFT). It was further suggested that analogous pathways and kinetic rates can be extrapolated to larger PAH molecules that have a zigzag site similar to naphthalene [212].

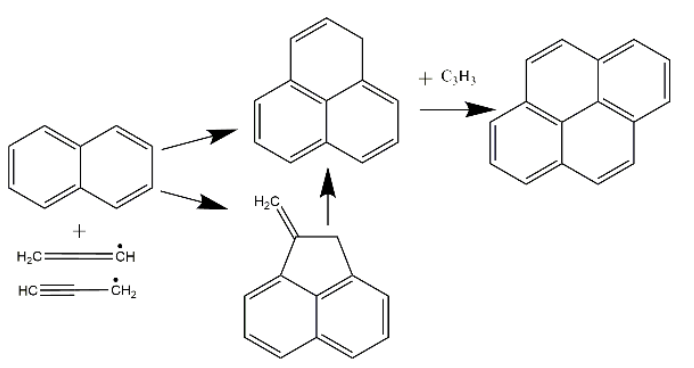

Aromatic radical substituted propargyl radicals were proposed to contribute to PAH growth by Stein et al. [153]. In particular, terphenyl (i.e., diphenylbenzenes, $\mathrm{C}_{18} \mathrm{H}_{14}$ ) can be formed through the recombination of phenyl-substituted $\mathrm{C}_{3} \mathrm{H}_{3}$ radicals ( $\left.\mathrm{R} 41\right)$. Note that further dehydrogenation will lead to the fused-ring structure triphenylene $\left(\mathrm{C}_{18} \mathrm{H}_{12}\right.$, a flat $\mathrm{PAH}$ consisting of four fused benzene rings). 


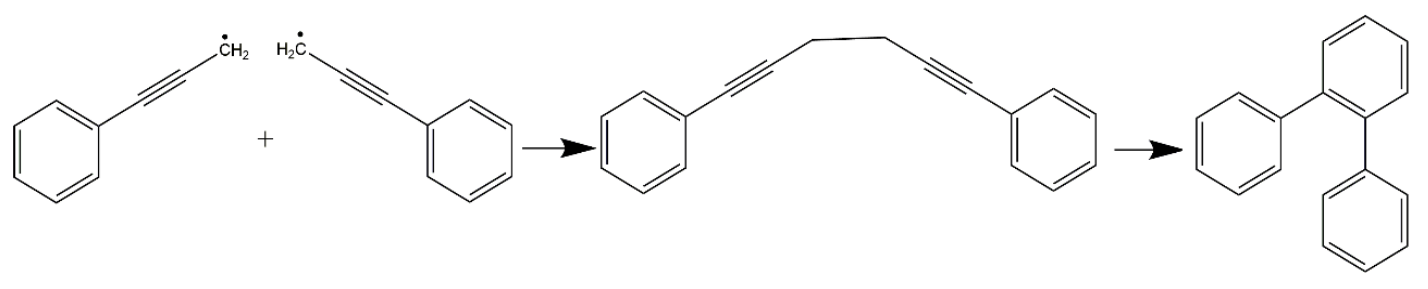

Another important RSR, cyclopentadienyl radical $\left(\mathrm{c}-\mathrm{C}_{5} \mathrm{H}_{5}\right)$, was shown to contribute to PAH growth. Besides its self-recombination to form naphthalene (R19), its addition to indenyl radical (c$\left.\mathrm{C}_{9} \mathrm{H}_{7}\right)$ can lead to the formation of phenanthrene (R42) [154]:<smiles>[R12]c1cccc2ccc(CC3C=Cc4ccccc4C3)cc12</smiles>

Indenyl radical can further contribute to pyrene formation through self-combination, or addition to benzyl radical, the global reaction of which was included in the kinetic network of Raj et al. [212].

It can be inferred from the above discussions that there exist many different pathways to PAH growth, and the relative importance of each individual pathway depends on fuel molecular structures as well as flame environments. In addition, resonantly stabilized radicals such as propargyl, substituted propargyl, cyclopentadienyl, benzyl and indenyl radicals are important growth agents. Most of these reactions are proposed only as global reactions so that further study is required for detailed theoretical analyses of the related elementary reactions to provide more accurate and pressure-dependent product channels and rate constants. On the other hand, statistical methods (i.e., kinetic Monte-Carlo) that allow PAHs to grow indefinitely by employing different classes of reactions in a jump manner [213-217], may be more practical in the context of soot simulation. This will be discussed further in Section 2.3 on soot surface growth, as recent models employ a chemical analogy [218] of soot with large PAHs, considering soot particles to be composed of stacks of PAHs and surface growth can be described by PAH planar or spatial growth at edge sites. 


\subsection{Soot nucleation}

Soot nucleation refers to the transition of gas-phase molecular soot precursors to condensed-phase carbon materials. According to Frenklach [122] and many other researchers, this transition is probably the least known process of soot formation. Nevertheless, extensive research in the last few decades has made it convincing that high molecular weight PAHs are the dominant-if not the sole-class of molecular precursors for soot nucleation. The evidence includes transmission electron microscopy (TEM) images of soot indicating ordered structures in soot particles, believed to arise from a stacking of PAHs to form parallel layers [219]. However, definite physicochemical mechanisms explaining how the first soot particles nucleate out of PAHs under flame conditions remain research in progress.

Various conceptually different pathways for soot nucleation have been suggested [18] and are briefly discussed here. More detailed analysis can be found in the review by Wang [18]. The first pathway (with some variation in details) generally concerns the formation and growth of fullerenelike structures. In the famous graphite laser vaporization experiments by Kroto et al. [220] in which Buckministerfullerene $\left(\mathrm{C}_{60}\right)$ was discovered, the decrease in the concentrations of other large $\mathrm{C}_{n}$ clusters (except $\mathrm{C}_{60}$ ) was observed with the increase in reaction residence time. The authors proposed that the consumption of these more reactive (compared to the remarkably stable $\mathrm{C}_{60}$ ) large carbon clusters are due to their interactions with the abundant small carbon species to form soot. It was further argued that, at typical flame temperatures where dehydrogenation are favored, carbon tends to form stable spherical shells to satisfy the valence requirements and curved PAHs with five-member rings can contribute to the generation of the spheroidal structures [221]. Based on this reasoning, an icospiral mechanism was proposed in which the formation of soot is described as a consequence of faulty growth of fullerene. The error during fullerene formation prevents cage closure, instead it leads to further molecular growth to onion-like structures [221, 222]. Figure 4a provides a schematic for this process. The icospiral mechanism suggests fullerenic structures to be precursors for soot, however, it should be noted that although the authors wish to generalize soot formation in flame 
conditions, this mechanism was based on experiments performed for a pure carbon system. Homann and coworkers [223-226] studied fullerene and soot formation in premixed flames using MBMS with photon ionization and observed that whenever soot or fullerene are formed, negative ions, covering a continuous mass spectrum up to 2,000 amu, are present. Presumably, this indicates the presence of aromers (aromatic oligomers), which were assumed to be products of PAH-PAH reactions [222, 225]. It was further argued that aromers possess many five-member rings (to explain their electron affinity) and have curved structures facilitating hydrogen loss, the features of which suggest their possible role as fullerene/soot precursors [222]. Homann explained further that once aromers are formed, the conditions of flame temperature, pressure, and intermediate species' concentration will determine whether they continue to react with unsaturated hydrocarbons $\left(\right.$ e.g., $\left.\mathrm{C}_{2} \mathrm{H}_{2}\right)$ to form soot or undergo unimolecular reactions toward cage closure to form fullerene. In conventional combustion environments, where hydrocarbons are burned in air (fuel rich) at atmospheric or elevated pressures, unsaturated small hydrocarbons are abundant, favoring soot formation [222]. The pathway from aromers to soot is schematically shown in Figure $4 b$.

(a)

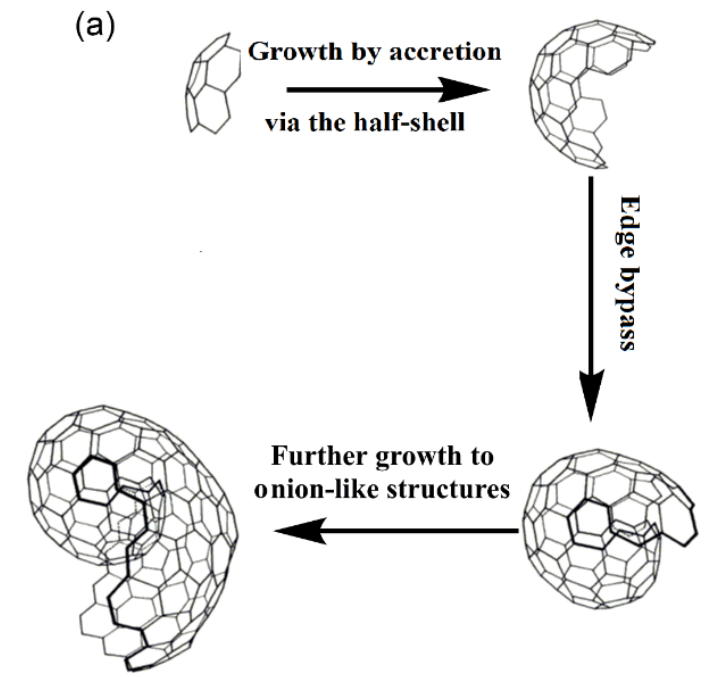

(b)

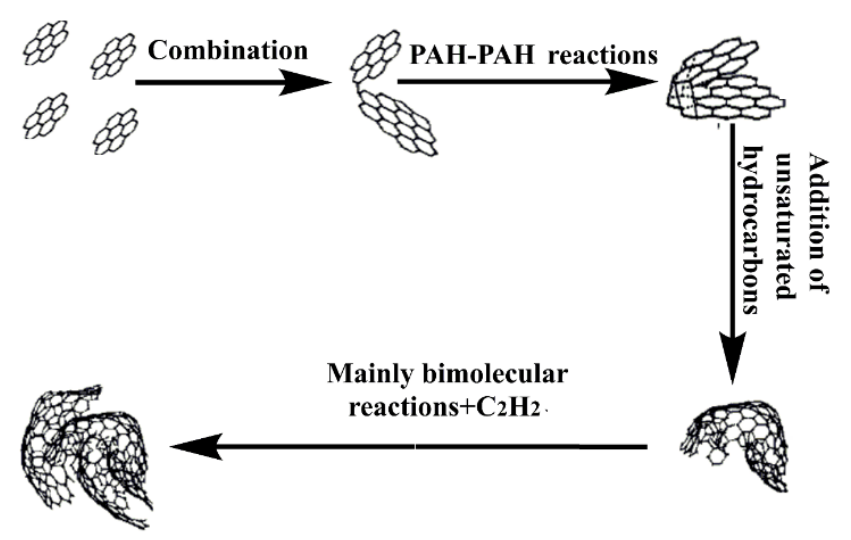

Figure 4. Schematic of soot formation through icospiral mechanism (a), adapted from Kroto [227] with permission of the AAAS, and from aromatic oligomers (b), adapted from Homann [222] with permission of John Wiley and Sons. 
Another nucleation pathway is related to the purely chemical growth of soot nuclei from PAH species. D'Anna and coworkers [125, 215] proposed a growth mechanism in which PAHs chemically coalescence with each other to form three-dimensional, cross-linked, ring-ring aromatic structures. With continuous mass and size growth of these structure, the properties of the condensed phase can finally be acquired. This process can also be schematized by the following repetitive reaction sequences $[125,215]$ :

$\mathrm{A}_{\mathrm{i}}+\mathrm{H} \rightleftharpoons \mathrm{A}_{\mathrm{i}^{-}}+\mathrm{H}_{2}$

$\mathrm{A}_{\mathrm{i}^{-}}+\mathrm{A}_{\mathrm{j}} \rightleftharpoons \mathrm{A}_{\mathrm{i}^{+j}}+\mathrm{H}$

where $A_{i}$ represents $P A H$ species and $A_{i-}$ their radicals and the subscript $i($ or $j, i+j)$ indicates the size of the PAHs. Note that for such a kinetic scheme-if steady state assumption is invoked for the radical species-the rate of aromatic growth can be expressed as $k_{44}\left[\mathrm{~A}_{\mathrm{i}}\right] K_{\mathrm{eq} \_43}[\mathrm{H}] /\left[\mathrm{H}_{2}\right]$, where $k_{44}$ is the rate constant for R44 and $K_{\text {eq_ }} 43$ is the equilibrium constant for R43. It can immediately be seen that the growth rate is dependent on the concentration ratio between $\mathrm{H}$ radical to $\mathrm{H}_{2}$ molecules. Therefore, it is reasonable to expect that a flame environment rich in $\mathrm{H}$ radicals is required in order for this chemical growth mechanism to be efficient.

In yet another pathway advocated by Frenklach and Wang [198], soot nucleation was explained to occur through physical sticking of PAHs. As the size of the aromatic species is increased beyond a certain critical value (via molecular chemical growth), they collide and begin to stick together to form PAH dimers, which continue to collide with other PAH molecules to form trimers and so on. From energy considerations, the most probable orientations for successful physical sticking are those leading to the formation of staked clusters. In this way, the PAH stacks become increasingly larger and eventually assume the properties of solid particles.

It is probably safe to say that none of the above pathways provides a universal or consistent explanation for the soot nucleation processes observed in different flame conditions. For instance, it has been argued that, from a kinetic point of view, the rates of the chemical reactions leading to 
fullerenic growth are too slow to explain the observed time scale of soot inception in experiments [198, 228]. Also, experimental diffraction patterns of soot particles were reported to suggest the presence of stacked turbostratic benzenoid arrays (as opposed to the spiraling structure) [228], which were also supported by soot images taken with TEM [229]. Recent measurements on soot particle sizes in premixed flames confirm the dominance of bimodal size distributions [48, 49, 230-233], evidence against the icospiral nucleation pathway. The underlying reasoning was provided by Wang [18] as follows: The solution of the Smoluchowski coagulation equation with persistent source of nucleation shows that the particle size distribution function would quickly evolve into a unimodal if the rate of nucleation is first order with monomer concentration $[18,234]$, which is the case for icospiral nucleation mechanism. As a result, this disagreement with the experiments in terms of size distribution excludes the icospiral mechanism to be the dominant pathway for soot nucleation, at least for the premixed flame conditions investigated.

As previously mentioned, the PAH chemical coalescence pathway has its limitations in accounting for persistent soot nucleation in regions where the concentration of $\mathrm{H}$ radical is low. For molecular growth reactions to occur between PAH species (R44), one of the PAH molecules must be activated to form aryl radical, which is typically achieved through $\mathrm{H}$-abstraction reaction involving $\mathrm{H}$ radical (R43). As a result, it is expected that in the post-flame zone of fuel-rich premixed flames (or the fuel side of non-premixed flames where H radicals exist only in very low concentrations), the rates of soot nucleation through chemical coalescence may be negligible. However, experimental data provide evidence that soot nucleation continues in such regions [230], suggesting that other nucleation mechanisms are responsible.

The primary issue concerning the PAH physical dimerization pathway is the contradiction between the need for large PAHs for dimer stability and the difficulty in forming these large PAH molecules. For the sticking of two PAH monomers to be successful in high temperature flame environments, the electrostatic and dispersive binding forces must be strong enough to counteract the entropy resistance 
for binding. This translates to the fact that the colliding PAH monomers must reach a rather large size if dimer formation is to be realized. A recent molecular dynamic study [235] confirmed that PAH dimer stability is controlled by monomer mass and solvent-accessible surface area, accompanied by the discovery that-in the case of heteromolecular dimerization-dimer stability is determined primarily by the size of the smaller monomer. Direct measurement of pyrene (four-ring PAH) dimerization rates in low temperature supersonic jets demonstrated pyrene dimerization, which is frequently modeled for nucleation, cannot proceed to a significant level at flame temperatures [236]. Similar conclusions were reached by a recent molecular dynamic study, showing that pyrene dimerization at $1500 \mathrm{~K}$ is almost impossible [237]. It was further estimated that a 3.6 bar partial pressure of pyrene is required for $1 \%$ pyrene to be converted to dimers at $1500 \mathrm{~K}$, while in practical hydrocarbon flames, the volume fractions of pyrene are generally on the order of $10^{-6}$. In fact, based on theoretically calculated binding energy data [238-240], assuming equilibrium kinetics, a size of circumcoronene $\left(\mathrm{C}_{54} \mathrm{H}_{18}\right)$ is required for successful dimerization at typical flame temperatures [18]. Non-equilibrium effects were also discussed by Schuetz and Frenklach [241], who performed molecular dynamics simulation of pyrene dimerization with on-the-fly quantum forces. They showed that a rovibrationally excited dimer can be formed from two pyrene molecules with a lifetime significantly longer than the collision time scale, exhibiting the possibility of further chemical reactions leading to the formation of a covalent bond between the two PAH monomers. An unresolved issue of these postulates is whether the excited dimer/initial clusters can survive constant non-reactive collisions during their lifetimes.

On the other hand, experiments have shown that peak concentrations decrease exponentially for increasingly larger PAHs. For instance, in a typical ethane counterflow diffusion flame [242] at atmospheric pressure with a benzene mole fraction on the order of $10^{-4}$, the mole fractions for the two-ring naphthalene and four-ring pyrene dropped to $10^{-5}$ and $10^{-6}$, respectively. In fact, it has been pointed out that in typical sooting premixed flames, the concentrations of PAHs decreased by roughly 
one order of magnitude with the growth of every two peri-condensed rings [18]. Therefore, it is expected that the concentrations of large PAHs (i.e., $\mathrm{A}_{19} \mathrm{C}_{54} \mathrm{H}_{18}$ ) will be too small to account for typical values of soot number density observed in actual sooting flames.

There is also an experimental evidence that raises questions about nucleation from pyrene. Recent aerosol mass spectrometer studies on extracted soot particles from a $\mathrm{C}_{2} \mathrm{H}_{4} / \mathrm{C}_{2} \mathrm{H}_{2}$ counterflow diffusion flame showed that species with mass 202 amu (pyrene or its isomers) contribute a very small fraction of the high-mass species [136, 137]. Even for the mass peak of $202 \mathrm{amu}$, the photo-ionization efficiency curve (PIE, discussed later in the section on experimental method) showed that it was most likely contributed by isomers of pyrene, leading to the tentative conclusion that PAH species other than the Stein stabilomers [135] may be important for soot nucleation.

The insufficiency of the above soot nucleation hypothesis in explaining experimental observations motivates the investigation of additional pathways for nascent soot formation; several research groups have made significant efforts to search for additional/alternative nucleation schemes. For example, through molecular dynamics simulations, Violi and coworkers [243, 244] showed that the aliphatic chains attached to aromatic rings may be important for facilitating the physical sticking of PAHs into clusters at high temperatures, by accommodating the excessive collision energy into the additional internal vibrational modes of these side chains.

Considering the experimental observation that, in addition to PAH clusters held together by physical forces, aromatic moieties linked by chemical bonds are also present in high temperature region of a counterflow diffusion flame [89], Mao et al. [245] pointed out the potential importance of chemical nucleation for the formation of incipient soot formation. They investigated the soot nucleation process from a pool of PAH molecules of various sizes using the reactive force-field (ReaxFF) molecular dynamic simulation, which has the capability to account for both physical and chemical reactions. Results showed that at low temperatures, even PAHs as small as naphthalene can nucleate into stacked structures via physical interactions. Nevertheless, at typical temperatures in 
sooting regions of hydrocarbon flames (i.e., $\sim 1600 \mathrm{~K}$ ), physical sticking of PAHs was not possible except for very large molecules (e.g., circumcoronene, $\mathrm{A}_{19}$ ), which is consistent with the conclusion of Sabbah et al. [236]. With a further increase in temperature to above $2500 \mathrm{~K}$, PAHs became chemically active through dehydrogenation (i.e., formation of aryl radicals). Subsequent PAH fragmentation produced polyacetylenic-like radicals or small molecules which further contributed to molecular growth. Specifically, both PAH clusters connected with a carbon bridge (i.e., a covalent bond with polyacetylenic chains) at the edge, and fullerenic structures were observed. This study essentially supports the chemical growth pathway proposed by D'Anna and coworkers [125, 215]. However, the high temperature $(>2500 \mathrm{~K})$ required for $\mathrm{H}$-abstraction through dehydrogenation may not fully explain the ubiquitous soot formation at much lower temperatures $(\sim 1500 \mathrm{~K})[246,247]$.

Motivated by the prediction that the zigzag edges of graphene, or finite-sized graphenes, contain radical characteristics originating from localized $\pi$-electrons, Wang [18] pointed out the potential role of aromatic $\pi$-radicals in strengthening the binding in PAH clusters. In a step forward, Zhang et al. [248] performed quantum chemistry calculations to investigate the effects of $\pi$-electrons in covalent bond formation during PAH dimerization. The bond nature (i.e., number of bonds and sites for bonding) was systematically studied for dimerization of both identical and different rectangular PAHs. Their results confirmed the possibility of covalent bonding, and that the radical character dictates the number of bonds formed, while the aromaticity of the individual six-member ring determines the bond sites. This implies that bonding is more likely to form in rings with the largest aromaticity. It must be pointed out, however, the discussion of Zhang et al. [248] was based on a zero-temperature scenario, while at combustion relevant temperatures the dimers formed were found to be thermodynamically unstable [249], primarily due to the large decrease of dimerization entropy. Nevertheless, the authors considered that the lifetime of dimers may be longer than the molecular collision time scale so that they, once formed, could still contribute to the nucleation of soot particles. 
It remains to be proved whether these unstable dimers can survive constant collisions from molecules of other combustion gases.

The role of cation- $\pi$ in enhancing the stability of PAH clusters, inspired by previous astrophysical studies in which the existence of PAHs in the interstellar medium [250-252] and the enhanced PAH binding by a metal cation $[253,254]$ were discussed, has recently drawn attention from researchers on soot. Recognizing that the most abundant cations in hydrocarbon flames are $\mathrm{CHO}^{+}, \mathrm{C}_{3} \mathrm{H}_{3}{ }^{+}$and $\mathrm{H}_{3} \mathrm{O}^{+}$resulting from chemi-ionization [255, 256], Chen and Wang [257] performed ab initio and density functional theory calculations to investigate the binding energies and structures of complexes formed from the association of these chemi-ions and 1 to 4 ring PAHs. Their results showed that although the cation- $\pi$ interactions make a positive contribution, the cation-enhanced bindings are still too weak to account for soot nucleation from PAH clusters. However, similar studies on the interactions between cations of transition metals and benzene molecules [258, 259] have shown very strong binding energies among the complexes $\mathrm{M}^{\mathrm{Z}+}(\mathrm{Bz})_{n}$, where $\mathrm{M}^{\mathrm{z}^{+}}$is the metal cation (i.e., $\mathrm{Fe}^{2+}$, $\left.\mathrm{Co}^{2+}, \mathrm{Cu}^{2+}\right), \mathrm{Bz}$ refers to benzene, and $n$ is the number of benzene molecules in the complex. Such enhanced binding strength indicates the potential of $\mathrm{M}^{\mathrm{Z}}(\mathrm{Bz})_{n}$ to form the first soot nucleus [258]. This is especially relevant considering many trace metals present in practical hydrocarbon fuels, as well as in engine lubricating oils. However, it must be pointed out that although theoretically plausible, direct experimental evidence has yet to be produced for the facilitation of $\mathrm{M}^{\mathrm{Z}+}(\mathrm{Bz})_{n}$ in soot nucleation, despite the fact that the effect of metal addition in soot formation was studied decades ago [260]. The problem is complicated because, besides its potential role in nucleation, a metal additive can also catalytically reduce the formation of molecular soot precursors [261, 262], enhance the oxidation of soot once formed [45, 261, 263], and suppress particle-particle coagulation due to repulsive forces $[260,264]$.

More recently, Kraft and coworkers [265, 266] noticed that curved PAHs (cPAH), with pentagonal ring integrated in their structures, are featured with significant molecular flexoelectric dipole 
moments. They further investigated the possibility of dipole-induced homodimerization of cPAHs, only to find that the binding energy between cPAHs is comparable with or even lower than that of planar PAHs [267]. This is because the steric effects of cPAHs caused a reduction in dispersion contribution, and thus cancelled the effects of dipole-dipole interactions [267]. Nevertheless, it was shown that cPAHs can bind strongly with chem-ions, the interactions between which are long-ranged and can be significant especially in combustion systems where concentrations of relevant species are low [268]. The proposal that the interaction between cPAHs and ions may play an important role in soot formation [266, 268] was also supported by recent experimental results that demonstrated enhanced physical clustering with cations [54, 269]. Nevertheless, further theoretical and experimental efforts are needed to confirm this ion-induced nucleation mechanism.

From a modelling perspective, soot nucleation must be quantitatively accounted for in any predictive soot models because it bridges the treatment of gas phase and surface reactions, in addition to serving as a turning point beyond which particle dynamics is initiated. Historical treatments are largely empirical and include the one-step nucleation from $\mathrm{C}_{2} \mathrm{H}_{2}$ [270], as shown in $\mathrm{R} 45$, and that from benzene in R46 [271]. Many modern soot inception models follow the work of Frenklach and Wang [198] and describe soot nucleation with irreversible [198] or reversible dimerization [272] of peri-condensed aromatic species, most notably pyrene molecules. Although these models successfully explain many experimental observations, there has been compelling evidence suggesting that these dimers, linked by physical forces, are not stable enough at flame temperature to accommodate further growth [243], and that chemical bonds between monomers are essential for the formation of stable PAH clusters. The main challenges for current chemical nucleation mechanisms, mentioned above, are that they involve reactions with high-energy barriers (leading to slow reaction rates), which are not perfectly compatible with certain experimental observations. One example is the experimentally-observed persistent soot nucleation in the post-flame zone of rich premixed flames, which is characterized by low temperature and low concentrations of reactive radicals (i.e., $\mathrm{H}$ atom). 
$\mathrm{C}_{2} \mathrm{H}_{2} \rightarrow 2 \mathrm{C}_{\text {soot }}+\mathrm{H}_{2}$

$\mathrm{C}_{6} \mathrm{H}_{6} \rightarrow 6 \mathrm{C}_{\text {soot }}+3 \mathrm{H}_{2}$

Recently, a new nucleation mechanism, termed clustering of hydrocarbons by radical-chain reactions (CHRCR) was proposed by Johansson et al. [138]. The CHRCR mechanism highlights the importance of chain propagation of resonantly stabilized radicals (RSR). In particular, the molecular growth process initiates with repetitive additions of $\mathrm{C}_{2} \mathrm{H}_{2}$ or vinyl radical $\left(\mathrm{C}_{2} \mathrm{H}_{3}\right)$ to $\mathrm{RSRs}\left(\mathrm{e} . \mathrm{g}\right.$., $\mathrm{C}_{3} \mathrm{H}_{3}$ or $\mathrm{c}_{-} \mathrm{C}_{5} \mathrm{H}_{5}$ ), generating new RSRs with increasingly higher molecular weights. These RSRs can subsequently cluster with different types of hydrocarbons, including stable PAHs and unsaturated aliphatic species, while preserving the radical nature. As such, molecular growth can proceed without the need for high energy barrier activation reaction, leading finally to covalently-bonded large clusters (incipient particles). In this way the CHRCR mechanism avoids the challenges for existing chemical nucleation mechanisms. It also overcomes the problem of insufficient concentration of large PAHs because RSRs can cluster with a wide range of different hydrocarbons [138]. The CHRCR mechanism was motivated partially by experimental observations (through VUV-MS measurements) of a class of increasingly larger RSRs in sooting flames. Note that the chain propagation reactions involving RSRs has been confirmed by quantum computations [138]. However, detailed rates for the various proposed radical chain propagation reactions still need to be determined so that a complete mechanism can be assembled to test the capabilities of the CHRCR mechanism in predicting the formation of large molecular species and soot [273].

2.3 Soot surface growth, particle coalescence, and agglomeration

Surface growth refers to the process in which gaseous species attach to the surface of pre-existing soot particles, and their subsequent incorporation into the particulate phase [119,274]. It is generally believed that although inception is the first step for soot formation, providing the nucleus, the majority of soot mass comes from surface growth [274]. For instance, the results of a numerical simulation study on soot formation in ethylene/air coflow diffusion flames [72] showed that the radially 
integrated soot mass growth rate from surface reactions were more than an order of magnitude higher than from new particle inception. Similarly, Kennedy [275] showed that more than $90 \%$ of the final soot volume is produced from surface growth in counterflow diffusion flames.

Although detailed physicochemical processes of surface growth are still not fully understood, semiempirical descriptions of its rates were proposed decades ago, initially for premixed flames [274, 276], and later for diffusion flames as well [277-279]. These early studies generally showed a firstorder kinetics with the gas phase soot growth agent, for which acetylene was considered to be a primary candidate [276]. The rate of the mass adding reaction $\mathrm{C}_{2} \mathrm{H}_{2}+\mathrm{nC}_{\text {soot }}=(\mathrm{n}+2) \mathrm{C}_{\text {soot }}+\mathrm{H}_{2}$ then took the simple form of $\omega_{\mathrm{g}}=k(T) f(S)\left[\mathrm{C}_{2} \mathrm{H}_{2}\right]$, where $\omega_{\mathrm{g}}$ is the soot growth rate, $k(T)$ is the rate constant dependent on temperature, $T$, and $f(S)$ is a function of soot surface area [270]. The authors argued that because active sites may decrease in concentration with the extent of soot growth due to surface aging $[119,129], \omega_{\mathrm{g}}$ should not be directly proportional to $S$. They went on to propose, ad hoc, that the number of active sites is proportional to the square root of $S$, and the soot growth rate can be written as:

$$
\omega_{\mathrm{g}}=k_{\mathrm{g}}(T)\left[\mathrm{C}_{2} \mathrm{H}_{2}\right] \sqrt{\pi\left(\frac{6 M_{C}}{\pi \rho_{C}}\right)^{2 / 3}}[\mathrm{C}]^{1 / 3}(\rho N)^{1 / 6}
$$

where $M_{\mathrm{C}}$ is the molar weight of carbon, [C] is the molar concentration of solid carbon, $N$ is the number of particles per unit mixture mass, and $\rho_{\mathrm{C}}$ is the density of soot [270]. Later experimental determination of mass growth rates following streamlines in coflow diffusion flames indicated that $\omega_{\mathrm{g}}$ was dependent on acetylene concentration raised to a power of $n$, slightly larger than unity [277, 279]. This difference, as compared to Eq. 1, was thought to be caused by soot oxidation (discussed in detail in a subsequent section) which occurred during the experiment, thereby masking the net soot growth rate [279]. After accounting for soot oxidation, $n$ was found to be close to unity [279].

More physically based description of surface growth was stimulated by the hypothesis of chemical similarity between soot particles and large PAHs that provided a possibility to treat surface reactions through elemental reactions [122]. In this regard, the surface analogy of the gas phase HACA 
mechanism-that is the surface-HACA mechanism-was proposed to describe soot mass growth through the following reaction sequences [198, 280]:

$\mathrm{C}_{\text {soot }}-\mathrm{H}+\mathrm{H} \rightarrow \mathrm{C}_{\text {soot }} \cdot \mathrm{H}_{2}$

$\mathrm{C}_{\text {soot }}+\mathrm{H} \rightarrow \mathrm{C}_{\text {soot }}-\mathrm{H}$

$\mathrm{C}_{\text {soot }}+\mathrm{C}_{2} \mathrm{H}_{2} \rightarrow \mathrm{C}_{\text {soot }}-\mathrm{H}+\mathrm{H}$

where $\mathrm{C}_{\text {soot }}$ is bulk soot and $\mathrm{C}_{\text {soot }}-\mathrm{H}$ represents a saturated armchair (discussed later) surface site, which needs to be activated (via hydrogen abstraction) from the aryl radical site $\mathrm{C}_{\text {soot }}$ before a $\mathrm{C}_{2} \mathrm{H}_{2}$ molecular can be added to increase the mass/size of the bulk carbon particle. The rate of hydrogen abstraction reaction (R47) can be obtained by kinetic analogy of similar reactions with aromatic species (e.g., benzene).

In addition to $\mathrm{H}$ radical, $\mathrm{OH}[146,280], \mathrm{CH}_{3}, \mathrm{C}_{2} \mathrm{H}, \mathrm{C}_{3} \mathrm{H}_{3}$, and other hydrocarbon radicals, may also initiate hydrogen abstraction reactions. Hwang and Chung [281] proposed and tested a modified surface-HACA growth mechanism, emphasizing the roles of $\mathrm{CH}_{3}, \mathrm{C}_{2} \mathrm{H}, \mathrm{C}_{3} \mathrm{H}_{3}$ in the surface site activation reactions to explain measured soot growth rates in CDFs, where $\mathrm{H}$ radical concentration was relatively low, for example in the relatively low temperature soot growth region in SF counterflow diffusion flames.

A parameter $\alpha$, having a physical representation of the fraction of surface sites available for chemical reactions, was introduced by Frenklach and Wang [198] to account for the surface aging of soot particles. In principle, the extent of soot aging, and thus the value of $\alpha(\leq 1)$, should depend on both particle size and soot surface temperature [187]. By testing several different premixed flames, an empirical correlation of $\alpha$ was proposed:

$$
\alpha=\tanh \left(\frac{a}{\log \mu_{1}}+b\right)
$$

where $\mu_{1}$ is the first moment of soot size distribution function and $a$ and $b$ are linear functions of temperature, which could be determined by matching with experiments. These expressions were developed based on soot measurement data for premixed flames; and there are other relations that 
were used for different flame configurations [282, 283]. The Arrhenius type expression of $\alpha=\min$ $(c \times \exp (d / T), 1)$, in particular, was frequently used in coflow diffusion flames [71,284], where $c$ and $d$ are fitting constants. The value of $\alpha$ was also sometimes assumed to be a constant, for example in a previous counterflow flame simulation [78], unity value was used and a much smaller constant of 0.078 was employed by Dworkin et al. [282]. Note also that in the surface-HACA growth mechanism, the $\mathrm{C}_{2} \mathrm{H}_{2}$ addition step (R49) was assumed to be irreversible, motivated by the fact that the dependence of thermal decomposition of the surface intermediates on flame temperature would be overpredicted if reversible reaction was included $[122,198]$. Nevertheless, the complete exclusion of reversibility then underestimated the temperature dependence observed experimentally and the inclusion of $\alpha$ can in a way be considered a remedy [122]. A theoretical study was recently performed [285] to microscopically investigate the surface properties of equilibrated pyrene and coronene clusters [239, 286] (as a representation of nascent soot particles), demonstrating a decreasing (increasing) trend of surface availability with increased particle size (temperature). Note that the relationship with temperature is different from the common concept of a decreasing $\alpha$ with the increase in temperature; the authors explained this difference by pointing out that surface availability is a local property, while $\alpha$ is a global property of the soot ensemble. An upper limit of $0.8 \sim 0.9$ for $\alpha$ was also proposed [285], considering the pocket surface is inaccessible for gaseous species because of their shorter life time as compared to surface reaction time.

Besides $\mathrm{C}_{2} \mathrm{H}_{2}$ addition, the importance of $\mathrm{PAH}$ addition on particle surface in soot mass growth was also demonstrated [272, 287-289]. A modelling study on soot formation in a high-pressure coflow diffusion flame [72] showed that at pressures higher than 6 bar, the soot growth rate through PAH addition can even overtake the surface-HACA growth rate. D'Anna et al. [290] also showed that a physical mechanism such as PAH addition is more likely to contribute to nanoparticle formation and growth in the pyrolytic region of soot formation in $\mathrm{CDF}$, where temperatures and $\mathrm{H}$ radical concentration were relatively low. 
From a first principle, the types and rates of reactions on soot surfaces may depend on many parameters including temperature, size, morphology, detailed chemical composition, type, and steric confinements of reactive sites on the particles (note that the original surface-HACA mechanism [198] considered only the armchair site, and site density was pre-specified, while surface reactions on other types of sites may also be possible [291]). In addition, more recent experimental investigations in premixed flames provide both direct (aerosol mass spectrometry, Fourier-transform infrared (FTIR) and Raman spectroscopy) and indirect (TEM and atomic force microscope (AFM) [97]) evidence for the existence of aliphatic chains covering the aromatic core of nascent soot particles. These findings necessitate the description of mass growth reactions on different surface sites so that the chemical and structural details of soot particles must be tracked in soot models [18]. Toward this goal, and considering the chemical analogy of soot with PAHs (as pioneered by Frenklach [218]), Singh and Frenklach [292] and Kraft and coworkers [213, 216, 293] differentiated soot surface sites (as shown in Figure 5) and developed detailed surface mechanisms that take into consideration the different reactions on the various sites. In addition to conventional $\mathrm{H}$ abstraction and $\mathrm{C}_{2} \mathrm{H}_{2}$ addition reactions in an armchair site, as discussed in [280], surface reaction can also include the formation of a fivemember ring by the addition of $\mathrm{C}_{2} \mathrm{H}_{2}$ at zigzag sites and its desorption [294], free-edge ring growth (via $\mathrm{C}_{2} \mathrm{H}_{2}$ ) and desorption [218], five- to six-member ring, as well as six- to five-member ring conversion [291, 295, 296], five- and six-member bay closure [295, 297], five-member ring migration [291, 295], benzene addition to form bi-phenyl-like structures and so on. More details of the many surface reaction classes can be found in Refs. [213, 216, 298, 299]. These reactions can be followed in detail for a single seed PAH molecule (e.g., pyrene) using a Monte Carlo algorithm, resulting in the kinetic Monte Carlo aromatic site (KMC-ARS) model [216], which can specifically track the shape and chemical identity of the growing PAH. Note that soot particles were modeled by Kraft and coworkers $[216,300]$ to be composed of planar PAHs, which contain bulk carbon atoms and edge carbon atoms. It is on these edge atoms (i.e., soot sites) that surface reaction can take place. 


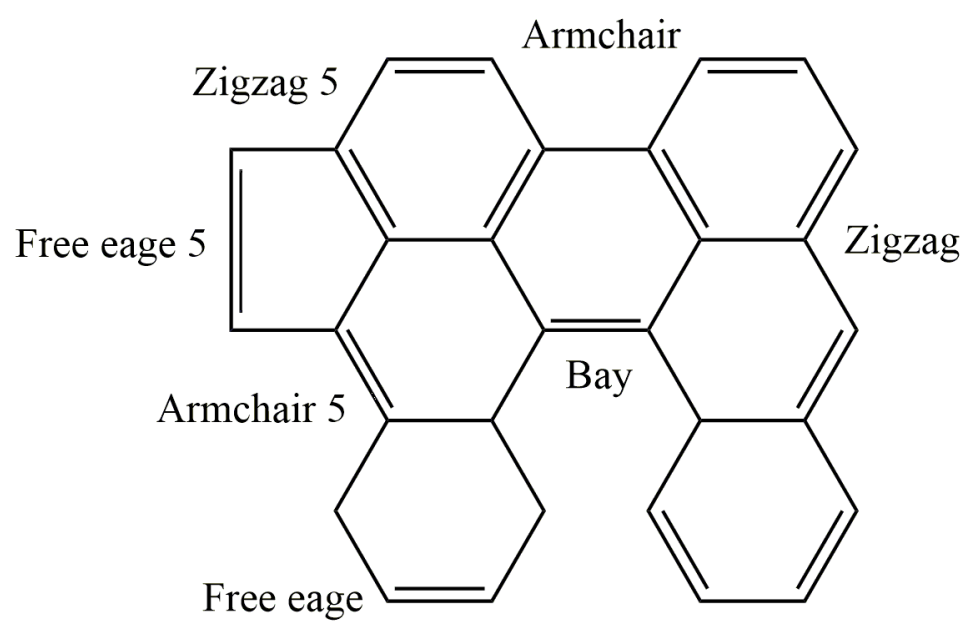

Figure 5. Typical PAH structure showing different types of surface sites. Five indicates that the site involves a five-member ring. Adapted from Singh et al. [292] with permission of Elsevier.

The 3-D growth of PAH structures was also studied by Frenklach and coworkers [292, 299, 301, 302] and Violi and coworkers [303-305], partially motivated by the fact that the originally planar PAH structure can develop curvatures by incorporating five-member rings [228]. Evidence was provided by high resolution TEM (HRTEM) images which showed curved PAH lattices [306, 307]. The presence of curvature in PAHs have implications in terms of soot inception, especially considering the potential interaction (yet to be explored) between polar curved PAHs and chemi-ions [308]. Particles consisting of curved PAH lattices are also shown to be more easily oxidized [309], and a recent soot growth model by Yapp et al. [310] managed to estimate PAH curvature with information about the number of five- and six-member rings in the PAH structure.

Although surface growth accounts for the majority of soot mass, ultimate particle size and morphology are largely influenced by particle-particle interactions (i.e., coagulation). Collisional interactions between particles can be generally classified into coalescence and agglomeration. Coalescence typically indicates the disappearance of the boundary between two particles in contact, resulting in a reduction of the total surface area; while agglomeration refers to the sticking of particles with point contact (conserving surface area). TEM images of nascent particles shows a spherical shape; while mature soot particles generally have nearly spherical primary particles collected into 
fractal aggregates with varying numbers of primary particles [311, 312]. The spherical nature of the nascent/primary particles is probably a major motivation for an early model [198] to treat particle coagulation as purely coalescent, i.e., colliding particles merging to form a larger spherical particle. However, later investigations [313-315] showed that sphericity can also be attained by point-contact particle aggregation with simultaneous surface growth. Under conditions where colliding partners were mostly small particles (in regions where soot inception rates were high and newly incepted particles abundant), surface growth effectively smoothed out the aggregates towards highly spherical particles. On the other hand, in environments where colliding partners were large (e.g. in the postflame zone of rich premixed flames), the smoothing effect of surface growth became relatively less important and a chain-like fractal was more likely to result.

Nevertheless, coalescent growth is still highly likely for nascent particles with liquid-like behavior. There is also experimental evidence that under high temperature conditions $(>1500 \mathrm{~K})$, even mature chain-like soot aggregate can experience in-particle sintering, and thus reduction of surface area and increased primary particle size [316]. In a recent detailed soot model [317], sintering was considered to be quasi-instantaneous for primary particles with a size smaller than a critical value $\left(D_{\mathrm{pp}, \text { crit }}\right)$, while for larger particles, an empirical model was used, with characteristic sintering time between two primary particles having an Arrhenius-type expression.

Complicated surface growth and particle-particle interactions would certainly result in polydispersed soot ensembles with complex particle size distributions (PSD). Recent research in highfidelity measurement of PSD using differential mobility analysis (DMA) (see e.g., [49, 52, 54, 97, 318-321], with a detailed discussion provided by Wang [18]) opened new possibilities for understanding the above-described processes through an inverse problem [18, 50, 322, 323]. The PAH-PP soot model proposed by Kraft and coworkers [300, 317] had several parameters, such as soot density, smoothing factor, pre-exponential factor, activation energy of sintering, and fractions of reactive sites, that were optimized by comparison against experimental PSDs. Through analysis of 
the PSD data obtained from AFM and scattering measurements, D'Alessio et al. [324] found that the sticking coefficient of coagulation (i.e., the actual measured coagulation rate divided by the theoretical value obtained from gas kinetic theory) for the nascent nanoscale organic carbon particle (NOC, $<3 \mathrm{~nm}$ ) was orders of magnitude smaller than the larger soot particles. Additional PSD measurements using both DMA and AFM confirmed that small particles have less propensity to coagulate at flame temperatures $[325,326]$. By comparing the measured and computed PSD, Sirignano and D'Anna [219] further demonstrated that coagulation efficiency depends on both particle size and temperature. Small particles had low coagulation efficiency even at room temperature and they decreased further with the increase in temperature. On the other hand, coagulation efficiency for large particles did not have strong temperature sensitivity and exhibited a high value for the entire temperature range examined.

\subsection{Soot oxidation and fragmentation}

Soot oxidation is a process that counteracts soot growth and it is also the primary mechanism for their removal from combustion exhausts. Therefore, a mechanistic understanding of soot oxidation is of both fundamental and practical importance. A comprehensive review of early experimental and kinetic studies on soot oxidation (before 2001) was provided by Stanmore et al. [327] and a recent informative account by Frenklach et al. [200]. It is generally recognized that the most important soot oxidants are $\mathrm{OH}$ radical and molecular $\mathrm{O}_{2}$, with contributions also from $\mathrm{O}$ atom, $\mathrm{H}_{2} \mathrm{O}, \mathrm{CO}_{2}$ and $\mathrm{NO}_{2}$ $[327,328]$. Note that $\mathrm{NO}_{2}$ can be particularly important at low temperatures under conditions like the regeneration of a diesel particulate filter (DPF) [329, 330].

At high temperature flame conditions, $\mathrm{OH}$ radicals [331-334] are particularly effective in oxidizing soot, and this can be especially true in CDFs, where concentrations of molecular $\mathrm{O}_{2}$ in the sooting zone are relatively low. From a modeling perspective, soot oxidation by $\mathrm{OH}$ was typically described by the collision of a soot surface site $\left(\mathrm{C}_{\text {soot }}-\mathrm{H}\right)$ with $\mathrm{OH}[198,280]$, subject to collision efficiency that can be determined experimentally [334-337]. A widely-used value of this collision efficiency is 0.13 , 
as obtained by Neoh et al. [334] as a lower bound value. D'Anna and coworkers [214, 215, 338, 339] also considered an activation energy for such reactions, which was estimated from similar reactions for benzene and PAHs. Although such relatively simple models have been successful in predicting experimental flame data, recent theoretical studies [340-342] found that the reaction of $\mathrm{OH}+\mathrm{C}_{\text {soot }}-\mathrm{H}$ (using benzene as a prototype) does not lead to CO expulsion, and thus was not considered to be a relevant soot oxidation pathway. Instead, $\mathrm{OH}$ reacting with armchair (oxidation more likely at armchair site $[343,344])$ soot surface radical site $\mathrm{C}_{\text {soot }} \cdot$ was shown to form various oxy-radicals, which then decomposed to eliminate CO. Therefore, in a most recent soot oxidation study [200], soot oxidation by $\mathrm{OH}$ was considered to occur only through its reaction with reactive radical surface sites. Soot oxidation was recently studied in an environmental TEM [345]. Although the feeding gaseous reactant was molecular $\mathrm{O}_{2}$, the high-energy electron beam of the TEM was seen to be very effective in ionizing $\mathrm{O}_{2}$ molecules. It was thus expected that the active soot oxidants were in fact radicals such as $\mathrm{O}$ atom or ion. The results confirmed that a $\mathrm{d}^{2}$-law also holds for surface oxidation of primary soot particles, regardless of their location inside the soot aggregate. The study also provided clear evidence of both the internal and surface burning modes of soot particles, confirming previous findings using conventional TEM images [346-348]. For mature particles with a thick graphite shell [349], surface oxidation dominates as it would be difficult for oxygen to diffuse across the outer shell into the core of the particles. These experimental results are believed to be helpful to model developments for soot oxidation.

Soot oxidation by molecular $\mathrm{O}_{2}$ has recently attracted significant research attention $[292,345,350$ 361], motivated at least partially by the issue of DPF regeneration. Early models described soot oxidation by $\mathrm{O}_{2}$ as a single step: $\mathrm{C}_{\text {soot }} \cdot+\mathrm{O}_{2} \rightarrow$ products, with rate coefficients taken by chemical analogy to the reaction of phenyl radical with $\mathrm{O}_{2}$ [280], later updated using rates calculated for $\mathrm{O}_{2}$ oxidation of pyrene radical (4-pyrenyl) to form phenanthrene radical (4-phenanthryl) [293]. However, this updated rate [293] was noted to be slow [362] so that additional detailed mechanistic studies were 
performed and results analyzed to identify more favorable oxidation pathways [351, 363, 364]. Sendt and Haynes [365-367] investigated $\mathrm{O}_{2}$ addition on different types of surface sites, providing understanding of the mechanism on $\mathrm{O}_{2}$-surface sites interactions. In their studies, however, multiple adjacent radical sites were involved, which may exist only in exceedingly small concentrations considering the fraction of radical sites among surface sites were already low $\left(10^{-2}\right.$ to $\left.10^{-4}\right)$. In this regard, Raj and coworkers used quantum calculations to re-examine reaction pathways of $\mathrm{O}_{2}$ oxidation at free-edge [362] and zig-zag edge sites [368]. Only one radical site was present in the model reactant, and the computed reaction pathways and high-pressure limit rate constants were provided for potential incorporation into detailed soot models.

Two prominent phenomena with respect to soot burnout in oxygen-containing environments have been observed experimentally: 1) Particles containing curved PAH structures oxidize notably faster than those composed of planar PAHs [309, 359, 369] and 2) Soot oxidation reactivity tends to decrease as oxidation progresses [370]. Interestingly enough, as explained by Singh and Frenklach [292], both these enhanced and reduced soot oxidation reactivity can be explained by the presence of five-member rings in soot structures. On one hand, quantum calculations showed that activation energy for the oxidation of curved PAH (represented by 1-corannulenyl radical with a five-member ring in the center) is notably lower than that of planar PAH (represented by 4-pyrenyl radical) [359]. Sterically-resolved kinetic Monte Carlo simulation on large graphene sheets also confirmed that the oxidation rate of the curved PAH substrate was faster than planar ones, especially during the initial stage of oxidation [200]. The underlying mechanism was further identified: Curved PAH substrate tends to form a larger number of sites with edge five-member rings that can potentially desorb to expel carbon into the gas phase (i.e., not due to actual oxidation reactions) [292]. On the other hand, as oxidation progresses, many five-member rings at armchair sites accumulated, due to oxyradical decomposition. These five-member rings then formed zig-zag edges, which tends to decrease soot reactivity [292, 350]. To summarize, the detailed work of Singh and Frenklach [292] confirmed that 
the effects of the five-member ring on soot oxidation depends on their position in the graphene substrate, emphasizing the importance of sterically-resolved simulations.

In addition to the reaction between oxygen and radical surface sites, the possibility of $\mathrm{O}_{2}$ reacting with saturated $\mathrm{C}_{\text {soot }}-\mathrm{H}$ sites was also investigated [200]. It was found that the possible reaction channel is for $\mathrm{O}_{2}$ to abstract the $\mathrm{H}$ radical, forming $\mathrm{HO}_{2}$. Nevertheless, the activation energy of such a reaction is high, making it less favorable than the oxidation at radical sites [200].

Soot fragmentation can be thought of as a reverse process of coalescence and agglomeration. Fragmentation can occur during thermal treatment of aggregated particles in an inert environment [371], but are more likely to happen during the oxidation processes. As discussed by Sirignano et al. [372], two types of fragmentation are possible: The first has to do with the fragmentation of large aggregates into smaller ones, or into isolated primary particles (i.e., aggregate fragmentation, reverse process of agglomeration); The second type is the fragmentation of primary particles into even smaller ones (i.e., particle fragmentation, reverse process of coalescence).

Aggregate fragmentation occurs when oxidative material are removed at the contact points linking primary particles; particle fragmentation is related to the internal structure of an individual particle [372]. Evidences for both types have been observed in different configurations such as two-stage burners [355-358], coflow [373], and counterflow diffusion flames [372]. Visual tracking of a particular single aggregate in an environmental TEM provided direct confirmation that the thickness of the bridge connecting primary particles is continuously reduced by oxidation before breakup, releasing two smaller aggregates [345]. These results show that fragmentation is an integral process of soot evolution and its accurate description is important if particle morphology, average sizes-and especially size distributions [374]-are to be accurately predicted for soot particles experiencing oxidation.

Due to the scope of the present review, oxidation of soot by $\mathrm{NOx}, \mathrm{H}_{2} \mathrm{O}$ and $\mathrm{CO}_{2}$ will not be discussed further; interested readers may find relevant information elsewhere [329]. It is, however, 
relevant to note, as Frenklach et al. mentioned in a recent publication [200], that soot oxidation chemistry is very rich and could be even more complicated than soot growth. As such, it is clear that more experimental and numerical work is needed for the development of predictive models of soot oxidation (and associated fragmentation) which will account for sterically-resolved surface reactions, effects of particle nanostructures, and direct coupling of the surface and gas phase environments.

\subsection{Models of soot formation}

With the above subsection providing physicochemical descriptions of soot formation processes, we intend to present an overview in this subsection on how these processes are numerically modeled. In fact, some modelling perspective has already been given, but here we will focus more on the numerical techniques used to deal with particle dynamics. Historical developments of soot models can be found in the review by Kennedy [120].

In many recent models for soot formation, soot inception was modelled as the physical collision of large PAHs such as pyrene [282, 375-377], or an ensemble of larger aromatics up to coronene [111, $140,378]$. In these studies, nucleation reactions were generally assumed to be irreversible with zero activation energy [198]. Recently, reversibility of the inception process was considered [288, 289, 379], and statistical mechanics were employed to determine the enthalpy and entropy of the PAH dimerization processes, through which equilibrium constants and reverse rate coefficients for soot nucleation reactions can be evaluated. The growth from gas-phase to nanoparticles was modeled as a purely chemical growth process in Refs. [136, 137, 217, 304, 380, 381]. The relevant model such as Atomistic Model for Particle Inception (AMPI) model [304, 380] and Stochastic Nanoparticle Simulator (SNAPS) $[136,137,217,381]$ were shown to correctly predict the order of magnitude of the nascent particle concentration. 
Once incepted, nascent soot particles will experience surface reactions and particle-particle interactions, and their sizes and morphology will change. For the model of these processes, a particle population balance equation needs to be solved, which can be written generally as:

$$
\frac{d N_{i}}{d t}=\frac{1}{2} \sum_{j=1}^{i-1} \beta(j, i-j) N_{j} N_{i-j}-\sum_{j=1}^{\infty} \beta(i, j) N_{i} N_{j}
$$

where $N_{\mathrm{i}}$ is the number density of particle of size $i . \beta(i, j)$ is the collision kernel between particles with sizes $i$ and $j$. The first two terms on the right-hand side are respectively the source and sink terms due to coagulation process. Note the specific form of the collision frequency depends on the coagulation regime (free-molecular, continuum, and transition), which is characterized by the Knudsen number, $K_{\mathrm{n}}=2 \lambda_{\mathrm{f}} / d$, where $\lambda_{\mathrm{f}}$ is the mean free path for the gas-phase and $d$ is the particle diameter. $\beta(i, j)$ is a non-liner function of the sizes of the colliding particles, and it is quite difficult to find a closed-form solution for Eq. 3. In this regard, several approximate numerical techniques such as Monte Carlo (MC) method, method of moments and discrete sectional method have been proposed.

Monte Carlo (MC) based models [382-384] could provide accurate results by tracking the evolution of number density through an assemble of particles governed by stochastic processes [382]. Nevertheless, these simulations are computationally demanding so that their applicability has so far been limited to simple configurations such as zero-dimensional reactors [385]. In contrast, the other deterministic models including method of moments and sectional method have been successfully applied to more challenging multi-dimensional environments.

For the method of moments (MoM), the detailed description of particle dynamics (i.e. Eq. 3) is reformulated in terms of the moments of particle size distribution function (PSDF). Specifically, the $r^{\text {th }}$ moment $M_{\mathrm{r}}$ is defined as [386]:

$$
M_{r}=\sum_{i=1}^{\infty} m_{i}^{r} N_{i}
$$

where $m_{\mathrm{i}}$ is the mass of particle of size class $i$. By taking the moment at both sides, Eq. 3 can be rewritten as [386]: 


$$
\begin{array}{cr}
\frac{d M_{0}}{d t}=-\frac{1}{2} \sum_{i=1}^{\infty} \sum_{j=1}^{\infty} \beta(i, j) N_{i} N_{j} & \text { Eq. } 5 \\
\frac{d M_{1}}{d t}=0 & \text { Eq. } 6 \\
\frac{d M_{r}}{d t}=\frac{1}{2} \sum_{k=1}^{r-1}\left(\begin{array}{l}
r \\
k
\end{array}\right)\left(\sum_{i=1}^{\infty} \sum_{j=1}^{\infty} m_{i}^{k} m_{j}^{r-k} \beta(i, j) N_{i} N_{j}\right) & \mathrm{r}=2,3, \ldots \text { Eq. } 7
\end{array}
$$

Numerical scheme such as the interpolative closure or variants of the quadrature method (see Ref. [375] and the references therein) is necessary to close the system of equations for the moments. Following the work of Guo et al. [387], the one-dimensional transport equations for the $r^{\text {th }}$ moment can be written as:

$$
\frac{\partial M_{r}}{\partial t}+\frac{\partial}{\partial x}\left(u M_{r}\right)=\frac{\partial}{\partial x}\left(\mathcal{D}_{p, 1} \frac{\partial M_{r-2 / 3}}{\partial x}\right)-\frac{\partial}{\partial x}\left(V_{T} M_{r}\right)+\dot{M}_{r}
$$

where $x$ denotes the Cartesian coordinate, $\rho$ and $u$ are the density and gas velocity, respectively. $V_{\mathrm{T}}$ is the thermophoretic velocity of soot particles, $\mathcal{D}_{p, 1}$ is the diffusion coefficient for the smallest soot particle. $M_{\mathrm{r}-2 / 3}$ is the fractional moments obtained by interpolation between integer moments. $\dot{M}_{r}$ is the source terms due to nucleation, coagulation, surface reaction (including oxidation), and PAH addition. Similar to the coagulation term, the other source terms are also needed to be rewritten in terms of soot moments[386], and they generally involve fractional moments.

In principle, a knowledge of all these moments is equivalent to a knowledge of PSDF itself [386]. However, in most cases the interested soot properties can be adequately represented by a finite number of moments (e.g., the first three or six moments). For example, the overall number of particles can be represented by the zeroth moment, $M_{0}$; the total volume of particles is the first moment $M_{1}$ (i.e. soot mass) divided by soot particle density; the average diameters of the particle ensemble can be derived from the ratio of for example, $M_{1} / M_{0}$. As a result, the method of moments could provide many expected soot properties, and in the meanwhile, keep the computational cost at a relatively low level. Therefore, it has been widely employed to compute soot particle dynamics of both laminar [122, 140, 198, 280, 375, 386, 388-391] and turbulent flames[392-394]. 
Discrete sectional method $[56,214,282,338,376,379,395-400]$ is another widely used method to approximate the population balance equation. In sectional method, the continuous size distribution was discretized by a finite number of sections (or bins). Each section has a representative particle size (or mass) and the mass/volume ratio of two consecutive sections is described by a constant spacing factor. It is obvious that the PSD can be resolved more accurately if more sections are used. In reality, the choice of section number depends on the tradeoff between the approximation accuracy and computational cost.

The solution for population balance equation with the sectional method is straightforward, since the prescribed sizes $i$ and $j$ directly correspond to representative particles for the sections. Similarly, the one-dimensional transport equation for the particle number density $\left(N_{\mathrm{i}}\right)$ in each section can be derived as:

$$
\rho \frac{\partial N_{i}}{\partial t}+\frac{\partial}{\partial x}\left(\rho u N_{i}\right)=\frac{\partial}{\partial x}\left(\rho \mathcal{D}_{\text {soot }, i} \frac{\partial N_{i}}{\partial x}\right)-\frac{\partial}{\partial x}\left(\rho N_{i} V_{T}\right)+\rho \dot{\omega}_{\text {soot }, i}
$$

where $\mathcal{D}_{\text {soot }, i}$ is the diffusion coefficient of soot particle in section $i$. Note the Brownian diffusion of soot particles is relatively weak and in some cases its effects on particle transport can be neglected [401]. $\dot{\omega}_{\text {soot }, i}$ is the soot source term for section $i$ including nucleation, coagulation, and heterogeneous soot surface processes. Incipient soot particles due to nucleation reside in the first few sections. Through coagulation or surface growth, particles in lower section can move to higher sections. On the other hand, particles in higher sections can move to lower sections by oxidation.

It is noted that Eq. 9 assumes that all soot particles are spherical (i.e., without considering the aggregate structures). This assumption was preferred in several soot models [56, 214, 378, 379, 397, $398]$ due partly to its low computation cost. If the soot aggregation was considered [282, 396, 402404], additional transport equation for the primary particle number density is required. In addition, additional assumptions are necessary. Frequently, it is assumed that all aggregates are comprised of equal-sized spherical primary particles, and they also have the same fractal dimension $D_{\mathrm{f}}$ of 1.8 , while smaller primary particles are assumed to be dense spheres with $D_{\mathrm{f}}$ of 3.0 . 
Kinetics describing the heterogeneous soot surface processes (i.e. surface growth, oxidation and condensation) are similar for both the method of moments and the sectional method, although the specific implementation is different. As mentioned previously, the soot surface growth model is mostly based on the HACA soot surface reaction scheme developed by Frenklach and coworkers $[198,280]$. The kinetics of the surface reactions are described using the concept of soot surface site

$\left(\mathrm{C}_{\text {soot}}-\mathrm{H}\right)$. The modeling of soot oxidation by $\mathrm{OH}$ is based on the kinetic theory and a widely-used value of this collision efficiency is 0.13 . Soot oxidation rate by $\mathrm{O}_{2}$ can be calculated based on the concept of soot surface site, similar to the modeling of HACA kinetics. PAH addition rate can be modelled based on the collision rate between large aromatics molecules (such as pyrene) and soot particles. Note that unlike chemical growth reactions, which require surface sites, PAH addition can be regarded as a physical process such that the rates are independent on the concentration of surface species. As not all collisions lead to condensation, a collision efficiency was generally implemented to represent the probability of a successful addition of the PAH onto the soot particle surface.

We finally pointed out the importance of more recently-proposed sterically-resolved particle surface reaction modelling, as discussed in Section 2.3.

\section{Experimental techniques for soot related measurements}

Experimental determination for the concentrations of soot related gas- and condensed-phase species are of central importance in soot mechanism studies. Experimentation not only provides the data to validate soot models but is also a source for new insight into soot formation. As a comprehensive understanding of soot-related diagnostic methods-including their advantages and limitations-is necessary for full appreciation of the experimental data, we provide in this section a brief overview of the most widely-used experimental techniques. 
3.1 Measurements of gas-phase species

\subsubsection{Gas chromatography}

Gas chromatography (GC) is a major technique for separating individual gaseous compounds from their complex mixtures. The most important part of a gas chromatograph is a specially coated long column through which a mixture is separated. Inside this column, gaseous compounds (mobile phase) interact with the inner coating of the column wall (stationary phase). These interactions are dependent on the physical properties of individual gaseous components; therefore, different compounds elute from the column after different retention times, leading to mixture separation. Additional sensors are required to detect and quantify the eluate, and those widely used for combustion products are the flame ionization detector (FID) for hydrocarbons, and thermal conductivity detector (TCD) for $\mathrm{H}_{2}$, $\mathrm{CO}, \mathrm{CO}_{2}, \mathrm{H}_{2} \mathrm{O}, \mathrm{N}_{2}$ and other non-combustible gases. In many cases, a mass spectrometer (MS) is also used as a highly effective detector, comprising a GC-MS system.

Figure 6 shows an example of a GC setup used by Gomez and coworkers [80] for the speciation of gas samples from counterflow flames. As can be seen, this particular setup adopts three parallel columns connected to different detectors. The TCD was used to quantify $\mathrm{N}_{2}$ and $\mathrm{O}_{2}$, which are present as large fractions of the sample. The FID was employed for the quantification of hydrocarbons as well as $\mathrm{CO}$ and $\mathrm{CO}_{2}$, with the help of an upstream methanizer. The identification of the species was achieved by checking the molecule-specific mass spectrum. 


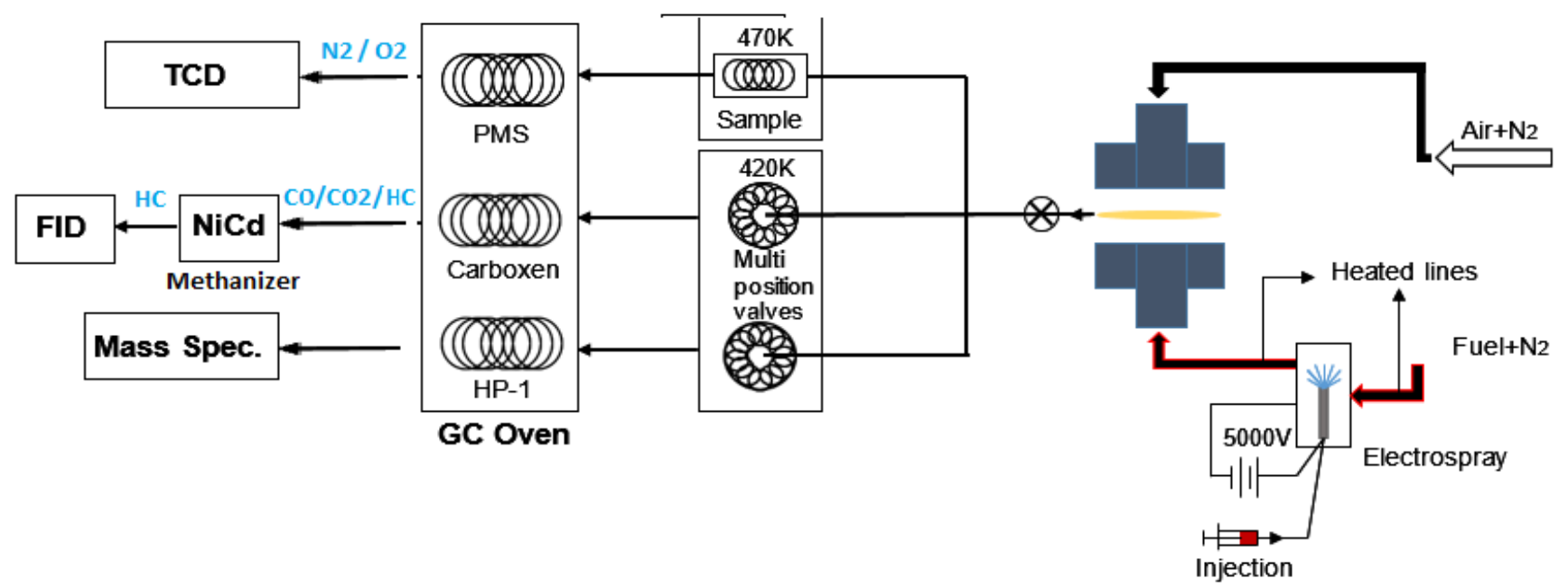

Figure 6. Schematic of GC setup for speciation of counterflow flames. Adapted from Jahangirian et al. [80] with permission of Elsevier.

A GC analysis requires probe sampling; therefore, it is an intrusive measurement. Probe disturbance is inevitable and needs to be considered, especially for small laboratory flames. The nozzle inner diameter of a typical microprobe used for product extractions is on the order of $100 \mu \mathrm{m}$ $[80,242,405,406]$. Although this size seems small, the presence of the probe can still cause the species spatial profile to shift up to several millimeters. In addition, special care must be given when measuring soot-laden flames since the small nozzle can potentially become clogged by particles. Another major drawback of GC analysis is that it may take up to several hours for all the species of interests to elute from the column, especially for large molecular species. This means that the GC method is an inherently off-line technique so that detection of short-lived radicals is impossible. As typically performed by Jahangirian et al. [80] and can be seen in Figure 6, gas samples, after being extracted from various locations of a flame, were stored in a set of sample loops to be analyzed overnight. If the species of interest include those with high boiling points (such as PAHs), the probe, the sample lines, and the storage loops must be heated to avoid condensation [407]. In such cases, it is necessary to determine whether thermal aging can cause variation in the species concentration. The 
condensation problem also put an additional limit on the maximum molecular weight that can be analyzed by GC.

\subsubsection{Mass spectrometry}

A mass spectrometer (MS) identifies different chemical species by ionizing neutral molecules and measuring the mass-to-charge ratios. In addition to use as a detector in a GC-MS system, a MS can also be used separately for combustion speciation, provided suitable sampling and ionization techniques are employed. Perhaps the most popular sample introduction method is through molecularbeam (MB) $[408,409]$; its application in combustion diagnostics has been periodically reviewed by Biordi [410], Westmoreland et al. [411], Hansen et al. [172], and Qi [412]. Figure 7 shows an experimental setup used by Skeen et al. [413] for the chemical speciation of counterflow diffusion flames using molecular beam sampling. As can be seen, the apparatus mainly consists of a counterflow burner enclosed in a low-pressure chamber, a double-stage pumping flame-sampling system, and a time-of-flight mass spectrometer. An important advantage of the MBMS technique is its ability to preserve radicals in a molecular beam for further MS detection. Nevertheless, it is still noteworthy to point out that MB sampling, as an intrusive diagnostic method, is not used directly to sample the counterflow flames, but the material extracted from the flames via a quartz millimetersized probe. 


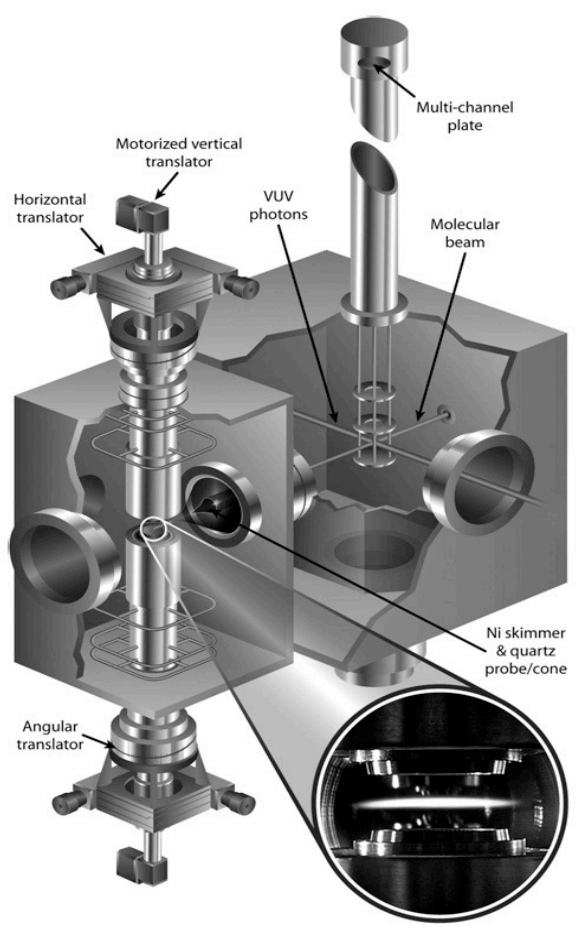

Figure 7: Schematic of counterflow burner coupled to a time-of-flight mass spectrometer with molecular beam sampling and VUV photoionization. Reprinted from Skeen et al. [413] with permission of Elsevier.

After the formation of a molecular beam, neutral atoms or molecules must be ionized before they can be sorted and identified based on their mass-to-charge ratios. Various ionization methods have been developed, among which electron ionization (EI), laser-based photoionization, and synchrotron VUV photoionization are the most widely-used in combustion research. EI is based on the bombardment of high energy electrons to molecules in producing ions. EI cross-sections are a strong function of electron energy, with rather low values near the ionization threshold for most species. Therefore, for strong signals the electron energy is frequently set at a level much higher than the typical ionization energy threshold for combustion species (around $70 \mathrm{eV}$, as the maximum ionization cross-sections occur at this energy level). Unfortunately, these energetic electrons can cause significant fragmentation of the parent molecules. If the sample is a single compound, the fragmentation pattern produced provides a signature of its molecular structure and thus is helpful for the structural determination of unknown species. However, if the sample is a complex mixture, the 
fragmentation may cause significant difficulties in deconvoluting individual species, if not making the process completely impossible. In this regard, MS with EI is thought to be more suitable for use as a detector for GC-MS systems where the mixtures are separated before entering MS.

To minimize or avoid fragmentation, ionizing sources with wide tunability (in the range of ionization energy of combustion relevant species, typically $7-16 \mathrm{eV}$ ) and high energy resolution are required. Synchrotron radiation in the VUV regime has been proven to be an ideal ionization source [412]. The photon energy can easily be tuned to just above the threshold ionization energy of most combustion intermediates so that the neutral molecules/atoms/radicals can be ionized (single-photon ionization) without significant fragmentation, resulting in much cleaner mass spectra for easy analysis. Furthermore, by recording the mass spectra of a certain species at different photon energies, a PIE spectrum can be obtained. The information about ionization energy contained in a PIE spectrum allows unknown species to be identified by comparison against values in the literature or obtained from quantum computation. Since most isomers have different ionization energy, a PIE provides an effective way to distinguish isomers. Prominent examples are the separation of fulvene and benzene $\left(\mathrm{C}_{6} \mathrm{H}_{6}\right)$, allene and propyne $\left(\mathrm{C}_{3} \mathrm{H}_{4}\right)$ [171], and ethenol and acetaldehyde $\left(\mathrm{C}_{2} \mathrm{H}_{4} \mathrm{O}\right)$ [414], among others.

With all its advantages, synchrotron radiation is only accessible in a limited number of locations (mostly large national laboratories) across the globe. Consequently, beam time dedicated to combustion speciation is extremely limited. As an alternative, VUV lasers can also be used for singlephoton photoionization, although these laser-based methods suffer from two major drawbacks: 1) The photon energy is either fixed or inconvenient to tune and 2) Typical maximum photon energy is not enough to ionize species with high ionization energies. Bermudez and Pfefferle [415] frequency tripled the third harmonic of a Nd-YAG laser in a xeon cell to produce a VUV beam at $118 \mathrm{~nm}$ (i.e., photon energy of $10.5 \mathrm{eV}$ ), which was subsequently used to ionize most $\mathrm{C}_{3}$ and larger hydrocarbon species (except $\mathrm{C}_{3} \mathrm{H}_{8}$ and $\mathrm{n}-\mathrm{C}_{4} \mathrm{H}_{10}$ ) for MS analysis [416]. However, major combustion products, and most $\mathrm{C}_{2}$ species, have ionization energy above $10.5 \mathrm{eV}$ and thus cannot be photoionized. To achieve 
tunability, a four wave sum-difference frequency generation in a krypton cell can be used, which typically requires two dye lasers and has been shown to produce broadly tunable VUV radiation with photon energy between 6.2 and $10.3 \mathrm{eV}$ [417]. This technique has been successfully used by Qi and McIlroy [418] and Werner and Cool $[419,420]$ for combustion speciation.

In addition to single-photon ionization, resonance-enhanced multi-photon ionization (REMPI) has also been used in MBMS measurements [421-423]. REMPI involves the process of resonant multiphoton (typically 1 or 2) absorption by a molecule or atom to reach an intermediate Rydberg state, followed by additional photon absorption that leads to ionization. The signature vibronic transitions of individual species, together with the photon energy tunability of dye lasers, provides species selectivity. Even in cases where there is an absorption energy band overlap so that more than one species can be ionized with the same laser wavelength, the produced ion mass may be different; thus the combination of MS and REMPI offers the possibility for two-dimensional analysis. When the excitation photons and the ionizing photon are of different energies, two-color REMPI provides an additional method if the conventional, one-color REMPI technique is insufficient for species selectivity. In fact, REMPI-MS is both mass-selective and isomer-selective, due to the fact that isomers with different molecular structures have different spectroscopic features. It is believed to be especially suited for selectively ionizing and measuring aromatic species [424-428]. It is, however, worthwhile to mention that, ideally, the samples should be supersonically jet-cooled to an ultra-low rotational temperature (i.e., tens of Kelvins) before being photon-ionized, in order to reduce the broadening of energy levels which are detrimental to REMPI energy selectivity. Fortunately, this can be achieved by molecular beam sampling, although care must be taken when sampling high temperature combustion products at low pressures.

A notable advantage of REMPI, as compared to tunable laser-based VUV, is that the beam can be more easily obtained via a single dye-laser in the comfortable UV regime. The drawbacks of REMPIMS are: 1) Many necessary spectroscopic data (i.e., energy bands, excitation cross-sections, 
ionization cross-sections, and etc.) are not readily available [123]; 2) Significant experimental time is required as the photon energy (i.e., the laser wavelength) must be changed [123], and related calibration must be performed (ideally) for each species of interest; and 3) Its application is typically limited to small and medium-sized aromatic species [412]. To summarize, of the above three different ionization methods, EI is universal but not selective, REMPI is selective but not universal, and tunable VUV is both universal and selective. It is important to mention, however, that REMPI combined with MBMS is probably the method of choice [127] for quantitative PAH measurements, due to the sensitivity enhancement from resonance, which is especially important for species with ultra-low concentrations such as PAHs.

\subsubsection{Optically-based in-situ methods}

Both GC and MS techniques are intrusive techniques, thus they share the disadvantages of flame perturbations. The presence of the probe may affect the temperature/concentrations profile as well as the flow field [429]. On the other hand, laser-based optical methods can provide an in-situ determination of species concentrations from unperturbed (in most cases) flames.

Species-selective laser-based combustion diagnostic techniques primarily include laser absorption spectroscopy (LAS) [430, 431], laser-induced fluorescence (LIF) [432], and Raman scattering. Excellent books and reviews for these methods are available in the literature [430, 431, 433]; here only a brief introduction is offered.

A molecule with dipole moment can absorb photons that have energy resonant with the spacing between two of its different ro-vibrational states, which are the signatures of individual species (thus species-selective). The extent of absorption is proportional to the number concentrations of the absorbing species, through the Beer-Lambert law, $I=I_{0} \times \exp \left(-\eta_{\lambda} L\right)$, where $I$ and $I_{0}$ are the transmitted and incident beam intensities, respectively; $\eta_{\lambda}$ is the spectral absorption coefficients and, $L$ is the path length. For a spectrally-isolated transition, $\eta_{\lambda}$ can be written as: 


$$
\eta_{\lambda}=s(T) \times \varphi(\lambda) \times P_{\mathrm{i}}
$$

where $s(T)$ is the temperature-dependent transition line strength, $\varphi(\lambda)$ is the line shape function depending on thermodynamic conditions, and $P_{\mathrm{i}}$ is the partial pressure (thus mole fraction) of the probed species. Detailed expressions for $s(T)$ and $\varphi(\lambda)$ can be found in Ref. [434]. For large molecular species that do not present a spectrally-resolved rotational transition, the overall absorption crosssection $\left(\sigma_{\text {abs }}\right)$ can be used:

$$
\eta_{\lambda}=\sigma_{\mathrm{abs}} \times N_{\mathrm{i}}
$$

where $N_{\mathrm{i}}$ is the molecule number concentration. Although Eq. 11 appears to be simpler, $\sigma_{\text {abs }}$ must be experimentally calibrated for a specific gas under specific thermodynamic conditions, and an extrapolative extension to experimentally uncovered conditions is very difficult [430].

LAS is mostly conducted in the infrared spectrum since ro-vibrational transitions typically involve energy differences corresponding to photons in the near-mid infrared regime. Since each species has specific signature transitions, tunable diode laser source is generally the workhorse for LAS, providing wavelength tunability to be resonant with such transitions, hence the term tunable diode laser absorption spectroscopy (TDLAS). LAS is especially suitable for quantitative determination of species mole fractions in 0D shock tubes, 1D laminar flat premixed flames and axisymmetric 2D coflow and counterflow flames. Under such conditions, the local absorption coefficients $\left(\eta_{\lambda}\right)$ along the line-of-sight are either uniform or can be obtained through simple tomographic inversion. For unsteady multi-dimensional conditions which are predominant in practical combustion devices, the line-of-sight feature of LAS becomes a major limitation for its application.

In combustion environments where many intermediates coexist, the species to be quantified typically needs to have signature transitions (at high temperatures) that are interference-free from other species. Large molecules tend to have very complex and broad absorption spectra and will therefore almost always interfere with other species. As a result, quantifiable species quantification with LAS is most frequently done for small molecules or radicals such as $\mathrm{CO}, \mathrm{CO}_{2}, \mathrm{H}_{2} \mathrm{O}, \mathrm{CH}_{3}, \mathrm{CH}_{4}$, 
$\mathrm{C}_{2} \mathrm{H}_{2}$, and $\mathrm{C}_{2} \mathrm{H}_{4}$. Nevertheless, if interference can be suitably taken care of or if qualitative monitoring is targeted, measurements using LAS can be extended to practical combustion conditions for larger molecules such as n-heptane, iso-octane, and methyl-cyclohexane [435].

Another concern for TDLAS is the lower limit for detection. If the absorbance level is too low (either due to the low concentration of probed species or its low line strength), light absorption could be too weak, which may adversely affect the accuracy of measurements. In this regard, technical approaches are available to increase the path length, or cavity ringdown spectroscopy (CRDS) can be applied, which is based on the idea to reflect the laser beam back and forth inside the cavity bounded by two highly reflecting mirrors [436]. Typical mid-IR laser sources are tunable only in a relatively narrow wavelength range. Therefore, multiple lasers are required if more than one species specific need to be measured. We quote recent studies using TDLAS to measure $\mathrm{C}_{2} \mathrm{H}_{2}$ concentration formed during the pyrolysis of ethylene and benzene in a shock tube [39], and to measure temperature via $\mathrm{CO}_{2}$ absorption in sooting ethylene coflow diffusion flames [437] to demonstrate the potential of this method in quantifying important soot precursors as well as temperature under sooting conditions.

Laser-induced fluorescence (LIF) is a process in which the target molecule/radical is excited to a higher energy state after absorbing one or more (typically two) photons, and subsequently returned to the ground state by spontaneous emissions of photons (i.e., fluorescence). Qualitatively, species selectivity can be obtained by tuning the laser wavelength to be resonant with the target species. Quantitatively, the fluorescence intensity is proportional to the number concentrations of the probing species. A notable advantage of LIF as compared to LAS is spatial resolution: LIF is a point-based (as opposed to line-of-sight based) measurement with signals typically detected perpendicular to the incident laser beam. In addition, LIF can be configured to measure species concentration distribution in a 2D plane (planar LIF), by manipulating the excitation laser beam to form a light sheet and using an intensified charge-coupled device (ICCD) camera as a fluorescence signal detector. 
Unlike LAS, LIF typically involves electronic transitions of target species so that lasers in the UVVis spectrum are used as sources of excitation (electronic state transitions have energy differences corresponding to photons in the UV-Vis spectrum). This is due partly to the relatively large Einstein A coefficients of electronic transitions and partly to the availability of high-powered UV and visible lasers. In combustion studies, $\mathrm{OH}, \mathrm{CH}, \mathrm{H}$ radicals and $\mathrm{NO}$ and $\mathrm{CO}$ molecules are frequently measured with LIF. Note, however, due to the uncertainties in fluorescence quenching processes and the fluctuations of laser energies, quantitative determination can be challenging even for these small species. On the other hand, LIF is especially suitable for qualitatively tracking the spatial distribution and temporal evolution of flame front (as marked by $\mathrm{CH}$ or $\mathrm{OH}$ ). By seeding fluorescent tracers (i.e., acetone), LIF is also frequently used to visualize the mixing process of two streams.

In addition, LIF is used to measure PAH concentration in a qualitative manner. PAHs have somewhat complicated molecular structures and thus have fluorescence with broad and complex spectrum (further complicated because both temperature and surrounding atmosphere would considerably alter the spectra). In flame environments, the detected signals always represent the fluorescence of a mixture of PAH species, making quantitative deconvolution highly challenging, if not entirely impossible. Nevertheless, it has been shown that larger PAHs are better represented by LIF signals detected at longer wavelengths [438-440] so that a spectrally-resolved LIF signal can be used to qualitatively differentiate between small and large PAHs. The signal intensity can also be used to qualitatively evaluate the concentrations of these different classes of PAHs. This method is particularly useful in evaluating fuel effects on $\mathrm{PAH}$ formation by comparing the relative concentrations of PAHs as represented by LIF signal intensity among flames with different fuels or fuel additives [441-447]. Nevertheless, it is important to note that PAH LIF signal can be highly dependent on temperature [448]. As a result, quantitative comparisons of PAH LIF signals can be performed only when the temperature is the same, and care should be taken when evaluating LIF signals measured in areas with significantly different local temperatures. Furthermore, as mentioned 
above, many different PAH species can coexist in flame conditions. The fluorescence spectra of these PAH species are not only complex and broad, they can also significantly overlap with each other. Collisional quenching with surrounding gases serves as additional source of complications for quantitative PAH LIF analysis [449].

Nevertheless, there is recent progress towards quantitative measurement of PAH, using LIF. The new technique-called jet-cooled LIF-is based on the extraction of flame-generated species through a microprobe and subsequent expansion via a supersonic jet, before being analyzed using LIF in an analysis chamber $[55,450,451]$. The supersonic expansion reduced the temperature and also the possibility of an otherwise large number of vibronic transitions, making the fluorescence spectra more structured and amenable to selective detection. Furthermore, the LIF quenching effect can also be reduced, making quantification easier. Although this method requires probe sampling and thus is not non-intrusive, it provides an important alternative for quantitative PAH measurement in flames. Nevertheless, to date, the jet-cooled LIF has been successfully used only to measure a couple of PAH species and further efforts are required to extend its range of application and to quantify measurement uncertainties.

LIF can also be used to detect flame-generated nanoparticles which exhibit molecular characteristics [51, 290, 452, 453]. Differentiation between particles and PAH molecules can be obtained by time-resolved fluorescence polarization anisotropy [454-456].

More recently, infrared LIF (IR-LIF) has attracted interests since it can be used to measure important molecules such as $\mathrm{CO}_{2}, \mathrm{CO}$ and $\mathrm{H}_{2} \mathrm{O}$, which are not accessible by conventional $\mathrm{UV}-\mathrm{Vis}$ LIF techniques $[457,458]$. The application of this technique in harsh combustion environments is expected to be promising, especially considering that it can also provide 2-D temperature distribution using a two-color method [457] (conventional two-color OH PLIF for temperature measurements is restricted to regions where $\mathrm{OH}$ are abundant). We finally quote a recent study using femtosecond 
two-photon laser-induced fluorescence (fs-TPLIF) to measure $\mathrm{H}$ atom in sooting premixed flames [459] to demonstrate the applicability of LIF in sooting flames.

Another species-selective technique frequently employed in combustion research is Raman scattering, which is based on inelastic interactions (as opposed to the elastic Rayleigh scattering) between incident photons and target molecules. The scattered light has a species-dependent fingerprint frequency shift relative to the incident light (Raman shift), which can be used to identify and quantify species. Spontaneous Raman scattering is suitable for point measurements of major combustion species such as $\mathrm{O}_{2}, \mathrm{CO}, \mathrm{CO}_{2}$ and $\mathrm{H}_{2} \mathrm{O}$. An important advantage of spontaneous Raman scattering is its ability to measure multiple species simultaneously [460-462]; while an important disadvantage is its very weak signal. In practice, high-energy pulsed laser beam is focused into a measurement point and the resulting Raman scattering signal is spectrally-resolved with a spectrograph before being recorded with an intensified camera. One major drawback of Raman scattering is the costly and bulky instruments (high-resolution spectrometer, high-energy laser and intensified detectors) it requires, which limit its application largely to laboratory conditions. Note, the Raman scattering signal intensity can be enhanced by tuning the incident photon energy resonant with a specific species of interests (i.e., resonance Raman scattering) [463].

Both the LIF and Raman scattering techniques discussed above are incoherent methods and the signals leave the sample in all directions, covering the whole $4 \pi$ solid angle, while the collection of the scattered signal can only be achieved over a relatively small solid angle. This reduced signal collection efficiency can be overcome by using coherent techniques in which the signal becomes beam-like and can be effectively directed to a detector. A thorough discussion of four-wave mixing techniques, among which the widely-used coherent anti-stokes Raman spectroscopy (CARS) is a particular configuration, was provided by Kiefer and Ewart [433]. Recent studies using CARS to measure temperature in sooting coflow diffusion flames $[464,465]$ are quoted here to demonstrate its applicability in sooting environments. 


\subsection{Soot measurements}

Soot loading in a flame is typically represented by soot volume fraction (SVF, or $f_{\mathrm{v}}$ ), so its experimental determination is central to soot research. Therefore, it is worthwhile to provide a discussion here on various diagnostic techniques developed for quantitative SVF measurements.

\subsubsection{Light extinction}

Soot particles are known to absorb light in a broad spectral range from UV to IR, so that the intensity of any light beam (visible or near IR) passing through a soot-laden flame is expected to be attenuated. The extent of such intensity reduction can be correlated to the volumetric loading of soot particles. In typical light extinction experiments, the intensity of a probe beam with a fixed wavelength $(\lambda)$ is measured both before $\left(I_{\lambda, 0}\right)$ and after $\left(I_{\lambda}\right)$ passing the flame, and their ratio is defined as the light transmissivity $\left(\tau_{\lambda}\right)$. The local extinction coefficient $K_{\text {ext, },}$ can then be obtained through the Beer-Lambert law as:

$$
\tau_{\lambda}=I / I_{0}=\exp \left(-\int_{0}^{L} K_{\text {ext, } \lambda} d s\right)
$$

where $L$ is the length of light path. For a homogeneous system in which $K_{\text {ext, } \lambda}$ is a constant along the optical path, Eq. 12 can be reduced to a simpler form:

$$
K_{\mathrm{ext}, \lambda}=-\ln \left(\tau_{\lambda}\right) / L=-\ln \left(I / I_{0}\right) / L
$$

For axisymmetric cases such as counterflow or coflow diffusion flames, an inversion algorithm [466, 467] can be used to derive local $K_{\text {ext }, \lambda}$ from multiple realizations of line-of-sight measurements.

The inference of SVF from measured local extinction coefficients requires fundamental knowledge of soot-light interactions. The complex soot morphology makes it computationally demanding to use exact theories to analyze such interactions. Consequently, researchers frequently use the RayleighDebye-Gans-fractal aggregate (RDG-FA) theory as an approximation of the otherwise intensive analysis. According to the RDG-FA theory, the local extinction coefficient can be written as: 


$$
K_{\mathrm{ext}, \lambda}=N_{\mathrm{pp}} \pi^{2} D_{\mathrm{pp}}{ }^{3} E\left(\widetilde{m}_{\lambda}\right) / \lambda
$$

where $N_{\mathrm{pp}}$ and $D_{\mathrm{pp}}$ are the number density and the diameter of primary particles, respectively. $\tilde{m}_{\lambda}$ is the wavelength $(\lambda)$-dependent soot complex refractive index and $E\left(\widetilde{m}_{\lambda}\right)$ is the light absorption function, which is defined as:

$$
E\left(\widetilde{m}_{\lambda}\right)=-\operatorname{Im}\left(\frac{\widetilde{m}_{\lambda}^{2}-1}{\widetilde{m}_{\lambda}^{2}+2}\right)
$$

Since in the RDG-FA theory, soot particles are treated as fractal aggregates of mono-dispersed spherical particles in point contact, Eq. 14 further shows the total volume of the soot aggregates (i.e., soot volume fraction, $f_{\mathrm{v}}$ ) to be:

$$
f_{\mathrm{v}}=\frac{\lambda K_{\mathrm{ext}, \lambda}}{6 \pi E\left(\widetilde{m}_{\lambda}\right)}
$$

Note that in writing Eq. 14, it was assumed that light extinction is solely due to soot absorption. In reality, however, both particle scattering and light absorption by gas-phase species (such as PAHs) may contribute to intensity attenuation of the transmitted light. The ratio of scattering coefficient to absorption coefficient can be determined, provided the morphology of particles is known (see, e.g. Ref. [468]). Nevertheless, when the optical sizes of soot aggregates are significantly smaller than the laser wavelength ( $2 \pi R_{\mathrm{g}} / \lambda<0.3$, where $R_{\mathrm{g}}$ is the radius of gyration of agglomerate), light scattering becomes negligible compared to absorption $[469,470]$. The absorption by a gas-phase species can be estimated by running the HITRAN code for typical combustion products. Simonsson et al. [471] found that for a rich-premixed flame, molecular absorption in the near-infrared regime (700-1064nm, typically used for soot measurement) from major combustion products, is dominated by $\mathrm{H}_{2} \mathrm{O}$ molecules at $978.6 \mathrm{~nm}$. However, the absorption strength was rather small and its contribution to soot light extinction was concluded to be marginal [471]. This is especially true in practical cases where it is rare for the light wavelength used for soot measurement to be coincident with the narrow spectral line resonant for molecular absorption. 
On the other hand, PAHs, with their complex molecular structures, are known to absorb significantly in the UV-Vis spectral range [472]. Since PAHs are precursors to soot, flames with high soot loadings are also featured with high concentrations of PAHs, so that PAH interference is expected to become worse for more sooting flames. If the wavelength of the probe light lies in such a UV-Vis spectrum, the interference of soot extinction from PAH absorption must be accounted for. The simplest, and perhaps the most effective remedy, is to perform soot extinction measurements with light wavelength in the near-infrared regime (i.e., $>700 \mathrm{~nm}$ ), for which PAH absorption is minimal [473]. The employment of a near-infrared beam may have additional benefits as the absorption ratio for soot aggregates is predicted to decrease with increasing $\lambda[127]$. However, it is worthwhile to point out that previously many studies in the literature used light in the visible spectrum for soot extinction measurement. Thus, this fact should be considered when interpreting data in the literature.

A major source of uncertainty in light extinction measurement is the quantitative variations of the soot complex refractive index $\widetilde{m}_{\lambda}$ (and thus $E\left(\widetilde{m}_{\lambda}\right)$ ) on wavelength, and on the physical/chemical properties of soot produced from different sources. The spectral dependence of $E\left(\widetilde{m}_{\lambda}\right)$ for mature soot from coflow ethylene diffusion flames has been studied using laser-induced incandescence (LII, discussed later) with both 532 and $1064 \mathrm{~nm}$ beams. It was determined that the absorption crosssections have a $\lambda^{-0.83}$ dependence [474]. Cleon et al. [475] determined $E\left(\widetilde{m}_{\lambda}\right)$ along a rich premixed burner-stabilized methane flame and found that the ratio of $E\left(\widetilde{m}_{532 n m}\right) / E\left(\widetilde{m}_{1064 n m}\right)$ decreased significantly with the height above the burner (HAB) in the nascent soot region, but it tended to level off at higher HAB. This indicates that soot at different flame locations have different optical properties. Nevertheless, the optical properties of mature soot appear to have a more consistent value [127].

In many cases, a narrow laser beam is sent through a soot-laden flame and a single-point photodiode is used to detect the transmitted beam intensity. For a one-dimensional soot field (in a 
burner-stabilized flat premixed flame, or planar counterflow diffusion flames), the spatial resolution of measurement is then determined primarily by the beam size. The spatial variation of the soot field is obtained by passing the beam through different locations of the flame, realized by either moving the flame relative to a fixed beam $[140,476]$, or moving the complete optical setup relative to a fixed flame burner [69]. A major disadvantage of such line-by-line measurements is that it may take a longer time to scan the entire flame, thus it is not practical to measure soot fields that are time dependent. Alternatively, a 2D collimated beam that covers the entire flame area (projection along the direction of the beam) can be employed, with a camera serving as the device for light intensity detection $[477,478]$. In such a configuration, every pixel of the camera records the intensity of a light ray transmitted through a different location of the flame. Hence, a complete map can be obtained at once, allowing for instant measurements of time-varying soot fields, for example, in flickering coflow diffusion flame [479]. Note that beam steering, which is caused by the lensing effect of flame gases, must be minimal or its influence should be accounted for in extinction measurements, especially in cases where a large temperature gradient (therefore a large density gradient) exists. Examples of such cases include counterflow diffusion flames and high-pressure coflow flames [477].

\subsubsection{Spectral soot emission}

The spectral emissions of flame-heated soot particles provide information on both soot temperature and volume fractions. In axisymmetric flames, soot radiation transmitted along a line-of-sight can be detected and spectrally resolved using a spectrometer. A typical optical setup for such spectral soot emission (SSE) measurement is schematically shown in Figure 8. The detected light intensity can be described as [480, 481]:

$$
I_{\lambda}\left(y_{0}\right)=\int_{-\infty}^{\infty} \eta_{\lambda}\left(x, y_{0}\right) B_{\lambda}\left[T\left(x, y_{0}\right)\right] \exp \left(-\int_{x}^{\infty} K_{\text {ext }, \lambda}\left(x^{\prime}, y_{0}\right) \mathrm{d} x^{\prime}\right) \mathrm{d} x \quad \text { Eq. } 17
$$

where $y_{0}$ denotes the distance between the line-of-sight chord and the symmetry plane of a flame/burner; $x$ is the distance along the chord under consideration, with 0 defined at the symmetry 
point; $\eta_{\lambda}$ is the local soot absorption coefficient, and is equal to the soot extinction coefficient $K_{\text {ext }, \lambda}$ under the assumption of negligible scattering [482], so that it is related to SVF through Eq. 16. $B_{\lambda}$ is the Planck function of wavelength $\lambda$ and temperature $T$ and is expressed as:

$$
B_{\lambda}(T)=\frac{2 \pi h c^{2}}{\lambda^{5}} \frac{1}{\exp (h c / \lambda k T)-1} \approx \frac{2 \pi h c^{2}}{\lambda^{5}} \exp \left[-\frac{h c}{\lambda k T}\right] \quad \text { Eq. } 18
$$

where $h, c$, and $k$ are the Planck constant, light speed, and Boltzmann constant, respectively. Note that the Wien approximation to the Planck's law (rather accurate in the visible to near infrared spectrum) is invoked in writing in the latter half of Eq. 18. In Eq. 17, the term of $\eta_{\lambda} B_{\lambda}$ (i.e., $K_{\text {ext, } \lambda} B_{\lambda}$ ) represents the local spectral emission, and the exponential term takes the form of the Beer-Lambert law to describe the attenuation of such emission by self-absorption along the line-of-sight.

(a)
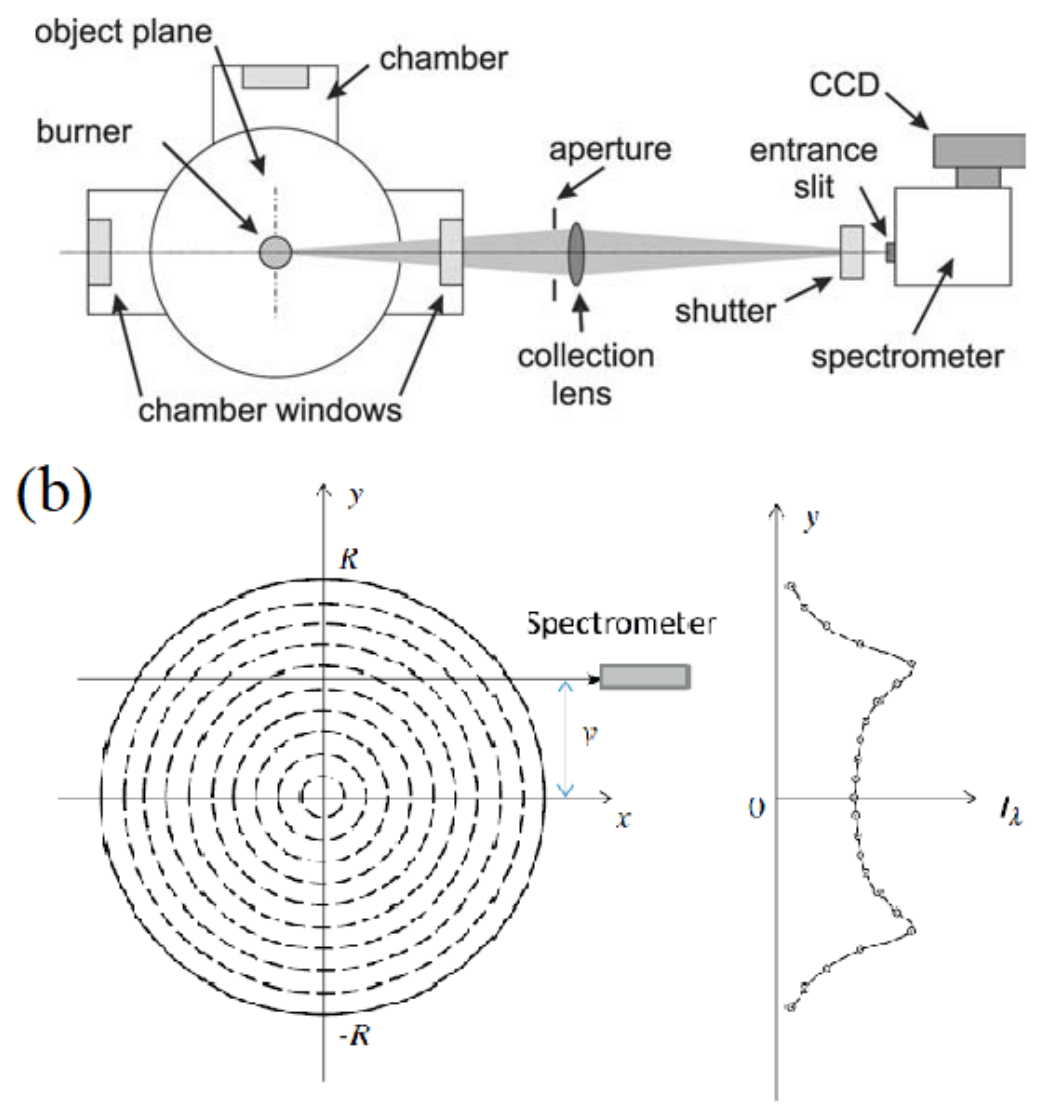

Figure 8. Typical optical setup for spectral emission-based soot temperature and volume fraction measurements (a), reprinted from Thomson et al. [483] with permission of Elsevier and schematics 
of line-of-sight emission intensities $\left(I_{\lambda}\right)$ for axisymmetric flames (b), reprinted from Liu et al. [484] with permission of Elsevier.

Through horizontal translation of the flame relative to the detection system, line-of-sight measurements can be performed at different $y_{0}$, and a subsequent tomographic inversion will provide local values of $K_{\text {ext, } \lambda}(r) B_{\lambda}(r)$ [484]. Substituting Eq. 14 into Eq. 18, taking the logarithm on both sides, and considering $K_{\text {ext, } \lambda}(r) B_{\lambda}(r)$ is now known at all radial positions $r$ lead to [484]:

$$
\ln \left[\frac{E\left(\widetilde{m}_{\lambda}\right)}{K_{\text {ext, }, \lambda}(r) B_{\lambda}(r) \lambda^{6}}\right]=\frac{h c}{\lambda k T(r)}-\ln \left[12 f_{v}(r) \pi^{2} h c^{2}\right]
$$

As seen, the left side of Eq. 19 has a linear relationship with $1 / \lambda$ and the slope, $h c / k T$, is solely determined by temperature. In practical applications, $h c / k T$ can be obtained by a curve fitting using the method of least mean squares. Once the temperature is known, Eq. 19 can be used again to provide $f_{\mathrm{v}}$.

One practical challenge in this spectral soot emission technique is the accurate determination of the local values of $\eta_{\lambda}$, or equivalently $K_{\text {ext, } \lambda}$, with the assumption of zero scattering. First, the detection system must have a large depth of field to allow every emitter along the line-of-sight to be properly focused into the spectrometer. This was shown to be achievable using an imaging lens with a sufficiently large f-number [480] (for example, f/48 was used in recent studies [485, 486]). Second, the self-absorption of soot emission must be accounted for [480] because: 1) Emissions from different emitters along the line-of-sight will be attenuated unequally due to the different path lengths toward the detector, causing the axisymmetric assumption needed for Abel-like tomographic inversion to break down and 2) Self-absorption is also dependent on $\lambda$ and as such it complicates the soot temperature measurement by shifting the slope, as discussed in Eq. 19. In view of these issues, Snelling et al. [480] proposed an iterative method to correct for self-absorption, and Liu et al. [484] concluded that when the maximum flame optical thickness exceeds 0.3 such correction becomes particularly important. 
Also suggested by Eq. 19, the emissions theoretically need to be detected at only two wavelengths

to find the flame temperature and $f_{\mathrm{v}}$, considering two points in the $\left(\frac{E\left(\widetilde{m}_{\lambda}\right)}{K_{\text {ext, } \lambda}(r) B_{\lambda}(r) \lambda^{6}}, 1 / \lambda\right)$ plane can already determine the line whose slope is $h c / k T$. The experimental technique based on this fact is known as two-color pyrometry and it was also used extensively in soot measurements. Although the measurement uncertainty can be larger than its multi-wavelength counterpart, a spectrometer is no longer needed and 2D measurements are possible with a color filtered CCD camera as a detector [487, 488].

This spectral soot emission (SSE) technique has been applied successfully in many soot studies, especially for coflow diffusion flames at high pressures (see Karatas and Gülder [107] and references therein). A notable advantage of SSE is that no additional illuminating source is required, and for high-pressure applications, optical access is needed only from one side of a high-pressure chamber, simplifying the design of the pressure chamber [107]. It is noted, however, that SSE was employed for soot studies in counterflow diffusion flames only very recently by Gleason et al. [489, 490]. By contrasting the soot temperature as determined by SSE against the well-established temperature from the literature, the authors were also able to obtain information on the dispersion exponent, a soot optical property related to its hydrogen content.

\subsubsection{Laser-induced incandescence}

Laser-induced incandescence (LII) refers to the quasi-blackbody radiation of soot particles after being heated up to about $4000 \mathrm{~K}$ by high-energy laser beams. Through analysis of such radiation, many properties of soot ensemble can be derived, such as SVF and soot primary particle size. Dedicated reviews on the LII technique for soot diagnostic exist in the literature [126-128, 491], and a brief overview of the basics of this promising method is given here. 
The usefulness of LII stems from the fact that SVF is reasonably proportional to LII signal intensity (ILI) $[492,493]$. Melton [492] has shown that, for soot ensembles with primary particle sizes smaller than the incident laser wavelength, the LII signal intensity can be written approximately as:

$$
I_{L I I}=\mathrm{C}_{1} \int_{0}^{\infty} N P(D) D^{n} d D, \text { with } n=3+154 / \lambda_{\mathrm{det}} \quad \text { Eq. } 20
$$

where $N$ is the total particle number density, $P(D)$ is the normalized probability density for particle with diameter $D$, and $\lambda_{\text {det }}$ is the detection wavelength in nm unit. As can be seen, for larger detection wavelengths, the exponent $n$ approaches three and $I_{\text {LII }}$ becomes linearly proportional to SVF.

It is noteworthy that Eq. 20 was obtained in the limit of high laser power. Whether this limit is achieved in practice can be tested by plotting $I_{\text {LII }}$ as a function of laser pulse energy. Typically, $I_{\text {LII }}$ initially increases, reaching a plateau regime (saturation), then begins to decrease again (due to soot evaporation) with progressive increases in laser energy [494]. Experiments are typically conducted in the plateau regime so that the signals are not affected by the pulse-to-pulse energy variations of the laser beam. Due to potential interference from PAH fluorescence, it is generally recommended that infrared light be used as the excitation source [472], although a recent work [459] showed that by delayed detection, the SVF measured using UV $(266 \mathrm{~nm})$ excited LII is similar to that excited by a near-infrared beam $(1064 \mathrm{~nm})$.

LII measurement alone cannot provide the absolute value of SVF, since the proportionality constant $\left(C_{1}\right.$ in Eq. 20$)$ depends on many parameters requiring experimental calibration. Additional light extinction/spectral emission measurements [472], or absolute irradiance calibration [495, 496], are possible approaches that provide quantitative relations between LII intensity and absolute SVF.

When the LII signal is analyzed in a time-resolved manner, information about particle size can be obtained in addition to SVF. This is based on the fact that particles with different diameters are cooled down (after laser heating) at different rates [497]. Large particles have low surface area to volume ratio and thus are cooled relatively slowly compared to small particles. To obtain the quantitative relationship between LII signal decay rate and soot particle size, a model that describes the heat/mass 
exchange processes between the particle and the surrounding gases [498] is required. There are three major paths for energy loss: sublimation of soot particles, heat conduction to the surrounding gas and radiation of soot particles. Such energy losses can be corrected with the internal energy of soot particles through the following energy conservation equation [126]:

$$
\frac{d U}{d t}=\dot{\mathrm{Q}}_{a b s}+\dot{\mathrm{Q}}_{c o n d}+\dot{\mathrm{Q}}_{s u b}+\dot{\mathrm{Q}}_{r a d}
$$

where $U$ is the internal energy of soot particle, $\dot{Q}_{a b s}$ is the rate of laser energy absorption, $\dot{Q}_{c o n d}, \dot{Q}_{s u b}$, and $\dot{Q}_{\text {rad }}$ are the rates of energy loss due to heat conduction to surrounding gas, sublimation and heat radiation, respectively. The rate of change of soot internal energy can be written as:

$$
\frac{d U}{d t}=\frac{\pi}{6} d_{p}^{3} \rho_{s} c_{s} \frac{d T_{s}}{d t}=m_{p} c_{s} \frac{d T_{s}}{d t}
$$

where $\rho_{\mathrm{s}}, c_{\mathrm{s}}$, and $T_{\mathrm{s}}$ are the density, specific heat, and temperature of soot, respectively, and $m_{\mathrm{p}}$ is the mass of primary particle. The detailed size-dependent expression for $\dot{Q}_{a b s}, \dot{Q}_{c o n d}, \dot{Q}_{s u b}$ and $\dot{Q}_{\text {rad }}$ can be found in the review by Michelson [126], and they are generally dependent on primary particle sizes.

An important parameter in determining heat loss rates is soot temperature, which can be obtained by taking the ratio of soot radiation intensity at two different wavelengths (i.e., two-color pyrometry) [499]. With all the above information, a theoretical decay rate of soot radiation intensity can be computed, and comparison with an experimentally obtained rate would provide information on the primary particle size $\left(D_{\mathrm{pp}}\right)$ of soot under investigation.

In practice, time-resolved LII signals can be recorded by a photomultiplier tube (PMT) with low rise-time to provide high spatial resolution. Multiple PMTs can be used with different spectral filters installed to detect LII signals at different wavelengths, providing spectral information necessary for the retrieval of soot temperature [500]. Alternatively, planar LII can be performed using a high energy laser sheet as the excitation source and an ICCD camera as a signal detector. In this case, temporal resolution can be achieved by making measurements with consecutive gate delays in steady flames [501] and a single-shot measurement is also possible by using multiple ICCDs preset with different 
delay time [502]. The latter technique is essential for measurement in highly-transient turbulent flames [503].

\subsubsection{Thermophoretic sampling}

In addition to the optical techniques discussed above, thermophoretic sampling is another important method for obtaining information regarding size distributions, nanostructures, chemical compositions and various other important properties of collected soot particles. The thermophoretic effect refers to the drift of particles in a direction from high- to low- temperature regions. Thermophoretic sampling makes use of this particle drift to collect hot soot particles on cool probes. The collected particles can then be analyzed by various methods, including TEM and AFM. The rate of deposition can also be linked to local SVF so that accurate determination of the total mass of the sample can be an effective method for SVF measurement, as demonstrated by Köylü et al. [504]. This method is particularly attractive as it can determine SVF without prior knowledge of soot optical properties such as a refractive index.

One important consideration when performing thermophoretic sampling is the dwelling time of the probe inside a flame. If the dwelling time is too short, not enough particles can be collected for analysis. If the probe spends too much time in the flame, processes like thermal annealing may change the properties of the collected particles and the high temperature of flame may also break some more fragile probes, such as a TEM grid. In practice, rapid insertion of a probe through double-acting pneumatic, or electric-driven piston, is frequently used and the dwelling time can be accurately controlled to the range of tens of milliseconds $[485,505]$. In weakly sooting conditions, multiple insertions can be performed to collect enough soot samples [505].

It is noted that thermophoretic sampling is seldom performed in CDFs [506] compared to its wider application in coflow or premixed flames. This could be explained by the fact the very narrow sooting region $(\sim 1 \mathrm{~mm})$ in CDF makes spatially-resolved thermophoretic sampling using conventional TEM 
grid very difficult, especially considering the large vibration of a probe (to which the TEM grid is attached) after its rapid insertion (confirmed by high-speed images [507]). An innovative solution could be to insert a fine $\mathrm{SiC}$ wire into a flame, with a small angle between the flame axis and the wire (tilted wire), as pioneered by Figura and Gomez [508] in the $\mathrm{PhD}$ thesis of Figura. In this way, the wire could cover the entire axial extent of the flame, and particles in different axial positions of the flame will deposit on different parts of the wire. The disadvantage with this approach is that, due to the presence of the SiC wire (which blocks the electron beam in the TEM analysis), the sampled particles can only be viewed from the side. Recently, Carbone et al. [54] improved this SiC wire sampling technique and successfully applied it in a premixed flame, producing quantitative results (size distribution and volume fraction) via scanning electron microscopy (SEM) and helium ion microscopy (HIM) analysis of the soot samples.

Particle sampling is inherently more difficult for high-pressure flames due to the presence of a pressure chamber and associated limited physical access. The samples need also be transferred out of the chamber for further analysis after each collection, which may involve time-consuming depressurization and re-pressurization procedures. To address this issue, Vargas and Gülder [509] developed a multi-probe sampling system housed inside the pressure chamber including a circular sampling disk with ten extent-adjustable probe arms. A motor drive with programmable control unit determines which probe is used and the circular velocity of the sampling arm — and thus the sampling time. After each sampling process, the relevant probe can be retracted and the samples stored. When all the probes are loaded by samples, the experiment can be stopped, and the chamber is depressurized for sample retrieval.

A TEM analysis of sampled particles can provide information on the morphology of the soot aggregate and sizes along with the internal structures of primary particles [510]. In addition, if enough aggregates are sampled, statistical information on the particle size distributions can also be obtained. These are important information complementary to what can be obtained from optical techniques. 
Nevertheless, it is worth pointing out that through a combination of multiple diagnostic methods, Zhao et al. [505] demonstrated that nascent soot particles are liquid-like and are largely transparent to the electron beam of TEM. As a result, the TEM image contrast of these nascent particles is rather small. In addition, the evaporation of soot materials, as well as carbon crystallization and annealing, was also observed to be caused by the interaction of the soot sample and the high-energy electron beam of TEM $[511,512]$. These side effects make it a challenge to measure nascent soot particles with diameters below $10 \mathrm{~nm}$ with TEM [511]. Furthermore, as the nanostructure of nascent particles may be altered under the irradiation of high-energy electrons, results from HRTEM should be analyzed with caution.

HIM is a promising alternative to TEM in measuring nascent soot particles. With much less damage or alteration made to the sample, HIM can detect nascent soot particles with higher contrast [54, 511]. Furthermore, HIM was reported to identify particles as small as $2 \mathrm{~nm}$ [511], notably extending the lower size limit detectable by TEM (typically $10 \mathrm{~nm}$ ). Nevertheless, it should be noted that HIM cannot provide information of the inner structures of samples.

If larger amounts of soot particles are required, a water-cooled suction tube can be used, and the particles deposited on the cool tube walls, or filters placed inside the tube can then be extracted by solvents [125]. The soluble material can be studied by liquid chromatography, UV absorption and fluorescence [125] while the particles can be further analyzed by — for example — a thermogravimetric analyzer (TGA) for the oxidation characteristics, X-ray diffraction (XRD) for crystalline structures, and Raman spectroscopy and Fourier transform infrared spectroscopy (FTIR) for chemical properties.

\subsubsection{Differential mobility analysis}

Differential mobility analyzer (DMA) is becoming an increasingly popular tool for the determination of size distributions of flame-generated soot particles $[18,49,52,54,125,355,513$ 515]. DMA, as a size-selecting device, is usually adopted in combination with a particle counter, 
constituting a scanning mobility particle sizer (SMPS) system. In application, soot-laden flame gases were first sampled and diluted within a probe and then charged by a neutralizer with known charge distributions. In an external electric field, the charged particles would move to an electrode of opposite charge at different speeds that are dependent on their electric mobility and the electric field strength. By scanning the strength of the external electric field, particles with different mobility sizes can be separated in a similar manner as in a mass spectrometer, and subsequent measurement in a particle counter provides number concentrations of the size-selected particles. After correcting the mobility size for true particle size [516-518], a size distribution can be obtained.

Either an electrometer [54] or a laser optical counter (based on light scattering by particles) can be used to determine the number density of the classified particles. In the latter case, condensation growths of these tiny particles are required before they can be counted optically, due to the insensitivity of the laser-based method to nano-sized particles. Condensation particle counter (CPC) [519] is widely used for effectively counting nano-sized particles and a detailed description of its working principle can be found in Ref. [520]. A brief description is as follows. A particle-laden flow is brought into contact with a supersaturated vapor of a working fluid, which condenses on the particles so as to increase their sizes to an extent enough for optical detection. The level of supersaturation of the condensing vapor is of critical importance to the lower detection limit of particle sizes. With the increase in the supersaturation level, progressively smaller particles can be activated. On the other hand, however, if the supersaturation level is too high, homogeneous nucleation of the vapor itself will take place, interfering with the target particles and thus leading to false counts. Iida et al. [521] pointed out that the use of working fluids with high surface tension and low saturation vapor pressure is helpful to extend the lower limit of detectable particle size. Commercial instrument typically uses water, iso-propanol or n-butanol as the working fluid with detection limit down to $2.5 \mathrm{~nm}$ (e.g., TSI 3776 Ultrafine CPC with n-butanol). More recently, a diethyl glycol (DEG)-based condensation nano-enhancer, when used in combination with a second- 
stage growth booster, has been demonstrated to be capable of detecting particles with sizes down to $1 \mathrm{~nm}$ [522]. Through a theoretical investigation, de la Mora [523] anticipated that water at low temperature could even activate particles with diameter as small as $0.6 \mathrm{~nm}$.

An important consideration when using an SMPS to measure probe-sampled soot particles is to avoid particle losses (e.g., through particle coagulation or diffusion to the wall) in the sampling probe and transfer tubes. For example, it was shown that a loss of $10 \%$ of particles can occur in $20 \mathrm{~ms}$ for a typical flame gas that contains $10^{10}$ particles per $\mathrm{cm}^{3}$ [318]. As such, immediate dilution of the sampled gas is of paramount importance [49]. This means that a large amount of diluent must be mixed with the sampled flame gas immediately, posing significant challenges for the design of a sample system. A proven methodology was demonstrated by Wang and coworkers [48, 97, 230], who utilized a tubular probe, crossing a flame with a small pin hole drilled to sample the flame gas, while a carrier gas of fixed flow rate served as the diluent. Since a large flow rate of the diluent is required, the tubular probe must be relatively large (typically a tube with $1 / 4$-inch OD is used) so that it may significantly affect the flow field and the flame. To address this issue Abid et al. [48] proposed, for applications in burner-stabilized flat premixed flames, to conceal the probe in a stagnation plate, which also serves as a stabilizing plate and a downstream boundary. As long as this downstream boundary can be accounted for in accompanying numerical simulations, the presence of the probe posed no problems. Nevertheless, this proposed method is only applicable to premixed burnedstabilized flames. For other types of flames, significant challenges still exist in the design of suitable sampling systems with minimal influence on the flame itself. This challenge may explain why the majority of SMPS flame measurements were performed for premixed flames, while limited studies for coflow diffusion were mainly performed at the flame tip [515].

Particle size distribution, measured using SMPS, can be a rich source of information on various soot formation processes, as detailed in a previous section. Singh et al. [322] (also summarized in Ref. [18]) showed that various features of a size distribution function are directly related to-for 
example-nucleus size, nucleation, surface growth, and coagulation rates. In this regard, and also as pointed out by Wang [18], many soot model parameters can be fine-tuned and made more practical by validation against the experimentally measured size distributions.

There are additional techniques for soot characterization, such as dynamic light scattering, X-ray scattering, neutron scattering, aerosol mass spectrometry, etc. The scope of this review does not allow for further discussion of these techniques. More details can be found in excellent summaries by Kohse-Höinghaus and Jeffries [524], D’Anna [125], Wang [18], Desgroux et al. [127], and Michelsen [128].

\section{Sooting structures and soot evolution in counterflow flames}

Knowledge about sooting structures is necessary for a fundamental understanding of soot evolution in flames. As such, we discuss in this section the general flame and sooting structures in CDFs, along with their differences/similarities to those in coflow diffusion flames. We then highlight the response of soot evolution to flame structure variations, which is followed by a discussion on the effects of strain rate on sooting processes in CDFs. This section concludes with a description of soot formation in partially-premixed counterflow flames.

\subsection{Sooting structures of CDFs and comparison with coflow diffusion flames}

In a counterflow flame, the nozzle exit velocities for the fuel and oxidizer streams are with opposite signs. Therefore, somewhere between the nozzles there always exists a location with zero axial velocity, that is the stagnation plane. The relative positions between the high-temperature reaction sheet and the stagnation plane have a strong influence on soot evolution in CDFs. Specifically, sooting CDFs can be differentiated into two distinctive types based on this relative position [73]: soot formation flames and soot formation/oxidation (SFO) flames. The schematics shown in Figure 9 help to clarify this point. 

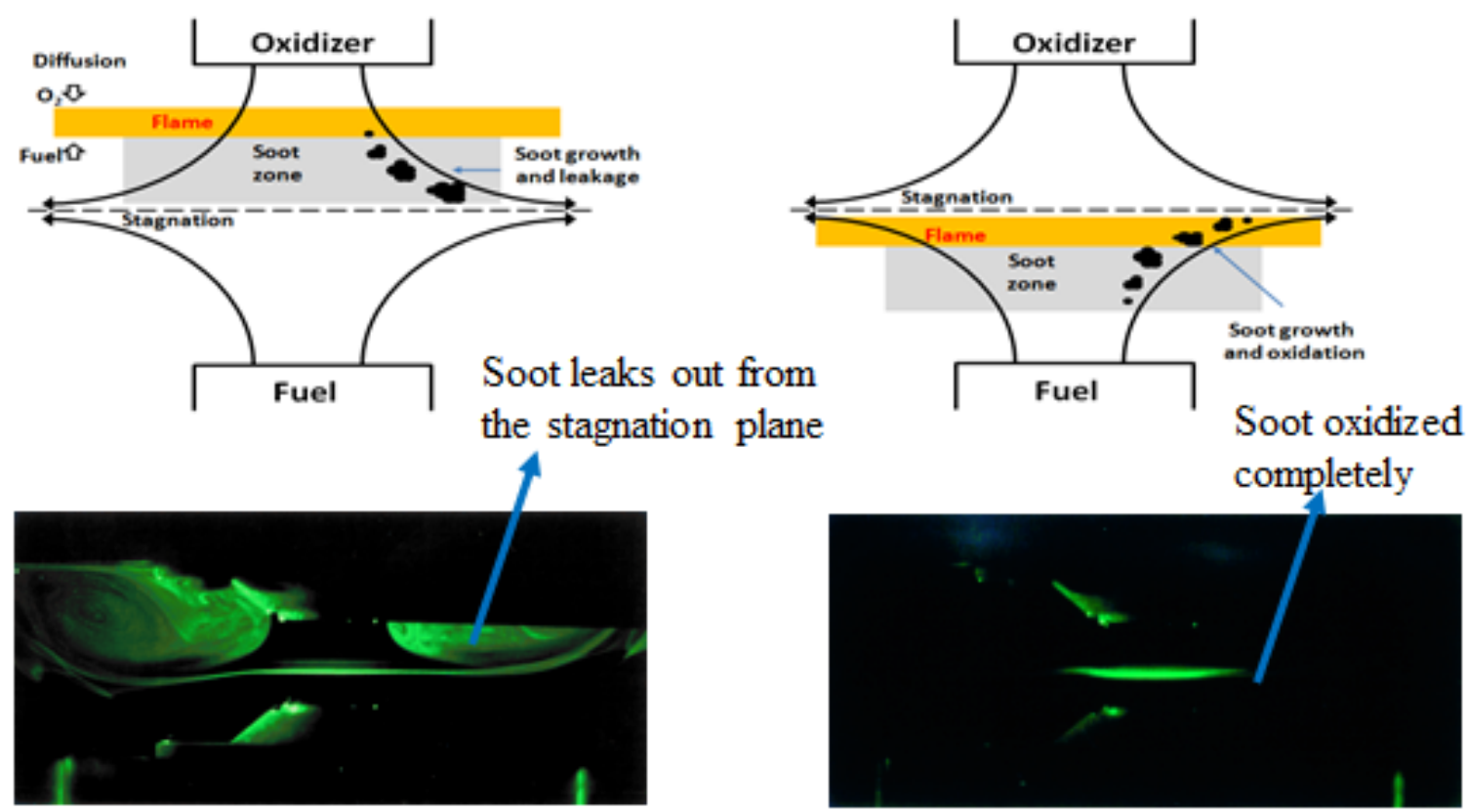

\section{a) Soot formation (SF) flame \\ b) Soot formation/oxidation (SFO) flame}

Figure 9. Schematics of evolution for soot formation (SF) flame located on the oxidizer side of the stagnation plane (a) and soot formation/oxidation (SFO) flame on the fuel side of the stagnation plane (b). Mie scattering images are also included to show soot leakage (thus emission) through the particle stagnation plane in SF flame. In SFO flame, initially formed soot can be oxidized completely, so no soot emission can be observed [73].

Figure 9a depicts a situation when the flame is located on the oxidizer side of the stagnation plane. In such a case, soot particles, which are incepted on the fuel side from the flame sheet, will be convected away from the flame towards the stagnation plane, without passing through the oxidative high temperature flame region. While being transported, these soot particles will continue to grow through surface reactions and particle-particle coagulations. They will eventually leak out radially to the environment, because it is unlikely for these large particles to diffuse across the stagnation plane. Soot oxidation is not present during this process, since oxidizing species such as $\mathrm{OH}$ and $\mathrm{O}_{2}$ only exist with considerable concentrations on the oxidizer side of, or near, the flame sheet. As a result, such flames are termed soot formation flames. In contrast, when the flame sheet is located on the fuel 
side of the stagnation plane (Figure 9b), soot particles are convected towards the flame, where intensive soot oxidation by $\mathrm{O}_{2}$ and $\mathrm{OH}$ radicals occur. In many cases, the initially formed soot particles can be completely oxidized, so no soot emission can be observed out of the flame. This type of CDF is termed the soot formation/oxidation flame.

More detailed flame and sooting structures for SF/SFO flames were investigated by Kang et al. [73]. The axial profiles of temperature, fuel, oxygen, and important soot precursor concentrations, as well as those of SVF, average soot particle size and number density, are shown in Figure 10 for typical SF and SFO flames fueled by propane.

For the SFO flame $\left(X_{\mathrm{F}, 0}=0.25\right.$ and $X_{\mathrm{O}, 0}=0.7$, where $X$ is the mole fraction, the subscripts $\mathrm{F}$ and $\mathrm{O}$ indicate fuel and oxygen in the fuel and oxidizer streams, respectively; the subscript 0 is the freestream, and $z$ is the distance from fuel nozzle) shown in Figure 10(a-d), the location of the flame sheet (represented by the maximum temperature location $z_{\mathrm{T}, \max }$ ) stays close to the stagnation plane $\left(z_{\text {stg,p }}\right)$. Here, the difference between the particle and gas-phase $\left(z_{\text {stg }}\right)$ stagnation planes can be attributed to the thermophoretic effect. Fuel pyrolysis and PAH growth occur on the fuel side of the particle stagnation plane (also of the flame sheet), confirmed by the profiles of $\mathrm{C}_{2} \mathrm{H}_{2}$ mole fraction (a) and the PAH fluorescence signal $Q_{\mathrm{f}}(\mathrm{b})$. The sooting zone, indicated by the light scattering signal $Q_{\mathrm{vv}}$, is also located in this region (b). It is noted that $Q_{\mathrm{vv}}$ begins to rise near $z=5 \mathrm{~mm}(\sim 1700 \mathrm{~K})$, indicating the initiation of soot inception at this position. The coincidence of the location of peak PAH fluorescence (represented by $Q_{\mathrm{f}}$ ) with the location at which soot inception begins may indicate the critical role of PAHs for soot inception. As the formed particles are convected (to the right) towards the particle stagnation plane, their number density continues to decrease (c), due primarily to particle-particle coagulation. Meanwhile, the average particle size $D_{63}$ (c) and soot volume fractions increase through surface growth and coagulation (d), reaching their peaks near $z=6 \mathrm{~mm}$, and then decreasing due to oxidation. Note an appreciable amount of $\mathrm{OH}$ radicals exists on the fuel side of the flame sheet (a), where the soot zone is situated. 


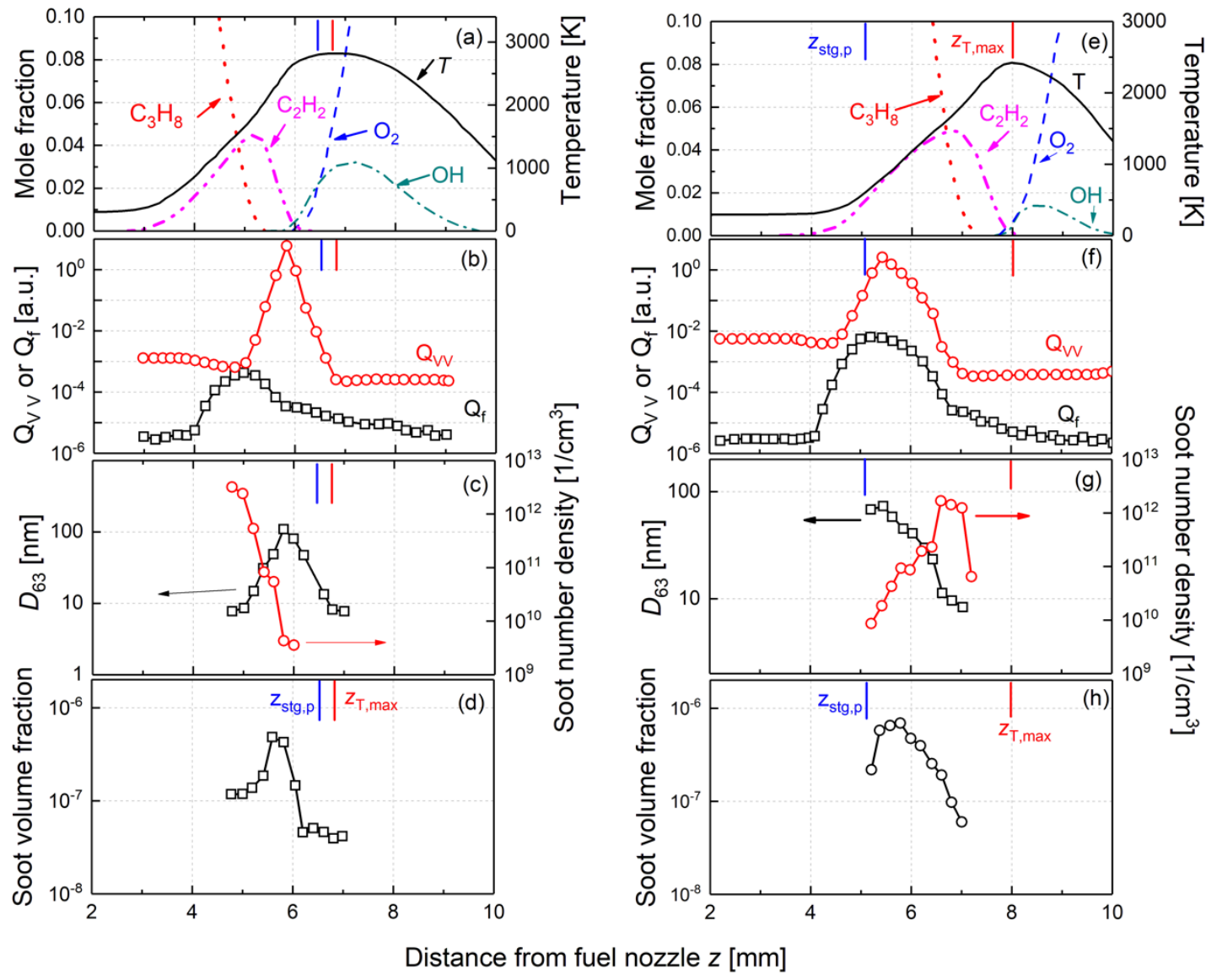

Figure 10. Flame and sooting structures of SFO (a,b,c,d) and SF (e,f,g,h) flames, adapted from Kang et al. [73] with permission of Elsevier.

In the SF flame $\left(X_{\mathrm{F}, 0}=1.0, X_{\mathrm{O}, 0}=0.25\right)$ shown in Figure $10(\mathrm{e}-\mathrm{h})$, the flame front is spatially separated from, and on the oxidizer side of, the stagnation plane. $\mathrm{C}_{2} \mathrm{H}_{2}$ and $\mathrm{PAH}$ fluorescence are distributed between the stagnation plane and the flame sheet $(e, f)$. The peak of the number density profile and the onset of $Q_{\mathrm{vv}}$ are located near $z=7 \mathrm{~mm}$, where soot inception occurs. These newlyincepted soot particles are then convected towards the stagnation plane (away from the flame sheet). Following the direction of this convection transport (to the left), soot number density (g) decreases, but average particle size (g) and soot volume fraction (h) increase, through both coagulation and surface growth, reaching their peaks at the stagnation plane. In this process, soot oxidation hardly occurs because few $\mathrm{OH}$ radicals exist in the sooting zone of $z=5-7 \mathrm{~mm}(\mathrm{e})$. Note, it was observed $[242,281,407,525,526]$ that a SF flame visually exhibited three distinct zones from the fuel side to 
the oxidizer: an orange zone, a luminous yellow zone on the fuel side of the flame, and a blue flame reaction zone. This is consistent with the flame and soot zone structures shown in Figure 10 (e-f).

The above work of Kang et al. [73] offers general knowledge on sooting characteristics of SF and SFO flames, which were further investigated through measurements of soot precursor (PAH) concentration, soot volume fractions [281, 372, 447, 527, 528], and through numerical simulations using detailed kinetic mechanisms [87, 380]. From these investigations, major differences in the sooting structures between SF and SFO flames can be identified, which are summarized as follows.

In a SFO flame, considerable amounts of $\mathrm{OH}$ radicals exist on the fuel side of the flame sheet, overlapping with the sooting zone. The spatial proximity of soot and $\mathrm{OH}$ species means that significant soot oxidation can take place as soot particles (formed on the fuel side of flame) are transported by the convective flow towards the high temperature flame sheet. In a SF flame, on the other hand, there is a large spatial separation between the flame sheet and the stagnation plane, and the convection is in a direction away from the flame. Consequently, unlike in SFO flames, oxidizing species like $\mathrm{OH}$ and $\mathrm{O}_{2}$ do not coincide with the soot zone, so soot oxidation is absent [73]. These differences in soot evolution result in the distinct shape of SVF profiles between SFO and SF flames, as can be more clearly seen in Figure 11 [378]. In SFO flames, the profile of SVF is somewhat symmetric, indicating that the soot particles continuously experience inception, growth, and finally, destructive oxidation. The SVF drops to a negligible level before reaching the stagnation plane, consistent with the fact that SFO flames typically do not emit soot. In contrast, the SVF profile in SF flame is highly skewed toward the fuel side, characterized by a sharp decrease across the stagnation plane, a phenomenon observed also in several other studies $[78,281,529,530]$. This is because the particles have very low diffusivity and cannot effectively penetrate through the stagnation plane, so they eventually leak out of the flame in the radial direction.

Of special note is the observation by D'Anna and coworkers $[372,531]$ that in a $\mathrm{C}_{2} \mathrm{H}_{4} \mathrm{SF}$ flame $\left(X_{\mathrm{F}, 0}=0.75, X_{\mathrm{O}, 0}=0.22\right.$ diluted by $\left.\mathrm{Ar}\right)$, abundant small particles also exist on the fuel side of the 
stagnation plane. The presence of soot in this pyrolytic region can be explained, according to the authors, by the formation of soot nuclei through physical coagulation of PAHs under low temperature conditions. At first glance, this observation seems to be incompatible from the measurements of Xu et al. [378], in which negligible amount of soot exists on the fuel side of particle stagnation plane (Figure 11). However, it is important to realize that D'Anna's observation is consistent with the high soot number density on the fuel side of SF flames, as predicted by $\mathrm{Xu}$ et al. [378] through a detailed PAH-based soot model. The particles formed on the fuel side are not likely to experience significant mass-adding surface reactions due to the relatively low temperature in the region. Therefore, these particles are nascent and may have notably different optical properties compared to mature graphitic soot. They can even be liquid-like $[18,485]$ and transparent to visible/near infrared lights and so cannot be effectively detected by the visible or infrared light extinction method, as employed in the work of Xu et al. [378] and many others. On the other hand, these nanoparticles may well respond to the laser-induced emission spectrum method with the use of a UV laser (266 nm) as the excitation source, a technique frequently used by D’Anna and coworkers [372, 531].

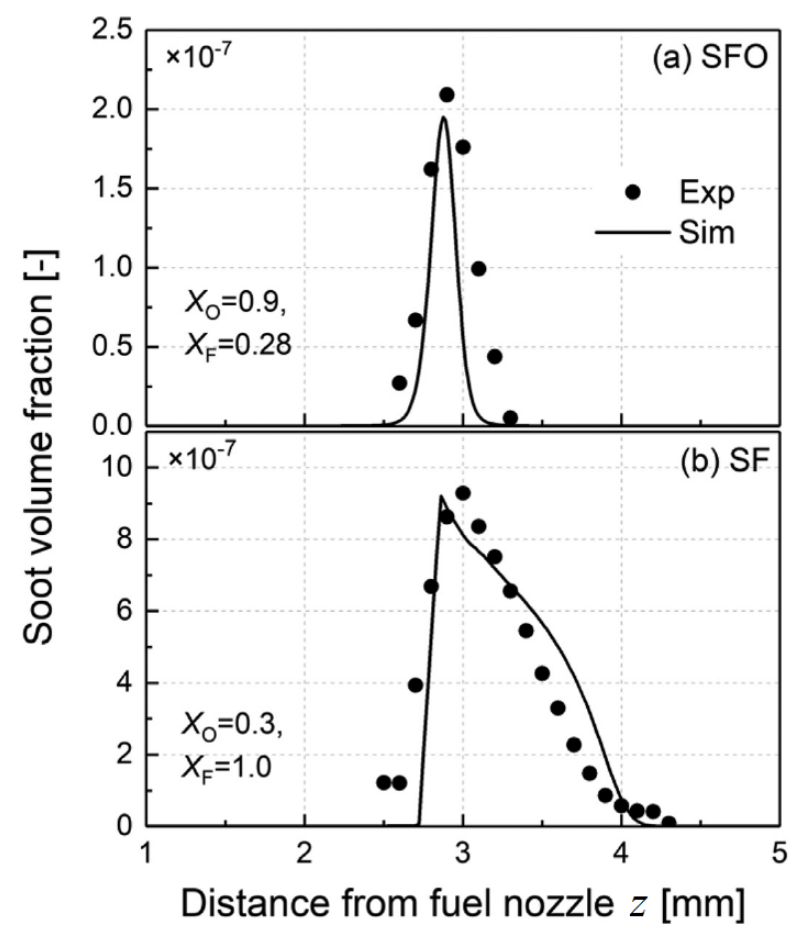


Figure 11: Experimental and computed soot volume fraction profiles of $\mathrm{C}_{2} \mathrm{H}_{4}$ for (a) SFO flame $\left(X_{\mathrm{F}, 0}\right.$ $\left.=0.28, X_{\mathrm{O}, 0}=0.9\right)$ and $(\mathrm{b}) \mathrm{SF}$ flame $\left(X_{\mathrm{F}, 0}=1.0, X_{\mathrm{O}, 0}=0.3\right)$, reprinted from Xu et al. [378] with permission of Elsevier.

Another interesting difference between SF and SFO flames regards the profiles of PAH LIF signals, which were observed to have double peaks in SFO flames, but only one peak in SF flames [281, 447, 528]. Figure 12 shows a comparison of the measured SVF and PAH fluorescence profiles between typical $\mathrm{C}_{2} \mathrm{H}_{4} \mathrm{SFO}$ (a) and SF (b) counterflow flames. In the SFO flames (a), a second increase of PAH fluorescence, starting at around $z=5 \mathrm{~mm}$, can be clearly seen. Considering that it occurs in the hightemperature soot oxidation zone, where soot oxidation by $\mathrm{OH}$ radicals leads to a decrease in SVF, Hwang et al. [281] tentatively attributed this second peak of LIF signal to PAHs generated by soot oxidative fragmentation.

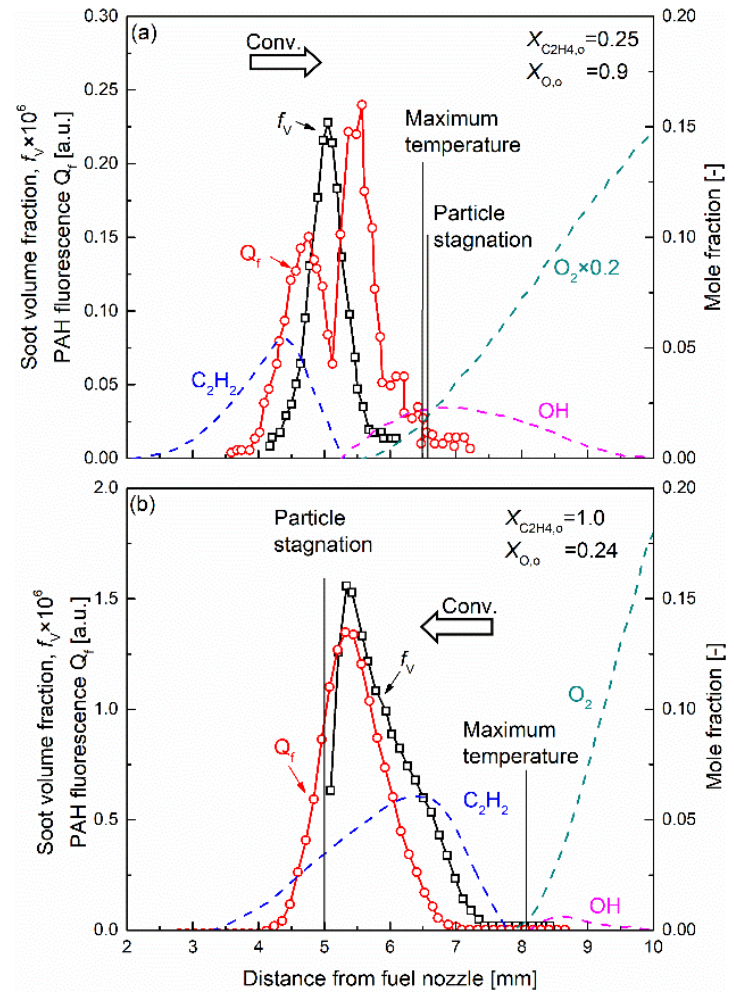

Figure 12: Profiles of soot volume fraction, PAH fluorescence in SFO (a) and SF (b) ethylene CDFs. Also shown are computed $\mathrm{C}_{2} \mathrm{H}_{2}, \mathrm{O}_{2}$ and $\mathrm{OH}$ mole fractions, reprinted from Hwang et al. [528] with permission of Elsevier. 
Kang et al. [73] further investigated the sooting structure of normal $\left(X_{\mathrm{F}, 0}=1.0, X_{\mathrm{O}, 0}=0.21\right)$ and inverse coflow diffusion flames $\left(X_{\mathrm{F}, 0}=0.3, X_{\mathrm{O}, 0}=0.6\right)$ and observed a similarity in sooting characteristics between normal (inverse) coflow diffusion flame and SFO (SF) flame. This similarity is clearly demonstrated in Figure 13, where direct photos and sooting structures of these flames are presented. For the normal coflow flame (Figure 13a, central fuel jet surrounded by coflowing oxidizer), PAHs are formed in the fuel-rich pyrolysis zone near the fuel nozzle, and are then incepted to form soot particles, which are spatially distributed downstream of the PAH region. Soot particles are then transported further downstream towards the high temperature reaction zone, where highly oxidative $\mathrm{OH}$ radicals are abundant. Consequently, the axial profile of SVF and particle size both exhibited a rise-then-fall behavior with the increase in axial distance above the nozzle [62, 72, 323, 403, 532-534], similar to the case for a CDF of the SFO type.

For an inverse coflow flame (Figure 13b, central jet of oxidizer surrounded by coflowing fuel), the LIF and LII images obtained by Mikofski et al. [535] showed that the formation of PAHs and the soot zone occurred close to the flame wing, and the sooting zone almost completely enclosed the central reaction sheet-a fact that can also be visually confirmed by the relative position of the yellow luminous region and the blue flame zone in Figure 13d. As a result, after formation, soot particles are convected away from the high temperature flame sheet and are eventually emitted out from the flame. This evolution process is similar to that in SF CDFs; it also explains the experimental fact that the radially integrated SVF of inverse coflow increases and approaches a constant value downstream of the flame (i.e., no oxidation) $[535,536]$. These studies demonstrated the important role of flow convection in the evolution of soot particles and thus in affecting the overall sooting structures of diffusion flames. 


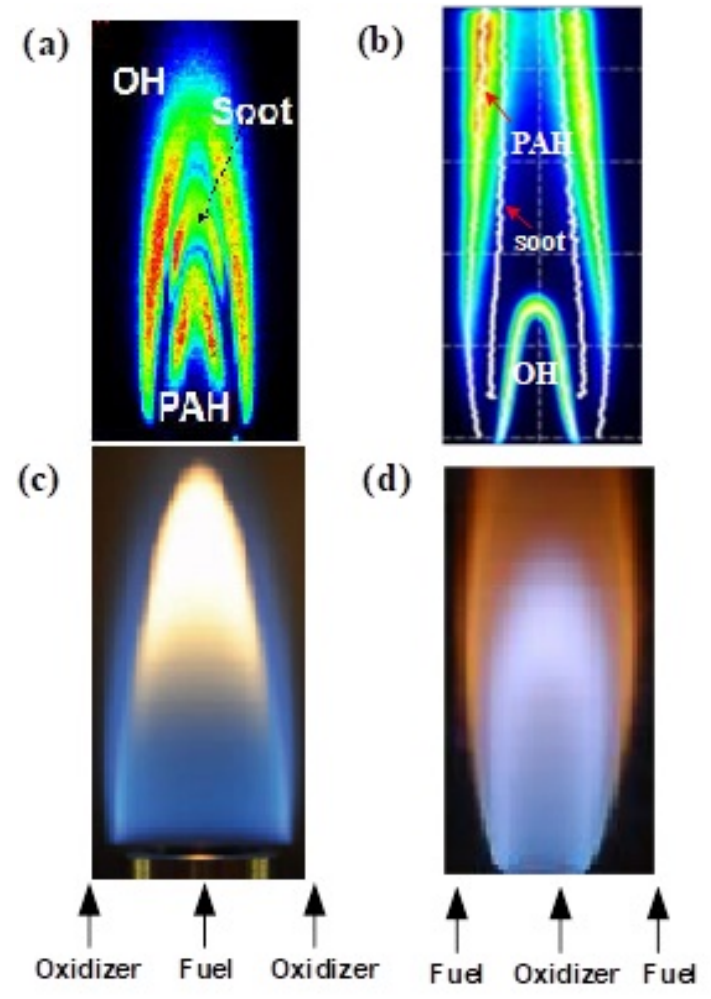

Figure 13. Sooting structures as visualized by OH LIF, PAH LIF and soot LII for normal (a) and inverse (b) coflow diffusion flames, reprinted from Mikofski et al. [535] with permission of Elsevier. Also shown are direct photos of normal coflow flame (c), reprinted from Bisetti et al. [537] with permission of Elsevier and inverse coflow flame (d), reprinted from Wang et al. [538] with permission of Elsevier.

\subsection{Influences of stoichiometric mixture fraction on sooting structures in CDFs}

The above discussions on the differences in soot evolution between SF and SFO flames highlight the important role of flame location in determining the sooting behavior in CDFs. The variation of flame location can be achieved by adjusting the dilution level in the fuel and/or oxidizer streams. The extent can be quantified by the stoichiometric mixture fraction, $Z_{\mathrm{st}}$, defined as $Z_{\mathrm{st}}=(1+$ $\left.Y_{\mathrm{F}, 0} W_{\mathrm{O}} v_{\mathrm{O}} / Y_{\mathrm{O}, 0} W_{\mathrm{F}} v_{\mathrm{F}}\right)^{-1}$. Here, $Y$ represents the mass fraction, $W$ is the molecular weight, $v_{\mathrm{F}}$ and $v_{\mathrm{O}}$ are fuel and oxygen stoichiometric coefficients, respectively. Therefore, the value of $Z_{\text {st }}$ will change when fuel or oxidizer compositions vary, indicating the change of flame structure. A typical SF flame with 
undiluted fuel/air combustion (SFO flame with diluted fuel burning in pure oxygen) corresponds to a low (high) $Z_{\text {st }}$ value.

Atreya et al. [539] investigated the effect of $Z_{\text {st }}$ on soot formation in a series of low strain rate methane CDFs (effects of strain rate to be discussed in Section 4.3). The sooting structures of four flames, with $Z_{\text {st }}$ increasing from 0.129 to 0.584 , are compared in Figure 14 . The data showed that as $Z_{\mathrm{st}}$ increased, the flame front $\left(z_{\mathrm{T}, \max }\right)$ moved progressively towards the fuel side, along with the shift of the stagnation plane $\left(z_{\mathrm{stg}, \mathrm{p}}\right)$ from the oxidizer to the fuel side. Such change of flame location notably affected the structures of the soot zone in many aspects. First, the flame with $Z_{\mathrm{st}}=0.129$ (a) had the largest soot zone thickness but the smallest average temperature in the soot zone; Second, SVF increased monotonically towards the stagnation plane (following the direction of particle convection) in flames with $Z_{\mathrm{st}}=0.129$ and $0.297(\mathrm{a}, \mathrm{b})$-cases in which the reaction sheets were located on the oxidizer side of $z_{\text {stg,p. }}$. However, the SVF profiles exhibited a rise-then-fall behavior for flames with $Z_{\mathrm{st}}=0.48$ (c) and $0.584(\mathrm{~d})$, where the reaction front was either close to, or on the fuel side of, $z_{\text {stg,p. }}$. These results were consistent with the aforementioned sooting characteristics of typical SF and SFO flames; Third, particle number density $(N)$ decreased continuously toward the stagnation plane for $Z_{\text {st }}$ $=0.129$, although it varied non-monotonically with $z$ for the remaining three flames with larger $Z_{\text {st }}$. This result suggests that the soot inception process can be strongly affected by the spatial shift of $\mathrm{OH}$ radicals towards the sooting zone with increasing $Z_{\text {st. }}$ As Atreya et al. [539] explained, this behavior was also compatible with the fact that the calculated $\mathrm{OH}$ mole fraction (without soot model) agreed well with the measured one for $Z_{\mathrm{st}}=0.129$, but obviously higher than the measured for cases with $Z_{\mathrm{st}}$ $=0.297$ and $0.48-$ suggesting a fraction of $\mathrm{OH}$ radicals may be consumed for the oxidation of soot particles in the latter cases. 

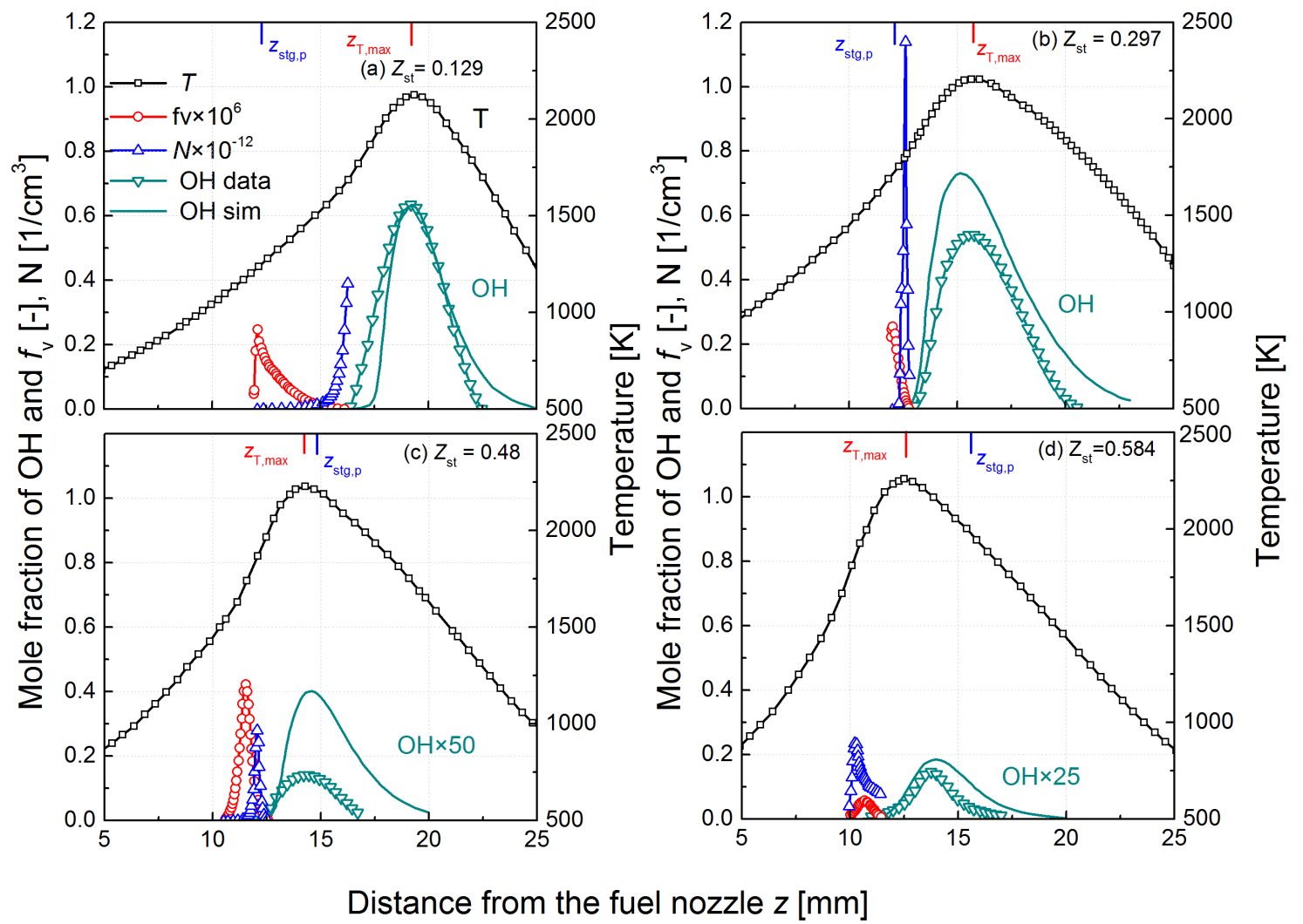

Figure 14. Flame and sooting zone structures for methane CDFs with varying $Z_{\text {st. }}$ Data taken from Atreya et al. [539].

Also note in Figure 14, peak SVFs showed a non-monotonic variation with $Z_{\text {st }}$, with the flame of $Z_{\mathrm{st}}=0.48$ having the highest peak SVF. This phenomenon is different from the observations in Refs. [377, 540-545], where peak SVF decreased monotonically with the increase in $Z_{\text {st. }}$ Such differences may derive from the fact that the adiabatic flame temperatures remained unchanged as $Z_{s t}$ varied in Refs. [377, 540-545], but they were not controlled by Atreya et al. [539]. With fixed peak temperature, results from Refs. [377, 540-545] generally showed that the inhibition of soot and their precursors with increasing $Z_{\text {st }}$ can be attributed to both the hydrodynamic effects and the alteration of flame structure.

The hydrodynamic effects refer to the directional change of soot convection when $Z_{\text {st }}$ changes [542]. The change in flame structure with $Z_{\text {st }}$ (i.e., the relationship between the temperature and local species concentration) may also affect soot formation significantly, as demonstrated by Du and Axelbaum 
[540] through detailed experimental and numerical studies of ethylene CDFs. In their study, the sooting tendency of the CDFs decreased monotonically as $Z_{\text {st }}$ increased, attributed by the authors to the result of flame structure variation. Note, since only critical sooting condition was considered, the flames studied were only relevant to soot inception (i.e., without particle growth/transport processes), and therefore were expected not to be sensitive to the hydrodynamic effects. The general flame structures for flames with $Z_{\text {st }}$ of 0.064 and 0.78 are compared in Figure 15. As seen, the flame with higher $Z_{\mathrm{st}}$ had lower fuel (marked as $\left.Y_{\mathrm{C} 2 \mathrm{H} 4}\right)$ but higher oxygen concentrations $\left(Y_{\mathrm{O} 2}\right)$ near the reaction zone. Du and Axelbaum argued that in flames with high $Z_{\text {st }}$, the low fuel concentration tended to slow down fuel pyrolysis and soot precursor formation. In addition, the shift of oxygen concentration towards the fuel side enhanced the oxidation of soot precursors, also contributing to a reduction of soot formation. Similar arguments have been made by other researchers [377, 546, 547]. Furthermore, the sooting zone was known to be constrained in a region bounded by the oxidizing flame sheet and the fuel-rich zone, with temperatures high enough for soot inception $[539,540]$. As $Z_{\text {st }}$ increased, the spatial shift of the oxidizing species towards the fuel nozzle reduced the thickness of the sooting zone until it reached a soot-free (permanently blue) condition [540].

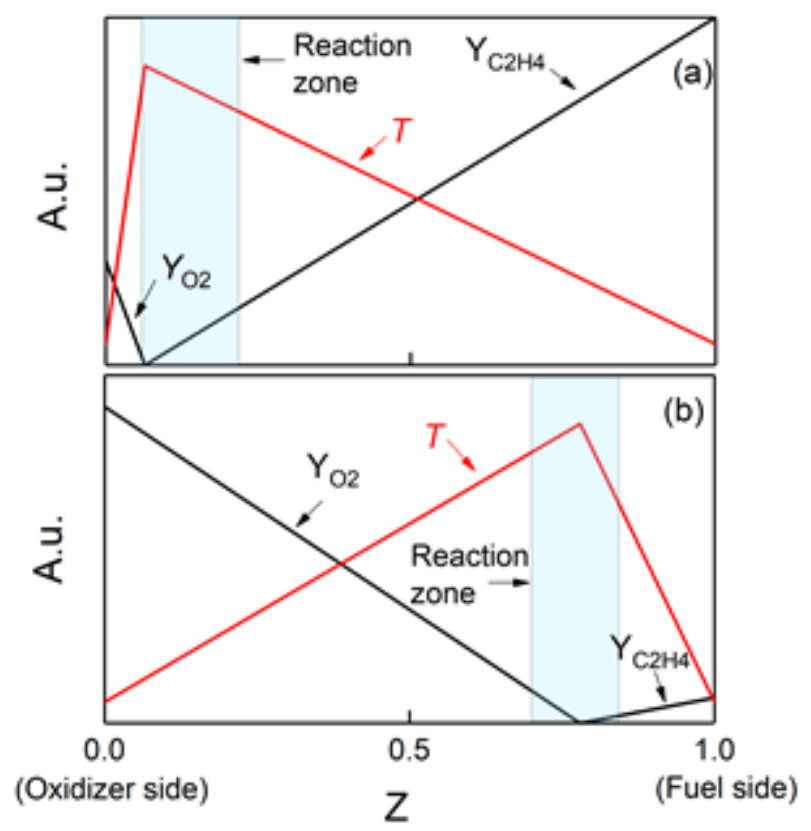


Figure 15. Schematic diagram of flame structures for (a) ethylene/air CDFs with $Z_{\mathrm{st}}$ of 0.064 and (b) diluted ethylene/oxygen CDF with $Z_{\text {st }}$ of 0.78 , redrawn from Du et al. [540] with permission of Elsevier.

Through numerical simulations with detailed chemistry accounting for aromatic species formation, Skeen et al. [543] attributed the soot-free phenomena in high $Z_{\text {st }}$ flame to the inhibited formation of soot precursors (i.e., benzene and phenyl). In their series of ethylene CDFs investigated, an increase in $Z_{\mathrm{st}}$ was seen to decrease the temperature in the fuel pyrolysis zone where abundant $\mathrm{H}$ radical existed, although the peak temperature remained constant. This, in turn, drove the dominant propargyl formation reactions $(\mathrm{R} 50)-(\mathrm{R} 51)$ in the reverse direction because both (forward) reactions were endothermic and produced $\mathrm{H}$ radical. Consequently, the formation of benzene and phenyl via propargyl self-combination was inhibited.

$$
\begin{array}{ll}
\mathrm{C}_{2} \mathrm{H}_{2}+\mathrm{CH}_{3} \Leftrightarrow \mathrm{pC}_{3} \mathrm{H}_{4} \text { (propyne) }+\mathrm{H} & \mathrm{R} 50 \\
\mathrm{pC}_{3} \mathrm{H}_{4} \Leftrightarrow \mathrm{C}_{3} \mathrm{H}_{3}+\mathrm{H} & \mathrm{R} 51
\end{array}
$$

The change of $Z_{\mathrm{st}}$ can result in flame structure variations in both physical distance and mixture fraction $(Z)$ space, which may make it somewhat difficult to interpret the effects of $Z_{\text {st }}$ on sooting behavior. In such a context, Axelbaum and coworkers $[77,548]$ proposed to understand the flame structure effect in $\mathrm{C} / \mathrm{O}$ ratio space. The parameter of $\mathrm{C} / \mathrm{O}$ ratio can also indicate the local mixing process, and unlike the mixture fraction $Z$, it is insensitive to variation in fuel/oxidizer boundary composition. As shown in Figure 16a, when plotting the flame structure in terms of $\mathrm{C} / \mathrm{O}$ ratio, the flame sheet is always located at $(\mathrm{C} / \mathrm{O})_{\mathrm{st}}$, which has a numerical value of $1 / 3$ for ethylene fuel, regardless of $Z_{\mathrm{st}}$. In contrast, the flame location changed notably with $Z_{\mathrm{st}}$ when expressed in $Z$ space (see Figure 15). As an example of application, Xia et al. [77] analyzed the soot-free phenomena in high $\mathrm{Z}_{\mathrm{st}}$ flame reported by Skeen et al. [543]. Unlike the case where a considerable shift of H radical and temperature profiles occurred in the physical distance space [543], the edge of the H profile (falloff) remained at nearly the same $\mathrm{C} / \mathrm{O}$ ratio of 0.5 (Figure 16b). Therefore, it was straightforward to observe the reduction of flame temperature at $\mathrm{C} / \mathrm{O}=0.5$ when $Z_{\mathrm{st}}$ increased. In this regard, the 
conclusion that it is the decrease of flame temperature in the $\mathrm{H}$ radical abundant region that causes the reduction of soot precursors in the high $Z_{\text {st }}$ flame, becomes more evident.

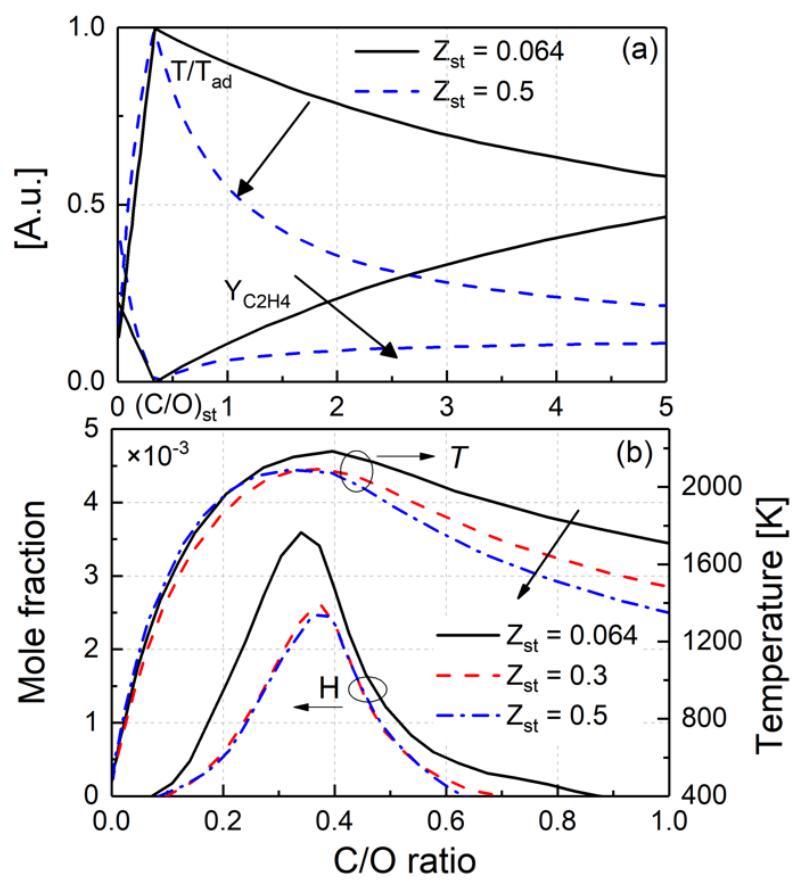

Figure 16: Global flame structure (a) and temperature and $\mathrm{H}$ radical profiles (b) of ethylene flames in $\mathrm{C} / \mathrm{O}$ ratio space with different $\mathrm{Z}_{\text {st. }}$. Adapted from Xia et al [77] with permission of Elsevier.

\subsection{Influences of strain rate on sooting structures in CDFs}

Due to an accompanying significant entropy decrease [18], soot formations is a highly reversible and rate-limiting process; therefore it can be significantly affected by flow residence time. The characteristic flow time for flames under aerodynamic straining (i.e., CDF) is strain rate, which is essentially the gradient of axial velocity and has a unit of inverse time [549]. Considering that strain rate is a defining parameter for $\mathrm{CDF}$, it is important to understand how it can affect sooting structures in CDFs.

Before discussing its effects, it is worthwhile to first clarify the definition of strain rate, since different researchers quantify this parameter in different ways. As a matter of fact, there is no true unique definition of strain rate in the counterflow, partly because of the axial variation of velocity 
gradient [550]. For studies with direct measurement of the axial flow field, either with LDV or PIV, it is a common practice to define the characteristic strain rate as the absolute value of the measured axial velocity gradient in the thermal mixing layer of the oxidizer stream $[78,462]$ (the oxidizer stream is chosen because most CDFs feature a very low stoichiometric mixture fraction so that the flame front is typically located on the oxidizer side of the stagnation plane). Figure 17 shows a typical axial velocity profile as measured by Du et al. [551]. The strain rate was found by fitting the experimental data with a straight line, as shown by the dashed line in the figure. This experimentallydetermined strain rate will hereafter be referred to as the local strain rate $\left(K_{\mathrm{L}}\right)$. In the absence of experimental data, the strain rate may be estimated from nozzle exit velocities $\left(V_{\mathrm{i}}\right)$ and nozzle separation distances $(L)$, where $i$ can be either fuel $(\mathrm{F})$ or oxidizer $(\mathrm{O})$ stream. The simplest approach is to assume a potential boundary condition and write the strain rate as $\left(V_{\mathrm{F}}+V_{\mathrm{O}}\right) / L$. This definition will hereafter be referred to as the global strain rate $\left(K_{\mathrm{G}}\right)$. It should be pointed out that the velocity boundaries of CDFs that employ nozzles with porous plugs are more like the plug flow type, and a more relevant description of the strain rate $\left(K_{\mathrm{E}}\right.$, effective strain rate) was provided by Seshadri and Williams [550, 552] as:

$$
K_{E}=\frac{2 V_{O}}{L}\left(1+\frac{V_{F}}{V_{O}} \sqrt{\frac{\rho_{F}}{\rho_{O}}}\right)
$$

In the derivation of Eq. 23, in addition to employing a plug flow velocity boundary, it was further assumed that the viscous mixing layer could be treated as a thin sheet at the stagnation plane (i.e., large Reynolds number) [553]. Although experimentally determined $K_{\mathrm{L}}$ is preferred, $K_{\mathrm{G}}$ and $K_{\mathrm{E}}$ also provide a convenient way to select corresponding experimental conditions for parametric studies on strain rate effects, as long as the definition is consistent. To avoid confusion when results from different studies are compared with different strain rate definitions, an explicit definition of which strain rate was employed will be offered, using the corresponding symbols. In the present section, only the effects of strain rate on overall sooting structure will be discussed; detailed parametric studies 
will be reviewed in Section 5.5, where the unsteady response of soot formation to time-varying strain rates are also described.

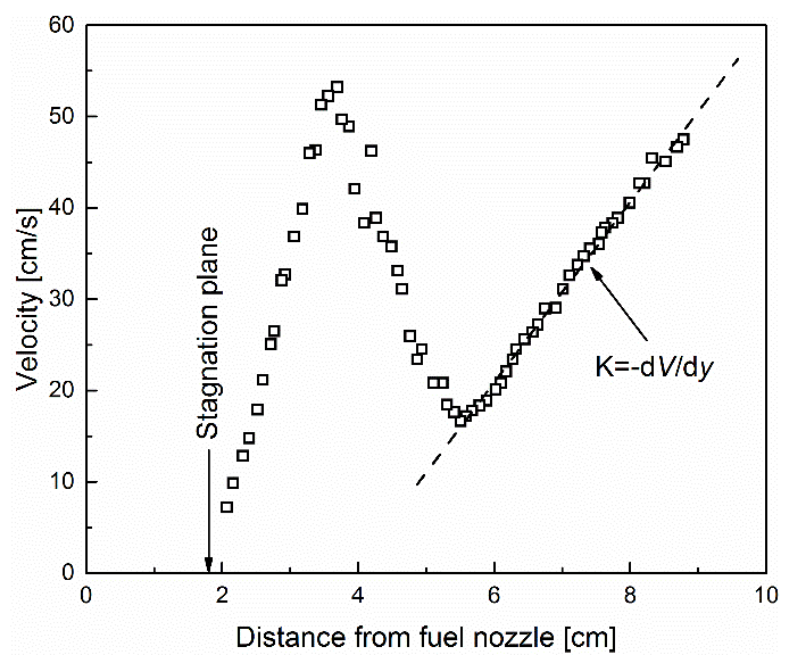

Figure 17. Measured axial velocity profiles in counterflow diffusion flames and determination of local strain rate. Reprinted from Du et al. [551] with permission of Elsevier.

The strong effect of strain rate on sooting structures can be visualized in Figure 18, where the axial profiles of SVF, average particle size, and soot growth rate for a series of $\mathrm{C}_{2} \mathrm{H}_{4} \mathrm{CDFs}\left(X_{\mathrm{F}, 0}=1.0\right.$, $X_{\mathrm{O}, 0}=0.25$ ) are shown [91]. With the nozzle exit velocity $V_{0}$ increasing from $15 \mathrm{~cm} / \mathrm{s}$ to $30 \mathrm{~cm} / \mathrm{s}, K_{\mathrm{E}}$ varied from 75 to $150 \mathrm{~s}^{-1}$.

As can be seen, in addition to the decrease in peak SVF (a), the soot zone thickness decreased monotonically, consistent with the shrinkage in flame thickness as strain rate increased. Indeed, accompanying numerical simulation showed that with the increase in strain rate, the flame sheet and the stagnation plane were brought closer together, leaving an increasingly narrower region for soot growth. Average soot diameters also decreased (b), partially explained because the increased strain rate reduced time available for coagulation and surface growth between the soot inception zone and the particle stagnation plane. The volumetric soot mass growth rate $\left(\omega_{\mathrm{v}}\right)$ also decreased with the increase in strain rate, caused primarily by the reduced surface area in the more strained flames. However, the soot mass growth rate per unit surface area $\left(\omega_{\mathrm{A}}\right)$ showed an interesting behavior. In 
regions close to the flame front $\omega_{\mathrm{A}}$ was higher for flames with higher strain rate, while the trend became reversed in regions adjacent to the stagnation plane. The reason lies in the effects of strain rate on temperature profiles. Although the peak temperature was reduced due to flame weakening as the strain rate increased, in regions closer to the stagnation plane, where soot surface growth peaks, the flame with higher strain rate actually had a higher temperature. Nevertheless, the effects of this higher $\omega_{\mathrm{A}}$ were overwhelmed by the lower surface area, a consequence of decreased PAHs, and thus, less soot nucleus, finally resulting in lower SVF in flames with higher strain rates.
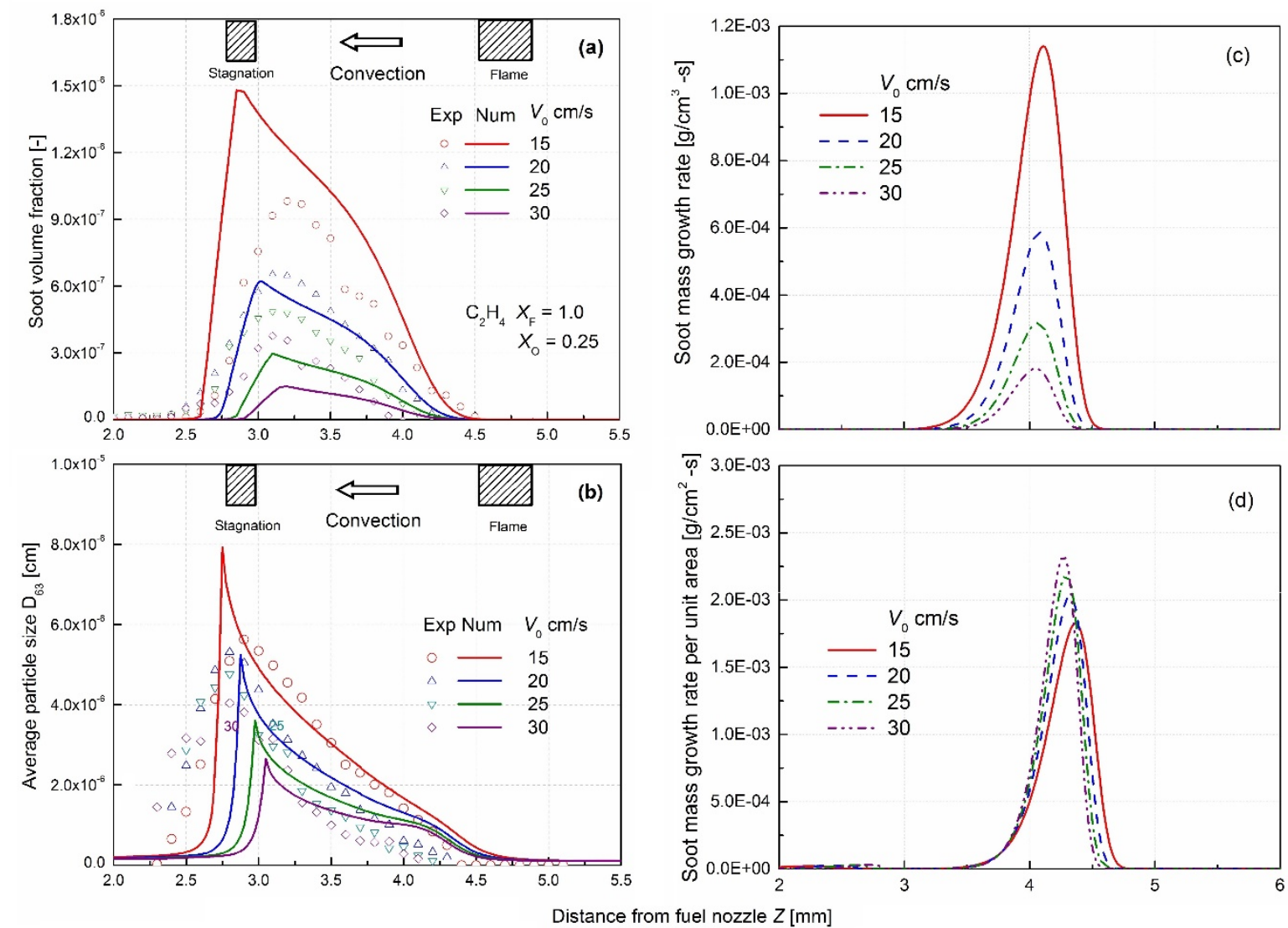

Figure 18. Effects of strain rate on axial profiles of soot volume fraction (a) average particle diameters, (b) soot mass growth rate, (c) and soot mass growth rate per unit area (d) in ethylene counterflow diffusion flames. Reprinted from Wang and Chung [91] with permission of Elsevier.

It is of interests to note here that the reduction of mass growth rate per unit surface area near the flame front, with an increase in straining, was also confirmed experimentally by Vandsburger et al. 
[554]. In particular, the complementary measurement of the velocity profiles using LDV allowed the authors to determine the time derivative of soot concentrations, which-after being normalized by the total soot surface area as estimated from measured soot sizes-gives the soot surface growth rate.

Wang and Chung [91] concluded that the reduction of soot formation with increasing strain rate can be generally explained as: 1) reduced PAH concentration and soot inception rate, leading to less soot surface area per unit volume for mass growth to occur; (2) reduced soot surface growth rate per unit volume; and (3) reduced residence time (from inception region to particle stagnation plane) available for soot surface reactions to take place.

Before closing this subsection, we point out that for an originally-sooting CDF, progressively higher strain rates (shorter residence times) sequentially lead to the disappearance of soot, PAH and finally, extinction of the flame [551, 555]. In fact, the critical strain rate at which soot starts to disappear $\left(K_{\mathrm{cr}}\right)$ is frequently used as a quantitative indicator of the sooting tendency of CDFs.

\subsection{Sooting structures of partially-premixed CDFs}

In a counterflow configuration, various flame interaction modes can be realized, including single, double and triple flames [556-559], depending on the degrees of partial premixing in the fuel and/or oxidizer streams. Partially-premixed flames are especially interesting when it comes to soot formation, since the existence of oxygen in the fuel stream can notably influence soot formation through its effects on both flame structures and soot chemistry.

Mungekar and Atreya [560-562] investigated the effects of partial premixing of oxygen in the fuel stream on the sooting structures in methane CDFs for both SF and SFO configurations. Several interesting features were reported for SF flames [562]. First, all the partially-premixed flames investigated featured double flames, with the sooting zones sandwiched between the premixed (PF) and non-premixed (NP) reaction zones, as schematically shown in Figure 19 (b-d). Second, with an increase in the level of premixing (as the equivalence ratio in the fuel stream $\phi$ decreased from infinity 
to $\phi=3.4$ ), the diffusion flame front moved closer to the gas stagnation plane (marked as SP), because of fuel dilution and consumption by oxygen. In addition, the separation distance between the premixed and non-premixed flame front increased. This is because the rich premixed flame sheet tended to move to the fuel nozzle with the addition of oxygen, which can be explained by considering the balance between the stretched laminar flame speed and the incoming reactant velocity [563]. In particular, the flame speed of the premixture was increased due to oxygen addition so that the premixed flame sheet adjusted itself to a location with higher incoming reactant speed (i.e., closer to the fuel boundary). Third, the peak soot volume fraction $\left(f_{\mathrm{v}}\right)$ and the soot zone thickness decreased and then increased again with the increase in the level of partial premixing. As explained by the authors $[561,562]$, the initial decrease in soot zone thickness (from $\phi=\infty$ to 4.5 ) was due to the shift of the diffusion flame towards the gas stagnation plane; subsequent increase (from $\phi=4.5$ to 3.4 ) was the result of increased spatial distance between the premixed and diffusion flame front, in which the soot zone was sandwiched. As to the variation of soot volume fraction, the authors attributed the initial reduction of $f_{\mathrm{v}}$ to the dilution and chemical effects of oxygen addition. As more oxygen was added, however, the flame structure changed significantly because the location of peak temperature moved closer to the stagnation plane, resulting in increased temperature and longer soot residence time in the soot formation region. Consequently, $\operatorname{peak} f_{\mathrm{v}}$ increased again as $\phi$ decreased from 4.5 to 3.4. Compared to the case of $\phi=\infty$ (Figure 19a), a considerable amount of OH radicals existed in the soot zone for the partially-premixed case with $\phi=3.4$. This led to the different sooting behavior: The soot particles leaked out near the stagnation plane for case of $\phi=\infty$, while they became oxidized when passing through the $\mathrm{OH}$ zone for $\phi=3.4$.

For the SFO flames [561] with diffusion flame on the fuel side of the stagnation plane, partial premixing in the fuel side resulted in a monotonic reduction of SVF. These results again support the importance of flame structure changes on soot evolution. 
In this section only the effect of partial-premixing on overall sooting structures is addressed. More detailed discussions on how partial premixing affects soot chemistry and quantitative soot loading will be discussed in a later section, when it will be seen that the effects can be drastically different for flames with different fuels.
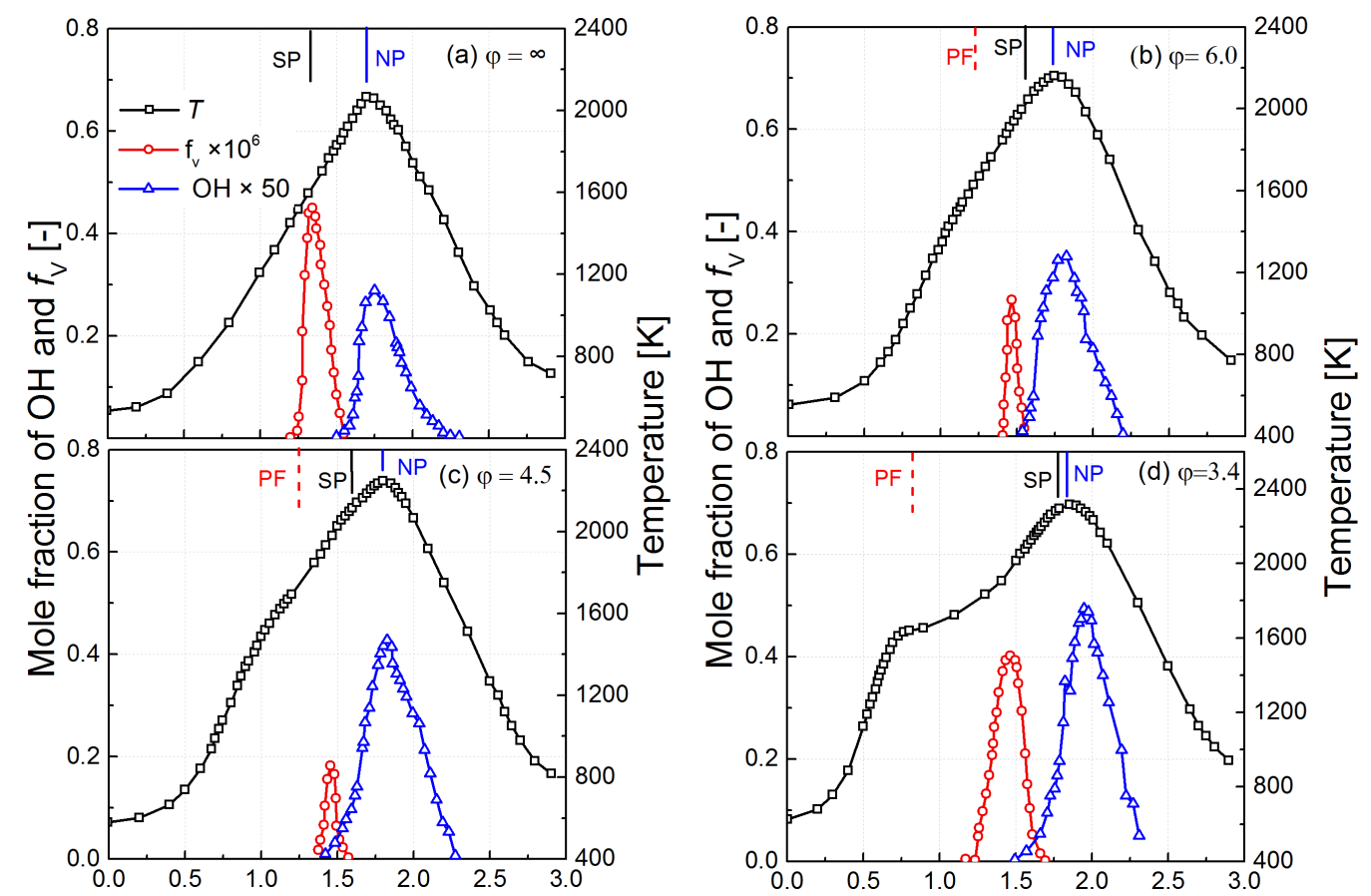

Distance from fuel nozzle $z[\mathrm{~cm}]$

Figure 19. Effects of oxygen addition on measured flame temperature, $\mathrm{OH}$, and soot volume fraction $f_{\mathrm{v}}$ for (a) purely diffusion $\mathrm{CH}_{4} \mathrm{CDF}$ (equivalence ratio in fuel stream $\phi=\infty$ ) and partially premixed cases with (b) $\phi=6.0$, (c) 4.5 and (d) 3.4 (PF: premixed flame, SP: stagnation plane, NP: non-premixed flame). Data taken from Mungekar and Atreya [562].

\section{Soot formation in counterflow diffusion flames: Parametric studies}

Next follows a thorough review of parametric soot studies performed in counterflow diffusion flames. Through a comprehensive search of the literature, more than 100 articles have been identified and classified into six categories, based on the research objectives of these studies. These studies generally focused on examining various factors that affect soot formation and include the effects of 1) fuel types, 2) dilution, 3) pressure, 4) temperature, 5) strain rate, and 6) other parameters such as 
radiation heat loss and electric field. For ease of reference Table 1 lists all relevant studies ordered by year of publication. For each individual study, fuel types, measured/predicted properties, diagnostic/numerical techniques, and the primary research objectives are listed. Note that several studies have more than one objective, such cases are grouped based on their primary objective of study, thus appearing only once in the list. Nevertheless, they can be referenced in different subsections in discussions that follow. Before examining the details, it is useful to summarize some general aspects of the factors that influence soot formation.

Generally, fuel molecular structures and additives determine pyrolysis products, thus affecting soot inception, surface growth and oxidation mainly through the influence on intermediate radical pools. As a controlling parameter for any kinetic process, temperature affects almost the entire soot formation process. Pressure and dilution influence soot inception and particle-particle coagulation by altering the concentration of important gaseous intermediates, as well as soot particles. Soot formation can also be significantly affected by flow field characteristics, primarily through their influence on flame structures, fuel/air mixing, strain rate and flame interactions. The electrical field may alter the flow field through ionic wind effects, also affecting soot formation, in addition to its potential role in altering the coagulation efficiency of charged soot particles. 
Table 1: Summary of soot studies performed in counterflow flow flames

\begin{tabular}{|c|c|c|c|}
\hline $\begin{array}{l}\text { Year/Author/ } \\
\text { Ref }\end{array}$ & $\begin{array}{l}\text { Research } \\
\text { Category }\end{array}$ & Fuel/Topic & Method \\
\hline $\begin{array}{l}1984, \\
\text { Vandsburger et } \\
\text { al. }[94]\end{array}$ & $\begin{array}{l}\text { Dilution } \\
\text { effects }\end{array}$ & $\begin{array}{l}\text { Fuel: Ethylene, propane } \\
\text { Topic: Effects of oxygen concentration in the } \\
\text { oxidizer stream on soot formation. }\end{array}$ & $\begin{array}{r}\text { EXP: LDV, } \\
\text { LE/LS }\end{array}$ \\
\hline $\begin{array}{l}1985, \\
\text { Vandsburger et } \\
\text { al. }[554]\end{array}$ & $\begin{array}{l}\text { Strain rate } \\
\text { effect }\end{array}$ & $\begin{array}{l}\text { Fuel: Ethylene } \\
\text { Topic: Effects of oxidizer inlet velocity on } \\
\text { soot formation of ethylene CDFs }\end{array}$ & EXP: LE/LS \\
\hline $\begin{array}{l}\text { 1987, Hura } \\
\text { and Glassman } \\
{[113]}\end{array}$ & $\begin{array}{l}\text { Partial } \\
\text { premixing }\end{array}$ & $\begin{array}{l}\text { Fuel: Ethylene, propene, propane, iso-butane, } \\
\text { n-butane } \\
\text { Topic:Effect of oxygen as additive to various } \\
\text { fuels on soot formation in CDF }\end{array}$ & $\begin{array}{l}\text { EXP: LDV, } \\
\text { LE/LS, LIF }\end{array}$ \\
\hline $\begin{array}{l}\text { 1988, } \\
\text { Axelbaum et } \\
\text { al. }[74]\end{array}$ & $\begin{array}{l}\text { Fuel dilution; } \\
\text { temperature } \\
\text { effects }\end{array}$ & $\begin{array}{l}\text { Fuel: Ethylene } \\
\text { Topic: Separation of effects of flame } \\
\text { temperature and fuel dilution on soot } \\
\text { formation }\end{array}$ & $\begin{array}{l}\text { EXP: LDV, } \\
\text { LE/LS }\end{array}$ \\
\hline $\begin{array}{l}\text { 1989, Du et al. } \\
\text { [551] }\end{array}$ & $\begin{array}{l}\text { Fuel dilution; } \\
\text { temperature } \\
\text { effects }\end{array}$ & $\begin{array}{l}\text { Fuel: Methane, ethane, propane, butane } \\
\text { Topic: Separation of flame temperature and } \\
\text { fuel dilution on soot formation for various } \\
\text { fuels }\end{array}$ & $\begin{array}{l}\text { EXP: LS, } \\
\text { LIF }\end{array}$ \\
\hline $\begin{array}{l}\text { 1991, Du et al. } \\
\text { [564] }\end{array}$ & $\begin{array}{l}\text { Non-HC } \\
\text { additive }\end{array}$ & $\begin{array}{l}\text { Fuel: Ethylene, propane } \\
\text { Topic: Effects of } \mathrm{CO}_{2} \text { and } \mathrm{O}_{2} \text { addition to fuel } \\
\text { and oxidizer side on soot formation }\end{array}$ & $\begin{array}{l}\text { EXP: LIF, } \\
\text { LDV, LE }\end{array}$ \\
\hline $\begin{array}{l}\text { 1991, Leung, } \\
\text { et al. [270] }\end{array}$ & $\begin{array}{l}\text { Soot } \\
\text { mechanism }\end{array}$ & $\begin{array}{l}\text { Fuel: Ethylene, propane } \\
\text { Topic: Phenomenological mechanism for soot } \\
\text { formation, tested for ethylene and propane } \\
\text { flames with varying oxygen concentrations in } \\
\text { air streams }\end{array}$ & SIM \\
\hline $\begin{array}{l}\text { 1993, Baranov } \\
\text { et al. [565] }\end{array}$ & Soot model & $\begin{array}{l}\text { Fuel: Acetylene } \\
\text { Topic: Soot model for spherical particles and } \\
\text { soot aggregates in low pressure acetylene } \\
\text { flames }\end{array}$ & SIM \\
\hline $\begin{array}{l}\text { 1993, Gomez } \\
\text { and Rosner } \\
\text { [79] }\end{array}$ & $\begin{array}{l}\text { Sooting } \\
\text { structure }\end{array}$ & $\begin{array}{l}\text { Fuel: Methane } \\
\text { Topic: Effect of thermophoresis on soot } \\
\text { characteristics in methane counterflow } \\
\text { diffusion methane flame }\end{array}$ & $\begin{array}{c}\text { EXP: LE/LS, } \\
\text { LDV }\end{array}$ \\
\hline $\begin{array}{l}\text { 1993, Taran } \\
{[566]}\end{array}$ & Electric field & $\begin{array}{l}\text { Fuel: Acetylene, benzene } \\
\text { Topic: Soot formation in low pressure } \\
\text { acetylene and benzene low flame subject to } \\
\text { alternating electric fields }\end{array}$ & EXP: TEM \\
\hline $\begin{array}{l}1994, \\
\text { Delichatsios } \\
{[567]}\end{array}$ & Soot model & $\begin{array}{l}\text { Fuel: Ethylene, butadiene, butene, acetylene } \\
\text { Topic: Phenomenological soot model for } \\
\text { prediction of soot formation. }\end{array}$ & SIM \\
\hline 1995, Du et al. & $\begin{array}{l}\text { Non-HC } \\
\text { additive }\end{array}$ & $\begin{array}{l}\text { Fuel: Ethylene, propane, butane } \\
\text { Topic: Effects of } \mathrm{CO}, \mathrm{Ar}, \mathrm{N}_{2}, \mathrm{He} \text {, and } \mathrm{H}_{2} \text { as } \\
\text { additives to ethylene, propane, and butane on } \\
\text { soot formation }\end{array}$ & $\begin{array}{c}\text { EXP: LS, } \\
\text { LIF; }\end{array}$ \\
\hline
\end{tabular}




\begin{tabular}{|c|c|c|c|}
\hline $\begin{array}{l}\text { 1995, Du et al. } \\
{[76]}\end{array}$ & $\begin{array}{l}\text { Sooting } \\
\text { structure }\end{array}$ & $\begin{array}{l}\text { Fuel: Ethylene } \\
\text { Topic: Effects of stoichiometric mixture } \\
\text { fraction on soot formation in counterflow and } \\
\text { coflow flames }\end{array}$ & $\begin{array}{l}\text { EXP: LDV } \\
\text { SIM }\end{array}$ \\
\hline $\begin{array}{l}\text { 1995, Yang } \\
\text { and Kennedy } \\
\text { [569] }\end{array}$ & Fuel additive & $\begin{array}{l}\text { Fuel: Methane (methyl chloride) } \\
\text { Topic: Effects of chlorine addition on soot } \\
\text { formation processes }\end{array}$ & $\begin{array}{r}\text { EXP: LDV, } \\
\text { LE/LS }\end{array}$ \\
\hline $\begin{array}{l}\text { 1996, Tseng, et } \\
\text { al. [570] }\end{array}$ & $\begin{array}{l}\text { Partial } \\
\text { premixing }\end{array}$ & $\begin{array}{l}\text { Fuel: Methane } \\
\text { Topic: Effects of oxygen addition in fuel on } \\
\text { formation of gaseous intermediate }\end{array}$ & $\begin{array}{l}\text { EXP: GC } \\
\text { SIM }\end{array}$ \\
\hline $\begin{array}{l}\text { 1996, } \\
\text { Marquardt et } \\
\text { al. }[571]\end{array}$ & $\begin{array}{l}\text { Sooting } \\
\text { process }\end{array}$ & $\begin{array}{l}\text { Fuel: Acetylene, carbon monoxide } \\
\text { Topic: Re-initiation of surface growth } \\
\text { reactions in premixed counterflow flames }\end{array}$ & EXP: LE/LS \\
\hline $\begin{array}{l}\text { 1996, Wang et } \\
\text { al. [78] }\end{array}$ & $\begin{array}{l}\text { Soot model; } \\
\text { strain rate } \\
\text { effects }\end{array}$ & $\begin{array}{l}\text { Fuel: Ethylene } \\
\text { Topic: PAH-based soot model to study } \\
\text { ethylene flames with different strain rates }\end{array}$ & $\begin{array}{l}\text { EXP: LDV, } \\
\text { LS/LE } \\
\text { SIM } \\
\end{array}$ \\
\hline $\begin{array}{l}\text { 1997, Kang et } \\
\text { al. [73] }\end{array}$ & $\begin{array}{l}\text { Sooting } \\
\text { structure }\end{array}$ & $\begin{array}{l}\text { Fuel: Propane } \\
\text { Topic: Effects of flow field on soot formation } \\
\text { in counterflow, flames. Classification of SF } \\
\text { and SFO flames. }\end{array}$ & $\begin{array}{l}\text { EXP: LDV, } \\
\text { LS/LE LIF }\end{array}$ \\
\hline $\begin{array}{l}1997, \\
\text { Vincitore and } \\
\text { Senkan [572] }\end{array}$ & $\begin{array}{l}\text { Flame } \\
\text { chemical } \\
\text { structure }\end{array}$ & $\begin{array}{l}\text { Fuel: Methane } \\
\text { Topic: Major intermediate species and soot } \\
\text { volume fraction profiles in methane CDF }\end{array}$ & $\begin{array}{l}\text { EXP: } \\
\text { GC/MS, } \\
\text { LE/LS }\end{array}$ \\
\hline $\begin{array}{l}\text { 1998, Chao et } \\
\text { al. [544] }\end{array}$ & $\begin{array}{l}\text { Sooting } \\
\text { structure }\end{array}$ & $\begin{array}{l}\text { Fuel: Ethylene } \\
\text { Topic: Effects of hydrodynamics and } \\
\text { transport characteristics on formation and } \\
\text { oxidation of soot/soot precursors formation } \\
\text { process }\end{array}$ & SIM \\
\hline $\begin{array}{l}\text { 1998, Du et al. } \\
\text { [573] }\end{array}$ & Pressure effect & $\begin{array}{l}\text { Fuel: Ethylene } \\
\text { Topic: Soot formation process in ethylene } \\
\text { flames at various pressure (from } 1 \text { to } 2.5 \mathrm{~atm} \text { ) }\end{array}$ & $\begin{array}{l}\text { EXP: LS, } \\
\text { LDV } \\
\text { SIM }\end{array}$ \\
\hline $\begin{array}{l}\text { 1998, Hwang } \\
\text { et al. }[165]\end{array}$ & $\begin{array}{l}\text { Partial } \\
\text { premixing }\end{array}$ & $\begin{array}{l}\text { Fuel: Ethylene, propane } \\
\text { Topic: Effects of propane and oxygen } \\
\text { addition soot formation in ethylene CDF }\end{array}$ & $\begin{array}{l}\text { EXP: LDV, } \\
\text { LE. LIF }\end{array}$ \\
\hline $\begin{array}{l}\text { 1998, Hwang } \\
\text { et al. [528] }\end{array}$ & Fuel mixing & $\begin{array}{l}\text { Fuel: Ethylene (propane) } \\
\text { Topic: Synergistic effect of soot formation } \\
\text { processes with ethylene-propane mixtures. }\end{array}$ & $\begin{array}{l}\text { EXP: LDV, } \\
\text { LS/LE, LIF; } \\
\text { SIM }\end{array}$ \\
\hline $\begin{array}{l}\text { 1998, Sung et } \\
\text { al. [85] }\end{array}$ & Pressure effect & $\begin{array}{l}\text { Fuel: Methane, propane } \\
\text { Topic: Sooting limits in methane and propane } \\
\text { flames with pressures from } 1 \text { to } 5 \mathrm{~atm}\end{array}$ & $\begin{array}{l}\text { EXP: Raman } \\
\text { SIM }\end{array}$ \\
\hline $\begin{array}{l}1998, \\
\text { Vincitore and } \\
\text { Senkan [242] }\end{array}$ & $\begin{array}{l}\text { Flame } \\
\text { chemical } \\
\text { structure; } \\
\text { Fuel dilution }\end{array}$ & $\begin{array}{l}\text { Fuel: Ethane } \\
\text { Topic: Chemical (PAH in particular) } \\
\text { speciation in ethane CDF with fuel dilution }\end{array}$ & $\begin{array}{l}\text { EXP: } \\
\text { GC/MS }\end{array}$ \\
\hline $\begin{array}{l}\text { 1998, Olten } \\
\text { and Senkan } \\
{[407]}\end{array}$ & $\begin{array}{l}\text { Flame } \\
\text { chemical } \\
\text { structure }\end{array}$ & $\begin{array}{l}\text { Fuel: Ethylene } \\
\text { Topic: Chemical speciation intermediates in } \\
\text { ethylene counterflow flame }\end{array}$ & $\begin{array}{l}\text { EXP: } \\
\text { GC/MS }\end{array}$ \\
\hline
\end{tabular}




\begin{tabular}{|c|c|c|c|}
\hline $\begin{array}{l}\text { 1999, Rubino } \\
\text { and Thomson } \\
\text { [574] }\end{array}$ & Fuel mixing & $\begin{array}{l}\text { Fuel: Propane (DMC, ethanol) } \\
\text { Topic: Effects of oxygenated additives (DMC } \\
\text { and ethanol) on soot precursor formation }\end{array}$ & EXP: GC \\
\hline $\begin{array}{l}\text { 2000, Böhm } \\
\text { and Lacas } \\
{[575]}\end{array}$ & Pressure effect & $\begin{array}{l}\text { Fuel: Methane } \\
\text { Topic: Effect of pressure ( } 1 \text { to } 6 \text { bar) on } \\
\text { extinction limits and PAH formation in } \\
\text { methane flames }\end{array}$ & $\begin{array}{l}\text { EXP: UV } \\
\text { LIF, } \\
\text { SIM }\end{array}$ \\
\hline $\begin{array}{l}\text { 2000, Leusden } \\
\text { and Peters [75] }\end{array}$ & $\begin{array}{l}\text { Partial } \\
\text { premixing }\end{array}$ & $\begin{array}{l}\text { Fuel: Acetylene } \\
\text { Topic: Effects of oxygen addition to fuel and } \\
\text { oxidizer on soot formation in acetylene } \\
\text { flames }\end{array}$ & $\begin{array}{l}\text { EXP: LE } \\
\text { SIM }\end{array}$ \\
\hline $\begin{array}{l}\text { 2000, Olten } \\
\text { and Senkan } \\
\text { [526] }\end{array}$ & $\begin{array}{l}\text { Flame } \\
\text { chemical } \\
\text { structure }\end{array}$ & $\begin{array}{l}\text { Fuel: Ethylene } \\
\text { Topic: Intermediate species profiles in } \\
\text { ethylene CDF. }\end{array}$ & $\begin{array}{l}\text { EXP: } \\
\text { GC/MS }\end{array}$ \\
\hline $\begin{array}{l}\text { 2001, Beltrame } \\
\text { et al. [576] }\end{array}$ & $\begin{array}{l}\text { Dilution and } \\
\text { strain rate } \\
\text { effect }\end{array}$ & $\begin{array}{l}\text { Fuel: Methane } \\
\text { Topic: Soot and NO formation in methane } \\
\text { flames with various oxygen contents and } \\
\text { strain rates }\left(10-60 \mathrm{~s}^{-1}\right)\end{array}$ & $\begin{array}{l}\text { EXP: LE } \\
\text { SIM }\end{array}$ \\
\hline $\begin{array}{l}\text { 2001, Böhm et } \\
\text { al. [114] }\end{array}$ & $\begin{array}{l}\text { Strain rate } \\
\text { effect }\end{array}$ & $\begin{array}{l}\text { Fuel: Methane } \\
\text { Topic: Soot precursor formation in methane } \\
\text { CDF flames with various strain rates }\end{array}$ & EXP: LIF \\
\hline $\begin{array}{l}\text { 2001, Hwang } \\
\text { and Chung } \\
{[281]}\end{array}$ & $\begin{array}{l}\text { Sooting } \\
\text { structure }\end{array}$ & $\begin{array}{l}\text { Fuel: Ethylene } \\
\text { Topic: Soot growth rates in ethylene CDF and } \\
\text { influencing factors }\end{array}$ & $\begin{array}{l}\text { EXP: LDV, } \\
\text { LE/LS, LIF } \\
\text { SIM }\end{array}$ \\
\hline $\begin{array}{l}2001, \\
\text { Santoianni et } \\
\text { al. }[577]\end{array}$ & $\begin{array}{l}\text { Strain rate } \\
\text { effect }\end{array}$ & $\begin{array}{l}\text { Fuel: Propane, methane } \\
\text { Topic: Soot formation in propane and } \\
\text { methane flames subject to oscillating strain } \\
\text { rates }\end{array}$ & $\begin{array}{l}\text { EXP: LII } \\
\text { PLIF, LDV, } \\
\text { PIV }\end{array}$ \\
\hline $\begin{array}{l}\text { 2002, Granata } \\
\text { et al. }[578]\end{array}$ & $\begin{array}{l}\text { Flame } \\
\text { chemical } \\
\text { structure }\end{array}$ & $\begin{array}{l}\text { Fuel:1,3-butadiene } \\
\text { Topic: Chemical speciation and kinetic } \\
\text { modelling with emphasis on soot and PAH } \\
\text { formation reaction pathways in 1,3-butadiene } \\
\text { flame }\end{array}$ & $\begin{array}{l}\text { EXP: } \\
\text { GC/MS } \\
\text { SIM }\end{array}$ \\
\hline $\begin{array}{l}\text { 2002, Naik and } \\
\text { Laurendeau } \\
\text { [579] }\end{array}$ & $\begin{array}{l}\text { Soot /NOx } \\
\text { interaction }\end{array}$ & $\begin{array}{l}\text { Fuel: Methane } \\
\text { Topic: Effect of radiation heat loss from soot } \\
\text { particles on nitric oxide formation }\end{array}$ & EXP: LIF \\
\hline $\begin{array}{l}\text { 2002, Pels } \\
\text { Leusden } \text { et al. } \\
\text { [580] }\end{array}$ & $\begin{array}{l}\text { Sooting } \\
\text { structure }\end{array}$ & $\begin{array}{l}\text { Fuel: Acetylene } \\
\text { Topic: Influence of aggregate formation on } \\
\text { soot growth process }\end{array}$ & $\begin{array}{l}\text { EXP: LE/LS } \\
\text { SIM }\end{array}$ \\
\hline $\begin{array}{l}\text { 2003, Naik et } \\
\text { al. [581] }\end{array}$ & $\begin{array}{l}\text { Dilution } \\
\text { effects }\end{array}$ & $\begin{array}{l}\text { Fuel: Methane } \\
\text { Topic: Critical sooting conditions in terms of } \\
\text { fuel/oxidizer dilution level }\end{array}$ & $\begin{array}{l}\text { EXP: LIF } \\
\text { SIM }\end{array}$ \\
\hline $\begin{array}{l}\text { 2003, Violi et } \\
\text { al. [87] }\end{array}$ & Soot model & $\begin{array}{l}\text { Fuel: Ethylene } \\
\text { Topic: PAH-based kinetic modelling of soot } \\
\text { formation in various ethylene diffusion flames }\end{array}$ & SIM \\
\hline $\begin{array}{l}\text { 2004, Lee et } \\
\text { al. [582] }\end{array}$ & Fuel mixing & $\begin{array}{l}\text { Fuel: Ethylene (propane, benzene) } \\
\text { Topic: Synergistic effect of ethylene-propane } \\
\text { mixtures and the effect of benzene addition on } \\
\text { soot formation }\end{array}$ & $\begin{array}{l}\text { EXP: LII, } \\
\text { PLIF }\end{array}$ \\
\hline
\end{tabular}




\begin{tabular}{|c|c|c|c|}
\hline $\begin{array}{l}\text { 2004, Liu et al. } \\
\text { [583] }\end{array}$ & Soot radiation & $\begin{array}{l}\text { Fuel: Ethylene } \\
\text { Topic: Effects of radiation heat loss on soot } \\
\text { formation }\end{array}$ & SIM \\
\hline $\begin{array}{l}\text { 2005, Berta et } \\
\text { al. [584] }\end{array}$ & $\begin{array}{l}\text { Partial } \\
\text { premixing }\end{array}$ & $\begin{array}{l}\text { Fuel: } n \text {-heptane } \\
\text { Topic: Effect of level of premixing on soot } \\
\text { formation }\end{array}$ & $\begin{array}{l}\text { EXP: GC } \\
\text { SIM: }\end{array}$ \\
\hline $\begin{array}{l}\text { 2005, Kitajima } \\
\text { et al. }[541]\end{array}$ & $\begin{array}{l}\text { Dilution effect; } \\
\text { strain rate } \\
\text { effect; } \\
\text { temperature } \\
\text { effects }\end{array}$ & $\begin{array}{l}\text { Fuel: Methane, propane } \\
\text { Topic: Effects of the dilution levels, flame } \\
\text { temperature, and strain rates on PAH } \\
\text { formation in methane and propane flames }\end{array}$ & $\begin{array}{l}\text { EXP: } \\
\text { GC/MS, } \\
\text { LDV }\end{array}$ \\
\hline $\begin{array}{l}2005, \\
\text { McNesby et al. } \\
{[101]}\end{array}$ & Fuel mixing & $\begin{array}{l}\text { Fuel: Ethylene } \\
\text { Topic: Effect of ethanol addition on } \\
\text { intermediate species and soot concentration }\end{array}$ & $\begin{array}{l}\text { EXP: PLIF, } \\
\text { LS } \\
\text { SIM }\end{array}$ \\
\hline $\begin{array}{l}\text { 2005, Xiao et } \\
\text { al. }[585]\end{array}$ & $\begin{array}{l}\text { Strain rate } \\
\text { effects }\end{array}$ & $\begin{array}{l}\text { Fuel: Methane, propane } \\
\text { Topic: Effect of strain rate on PAH and soot } \\
\text { formation in steady and unsteady CDFs }\end{array}$ & EXP; PLIF \\
\hline $\begin{array}{l}\text { 2005, Yoon et } \\
\text { al. }[586]\end{array}$ & Fuel mixing & $\begin{array}{l}\text { Fuel: Ethylene } \\
\text { Topic: Kinetic effects of methane, ethane, } \\
\text { propane, and propene) mixing on PAH and } \\
\text { soot formation }\end{array}$ & $\begin{array}{l}\text { EXP: PLII, } \\
\text { LIF } \\
\text { SIM: }\end{array}$ \\
\hline $\begin{array}{l}\text { 2006, Berta et } \\
\text { al. [563] }\end{array}$ & $\begin{array}{l}\text { Partial } \\
\text { premixing }\end{array}$ & $\begin{array}{l}\text { Fuel: } n \text {-heptane } \\
\text { Topic: } \mathrm{NO}_{\mathrm{x}} \text { and } \mathrm{PAH} \text { formation in n-heptane } \\
\text { counter-flow diffusion flame with various } \\
\text { partial premixing levels }\end{array}$ & $\begin{array}{l}\text { EXP: GC } \\
\text { SIM: }\end{array}$ \\
\hline $\begin{array}{l}2006, \\
\text { Mungekar and } \\
\text { Atreya [561] }\end{array}$ & $\begin{array}{l}\text { Partial } \\
\text { premixing }\end{array}$ & $\begin{array}{l}\text { Fuel: Methane } \\
\text { Topic: Effects of premixing level on radiation } \\
\text { and sooting characteristics }\end{array}$ & $\begin{array}{l}\text { EXP: LE/LS, } \\
\text { TEM, LIF } \\
\text { SIM }\end{array}$ \\
\hline $\begin{array}{l}2006, \\
\text { Mungekar and } \\
\text { Atreya [562] }\end{array}$ & $\begin{array}{l}\text { Partial } \\
\text { premixing }\end{array}$ & $\begin{array}{l}\text { Fuel: Methane } \\
\text { Topic: Flame radiation and soot formation in } \\
\text { methane flames with fuel-side oxygen } \\
\text { addition }\end{array}$ & $\begin{array}{l}\text { EXP: TEM, } \\
\text { LIF, LE/LS } \\
\text { SIM }\end{array}$ \\
\hline $\begin{array}{l}\text { 2007, Chung } \\
\text { and Violi [380] }\end{array}$ & Soot model & $\begin{array}{l}\text { Fuel: Propene } \\
\text { Topic: Importance of oxidative reactions on } \\
\text { soot growth and the dependence of soot } \\
\text { structure on combustion conditions }\end{array}$ & SIM \\
\hline $\begin{array}{l}\text { 2007, Humer } \\
\text { et al. [587] }\end{array}$ & Fuel mixing & $\begin{array}{l}\text { Fuel: n-Decane, n-Dodecane, Methyl-cyclo- } \\
\text { hexane, Toluene, o-Xylene } \\
\text { Topic: Fuel combustion properties of } \\
\text { surrogate fuels. }\end{array}$ & $\begin{array}{l}\text { EXP: Visual } \\
\text { flame } \\
\text { appearance } \\
\text { SIM }\end{array}$ \\
\hline $\begin{array}{l}\text { 2007, Li et al. } \\
{[588]}\end{array}$ & $\begin{array}{l}\text { Carbon } \\
\text { material } \\
\text { synthesis }\end{array}$ & $\begin{array}{l}\text { Fuel: Methane } \\
\text { Topic: Effects of catalyst, temperature, and } \\
\text { strain rate of the flow on carbon nanotubes } \\
\text { synthesis. }\end{array}$ & EXP: SEM \\
\hline $\begin{array}{l}\text { 2007, Sarathy } \\
\text { et al. [589] }\end{array}$ & Fuel type & $\begin{array}{l}\text { Fuel: Methyl- crotonate, methyl-butanoate } \\
\text { Topic: Effects of the FAME molecular } \\
\text { structure on the formation of intermediate } \\
\text { species. }\end{array}$ & EXP: GC \\
\hline
\end{tabular}




\begin{tabular}{|c|c|c|c|}
\hline $\begin{array}{l}\text { 2007, } \\
\text { Yamamoto et } \\
\text { al. [590], }\end{array}$ & $\begin{array}{l}\text { Strain rate } \\
\text { effects }\end{array}$ & $\begin{array}{l}\text { Fuel: Acetylene } \\
\text { Topic: Effects of strain rate on PAH } \\
\text { formation in CDFs }\end{array}$ & $\begin{array}{l}\text { EXP: } \\
\text { GC/MS }\end{array}$ \\
\hline $\begin{array}{l}\text { 2007, Yoo and } \\
\operatorname{Im}[591]\end{array}$ & $\begin{array}{l}\text { Sooting } \\
\text { structure }\end{array}$ & $\begin{array}{l}\text { Fuel: Ethylene } \\
\text { Topic: Soot dynamics in turbulent counter- } \\
\text { flow flames }\end{array}$ & SIM \\
\hline $\begin{array}{l}\text { 2008, Berta et } \\
\text { al. [592] }\end{array}$ & $\begin{array}{l}\text { Flame } \\
\text { chemical } \\
\text { structure }\end{array}$ & $\begin{array}{l}\text { Fuel: n-Heptane } \\
\text { Topic: Formation pathways for aromatic } \\
\text { species. }\end{array}$ & $\begin{array}{l}\text { EXP: GC } \\
\text { SIM }\end{array}$ \\
\hline $\begin{array}{l}\text { 2008, Cuoci et } \\
\text { al. }[593]\end{array}$ & $\begin{array}{l}\text { Strain rate } \\
\text { effects }\end{array}$ & $\begin{array}{l}\text { Fuel: Methane } \\
\text { Topic: Frequency response of PAH } \\
\text { concentration to strain rate harmonic } \\
\text { oscillations }\end{array}$ & SIM \\
\hline $\begin{array}{l}2008, \\
\text { Leylegian } \\
{[594]}\end{array}$ & Fuel mixing & $\begin{array}{l}\text { Fuel: Methane, Elethylene } \\
\text { Topic: Effects of chloromethane addition on } \\
\text { soot formation }\end{array}$ & $\begin{array}{l}\text { EXP: LDV, } \\
\text { LS } \\
\text { SIM: }\end{array}$ \\
\hline $\begin{array}{l}\text { 2008, Yoon et } \\
\text { al. }[100]\end{array}$ & Fuel mixing & $\begin{array}{l}\text { Fuel: Methane, ethane, propane, ethylene } \\
\text { Topic: Effect of the addition of dimethyl ether } \\
\text { on polycyclic aromatic hydrocarbon and soot } \\
\text { formation }\end{array}$ & $\begin{array}{l}\text { EXP: LII, } \\
\text { LIF } \\
\text { SIM: }\end{array}$ \\
\hline $\begin{array}{l}\text { 2008, Zhong } \\
\text { and } \mathrm{Xi}[595]\end{array}$ & $\begin{array}{l}\text { Kinetic } \\
\text { mechanism }\end{array}$ & $\begin{array}{l}\text { Fuel: } n \text {-heptane } \\
\text { Topic: Reduction of a detailed n-heptane } \\
\text { chemical kinetic mechanism (validated in } \\
\text { CDF) }\end{array}$ & SIM \\
\hline $\begin{array}{l}2009, \\
\text { Blanquart et } \\
\text { al. [596] }\end{array}$ & $\begin{array}{l}\text { Kinetic } \\
\text { mechanism }\end{array}$ & $\begin{array}{l}\text { Fuel: Acetylene, n-heptane } \\
\text { Topic: Development of a kinetic model with } \\
\text { PAH formation (validated in CDF) }\end{array}$ & SIM \\
\hline $\begin{array}{l}\text { Blanquart and } \\
\text { Pitsch [597] }\end{array}$ & Soot model & $\begin{array}{l}\text { Fuel: Acetylene } \\
\text { Topic: Development of a new PAH-based } \\
\text { soot model using method of moments. }\end{array}$ & SIM \\
\hline $\begin{array}{l}\text { 2009, Cuoci et } \\
\text { al. [395] }\end{array}$ & $\begin{array}{l}\text { Strain rate } \\
\text { effects }\end{array}$ & $\begin{array}{l}\text { Fuel: Propane } \\
\text { Topic: Response of soot volume fraction to } \\
\text { strain rate oscillations }\end{array}$ & SIM \\
\hline $\begin{array}{l}\text { 2009, Cuoci et } \\
\text { al. }[598]\end{array}$ & $\begin{array}{l}\text { Strain rate } \\
\text { effects }\end{array}$ & $\begin{array}{l}\text { Fuel: Methane, propane, ethylene } \\
\text { Topic: PAH and soot formation in unsteady } \\
\text { counterflow diffusion flames with strain rate } \\
\text { oscillations }\end{array}$ & SIM \\
\hline $\begin{array}{l}\text { 2009, D’Anna } \\
\text { et al. [531] }\end{array}$ & $\begin{array}{l}\text { Sooting } \\
\text { structure; } \\
\text { soot model }\end{array}$ & $\begin{array}{l}\text { Fuel: Ethylene } \\
\text { Topic: Soot growth and evolution in ethylene } \\
\text { CDF. }\end{array}$ & $\begin{array}{l}\text { EXP: LII, } \\
\text { LIF, } \\
\text { SIM }\end{array}$ \\
\hline $\begin{array}{l}\text { 2009, Hou et } \\
\text { al. [599] }\end{array}$ & $\begin{array}{l}\text { Carbon } \\
\text { material } \\
\text { synthesis }\end{array}$ & $\begin{array}{l}\text { Fuel: Ethylene, methane } \\
\text { Topic: Effects of fuel/oxygen concentration } \\
\text { on t carbon nano-onions formation }\end{array}$ & $\begin{array}{l}\text { EXP: SEM, } \\
\text { TEM }\end{array}$ \\
\hline $\begin{array}{l}2009, \\
\text { Jahangirian } \\
{[80]}\end{array}$ & Fuel mixing & $\begin{array}{l}\text { Fuel: Ethylene } \\
\text { Topic: Soot precursor formation in ethylene } \\
\text { CDF doped with jet surrogate fuels }\end{array}$ & $\begin{array}{l}\text { EXP: } \\
\text { GC/MS }\end{array}$ \\
\hline $\begin{array}{l}\text { 2009, Mehta et } \\
\text { al. }[600]\end{array}$ & Soot model & $\begin{array}{l}\text { Fuel: Ethylene } \\
\text { Topic: Sensitivity of soot model parameters } \\
\text { in modeling soot in CDF. }\end{array}$ & SIM \\
\hline
\end{tabular}




\begin{tabular}{|c|c|c|c|}
\hline $\begin{array}{l}2010 . \\
\text { Frassoldati et } \\
\text { al. }[601]\end{array}$ & Fuel type & $\begin{array}{l}\text { Fuel: n-propanol, iso-propanol } \\
\text { Topic: Chemical speciation and kinetic } \\
\text { analysis of n-propanol and iso-propanol } \\
\text { flames, with discussions on soot precursors. }\end{array}$ & $\begin{array}{l}\text { EXP: } \\
\text { GC/MS } \\
\text { SIM }\end{array}$ \\
\hline $\begin{array}{l}\text { 2010, Karatas } \\
\text { et al. }[86]\end{array}$ & Fuel mixing & $\begin{array}{l}\text { Fuel: n-butane, iso-butane, ethylene, propane, } \\
\text { Topic: Synergistic effect of fuel mixture on } \\
\text { soot formation }\end{array}$ & EXP: \\
\hline $\begin{array}{l}\text { 2011, Choi et } \\
\text { al. [447] }\end{array}$ & Fuel mixing & $\begin{array}{l}\text { Fuel: n-heptane, iso-octane, toluene } \\
\text { Topic: Synergistic effect on PAH formation } \\
\text { for n-heptane/toluene and iso-octane/toluene } \\
\text { mixtures }\end{array}$ & $\begin{array}{l}\text { EXP: LIF, } \\
\text { LII } \\
\text { SIM }\end{array}$ \\
\hline $\begin{array}{l}\text { 2011, Choi et } \\
\text { al. [602] }\end{array}$ & Fuel mixing & $\begin{array}{l}\text { Fuel: Ethylene } \\
\text { Topic: Kinetic insights on the effects of } \\
\text { methane, ethane, or propane addition in } \\
\text { ethylene fuels on soot formation }\end{array}$ & $\begin{array}{l}\text { EXP: LE/LS } \\
\text { SIM }\end{array}$ \\
\hline $\begin{array}{l}\text { 2011, Hayashi } \\
\text { et al. [603] }\end{array}$ & Spray flame & $\begin{array}{l}\text { Fuel: } n \text {-decane } \\
\text { Topic: Effects of fuel droplet size on soot } \\
\text { formation in spray flames }\end{array}$ & $\begin{array}{l}\text { EXP: LII, } \\
\text { TR-LII; } \\
\text { SIM }\end{array}$ \\
\hline $\begin{array}{l}2011, \\
\text { Narayanan et } \\
\text { al. }[604]\end{array}$ & Soot radiation & $\begin{array}{l}\text { Fuel: Ethylene } \\
\text { Topic: Effect of radiation heat loss from soot } \\
\text { particles on flame extinction }\end{array}$ & SIM \\
\hline $\begin{array}{l}\text { 2011, Seiser et } \\
\text { al. [605] }\end{array}$ & Fuel type & $\begin{array}{l}\text { Fuel: } n \text {-decane, JP-10 } \\
\text { Topic: Chemical speciation and kinetic } \\
\text { modelling of n-decane and JP-10 flames, with } \\
\text { discussions on soot precursors }\end{array}$ & $\begin{array}{l}\text { EXP: GC } \\
\text { SIM }\end{array}$ \\
\hline $\begin{array}{l}\text { 2012, Carbone } \\
\text { and Gomez } \\
{[606]}\end{array}$ & Fuel mixing & $\begin{array}{l}\text { Fuel: Methane, ethylene (toluene) } \\
\text { Topic: Chemical structures of toluene-doped } \\
\text { methane flame and ethylene flame }\end{array}$ & $\begin{array}{l}\text { EXP: } \\
\text { GC/MS } \\
\text { SIM }\end{array}$ \\
\hline $\begin{array}{l}\text { 2012, Feng et } \\
\text { al. [82] }\end{array}$ & Fuel type & $\begin{array}{l}\text { Fuel: Biodiesel } \\
\text { Topic: Sooting tendencies of various } \\
\text { biodiesel fuels }\end{array}$ & $\begin{array}{l}\text { EXP: } \\
\text { GC/MS, LE } \\
\text { SIM }\end{array}$ \\
\hline $\begin{array}{l}\text { 2012, Raj et al. } \\
\text { [203] }\end{array}$ & $\begin{array}{l}\text { Kinetic } \\
\text { mechanism }\end{array}$ & $\begin{array}{l}\text { Fuel: iso-octane, } n \text {-heptane, toluene } \\
\text { Topic: Mechanism development for gasoline } \\
\text { surrogate fuels with an emphasis on the } \\
\text { formation of large PAHs }\end{array}$ & SIM \\
\hline $\begin{array}{l}2012, \\
\text { Salamanca et } \\
\text { al. }[607]\end{array}$ & Fuel mixing & $\begin{array}{l}\text { Fuel: Ethylene } \\
\text { Topic: Effect of ethanol addition on } \\
\text { particulate formation in ethylene flames }\end{array}$ & $\begin{array}{l}\text { EXP: LIF, } \\
\text { LII, TR-LIE }\end{array}$ \\
\hline $\begin{array}{l}2012, \\
\text { Sirignano et al. } \\
{[608]}\end{array}$ & $\begin{array}{l}\text { Sooting } \\
\text { structure }\end{array}$ & $\begin{array}{l}\text { Fuel: Ethylene } \\
\text { Topic: Demonstrating the correlation of } \\
\text { fluorescence emission spectra and } \\
\text { fluorescence lifetimes with the characteristics } \\
\text { of the PAH/incipient soot particles }\end{array}$ & $\begin{array}{l}\text { EXP: TR- } \\
\text { LII, LIF }\end{array}$ \\
\hline $\begin{array}{l}2012, \\
\text { Slavinskaya et } \\
\text { al. [63] }\end{array}$ & $\begin{array}{l}\text { Kinetic } \\
\text { mechanism }\end{array}$ & $\begin{array}{l}\text { Fuel: Ethylene, ethane } \\
\text { Topic: Development of PAH growth chemical } \\
\text { kinetic mechanism with validation in CDF }\end{array}$ & SIM \\
\hline $\begin{array}{l}\text { 2013, Carbone } \\
\text { and Gomez } \\
{[609]}\end{array}$ & Fuel mixing & $\begin{array}{l}\text { Fuel: Methane, ethylene (1,2,4-trimethyl } \\
\text { benzene) } \\
\text { Topic: Chemical effect of 1,2,4-trimethyl } \\
\text { benzene addition in Methane/ethylene flames }\end{array}$ & $\begin{array}{l}\text { EXP: } \\
\text { GC/MS } \\
\text { SIM: }\end{array}$ \\
\hline
\end{tabular}




\begin{tabular}{|c|c|c|c|}
\hline $\begin{array}{l}\text { 2013, Fu et al. } \\
{[610]}\end{array}$ & Fuel type & $\begin{array}{l}\text { Fuel: } n \text {-heptane, } 1 \text {-heptene } \\
\text { Topic: Effects of unsaturated (double) bond } \\
\text { on PAH and soot emissions in partially } \\
\text { premixed flames }\end{array}$ & SIM \\
\hline $\begin{array}{l}\text { 2013, Han, et } \\
\text { al. [611] }\end{array}$ & Fuel type & $\begin{array}{l}\text { Fuel: } n \text {-heptane, 1-heptene } \\
\text { Topic: Effect of the unsaturated bond in the } \\
\text { fuel molecular structure on NOx and soot } \\
\text { emissions lean premixed, rich premixed, and } \\
\text { non-premixed combustion }\end{array}$ & SIM \\
\hline $\begin{array}{l}\text { 2013, Hayashi } \\
\text { et al. [612] }\end{array}$ & Spray flame & $\begin{array}{l}\text { Fuel: } n \text {-decane } \\
\text { Topic: Effects of fuel droplet size distribution } \\
\text { on soot formation }\end{array}$ & $\begin{array}{l}\text { EXP: LII; } \\
\text { SIM }\end{array}$ \\
\hline $\begin{array}{l}2013, \\
\text { Hernández et } \\
\text { al. }[613]\end{array}$ & Soot radiation & $\begin{array}{l}\text { Fuel: Ethylene } \\
\text { Topic: Effects of soot and gas radiation on } \\
\text { soot formation and flame structure }\end{array}$ & SIM \\
\hline 2013, Joo et al. & Fuel mixing & $\begin{array}{l}\text { Fuel: Ethylene, propane } \\
\text { Topic: Sooting limits of ethylene-propane } \\
\text { mixtures }\end{array}$ & EXP: LE/LS \\
\hline $\begin{array}{l}\text { 2013, Skeen et } \\
\text { al. [615] }\end{array}$ & $\begin{array}{l}\text { Soot chemical } \\
\text { properties }\end{array}$ & $\begin{array}{l}\text { Fuel: Acetylene, ethylene, propane } \\
\text { Topic: Chemical identification of species } \\
\text { condensed on soot particles }\end{array}$ & $\begin{array}{l}\text { EXP: } \\
\text { Aerosol } \\
\text { TOF-MS }\end{array}$ \\
\hline $\begin{array}{l}\text { 2013, Skeen et } \\
\text { al. [413] }\end{array}$ & $\begin{array}{l}\text { Pressure } \\
\text { effects }\end{array}$ & $\begin{array}{l}\text { Fuel: Acetylene } \\
\text { Topic: Chemical speciation composition of } \\
\text { acetylene flames at various pressures }\end{array}$ & $\begin{array}{l}\text { EXP: MBMS } \\
\text { SIM }\end{array}$ \\
\hline $\begin{array}{l}\text { 2013, Wang et } \\
\text { al. [616] }\end{array}$ & Fuel mixing & $\begin{array}{l}\text { Fuel: Ethylene } \\
\text { Topic: Kinetic insights on the effect of } \\
\text { mixing propane and benzene in the ethylene } \\
\text { flames }\end{array}$ & SIM \\
\hline $\begin{array}{l}\text { 2013, Xia et } \\
\text { al. [77] }\end{array}$ & $\begin{array}{l}\text { Sooting } \\
\text { structure }\end{array}$ & $\begin{array}{l}\text { Fuel: Ethylene, propane } \\
\text { Topic: utility of } C / O \text { ratio space in } \\
\text { interpreting the effect of } Z_{\text {st }} \text { on sooting } \\
\text { structure }\end{array}$ & SIM \\
\hline $\begin{array}{l}\text { 2014, Carbone } \\
\text { and Gomez } \\
{[617]}\end{array}$ & Fuel mixing & $\begin{array}{l}\text { Fuel: Methane, ethylene } \\
\text { Topic: Effects of the addition of n-decane in } \\
\text { the ethylene flame and ethylene flame on soot } \\
\text { precursor formation }\end{array}$ & $\begin{array}{l}\text { EXP: } \\
\text { GC/MS, } \\
\text { SIM }\end{array}$ \\
\hline $\begin{array}{l}\text { 2014, Figura } \\
\text { and Gomez } \\
{[618]}\end{array}$ & $\begin{array}{l}\text { Pressure } \\
\text { effects }\end{array}$ & $\begin{array}{l}\text { Fuel: Ethylene } \\
\text { Topic: Chemical structure of ethylene flames } \\
\text { at pressures up to } 2.5 \mathrm{Mpa}(0.1-2.5 \mathrm{Mpa}) \text { with } \\
\text { discussions on soot precursors }\end{array}$ & $\begin{array}{l}\text { EXP: } \\
\text { GC/MS }\end{array}$ \\
\hline $\begin{array}{l}\text { 2014, Park et } \\
\text { al. [527] }\end{array}$ & $\begin{array}{l}\text { Electrical } \\
\text { fields }\end{array}$ & $\begin{array}{l}\text { Fuel: Ethylene } \\
\text { Topic: Effect of DC electric field on soot } \\
\text { reduction }\end{array}$ & $\begin{array}{l}\text { EXP: PLII, } \\
\text { PLIF }\end{array}$ \\
\hline $\begin{array}{l}2014, \\
\text { Sirignano, et } \\
\text { al. }[619]\end{array}$ & Fuel mixing & $\begin{array}{l}\text { Fuel: Ethylene } \\
\text { Topic: Effect of dimethyl ether (DME) } \\
\text { addition on particulate formation }\end{array}$ & EXP: LIE, \\
\hline $\begin{array}{l}\text { 2014, Wang } \\
\text { and Chung } \\
{[620]}\end{array}$ & Fuel type & $\begin{array}{l}\text { Fuel: Methane, ethane, propane, n-butane, } \\
\text { ethylene, propene, iso-butane }\end{array}$ & EXP: LS/LE \\
\hline
\end{tabular}




\begin{tabular}{|c|c|c|c|}
\hline & & $\begin{array}{l}\text { Topic: Development of CDF-based } \\
\text { quantitative indices of sooting tendencies }\end{array}$ & \\
\hline $\begin{array}{l}\text { 2015, Carbone } \\
\text { et al. }[81]\end{array}$ & $\begin{array}{l}\text { Partial } \\
\text { premixing }\end{array}$ & $\begin{array}{l}\text { Fuel: Ethylene } \\
\text { Topic: Chemical structure of incipiently } \\
\text { sooting partially premixed ethylene flames }\end{array}$ & $\begin{array}{l}\text { EXP: } \\
\text { GC/MS } \\
\text { SIM: }\end{array}$ \\
\hline $\begin{array}{l}\text { 2015, Carbone } \\
\text { and Gomez } \\
{[621]}\end{array}$ & Fuel mixing & $\begin{array}{l}\text { Fuel: Methane, ethylene } \\
\text { Topic: Chemical interaction of n-decane and } \\
\text { 1,2,4-trimethyl benzene addition in methane } \\
\text { flame and ethylene flame }\end{array}$ & $\begin{array}{l}\text { EXP: } \\
\text { GC/MS } \\
\text { SIM }\end{array}$ \\
\hline $\begin{array}{l}\text { 2015, Choi et } \\
\text { al. }[476]\end{array}$ & Fuel mixing & $\begin{array}{l}\text { Fuel: Ethylene (DME) } \\
\text { Topic: Effect of DME mixing on number } \\
\text { density and size properties of soot particles in } \\
\text { ethylene flame }\end{array}$ & $\begin{array}{l}\text { EXP: LE/LS } \\
\text { SIM: }\end{array}$ \\
\hline $\begin{array}{l}\text { 2015, Choi et } \\
\text { al. [622] }\end{array}$ & Fuel mixing & $\begin{array}{l}\text { Fuel: Ethylene } \\
\text { Topic: Effect of n-heptane, toluene, benzene, } \\
\text { and ethanol addition to ethylene fuels on PAH } \\
\text { and soot formation }\end{array}$ & $\begin{array}{l}\text { EXP: LIF, } \\
\text { LII, } \\
\text { SIM }\end{array}$ \\
\hline $\begin{array}{l}\text { 2015, Figura et } \\
\text { al. [623] }\end{array}$ & $\begin{array}{l}\text { Pressure } \\
\text { effects }\end{array}$ & $\begin{array}{l}\text { Fuel: Ethylene } \\
\text { Topic: Experimental methods for probing } \\
\text { high pressure CDF }\end{array}$ & $\begin{array}{l}\text { EXP: } \\
\text { GC/MS }\end{array}$ \\
\hline $\begin{array}{l}2015, \\
\text { Johansson et } \\
\text { al. }[136]\end{array}$ & $\begin{array}{l}\text { Kinetic } \\
\text { pathway }\end{array}$ & $\begin{array}{l}\text { Fuel: Ethylene } \\
\text { Topic: Insights on the formation/growth } \\
\text { pathways for soot precursors (PAHs) }\end{array}$ & $\begin{array}{l}\text { EXP: TOF- } \\
\text { MS } \\
\text { SIM }\end{array}$ \\
\hline $\begin{array}{l}\text { 2015, Raj et al. } \\
\text { [261] }\end{array}$ & Fuel additive & $\begin{array}{l}\text { Fuel: Methane } \\
\text { Topic: Effects of } \mathrm{Fe}(\mathrm{CO}) 5 \text { addition on soot } \\
\text { precursors, } \mathrm{CO} \text { and NO formation }\end{array}$ & $\begin{array}{l}\text { EXP: GC, } \\
\text { FTIR, XRD }\end{array}$ \\
\hline $\begin{array}{l}\text { 2015, Schenk } \\
\text { et al. [208] }\end{array}$ & $\begin{array}{l}\text { Fuel type; } \\
\text { kinetic } \\
\text { pathway }\end{array}$ & $\begin{array}{l}\text { Fuel: n-butane, i-butane, i-butene, i-butanol } \\
\text { Topic: Experimental insights into the } \\
\text { chemistry of soot formation in flames of } \\
\text { different fuels }\end{array}$ & $\begin{array}{l}\text { EXP: } \\
\text { GC/MS, } \\
\text { Helium-ion } \\
\text { microscopy }\end{array}$ \\
\hline $\begin{array}{l}2015, \\
\text { Sirignano et al. } \\
{[372]}\end{array}$ & $\begin{array}{l}\text { Sooting } \\
\text { structure; } \\
\text { kinetic } \\
\text { pathway }\end{array}$ & $\begin{array}{l}\text { Fuel: Ethylene } \\
\text { Topic: Soot formation and oxidation in both } \\
\text { SF and SFO CDF }\end{array}$ & EXP: LII, LS \\
\hline $\begin{array}{l}\text { 2015, Wang et } \\
\text { al. }[140]\end{array}$ & $\begin{array}{l}\text { Soot model; } \\
\text { fuel mixing }\end{array}$ & $\begin{array}{l}\text { Fuel: Ethylene } \\
\text { Topic: Chemical cross-linking effect between } \\
\text { ethylene and the dopant fuels and effects on } \\
\text { PAH and soot formation }\end{array}$ & $\begin{array}{l}\text { EXP: LE/LS, } \\
\text { SIM }\end{array}$ \\
\hline $\begin{array}{l}\text { 2016, Choi et } \\
\text { al. [624] }\end{array}$ & $\begin{array}{l}\text { Non-HC } \\
\text { additive }\end{array}$ & $\begin{array}{l}\text { Fuel: Ethylene } \\
\text { Topic: Effects of hydrogen addition on micro } \\
\text { and nanostructure of soot particles in ethylene } \\
\text { flames }\end{array}$ & EXP: TEM \\
\hline $\begin{array}{l}\text { 2016, Selvaraj } \\
\text { et al. [625] }\end{array}$ & Soot model & $\begin{array}{l}\text { Fuel: Ethylene } \\
\text { Topic: Importance of accounting for large } \\
\text { PAH formation in soot models }\end{array}$ & SIM \\
\hline $\begin{array}{l}\text { 2016, Singh et } \\
\text { al. }[84]\end{array}$ & Fuel type & $\begin{array}{l}\text { Fuel: n-butane, iso-butane, n-butanol, iso- } \\
\text { butanol, sec-butanol, tert-butanol } \\
\text { Topic: Soot formation processes for flames of } \\
\text { butane and butanol isomers }\end{array}$ & $\begin{array}{l}\text { EXP: LII, LE } \\
\text { SIM }\end{array}$ \\
\hline
\end{tabular}




\begin{tabular}{|c|c|c|c|}
\hline $\begin{array}{l}\text { 2016, Singh } \\
\text { and Sung [441] }\end{array}$ & $\begin{array}{l}\text { Fuel type; } \\
\text { Fuel mixing }\end{array}$ & $\begin{array}{l}\text { Fuel: n-butane, iso-butane, n-butanol, iso- } \\
\text { butanol, sec-butanol, tert-butanol } \\
\text { Topic: PAHs and soot formation in flames of } \\
\text { isomeric fuels with kinetic analysis }\end{array}$ & $\begin{array}{l}\text { EXP: PLIF, } \\
\text { LII, LE } \\
\text { SIM }\end{array}$ \\
\hline $\begin{array}{l}\text { 2016, Wang } \\
\text { and Chung } \\
\text { [91] }\end{array}$ & $\begin{array}{l}\text { Strain rate } \\
\text { effects; } \\
\text { dilution } \\
\text { effects; } \\
\text { fuel type }\end{array}$ & $\begin{array}{l}\text { Fuel: Ethylene, ethane, propylene, propane } \\
\text { Topic: Effect of strain rate, oxygen } \\
\text { enrichment and fuel type on the sooting } \\
\text { characteristics in CDF }\end{array}$ & $\begin{array}{l}\text { EXP: LII, } \\
\text { LIF, LE/LS }\end{array}$ \\
\hline $\begin{array}{l}\text { 2016, Wang } \\
\text { and Chung } \\
{[626]}\end{array}$ & $\begin{array}{l}\text { Non-HC } \\
\text { additive }\end{array}$ & $\begin{array}{l}\text { Fuel: Ethylene } \\
\text { Topic: Effect of carbon dioxide addition on } \\
\text { PAH formation and soot evolution in ethylene } \\
\text { flames }\end{array}$ & $\begin{array}{l}\text { EXP: LE/LS, } \\
\text { LII } \\
\text { SIM: }\end{array}$ \\
\hline $\begin{array}{l}\text { 2016, Zimmer } \\
\text { et al. [627] }\end{array}$ & Soot model & $\begin{array}{l}\text { Fuel: Ethylene } \\
\text { Topic: Effects of mass and energy coupling } \\
\text { between the gas- and the solid-phase. }\end{array}$ & SIM \\
\hline $\begin{array}{l}\text { 2017, Amin } \\
\text { and Roberts } \\
\text { [529] }\end{array}$ & $\begin{array}{l}\text { Pressure } \\
\text { effects }\end{array}$ & $\begin{array}{l}\text { Fuel: Ethylene } \\
\text { Topic: Soot concentration and morphology in } \\
\text { ethylene CDFs at elevated pressures }\end{array}$ & EXP: LS/LE \\
\hline $\begin{array}{l}\text { 2017, Carbone } \\
\text { et al. [628] }\end{array}$ & $\begin{array}{l}\text { Pressure } \\
\text { effects; } \\
\text { partial } \\
\text { premixing }\end{array}$ & $\begin{array}{l}\text { Fuel: Ethylene } \\
\text { Topic: Chemical speciation of incipiently } \\
\text { sooting partially premixed flames of ethylene } \\
\text { at various pressures }\end{array}$ & $\begin{array}{l}\text { EXP: } \\
\text { GC/MS } \\
\text { SIM: }\end{array}$ \\
\hline $\begin{array}{l}\text { 2017, Hansen } \\
\text { et al. [207] }\end{array}$ & $\begin{array}{l}\text { Kinetic } \\
\text { mechanism; } \\
\text { fuel type }\end{array}$ & $\begin{array}{l}\text { Fuel: n-butane, i-butane, i-butene } \\
\text { Topic: Kinetic pathways for PAH formation } \\
\text { in flames of } \mathrm{C}_{4} \text { fuels }\end{array}$ & EXP: MBMS \\
\hline $\begin{array}{l}\text { 2017, Park et } \\
\text { al. [629] }\end{array}$ & Fuel mixing & $\begin{array}{l}\text { Fuel: n-heptane, iso-octane, toluene } \\
\text { Topic: Compositional effects on the sooting } \\
\text { tendencies of toluene primary reference fuels }\end{array}$ & $\begin{array}{l}\text { EXP: LIF, } \\
\text { LII } \\
\text { SIM }\end{array}$ \\
\hline $\begin{array}{l}2017, \\
\text { Rodrigues et } \\
\text { al. }[630]\end{array}$ & $\begin{array}{l}\text { Strain rate } \\
\text { effects }\end{array}$ & $\begin{array}{l}\text { Fuel: Propane } \\
\text { Topic: Sooting and PAH formation } \\
\text { characteristics of CDF subject to strain rate } \\
\text { harmonic oscillations }\end{array}$ & SIM \\
\hline $\begin{array}{l}\text { 2017, Xue et } \\
\text { al. [631] }\end{array}$ & Fuel type & $\begin{array}{l}\text { Fuel: Jet fuels } \\
\text { Topic: Sooting tendencies of representative } \\
\text { conventional and alternative jet fuels }\end{array}$ & EXP: LII, LE \\
\hline $\begin{array}{l}\text { 2018, Amin et } \\
\text { al. [632] }\end{array}$ & $\begin{array}{l}\text { Pressure } \\
\text { effects }\end{array}$ & $\begin{array}{l}\text { Fuel: Ethylene } \\
\text { Topic: Effects of pressure on primary particle } \\
\text { size distribution and fractal properties of soot } \\
\text { aggregates }\end{array}$ & EXP: TEM \\
\hline $\begin{array}{l}\text { 2018, Gleason } \\
\text { et al. [489] }\end{array}$ & $\begin{array}{l}\text { Temperature } \\
\text { effects; } \\
\text { sooting } \\
\text { structure }\end{array}$ & $\begin{array}{l}\text { Fuel: Ethylene } \\
\text { Topic: Effects of temperature on soot } \\
\text { evolution and optical properties in highly } \\
\text { controlled CDFs }\end{array}$ & $\begin{array}{l}\text { EXP: } \\
\text { pyrometry }\end{array}$ \\
\hline $\begin{array}{l}2018, \\
\text { Kalvakala et } \\
\text { al. }[377]\end{array}$ & $\begin{array}{l}\text { Fuel type; } \\
\text { flame structure } \\
\text { effect }\end{array}$ & $\begin{array}{l}\text { Fuel: Ethylene, propane, propene } \\
\text { Topic: Effects of fuel unsaturation and } \\
\text { stoichiometric mixture fraction on NOx, PAH } \\
\text { and soot formation }\end{array}$ & SIM \\
\hline
\end{tabular}




\begin{tabular}{|c|c|c|c|}
\hline 2018, Li, [633] & $\begin{array}{l}\text { Fuel mixing; } \\
\text { pressure } \\
\text { effects }\end{array}$ & $\begin{array}{l}\text { Fuel: Ethylene } \\
\text { Topic: Effects of DME addition to ethylene } \\
\text { on sooting limits at elevated pressures }\end{array}$ & $\begin{array}{l}\text { EXP: LS } \\
\text { SIM }\end{array}$ \\
\hline $\begin{array}{l}\text { 2018, Raj et al. } \\
\text { [634] }\end{array}$ & Fuel additive & $\begin{array}{l}\text { Fuel: Methane } \\
\text { Topic: Effects of iron pentacarbonyl addition } \\
\text { on soot formation and oxidation in methane } \\
\text { CDF }\end{array}$ & $\begin{array}{l}\text { EXP: SMPS } \\
\text { SEM }\end{array}$ \\
\hline $\begin{array}{l}\text { 2018, Sarnacki } \\
\text { and Chelliah } \\
\text { [530] }\end{array}$ & $\begin{array}{l}\text { Strain rate } \\
\text { effects; } \\
\text { pressure } \\
\text { effects }\end{array}$ & $\begin{array}{l}\text { Fuel: Ethylene } \\
\text { Topic: Effects of strain rate and pressure (up } \\
\text { to } 30 \mathrm{~atm} \text { ) on soot formation }\end{array}$ & $\begin{array}{l}\text { EXP: LII, } \\
\text { PIV }\end{array}$ \\
\hline $\begin{array}{l}\text { 2018, Wang et } \\
\text { al. [635] }\end{array}$ & Fuel type & $\begin{array}{l}\text { Fuel: Ethylene, propene, 1-butene, 1-pentene, } \\
\text { 1-hexene, 1-octene } \\
\text { Topic: Kinetic analysis on the effect of } \\
\text { carbon number atoms on soot and PAH } \\
\text { formation tendencies for 1-alkene fuels }\end{array}$ & $\begin{array}{l}\text { EXP: LII, } \\
\text { LIF } \\
\text { SIM }\end{array}$ \\
\hline $\begin{array}{l}\text { 2018, Xu et al. } \\
\text { [378] }\end{array}$ & $\begin{array}{l}\text { Dilution } \\
\text { effects; } \\
\text { flame structure } \\
\text { effects }\end{array}$ & $\begin{array}{l}\text { Fuel: Ethylene } \\
\text { Topic: Kinetic analysis on the effects of } \\
\text { oxidizer composition on soot formation in } \\
\text { both SF and SFO flames }\end{array}$ & $\begin{array}{l}\text { EXP: LE } \\
\text { SIM }\end{array}$ \\
\hline $\begin{array}{l}\text { 2018, Xue et } \\
\text { al. [636] }\end{array}$ & $\begin{array}{l}\text { Pressure } \\
\text { effects }\end{array}$ & $\begin{array}{l}\text { Fuel: Ethylene } \\
\text { Topic: Pressure effect on soot formation and } \\
\text { the implications of keeping density-weighted } \\
\text { strain rate constant }\end{array}$ & $\begin{array}{l}\text { EXP: LII } \\
\text { SIM }\end{array}$ \\
\hline $\begin{array}{l}\text { 2019, Xue et } \\
\text { al. [637] }\end{array}$ & $\begin{array}{l}\text { Fuel type; } \\
\text { fuel mixing }\end{array}$ & $\begin{array}{l}\text { Fuel: Jet-A, FT-SPK, ATJ, HEFA-camelina } \\
\text { Topic: Sooting tendencies of } \\
\text { alternative/conventional jet fuel blends }\end{array}$ & EXP: LII, LE \\
\hline $\begin{array}{l}\text { 2019, Yan et } \\
\text { al. [638] }\end{array}$ & $\begin{array}{l}\text { Soot evolution } \\
\text { process }\end{array}$ & $\begin{array}{l}\text { Fuel: Ethylene } \\
\text { Topic: Dependence of wavelength on soot } \\
\text { extinction measurements }\end{array}$ & EXP: LE \\
\hline $\begin{array}{l}\text { 2019, Gleason } \\
\text { et al. [490] }\end{array}$ & $\begin{array}{l}\text { Temperature } \\
\text { effects; } \\
\text { pressure } \\
\text { effects }\end{array}$ & $\begin{array}{l}\text { Fuel: Ethylene } \\
\text { Topic: Effects of temperature and pressure on } \\
\text { soot evolution and optical properties in highly } \\
\text { controlled CDFs }\end{array}$ & $\begin{array}{l}\text { EXP: } \\
\text { pyrometry }\end{array}$ \\
\hline $\begin{array}{l}\text { 2019, Yan et } \\
\text { al. [397] }\end{array}$ & Fuel mixing & $\begin{array}{l}\text { Fuel: Ethylene } \\
\text { Topic: Effects of methanol and ethanol } \\
\text { addition on soot formation in ethylene CDF }\end{array}$ & $\begin{array}{l}\text { EXP: LII, LE } \\
\text { SIM }\end{array}$ \\
\hline $\begin{array}{l}\text { 2019, Hoerlle } \\
\text { and Pereira } \\
{[639]}\end{array}$ & Dilution effect & $\begin{array}{l}\text { Fuel: Ethylene } \\
\text { Topic: Effects of CO2 addition in oxygen- } \\
\text { enriched CDF of ethylene }\end{array}$ & SIM \\
\hline $\begin{array}{l}2019, \\
\text { Mahmoud et } \\
\text { al. }[640]\end{array}$ & Dilution effect & $\begin{array}{l}\text { Fuel: Ethylene } \\
\text { Topic: Combined effects of } \mathrm{CO}_{2} \text { and } \mathrm{H}_{2} \mathrm{O} \\
\text { addition in fuel- and oxidizer-side of ethylene } \\
\text { CDF }\end{array}$ & $\begin{array}{l}\text { EXP: LII, LE } \\
\text { SIM }\end{array}$ \\
\hline
\end{tabular}




\subsection{Effects of fuel types on soot formation}

It is natural to imagine that certain fuels are inherently more sooting than others, and it is generally accepted that fuel molecular structure plays a vital role in determining the sooting tendency of a combustion system $[96,641]$. This point could be best viewed from the fact that flames fueled with different isomers may exhibit significantly different sooting characteristics, although they share similar flame temperature, $\mathrm{C} / \mathrm{O}$ ratio, and other flow field parameters. For instance, in counterflow flames of neat fuels and synthetic air $\left(X_{\mathrm{O}, 0}=0.25\right)$, it was observed that the soot volume fraction of iso-butane flame was three times as much as that of n-butane flame, although the two flames had nearly the same peak temperatures [113].

Fundamental understanding of the effect of fuel molecular structure on soot formation requires comprehensive knowledge of the kinetic pathways from the fuel to the molecular soot precursors, as well as the interactions between the pool of intermediate species and the soot surface growth. An important way to obtain such information is through numerical simulations with a detailed chemistry. Because of its simple quasi one-dimensional nature of flow field, the CDF configuration is particularly amenable to such detailed computation. In general, there are two types of studies that focus on fuel effects. Flames can either be established using neat fuels of the target [635] or various target fuels can be doped into a baseline flame of-for example-ethylene [642, 643] or methane [67].

Before discussing individual investigations, it is important to mention here that studies comparing the sooting tendency of different fuels should be considered in view of the changes in other parameters (e.g. stoichiometric mixture fraction and flame maximum/adiabatic temperature) that occur when using different fuels. In fact, it is always important to keep in mind that the conclusion that we draw from a specific parametric study must be understood with the effects of accompanying variations of other parameters taken into full consideration. 


\subsubsection{Counterflow diffusion flames with single component (neat) fuels}

Glassman and coworkers are among the pioneers using CDFs to study the fuel-dependence on soot formation [94, 113]. These relatively early studies utilized the configuration in which a flame is established in the forward stagnation region of a cylindrical porous burner, as schematically shown in Figure 20(a). In Ref. [113], a uniform flow of synthetic air $\left(X_{\mathrm{O}, 0}=0.25\right)$ was set to approach the porous burner with a free-stream velocity of $30 \mathrm{~cm} / \mathrm{s}$. The measurements were made along the centerline for axial velocity, temperature, and soot volume fraction/size/number density using LDV, thermocouples, and light extinction/scattering techniques, respectively. Propane, n-butane, and isobutane fuels were tested, and the soot-related results are summarized in Figure 20 (b). Note that in the original work [113], additional flames of alkene fuels such as ethylene and propene were also established; however, the oxidizer concentrations $\left(X_{\mathrm{O}, 0}\right)$ used were different than those used for alkane fuels, making a direct comparison of sooting tendencies across these different fuel classes difficult. Therefore, only the results for alkane fuels are re-plotted in Figure 20. 
(a)
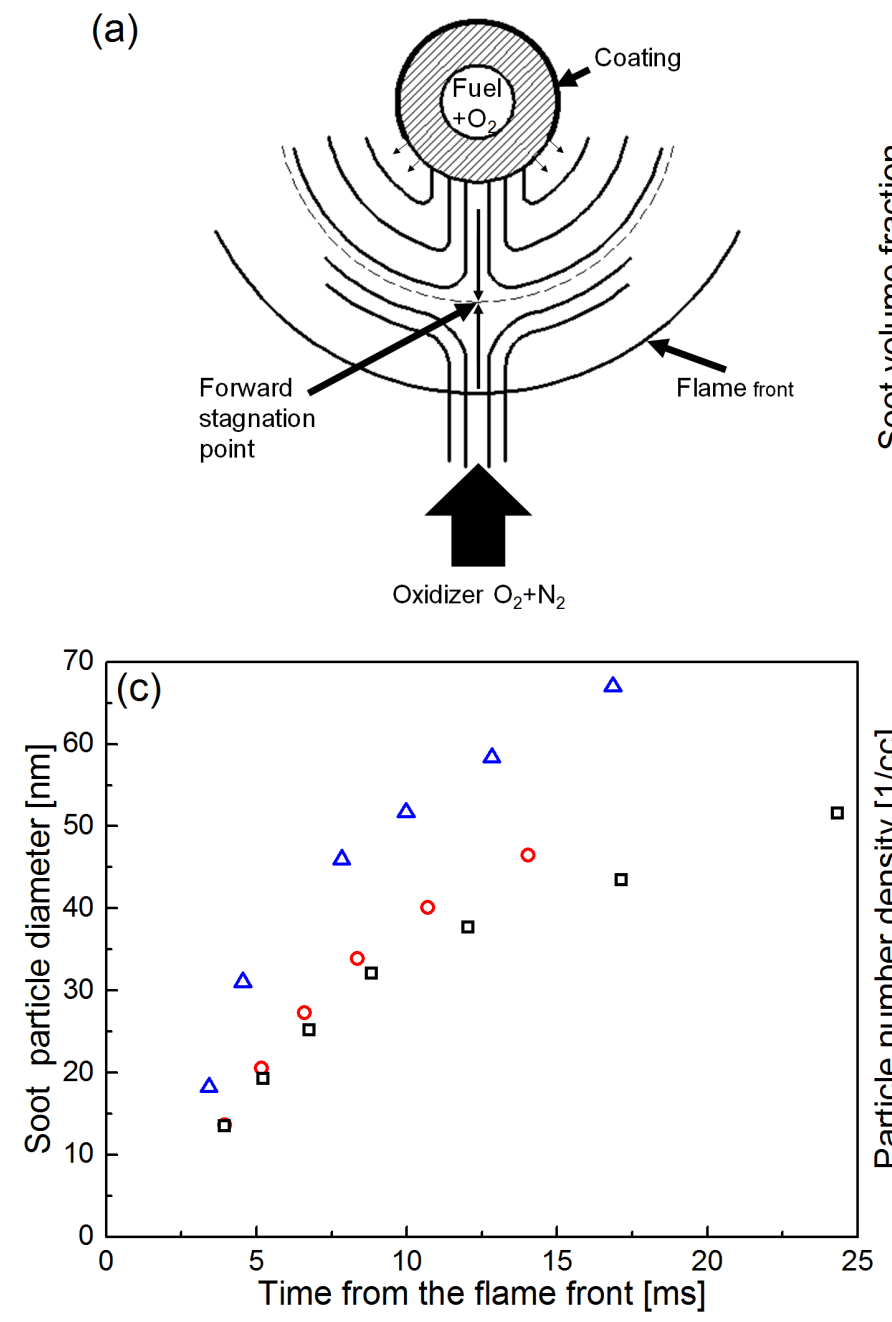
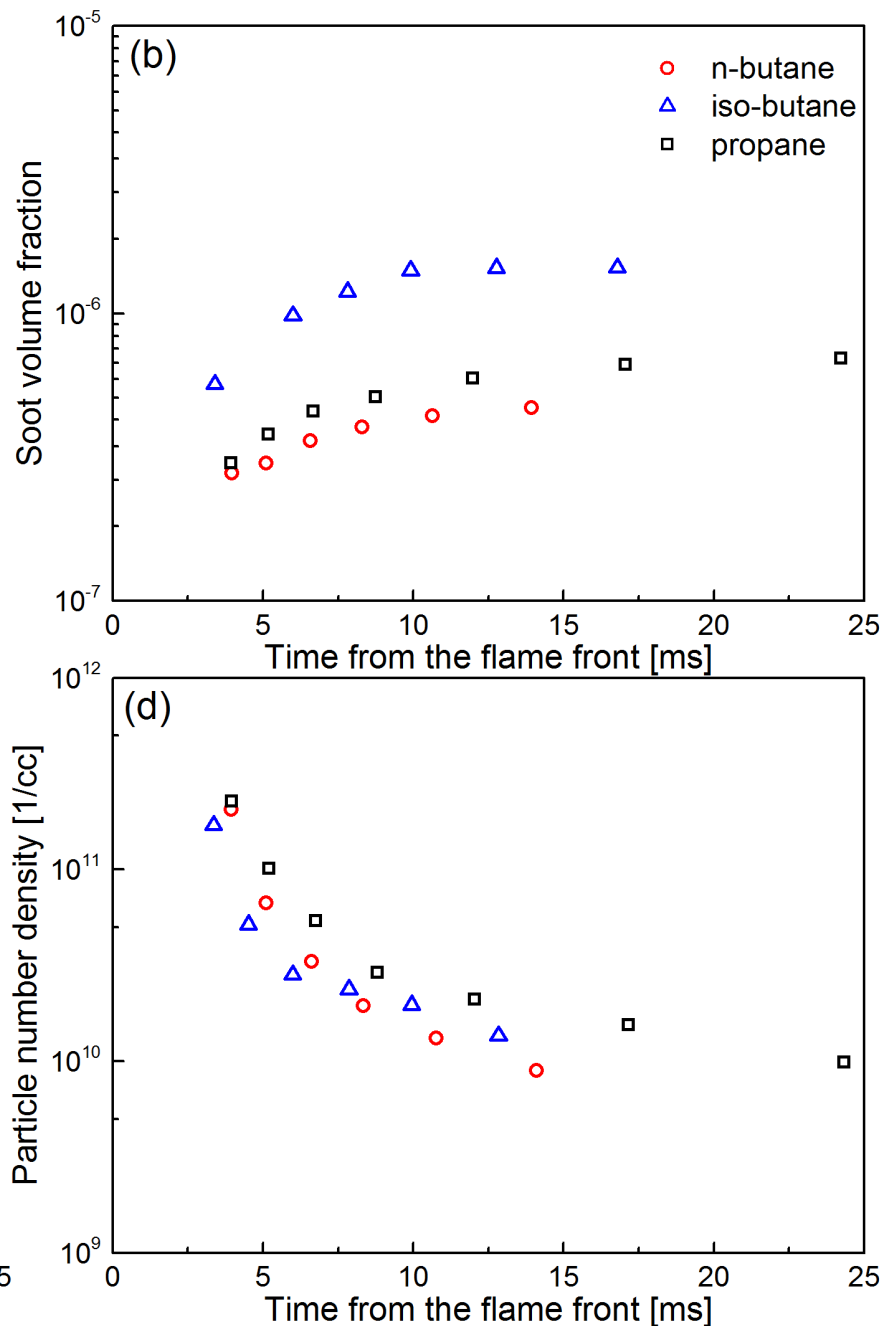

Figure 20. Schematic of counterflow flow diffusion flame stabilized around a porous cylindrical burner (a), redrawn from Hura and Glassman [113]; Measured soot volume fraction (b), average particle size (c), and number density (d) as a function of time from the flame front towards the burner, data taken from Hura and Glassman [113].

As can be seen, the iso-butane flame generated the highest level of soot, followed by propane and n-butane (b). In addition, the higher sooting fuels also produced larger soot particles (c). However, the number density profiles (d) were confined in a relatively narrow band and exhibited less variation among the different fuels, believed to be consistent with the behavior of a coagulation-dominated aerosol system. Due to the lack of well-validated soot mechanisms that were able to handle multiple fuels in the early years, the authors did not attempt to explain these experimentally-observed trends through direct soot simulations of the CDF. Instead, inferences were taken from relevant iso-thermal 
pyrolysis studies and it was suggested that the sooting tendency was dependent not only on fuel pyrolysis rates but also on the intermediate species formed during the pyrolysis process [96, 113, 644]. This observation, regarding iso-butane's higher sooting tendency as compared to n-butane, was consistent with an earlier ranking using the smoke point data [645]. It was also corroborated by a number of subsequent studies [86, 231, 620]. In particular, it was pointed out that the decomposition of iso-butane leads to rather high levels of propene and iso-butene [646], which can be more readily converted to propargyl, and subsequently to benzene [152], as compared to $C_{2}$ species, which are among the major decomposition products of n-butane [647]. An exception comes from the work of Singh et al. [84], where it was observed that the peak soot volume fraction of the n-butane CDF was notably higher than that of iso-butane flame under the same conditions of $X_{\mathrm{F}, 0}=0.29, X_{\mathrm{O}, 0}=0.9$, and effective strain rate $K_{\mathrm{E}}=57 \mathrm{~s}^{-1}$. As an attempt to rationalize their experimental results, the authors performed kinetic simulations of the respective flames using both the Merchant [648] and the USC II mechanisms [649]. The numerical results from the two mechanisms consistently showed that although n-butane flame had a higher concentration of $\mathrm{C}_{2} \mathrm{H}_{2}$, it featured a notably lower level of $\mathrm{C}_{3} \mathrm{H}_{3}$ than iso-butane flames. This is consistent with our previous discussions on the decomposition products of the two butane isomers. Further considering the importance of the propargyl recombination reaction in forming aromatic species, it was expected (and later confirmed by PAH LIF experiments [441] ) that iso-butane would produce more aromatic soot precursors. These findings allowed the authors to conclude that the ranking of soot tendencies may not be consistent with concentrations of aromatics in the inception stage [84, 441]. However, further research is still needed to clarify the details of how the trends became reversed during soot growth processes.

Using an opposed-nozzle counterflow configuration, Wang and Chung [620] performed experimental work on the sooting limit to rank the sooting tendencies of various $\mathrm{C}_{1}-\mathrm{C}_{4}$ gaseous hydrocarbons. The results showed that for SF flames, propane and n-butane have comparable sooting tendencies, which are notably higher than those of methane and ethane. In addition, the soot formation 
with $C_{2}$ and $C_{3}$ alkene fuels was significantly higher than ethane and propane, respectively, and the branched iso-butane was more sooting than its normal-chain counterparts. Kalvakala et al. [377] computationally investigated the effects of fuel unsaturation on soot formation by comparing the predicted peak soot volume fraction among counterflow diffusion flames of ethylene, propane, and propene. In agreement with the experimental results of Wang and Chung [620], they observed that the computed sooting tendency follows propene $>$ ethylene $>$ propane, regardless of the stoichiometric mixture fractions at which the comparisons were made [377]. Accompanying kinetic simulations conducted at $X_{\mathrm{F}, 0}=1.0$ and $X_{\mathrm{O}, 0}=0.21$ demonstrated that the major benzene formation pathway was similar in both propane and propene flames, being the recombination of propargyl radicals. As such, their different sooting tendency was largely a result of the different capability of the respective fuel pyrolysis products in forming propargyl radicals. Due to the presence of the double bond, the $\mathrm{C}-\mathrm{H}$ bond of the $\gamma$ carbon had the lowest bond energy, so that a major decomposition product of propene is allyl radical $\left(\mathrm{a}-\mathrm{C}_{3} \mathrm{H}_{5}\right)$, which through multiple hydrogen abstractions can be readily converted to produce $\mathrm{C}_{3} \mathrm{H}_{3}$. However, for propane $\left(\mathrm{C}_{3} \mathrm{H}_{8}\right)$ molecules, the $\mathrm{C}-\mathrm{C}$ bond was relatively weaker than the $\mathrm{C}-\mathrm{H}$ bond, so that ethyl radical became the most dominant decomposition product, while $\mathrm{C}_{3}$ species like n-propyl and propene became secondary. The fact that propane was less sooting than propene was then rationalized by noting that it is significantly more difficult to form $\mathrm{C}_{3} \mathrm{H}_{3}$ from the main decomposition products of $\mathrm{C}_{3} \mathrm{H}_{8}$ (i.e., $\mathrm{C}_{2}$ species) than from unsaturated $\mathrm{C}_{3}$ species.

The effects of unsaturation on $\mathrm{PAH} /$ soot formation were also investigated for $\mathrm{C}_{7}$ hydrocarbon fuels (n-heptane and 1-heptene) in CDFs $[610,611]$. Utilizing a detailed reaction mechanism and a PAHbased soot model, Fu et al. [610] showed that 1-heptene flame has a consistently higher soot number density, average particle size, and soot volume fraction as compared to the n-heptane flame, although both flames had very similar heat release characteristics. Furthermore, the numerical results also indicated that the existence of the double bond exerted increasingly pronounced effects on PAH and soot formation as the flame strain rates and/or levels of fuel stream partial premixing increased. 
Alkene fuels are important constituents of practical hydrocarbon fuels [650,651]; they are also key intermediates during alkane oxidation and pyrolysis [652]. As such, detailed knowledge of alkene combustion is required if the combustion and pollutant formation mechanisms of more practical fuels are to be understood. Motivated by this, Wang et al. [635] performed a systematic investigation on the sooting tendencies of $\mathrm{C}_{2}-\mathrm{C}_{8} 1$-alkene fuels in CDFs. The experimental results from the LII and PAH LIF measurements, summarized in Figure 21, indicated that 1-butene (ethylene) flame produced most (least) soot and PAHs, and the sooting tendency decreased with the increase in the number of carbon atoms for fuels heavier than $1-\mathrm{C}_{4} \mathrm{H}_{8}$. Accompanying numerical simulation confirmed and provided kinetic insights into these experimental trends.

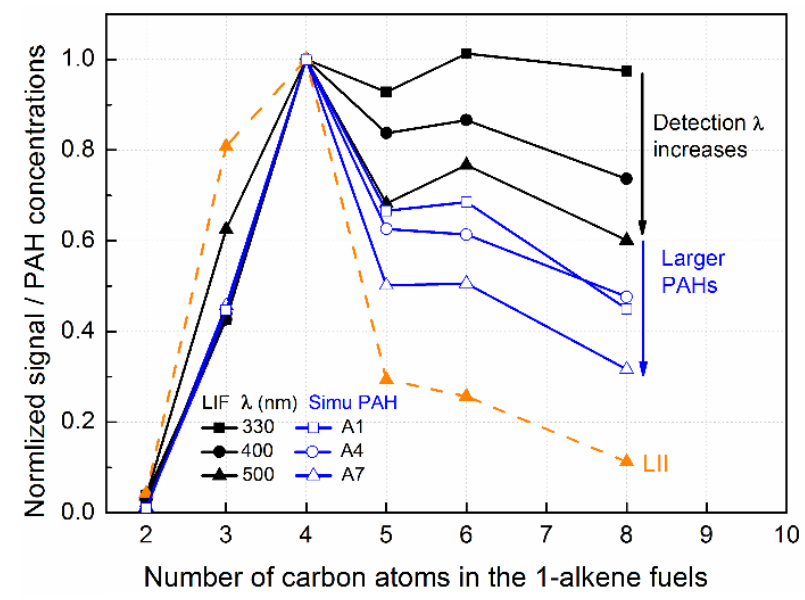

Figure 21. Experimental peak PAH LIF signals detected at various wavelengths and peak soot LII signals in counterflow diffusion flames of 1-alkane fuels with 2-8 carbon atoms. Computed peak PAH concentrations were included. All data were normalized by the values for 1-butene flame. Reprinted from Wang et al. [635] with permission of Elsevier.

In addition to the above studies focusing on conventional hydrocarbon fuels, there are also CDFbased investigations on $\mathrm{PAH} /$ soot formation of oxygenated/bio-derived fuels. For instance, using a GC system with micro-probe sampling, Frassoldati et al. [601] quantified the mole fractions of stable intermediate species in both n-propanol and iso-propanol CDFs with $Y_{\mathrm{F}, 0}=0.3, Y_{\mathrm{O}, 0}=0.233$, and $K_{\mathrm{E}}$ $=97.5 \mathrm{~s}^{-1}$. They found that the iso-propanol flame had significantly higher level of $\mathrm{C}_{4} \mathrm{H}_{6}$ and $\mathrm{C}_{3} \mathrm{H}_{6}$, both of which have a pronounced tendency to form aromatic species-and thus-soot $[174,635]$. Singh 
et al. compared the soot [84] and PAH [441] formation tendencies of four butanol isomers in CDF with $X_{\mathrm{F}, 0}=0.29$ and $X_{\mathrm{O}, 0}=0.90$ (diluted by $\mathrm{N}_{2}$ ) and observed that tert-butanol was the most sooting fuel, followed in decreasing order by n-butanol, iso-butanol, and sec-butanol. Although their results regarding the relative sooting tendency between n-butanol and iso-butanol (i.e., n-butanol is more sooting than iso-butanol) differed from previous studies in premixed flames [231] and yield soot index (YSI) rankings measured in coflow diffusion flames [653], the authors did observe that isobutanol had a higher tendency than n-butanol to form small-sized PAHs, consistent with quantitative benzene measurements in coflow diffusion flames [646, 654]. Interestingly, LIF signals with longer detection wavelength ( $>450 \mathrm{~nm}$, typically believed to represent larger PAHs of more than four rings) showed that n-butanol has a greater tendency to form larger PAHs [441], in agreement with the ranking for sooting tendency. While no definite explanations were provided of this interesting trend reversal, it must be pointed out that in Ref. [441], due to large $X_{\mathrm{O}, 0}$ of 0.9 , the flames from Singh and coworkers featured a rather different stoichiometric mixture fraction, and thus different flame structures, than the coflow fuel-air flames. It is likely that the ranking of sooting tendencies are dependent upon flame structures.

Sarathy and coworkers [589, 655] compared the chemical structures of methyl-butanoate $\left(\mathrm{CH}_{3} \mathrm{CH}_{2} \mathrm{CH}_{2} \mathrm{C}(=\mathrm{O}) \mathrm{OCH}_{3}\right)$ and methyl-crotonate $(\mathrm{CH} 3 \mathrm{CH}=\mathrm{CHC}(=\mathrm{O}) \mathrm{OCH} 3)$ diffusion flames in a counterflow configuration. The experimental results showed that the methyl-crotonate flame consistently produced higher levels of unsaturated species, such as $\mathrm{C}_{2} \mathrm{H}_{2}, 1-\mathrm{C}_{3} \mathrm{H}_{4}, 1-\mathrm{C}_{4} \mathrm{H}_{8}, 1,3-\mathrm{C}_{4} \mathrm{H}_{6}$, as well as $\mathrm{C}_{6} \mathrm{H}_{6}$, and is thus believed to have a higher propensity to soot than the methyl-butanoate flame. In a step further, Feng et al. [82] systematically measured the soot concentrations in CDFs for a class of model biodiesel fuels, emphasizing the effects of the type of ester functional group, number of carbon atoms, and double bonds. Since some of the fuels tested were of high molecular weight (up to methyl-undecanoate), and thus with rather low vapor pressure, these fuels were highly diluted with $\mathrm{N}_{2}\left(X_{\mathrm{F}, 0} \sim 0.1\right)$ to maintain a moderate partial pressure. To promote soot formation for sensitive 
measurements, pure oxygen was used as the oxidizer stream, which rendered the flames with SFO characteristics [73]. The study, summarized in Figure 22, showed that for the same functional ester group, the maximum soot volume fractions in the CDFs increased with the increase in fuel carbon number. Also, for esters with the same number of carbon atoms, methyl esters exhibited less sooting tendency than ethyl esters, and the unsaturated esters were significantly more sooting than their saturated counterparts. Nevertheless, the location of the double bond did not notably affect sooting tendencies.
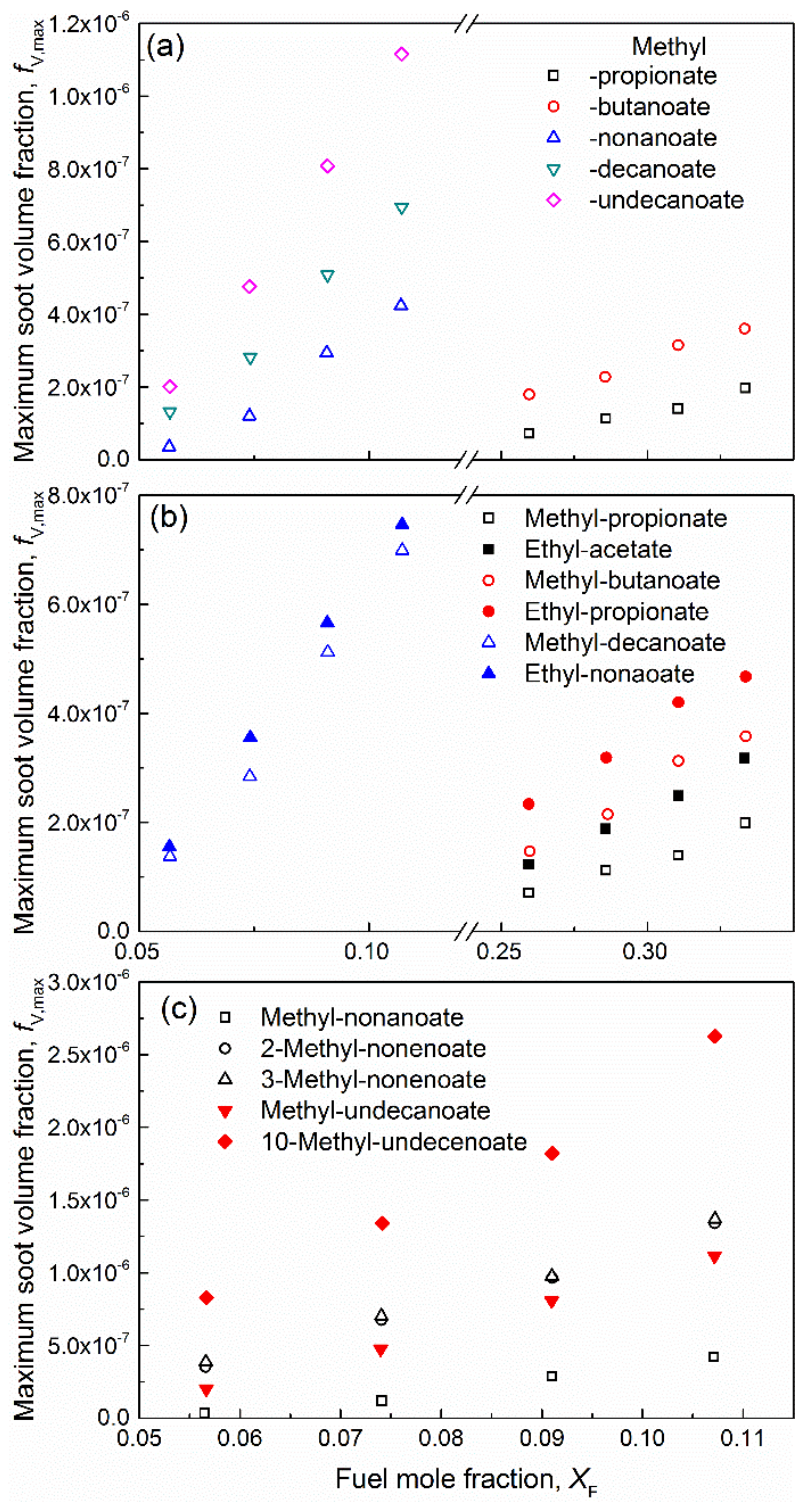

Figure 22. Peak measured soot volume fractions of CDFs of various ester fuels: effects of length of carbon chain (a); comparison between methyl- and ethyl- esters (b); and effects of fuel unsaturation (c), adapted from Feng et al. [82] with permission of Elsevier. 


\subsubsection{Counterflow diffusion flames with fuel mixtures}

It is noted that there are even more studies utilizing fuel mixtures than single-component fuels in CDF-based soot investigations on fuel effects. This popularity of the study of fuel mixing effects may be attributed to the following: 1) Conventional petroleum-based fuels and natural gases are always complex fuel mixtures [656], making understanding compositional effects on soot formation of practical importance; 2) The chemical interactions of various fuel molecules can sometimes shed light on important kinetic pathways for soot formation that are elusive in studies of single component fuels (a notable example is the synergistic effects of ethylene and propane mixtures on soot formation [528], detailed later); 3) The sooting tendencies of some fuels (i.e., high MW aromatic fuels) may be too high for accurate experimental determination of their sooting characteristics, and to study the relative effects in such cases it is usually beneficial to mix a small amount of the target fuel to a baseline flame.

\section{a) Effects of $\mathrm{CO} / \mathrm{H}_{2}$ addition}

Du et al. [568] investigated the effects of $\mathrm{CO}$ and $\mathrm{H}_{2}$ addition in the fuel stream on soot inception rates in $\mathrm{CDFs}$ of $\mathrm{C}_{2} \mathrm{H}_{4}, \mathrm{C}_{3} \mathrm{H}_{8}$ and $\mathrm{n}-\mathrm{C}_{4} \mathrm{H}_{10}$ fuels. Because the additives can passively affect soot formation by altering fuel concentration and flame temperature (in addition to directly influencing kinetic reactions), the authors conducted additional contrast tests by adding inert species with physical properties comparable to the target dopant. Of special note is that helium was used to contrast hydrogen for the isolation of the chemical effects of the latter. This choice was justified, since helium has a high diffusivity, similar to $\mathrm{H}_{2}$, while it was shown that the preferential diffusion effects of these highly mobile species play important roles in strained flames [657-659]. The tendency for soot formation in the CDFs was quantified by the critical (minimum) local strain rate ( $K_{\mathrm{cr}}$, as measured by laser doppler velocimetry), at which light scattering signals from soot particles disappear (i.e., soot 
inception is inhibited $[551,660])$. Therefore, a higher $K_{\text {cr }}$ implies a stronger sooting tendency. The experimental results, summarized in Figure 23, showed that the addition of CO inhibited soot formation in $\mathrm{C}_{2} \mathrm{H}_{4}$ flames, while its addition in $\mathrm{C}_{3} \mathrm{H}_{8}$ and $\mathrm{n}-\mathrm{C}_{4} \mathrm{H}_{10}$ flames had an interesting nonmonotonic effect. Further analysis showed that $\mathrm{CO}$ exerted its soot-inhibitive effects in $\mathrm{C}_{2} \mathrm{H}_{4}$ flames mainly through fuel dilution. On the other hand, the initial enhancement of the sooting tendency with $\mathrm{CO}$ addition in $\mathrm{C}_{3} \mathrm{H}_{8}$ and $n-\mathrm{C}_{4} \mathrm{H}_{10}$ flames was thought to be caused by its chemical effects through reaction $\mathrm{CO}+\mathrm{OH}=\mathrm{CO}_{2}+\mathrm{H}$, which may alter the concentrations of $\mathrm{H}$ and $\mathrm{OH}$ radicals that are important for soot formation and oxidation, respectively. Nevertheless, the authors did not provide direct evidence of this chemical effect and acknowledged that such reasoning could not explain the inhibiting behavior of $\mathrm{CO}$ in $\mathrm{C}_{2} \mathrm{H}_{4}$ flames [568]. Interestingly, in a subsequent study of coflow diffusion ethylene flames by Guo et al.[387], a detailed kinetic model suggested that in addition to the dilution effect, $\mathrm{CO}$ plays a chemically-enhancing effect on soot formation by: 1) raising flame temperature; 2) increasing the surface growth rates due to higher $\mathrm{H}$ radical production; and 3) decreasing soot oxidation due to higher $\mathrm{OH}$ radical consumption. They noticed that the overall soot production rate was reduced, implying that the dilution effect was still dominant. However, no further study was conducted to explain the experimental results of Du et al. [568] that soot inception was enhanced by $\mathrm{CO}$ addition in $\mathrm{C}_{3} \mathrm{H}_{8}$ and $n-\mathrm{C}_{4} \mathrm{H}_{10}$ CDF flames. $\mathrm{CO}$ may have a role in influencing fuel pyrolysis chemistry, but further studies are needed. 

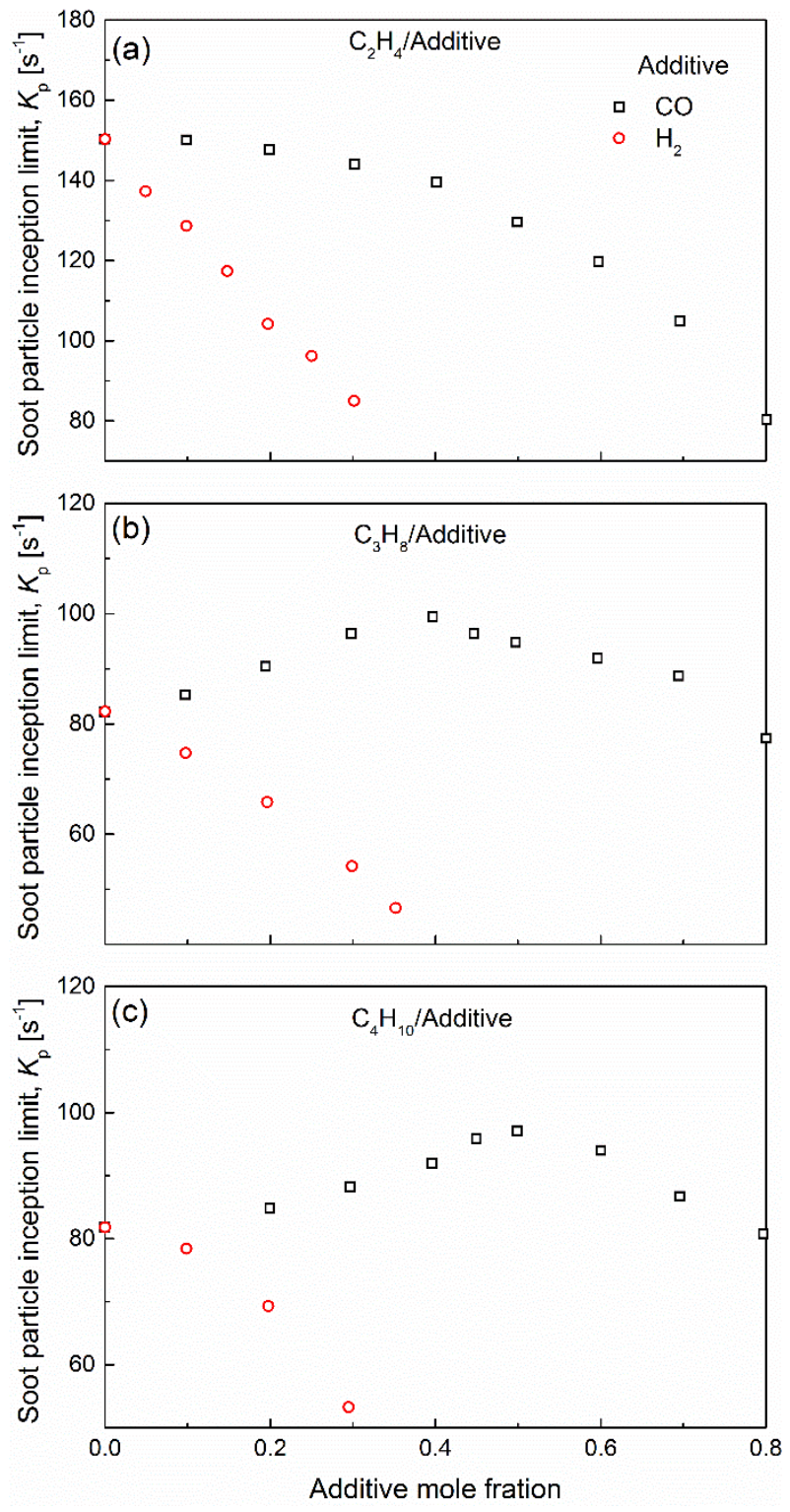

Figure 23. Effects of $\mathrm{CO} / \mathrm{H}_{2}$ addition in the fuel side on critical strain rates for soot formation in CDFs with base fuels of $\mathrm{C}_{2} \mathrm{H}_{4}(\mathrm{a}) ; \mathrm{C}_{3} \mathrm{H}_{8}$ (b) and n- $\mathrm{C}_{4} \mathrm{H}_{10}$ (c). Adapted from Du et al. [568] with permission of Elsevier.

The addition of $\mathrm{H}_{2}$ was found to decrease the sooting tendencies of CDFs of $\mathrm{C}_{2} \mathrm{H}_{4}, \mathrm{C}_{3} \mathrm{H}_{8}$ and n$\mathrm{C}_{4} \mathrm{H}_{10}$ fuels [568], even though flame temperatures were always enhanced. The following were believed to offset soot-promoting effects of higher flame temperature from $\mathrm{H}_{2}$ addition: 1) In positively stretched flames, the preferential diffusion effects of $\mathrm{H}_{2}$ may cause precursor concentration reduction, decreasing the rate of soot formation; 2) In the HACA framework for PAH growth [661], the addition of $\mathrm{H}_{2}$ may push the hydrogen abstraction reaction $\mathrm{A}_{\mathrm{i}}+\mathrm{H}=\mathrm{A}_{\mathrm{i}^{-}}+\mathrm{H}_{2}$ backward, preventing 
aromatic radicals $\left(\mathrm{A}_{\mathrm{i}^{-}}\right)$from participating in further mass addition reactions, and leading finally to reduced soot precursor concentrations; 3) The addition of $\mathrm{H}_{2}$ also decreases the fuel carbon supply and thus effectively plays a role of dilution. As previously mentioned, comparison against helium addition cases may help isolate the above effects. However, it has been noted that the relative effectiveness of $\mathrm{H}_{2}$ and helium in inhibiting soot formation is not always consistent. For example, in the CDFs of Du et al. [568], helium was more effective than hydrogen in suppressing soot inception for all the three fuels tested, consistent with a study on methane coflow flames [662]. But studies in ethylene/air coflow flames $[663,664]$ showed exactly the opposite, i.e., hydrogen was more effective than helium in reducing soot formation. Compared to helium, the addition of hydrogen increases flame temperature while it may also inhibit PAH/soot HACA growth. Thus, it is highly likely that the dominating effect is dependent upon individual flame conditions. Obviously, more studies are needed to clarify these interesting phenomena.

Through visual observation of soot luminosity, Choi et al. [624] also noticed a reduction of soot formation by $\mathrm{H}_{2}$ addition in ethylene-air CDFs. Thermophoretic sampling of the soot particles and subsequent analysis with HRTEM showed that the size of the particles was reduced, while the formation of fullerenic nanostructures (represented by the curved carbon lamella) was enhanced by the addition of hydrogen. However, the exact location where soot extraction was performed in the flames was not reported. The authors postulated that the enhanced curved structure may have been a result of enhanced five-member ring formation after hydrogen addition, although no further evidence was shown.

\section{b) Synergistic effects of ethylene-based binary fuel mixtures}

The influence of fuel-stream mixing of ethylene and propane on soot formation was investigated by Hwang et al. [528] in CDFs with $X_{\mathrm{F}, 0}=1.0, X_{\mathrm{O}, 0}=0.24$ and $K_{\mathrm{E}}=55 \mathrm{~s}^{-1}$ at various fuel mixing ratios. Soot volume fractions and relative PAH concentrations were measured with light extinction/scattering and laser-induced fluorescence, respectively. An interesting synergistic effect 
was observed, i.e., PAH/soot formation was enhanced in the flames of ethylene/propane mixtures compared to both neat ethylene and neat propane flames. Specifically, the PAH/soot concentrations of the ethylene-propane flames were observed to increase first and then decrease with more propane mixing, as can be seen in Figure 24. It was argued that the monotonic changes in flame temperature and $\mathrm{C}_{2} \mathrm{H}_{2}$ concentrations with the propane mixing ratio cannot explain such non-monotonic variation in PAH/soot formation, so that chemical cross-linking between the fuel molecules must play a role. In this regard, kinetic modelling of the flames was conducted and the results showed that the peak concentration of $\mathrm{C}_{3} \mathrm{H}_{3}$ (known to be an important benzene precursor [152]) also exhibited a nonmonotonic variation with the propane mixing ratio. Further kinetic analysis $[528,616]$ revealed that the relatively low concentration of $\mathrm{C}_{3} \mathrm{H}_{3}$ in the sooting region of the neat ethylene flame acted as a bottleneck to the formation of aromatic soot precursors, while with a small amount of propane addition, the concentrations of methyl radicals $\left(\mathrm{CH}_{3}\right)$ increased as a direct consequence of propane decomposition. The increased $\mathrm{CH}_{3}$ concentrations then promoted various reactions between $\mathrm{C}_{1}$ and $\mathrm{C}_{2}$ species, leading to an enhancement of $\mathrm{C}_{3} \mathrm{H}_{3}$ [642], and finally-benzene production. As an important bridge toward large PAHs and soot particles, the increase in benzene concentration translated to the observed increase in $\mathrm{PAH} /$ soot when a small amount of propane was added to the ethylene baseline flame. With further increases in the propane mixing ratio, the concentration of $\mathrm{C}_{2} \mathrm{H}_{2}$ decreased notably, contributing negatively to PAH/soot growth through the HACA mechanism and leading to a decrease in sooting tendency. In this way, the study by Hwang et al. [528] demonstrates the interplay between $\mathrm{C}_{3} \mathrm{H}_{3}$ and $\mathrm{C}_{2} \mathrm{H}_{2}$ in determining the final soot loading of ethylene-propane CDFs, with $\mathrm{C}_{3} \mathrm{H}_{3}$ controlling incipient aromatic ring formation and $\mathrm{C}_{2} \mathrm{H}_{2}$ dominating large $\mathrm{PAH}$ and soot mass growth. In a subsequent effort to identify the role of $\mathrm{C}_{3}$ chemistry in PAH growth beyond benzene, Lee et al. [582] measured PAH and soot concentrations in CDFs of ethylene-propane mixtures with a small amount of benzene addition (1.83\%, molar concentration) to the fuel stream. It was argued that benzene doping would significantly increase benzene concentration in the pyrolysis 
and flame zone, thereby mitigating the relative importance of the effect of $\mathrm{C}_{3}$ pathways on initial ring formation to the ethylene/propane synergistic behaviors. LIF signals, detected at different wavelengths, showed that although the synergistic effect nearly disappeared in small aromatic species (represented by LIF signal with $330 \mathrm{~nm}$ detection wavelength), it was still pronounced in relatively larger PAHs (>400 $\mathrm{nm}$ detection wavelength). These results suggested that adding propane to ethylene affects not only the incipient ring formation but also PAH growth beyond benzene.

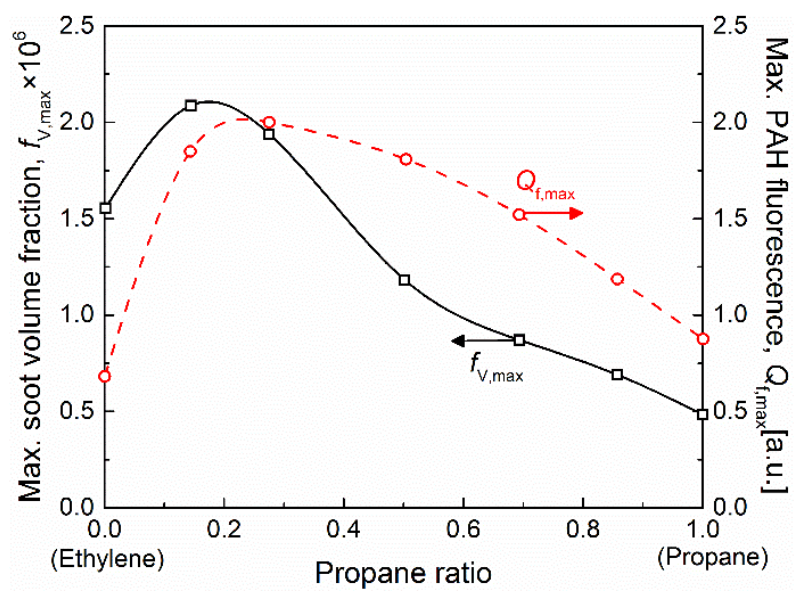

Figure 24. Synergistic effect on PAH and soot in ethylene-propane flames. Shown are peak soot volume fraction and PAH LIF signals as a function of propane mixing ratio. A non-monotonic variation can be clearly identified. Reprinted from Hwang et al. [528] with permission of Elsevier.

In addition to the influence on benzene formation through $\mathrm{C}_{3} \mathrm{H}_{3}$ recombination, Trottier et al. [665] argued (based on combined experimental and numerical investigations on soot formation in coflow diffusion flames of binary fuel mixtures) that the increase in $\mathrm{CH}_{3}$ radical from propane in ethylenepropane flame may enhance the forward reaction of $\mathrm{C}_{2} \mathrm{H}_{4}+\mathrm{CH}_{3}=\mathrm{C}_{2} \mathrm{H}_{3}+\mathrm{CH}_{4}$, increasing the concentration of vinyl radicals $\left(\mathrm{C}_{2} \mathrm{H}_{3}\right)$, which in turn contribute to higher benzene production through various $\mathrm{C}_{4}+\mathrm{C}_{2}$ channels. This argument was supported by numerical analyses of Liu et al. [284], McNesby et al. [101], and Park et al. [666]. 
It is important to note that for both the $\mathrm{C}_{3}+\mathrm{C}_{3}$ or $\mathrm{C}_{2}+\mathrm{C}_{4}$ benzene formation pathways, the $\mathrm{CH}_{3}$ radical, a direct pyrolysis product from propane decomposition, always plays a critical role in explaining the synergistic effect seen in ethylene-propane mixtures. It can thus be reasoned that the sooting tendency of ethylene flame may also be enhanced by other fuel dopants, as long as such dopant can decompose to produce appreciable amounts of $\mathrm{CH}_{3}$ radicals in the fuel pyrolysis zone. If-as a neat fuel-the dopant also has a lower sooting tendency than ethylene, synergistic effects are then expected to develop between ethylene and this dopant. Besides $\mathrm{C}_{3} \mathrm{H}_{8}$, many other fuels, including $\mathrm{CH}_{4}, \mathrm{C}_{2} \mathrm{H}_{6}$, n-butane, iso-butane, dimethyl ether $\left(\mathrm{CH}_{3}-\mathrm{O}-\mathrm{CH}_{3}, \mathrm{DME}\right)$, ethanol $\left(\mathrm{C}_{2} \mathrm{H}_{5}-\mathrm{OH}\right)$ and npropanol are known to meet these criteria. And indeed, the synergistic effects on PAH/soot formation have been observed for $\mathrm{C}_{2} \mathrm{H}_{4} / \mathrm{C}_{2} \mathrm{H}_{6}[586], \mathrm{C}_{2} \mathrm{H}_{4} / \mathrm{n}-\mathrm{C}_{4} \mathrm{H}_{10}$ [86], $\mathrm{C}_{2} \mathrm{H}_{4} / \mathrm{i}-\mathrm{C}_{4} \mathrm{H}_{10}$ [86], $\mathrm{C}_{2} \mathrm{H}_{4} / \mathrm{DME}$ [90, 100, 476], $\mathrm{C}_{2} \mathrm{H}_{4} / \mathrm{C}_{2} \mathrm{H}_{5} \mathrm{OH}[41,101,607,667], \mathrm{C}_{2} \mathrm{H}_{4} / \mathrm{C}_{3} \mathrm{H}_{7} \mathrm{OH}$ [666] mixtures, as summarized in Figure 25. The peak soot volume fractions in CDFs of these ethylene-based binary mixture fuels were plotted as a function of molar mixing ratio, after being normalized by their respective values in the neat ethylene flames. Although not all the above studies were accompanied by detailed kinetic investigations, the analyses of reaction pathways for those with unanimously pointed to the role of $\mathrm{CH}_{3}$ in inducing synergistic effects (through $\mathrm{C}_{1}$ and $\mathrm{C}_{2}$ interactions, as discussed previously). The strength of the synergistic effects (i.e., the quantitative extent by which the dopant can increase PAH/soot formation as compared to the ethylene baseline flame) were seen to be largely dependent on the relative ease for the dopant to decompose to $\mathrm{CH}_{3}$ radicals in the flame pyrolysis zone. For instance, the fact that $5 \%$ of propane addition in ethylene CDF increased the peak SVF by $32 \%$ (larger than the $14 \%$ as induced by $5 \%$ ethane addition) can be partially explained by the higher $\mathrm{CH}_{3}$ concentration in the case of propane doping [140]. The stronger synergistic effect on benzene and soot formation of ethylene/DME mixture than in ethylene/ethanol mixture flames can also be rationalized by the higher production rates of $\mathrm{CH}_{3}$ in $\mathrm{DME}$ pyrolysis than ethanol [642]. 


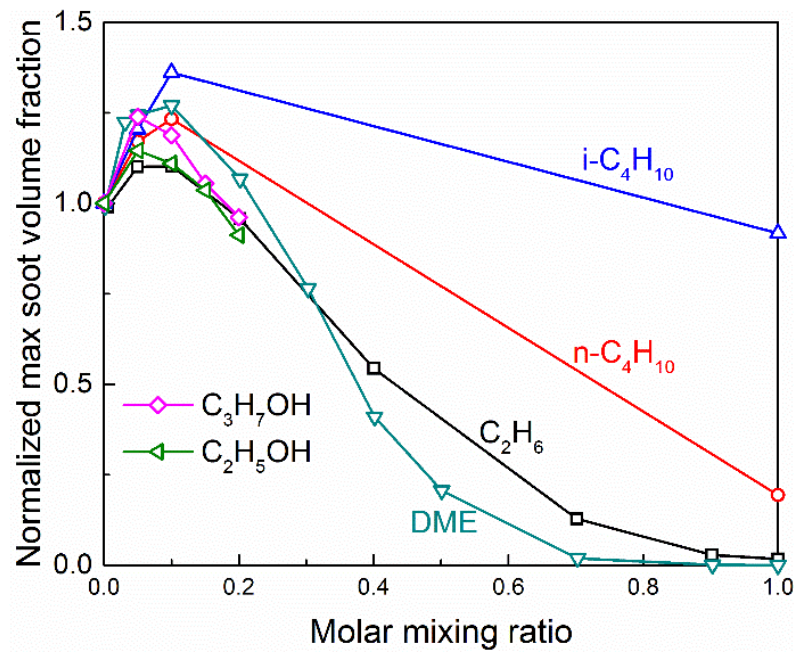

Figure 25. Normalized peak soot volume fraction in counterflow diffusion flames of ethylene-based binary mixture fuels. Data for $\mathrm{n}-\mathrm{C}_{4} \mathrm{H}_{10}$ and $\mathrm{i}-\mathrm{C}_{4} \mathrm{H}_{10}$ flames from Karatas et al. [86]. Data for $\mathrm{C}_{2} \mathrm{H}_{6}$ and DME flames from Yoon et al. [100, 586]; alcohol addition data from Park et al. [666].

The effect of $\mathrm{CH}_{4}$ addition in $\mathrm{C}_{2} \mathrm{H}_{4} \mathrm{CDF}$ deserves special attention. The sooting tendency of $\mathrm{CH}_{4}$ is known to be the lowest among all hydrocarbon fuels, which may be rationalized by the fact that $\mathrm{CH}_{4}$ has the highest $\mathrm{H} / \mathrm{C}$ ratio and there is no $\mathrm{C}-\mathrm{C}$ bond in the $\mathrm{CH}_{4}$ molecule. Nevertheless, considering that $\mathrm{CH}_{3}$ can be formed by hydrogen abstraction from $\mathrm{CH}_{4}$, it is expected that a small amount of $\mathrm{CH}_{4}$ addition in $\mathrm{C}_{2} \mathrm{H}_{4}$ flame would enhance soot formation. However, a relatively monotonic decrease in soot volume fraction with $\mathrm{CH}_{4}$ mixing ratio was observed experimentally in ethylene CDFs [586]. On the contrary, both experiments [586] and numerical simulation [140] suggested increased $\mathrm{PAH}$ formation by $\mathrm{CH}_{4}$ addition, although its quantitative extent was rather small compared to cases with other dopants (e.g., $\mathrm{C}_{2} \mathrm{H}_{6}$ and $\mathrm{C}_{3} \mathrm{H}_{8}$ ). Yoon et al. [586] explained this interesting behavior of $\mathrm{CH}_{4}$ doping based on the following: First, the $\mathrm{C}-\mathrm{H}$ bond energy $(483.6 \mathrm{~kJ} / \mathrm{mol})$ in $\mathrm{CH}_{4}$ is notably higher than the $\mathrm{C}-\mathrm{C}$ bond energy in $\mathrm{C}_{2} \mathrm{H}_{6}(376.1 \mathrm{~kJ} / \mathrm{mol})$ and $\mathrm{C}_{3} \mathrm{H}_{8}(371.3 \mathrm{~kJ} / \mathrm{mol})$, so it is more difficult for $\mathrm{CH}_{4}$ to break into $\mathrm{CH}_{3}$ radicals than for $\mathrm{C}_{2} \mathrm{H}_{6}$ and $\mathrm{C}_{3} \mathrm{H}_{8}$, especially in the low temperature region (1000-1500K, fuel side of the flame) where PAHs were formed. Therefore, the concentration of $\mathrm{CH}_{3}$ in the methane mixing case was rather low, resulting in the weaker synergistic effect on PAH formation compared to other cases. Second, the flame with $\mathrm{CH}_{4}$ mixing 
has lower flame temperature and $\mathrm{C}_{2} \mathrm{H}_{2}$ concentrations, which in turn lead to a lower soot surface HACA growth rate than in the $\mathrm{C}_{2} \mathrm{H}_{6}$ and $\mathrm{C}_{3} \mathrm{H}_{8}$ mixing cases. The slight increase in PAH concentration with $\mathrm{CH}_{4}$ mixing could not compensate for this reduction in surface growth rate and, finally, a decrease in soot loading resulted. It is worthwhile to contrast this monotonic decrease of SVF in CDFs to an earlier investigation using coflow experiments, where a notable synergistic effect of ethylene and methane mixture was observed on peak centerline soot volume fraction [668]. Although the difference may have been caused by the different time-temperature histories encountered between the counterflow and coflow configurations, it was noted that in the coflow study [668] the fuel was progressively less diluted with $\mathrm{N}_{2}$ from the neat $\mathrm{C}_{2} \mathrm{H}_{4}$ to the neat $\mathrm{CH}_{4}$ case, so that the different level of dilution may also play a role. More studies are required to unravel this discrepancy.

The special role of $\mathrm{C}_{2} \mathrm{H}_{4}$ was rather evident, as it was involved in all the above cases that exhibited synergistic effects in binary fuel mixtures. McEnally and Pfefferle [642] insightfully noted that since it is not easy for $\mathrm{C}_{2} \mathrm{H}_{4}$ to decompose to $\mathrm{CH}_{3}$, the sooting characteristics of $\mathrm{C}_{2} \mathrm{H}_{4}$ flame are particularly sensitive to $\mathrm{CH}_{3}$ produced from the dopants. As a matter of fact, $\mathrm{C}_{2} \mathrm{H}_{4}$ (along with $\mathrm{C}_{2} \mathrm{H}_{2}$ ) is special, since most other common alkane and alkene fuels can easily form $\mathrm{CH}_{3}$ in the fuel pyrolysis process. It can thus be reasoned that no $\mathrm{CH}_{3}$-induced synergistic effect may be developed for flames of base fuels other than $\mathrm{C}_{2} \mathrm{H}_{4}$ and $\mathrm{C}_{2} \mathrm{H}_{2}$. Indeed, with an increasing amount of DME $\left(\mathrm{CH}_{3}-\mathrm{O}-\mathrm{CH}_{3}\right.$, an effective source of $\mathrm{CH}_{3}$ radical) was added to $\mathrm{CDFs}$ of $\mathrm{CH}_{4}, \mathrm{C}_{2} \mathrm{H}_{6}$ and $\mathrm{C}_{3} \mathrm{H}_{8}$, the peak soot volume fraction and PAH LIF signals showed only a trend of monotonic decrease [100], which can be understood since all of the above base fuels can-by themselves-produce appreciable amounts of $\mathrm{CH}_{3}$ and thus are not sensitive to additional $\mathrm{CH}_{3}$ from DME decomposition. One notable exception that cannot be explained by this reasoning is the synergistic effects of methane and ethane mixtures, as observed in coflow diffusion flames of Trottier et al. [665]. However, considering that no quantitative soot volume fraction data or kinetic explanation was provided in Ref. [665] with respect to this fact, it 
may be interesting to perform additional experimental and kinetic modelling studies to confirm such a trend in CDFs.

As demonstrated above, non-monotonic variation of $\mathrm{PAH}$ and soot concentrations with mixing ratio have been widely observed in both counterflow and coflow [71, 284] diffusion flames of ethylene-based binary fuel mixtures. However, the situation in premixed flames can be drastically different. For instance, Wu et al. [103] measured PAH and soot in rich premixed flames of ethyleneethanol mixtures and observed that ethanol addition monotonically decreased soot formation. Similar observations were made by Salamanca et al. [607] for ethylene-ethanol, and by Sirignano et al. [90] for ethylene-DME premixed flames. These interesting results suggest that physicochemical pathways of PAH/soot formation for the same fuels can differ notably, depending on flame conditions. A hint of such dependence (in particular, the presence (absence) of the synergistic effects of ethylene-based binary fuels in non-premixed (premixed) flames) may be found in the results from partially-premixed CDFs. Hwang et al. [165] found that the non-monotonic variation of soot volume fraction in CDFs of ethylene-propane mixtures can be mitigated-and even eliminated-by premixing the fuel stream with oxygen. The explanation lay in the fact that with an oxidizer present in the fuel stream, the oxygen atom may react in the fuel pyrolysis zone with $\mathrm{C}_{2} \mathrm{H}_{2}$ to form $\mathrm{CH}_{2}$ radical $\left(\mathrm{C}_{2} \mathrm{H}_{2}+\mathrm{O}=\mathrm{CH}_{2}+\right.$ $\mathrm{CO}$ ), which in turn interacts with $\mathrm{C}_{2} \mathrm{H}_{2}$ to form the important benzene precursor, propargyl radical $\left(\mathrm{C}_{2} \mathrm{H}_{2}+\mathrm{CH}_{2}=\mathrm{C}_{3} \mathrm{H}_{3}+\mathrm{H}\right)$. In such a way, the bottleneck of benzene formation, caused by the deficiency of $\mathrm{C}_{1}$ species in neat $\mathrm{C}_{2} \mathrm{H}_{4}$ flame, can be effectively removed and as a result, the partiallypremixed $\mathrm{C}_{2} \mathrm{H}_{4}$ flame would not be as sensitive to additional $\mathrm{CH}_{3}$ from propane decomposition, mitigating the synergistic effect. Although yet to be confirmed, the monotonic decrease in soot concentration in ethylene-ethanol [103] and ethylene-DME premixed flames [90] is thought to be explainable by this mechanism. 


\section{c) Surrogates for practical fuels}

Practical fuels are complex mixtures of hundreds of hydrocarbons [656, 669-672] that prohibit kinetic simulation with detailed chemistry models. Therefore, simplified surrogates with a relatively small number of components are needed to represent various physical and chemical properties of real fuels. As a critical fuel property, the sooting tendency is an important consideration in surrogate development $[673,674]$, rationalizing the wide interest in the compositional effects of surrogate fuels on soot formation. Often the studies of surrogate fuels were performed by doping a small amount of the surrogate into the baseline flames of simple hydrocarbons (i.e., methane or ethylene), so that the compositional effect could be better isolated from other non-chemical factors, such as flame temperature and flow fields $[123,675,676]$. In addition, the risk of fuel vapor re-condensation can be minimized since the partial pressure of a target fuel may be rather small [677]. This point is particularly important for fuel components with a high molecular weight, and thus, a high boiling temperature.

With in-flame gas sampling and GC/MS analysis, Bufferand et al. [677] compared the detailed chemical structures of methane counterflow diffusion flames doped with $1000 \mathrm{ppm}$ of real jet fuel and a corresponding six-component surrogate (Utah/Yale surrogate). With highly-diluted non-sooting flames to facilitate probe sampling, the experimental data showed that the jet fuel-doped flame produced notably more benzene and toluene (more than a factor of two) than the surrogate-doped flame, although the surrogate was seen to capture other general jet fuel behavior reasonably well. In a follow-up work, Jahangirian et al. [80] investigated incipiently sooting ethylene counterflow diffusion flames doped with $2000 \mathrm{ppm}$ of jet fuel and two surrogates (six-component Utah/Yale surrogate [677] and two-component Aachen surrogate). Good agreement was observed between the real fuel and the surrogates in terms of peak location and magnitude of benzene concentration. With respect to toluene formation, the Utah/Yale surrogate performed better in mimicking the behavior of 
real jet fuel. Nevertheless, it was concluded that both surrogates exhibited acceptable agreement with real fuel in terms of aromatic formation under incipient sooting conditions.

Realizing the lack of fundamental studies in non-premixed flames of critical transport/surrogate fuel components, Carbone and Gomez conducted a series of investigations on the chemical structures of CDFs doped with hundreds of ppm of toluene [606], 1,2,4-trimethyl benzene (TMB) [609], ndecane [617] and n-decane / TMB mixtures [621]. In these studies, two baseline flames (i.e., a nonsooting methane SFO flame and an incipiently sooting ethylene SF flame) with significantly different thermal-chemical environments were used as flame reactors to provide well-defined (and contrasting) environments for the examination of reaction pathways of the target fuels. Profiles of flame temperature and mole fractions of important intermediate species were measured with thermocouples and GC/MS techniques, respectively. Analysis of the experimental data, together with numerical results obtained with literature-based kinetic mechanisms, also provided direction for future improvement of chemical models, especially in the application of non-premixed flame coupling kinetics and transport.

Choi et al. [622] doped n-heptane, benzene, and toluene separately, up to 20\% (molar ratio) in sooting ethylene $\operatorname{CDF}\left(X_{\mathrm{F}, 0}=1.0, X_{\mathrm{O}, 0}=0.3\right)$, and quantified the effects of these dopants on PAH and soot formation with the techniques of LIF and LII, respectively. The addition of benzene and toluene was confirmed to enhance soot/PAH formation, with toluene exhibiting stronger effects, which was consistent with the higher sooting tendency of toluene [678]. Kinetic analyses revealed that in toluene-doped flames, the formation pathway for large PAHs from benzyl radicals through indenyl

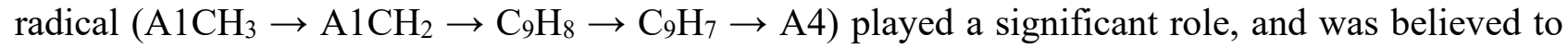
partially explain the higher soot loading of toluene.

With similar measurement techniques, the sooting characteristics of n-heptane, iso-octane and toluene, components of the widely-used toluene primary reference fuel (TPRF), were studied in CDFs by Choi et al. [447]. Both SF and SFO flames were tested and the liquid fuels were diluted only by 
$\mathrm{N}_{2}$, without employing a base gaseous fuel. As can be seen in Figure 26, the fuel and oxidizer dilution levels for the SF flames were somewhat high $\left(X_{\mathrm{F}, 0}=0.30\right.$ and $\left.X_{\mathrm{O}, 0}=0.21\right)$, so the flame was nonsooting when neat $n$-heptane or iso-octane was the fuel. Soot loading was monotonically increased as more toluene was added, and the behavior was qualitatively similar between n-heptane/toluene and iso-octane/toluene mixtures. Note, however, that the effect of toluene addition only became significant when the toluene ratio $\left(R_{\mathrm{T}}\right)$ was larger than 0.4 . On the other hand, interesting nonmonotonic variations of PAH concentrations (represented by the intensities of PAH LIF signals) with $R_{\mathrm{T}}$ were observed in both SF and SFO flames, which were qualitatively captured by kinetic modelling using a mechanism based on the literature [679]. It was determined through analysis of the numerical results that, as $R_{\mathrm{T}}$ increased, the concentration of benzyl radical increased, while that of the $\mathrm{H}$ radical decreased, leading to non-monotonic variations of the rates for major benzene production reaction: $\mathrm{A}_{1} \mathrm{CH}_{3}+\mathrm{H}=\mathrm{A} 1+\mathrm{CH}_{3}$. In addition, ethynylbenzene $\left(\mathrm{A}_{1} \mathrm{C}_{2} \mathrm{H}\right)$ showed significant synergistic effects, while biphenyl radical (P2-) increased and $\mathrm{C}_{2} \mathrm{H}_{2}$ decreased with an increase in $R_{\mathrm{T}}$, resulting in the non-monotonic behavior of $\mathrm{A} 3$ through $\mathrm{A} 1-+\mathrm{A} 1 \mathrm{C}_{2} \mathrm{H}=\mathrm{A} 3+\mathrm{H}$ and $\mathrm{P} 2-+\mathrm{C}_{2} \mathrm{H}_{2}=\mathrm{A} 3+\mathrm{H}$, respectively. While this investigation [447] dealt with only binary mixtures, a follow-up study extended the work to ternary mixtures [629].

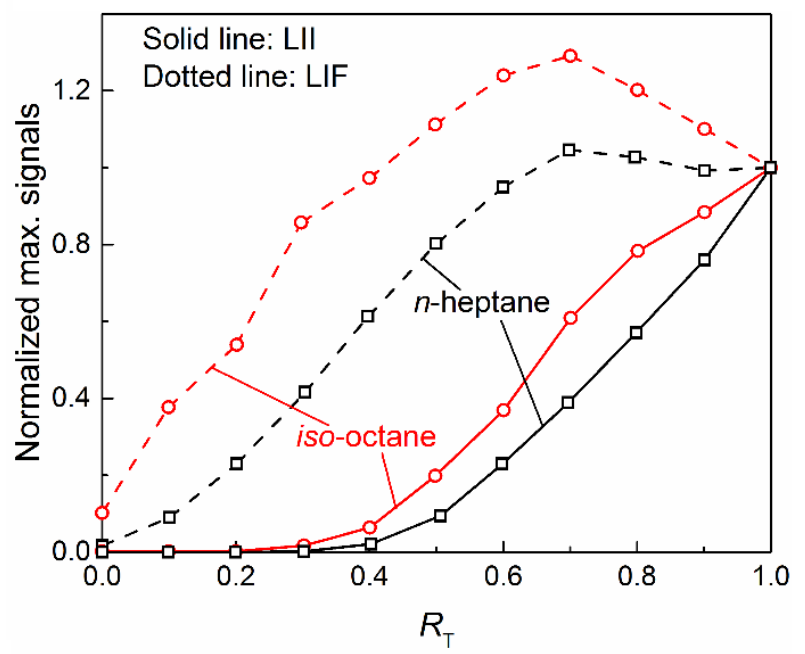

Figure 26. Normalized peak soot volume fraction (measured with LII) and PAH LIF signal as a function of toluene mixture ratio $\left(R_{\mathrm{T}}\right)$ in counterflow diffusion flames of $\mathrm{n}$-heptane/iso-octane and 
toluene mixtures. Fuel and oxidizer are both diluted with $\mathrm{N}_{2}$ and $X_{\mathrm{F}, 0}=0.30, X_{\mathrm{O}, 0}=0.2$. Reprinted from Choi et al. [447] with permission of Elsevier.

\subsubsection{Quantitative indices of sooting tendencies}

The above discussions demonstrate that fuel molecular structures are critical in determining soot production. This has important implications for the prediction of soot emissions from practical combustion devices which typically burn real fuels (i.e., diesel and jet fuels) with very complex compositions $[669,670]$. It is common for current predictive tools to use surrogates to represent real fuels $[669,670,673]$, making a quantitative index necessary to assess whether the sooting tendencies among them are matched [680]. A sooting index can also be helpful for comparing sooting tendencies among fuels with different functional groups, so that statistical information on the relation between fuel molecular structure and sooting tendency can be obtained [678, 681, 682]. This sub-section briefly reviews available sooting indices, with particular focus on the sooting temperature index (STI), which is based on sooting limits of counterflow diffusion flames.

Sooting metrics have been proposed for pyrolysis, premixed, and non-premixed combustion conditions. Crossley et al. [683] pyrolyzed $20 \mu \mathrm{L}$ of vaporized liquid samples in an alumina bed at $850^{\circ} \mathrm{C}$ and quantified the amount of carbon deposition with a temperature-programmed oxidation system. By normalizing the soot deposition of different fuel samples to two reference compounds, a micropyrolysis index (MPI) was proposed, which was believed to provide information on the tendency of a particular fuel to soot through oxygen-free pyrolysis (i.e., independent of heating value, oxidation chemistry, oxidizing environment, and etc.).

For premixed systems, critical equivalence ratios, or $\mathrm{C} / \mathrm{O}$ ratios at which soot luminosity can be visually detected, were frequently used as a quantitative metric [119, 645, 684]. Considering that under fuel-rich conditions, fuel carbon is converted primarily to $\mathrm{CO}$ instead of $\mathrm{CO}_{2}$, a critical effective equivalence ratio was also proposed [685], based on the stoichiometric relation in which $\mathrm{CO}$ and $\mathrm{H}_{2} \mathrm{O}$ were the combustion products. 
Sooting tendencies in non-premixed systems have been historically quantified using smoke point, which is the height of the highest flame without soot leakage when the test fuel is burned in a wickfed lamp [686], or in a coflow burner (for gaseous fuels) [104, 117]. Laminar co-flow jet flames are characterized by a buoyancy-controlled velocity field so that the velocity at a given height above the burner is proportional to the square root of the height, while reasonably independent of the fuel outlet velocity or volumetric flow rate [66]. As a result, as the fuel flow rate (or outlet velocity) increases, the flame becomes longer, increasing the fuel residence time, which in turn enhances the sooting propensity of the flame. There exists a critical flame height above which soot formed in the fuel-rich region of the flame cannot be fully oxidized and smoke begins to pierce from the flame front to emit into the environment. This critical flame height is typically defined as the smoke point. Obviously, fuels with a lower smoke point have a higher tendency to soot and there is a historical sooting index that was defined to be inversely proportional to the smoke point [687].

Quantitative determination of the critical flame height (i.e., the smoke point) is somewhat subjective and can vary from one instrument to another. Furthermore, as more oxygen is needed to form a stoichiometric fuel/oxidizer condition at the flame front higher molecular weight fuels naturally have longer flame [680], irrespective their sooting tendencies. In this regard, smoke point is affected by both the sooting tendency and the molecular weight of the fuel. In an effort to reduce this undesirable molecular weight effect, Calcote and Manos [645] introduced the threshold soot index (TSI), with a scale of 0 (ethane) to 100 (naphthalene), where higher values mean higher sooting propensity. This index is a normalization of smoke point data as given by:

$$
T S I=a\left(\frac{M W}{S P}\right)+b
$$

where MW is the molecular weight of the test fuel, SP is the smoke point and $a$ and $b$ are apparatusspecific constants. Note that by considering the linear relationship between flame height and fuel volumetric flow rate, $S P$ can also be replaced by the critical fuel volumetric flow rate in Eq. 24 for the definition of TSI. Despite its wide use, TSI is just one way among many to normalize smoke point 
data. Arguing that the direct inclusion of MW in sooting index definition is not physically motivated, Li and Sunderland [688] revisited smoke point data and proposed a new sooting metric called the normalized smoke point (NSP). Barrientos et al. [681] proposed the oxygen extended sooting index (OESI) for oxygenated fuels to account for the effect of oxygen atoms in the normalization of smoke point data.

Another sooting index that received widespread attention is the yield sooting index (YSI), as pioneered by McEnally and Pfefferle [689]. They doped target fuels in a methane coflow diffusion flame and measured the peak soot volume fraction, which was then normalized to determine YSI [67, $653,678,690,691]$. YSI was traditionally obtained by measuring SVF using the LII technique, and a more recent study [692] has shown that the techniques of color-ratio pyrometry and spectral soot emission are also compatible. In addition, measured YSI values were also unvarying across flame conditions with different temperatures and levels of partial premixing, suggesting that the robustness of YSI to be used across a range of premixed and temperature conditions is relevant to soot formation [692].

The above indices (except the MPI) are based on either coflow or smoke point flames in which undiluted fuel and air were typically used. Recent research in novel combustion techniques such as oxygen-enriched combustion, low temperature combustion and flameless combustion [693] necessitates the study of the effects of fuel and oxidizer stream dilution on sooting characteristics of diffusion flames. Furthermore, considering soot formation is a rate-limiting process [554], and therefore sensitive to the characteristic flow time scale, it would be ideal if the effects of residence time are reflected in the sooting index. In coflow diffusion flames, the residence time is typically too long to represent combustion under practical conditions [114]; challenges also exist for its active control. In addition, as discussed in a previous section, in coflow flames, soot once formed will be convected toward the oxidizing zone where soot oxidation takes place, behaving as a soot formation/oxidation (SFO) flame. In such a case, the smoke point is essentially a critical condition 
when soot formation and oxidation are balanced. However, to quantify the sooting tendency for various fuels, it may be helpful to avoid the interference from soot oxidation.

$\mathrm{CDF}$ is a flame configuration that can provide valuable information regarding residence time effects on soot formation; because it is relatively easy to parameterize the strain rate (inversely proportional to residence time) by adjusting the nozzle exit velocities. Also, by utilizing SF flames, it is possible to separate the soot formation process from oxidation. The sooting tendencies of different fuels have been systematically studied in CDFs in the form of sooting limit curves [620]. An example sooting limit map is shown in Figure 27 for various $\mathrm{C}_{1}-\mathrm{C}_{4}$ fuels. A CDF can be manipulated to be sooting or non-sooting by varying the amount of $\mathrm{N}_{2}$ dilution in the fuel or oxidizer stream (detailed discussion on dilution effect on soot formation are presented in Section 5.2). Experimentally, whether a CDF is sooting can be determined by comparing the Rayleigh and Mie scattering signals from gas molecules and soot particles, respectively. As detailed in Ref. [614], under non-sooting conditions the Raleigh scattering intensity varies monotonically from the fuel to the oxidizer stream due to the change in the scattering cross-section of gases. Once soot is formed, the Mie scattering signal from soot is embedded in the Rayleigh signal, resulting in a local maximum. The presence of this local maximum is indicative of the onset of soot.

It was shown in Ref. [614] that in an ethylene CDF with $X_{\mathrm{F}, 0}=1.0$, soot formation could only be observed when $X_{\mathrm{O}, 0}$ was higher than 0.18 . As such, $X_{\mathrm{F}, 0}=1.0$ and $X_{\mathrm{O}, 0}=0.18$ can be regarded as a critical condition for soot formation. Similarly, a corresponding critical $X_{\mathrm{O}, 0}$ always exists for a specified $X_{\mathrm{F}, 0}$. In this regard, a sooting limit curve can be drawn in the $X_{\mathrm{F}, 0}-X_{\mathrm{O}, 0}$ plane by connecting the critical sooting limit points $\left(X_{\mathrm{F}, \mathrm{cr}}, X_{\mathrm{O}, \mathrm{cr}}\right)$ as shown in Figure 27. 


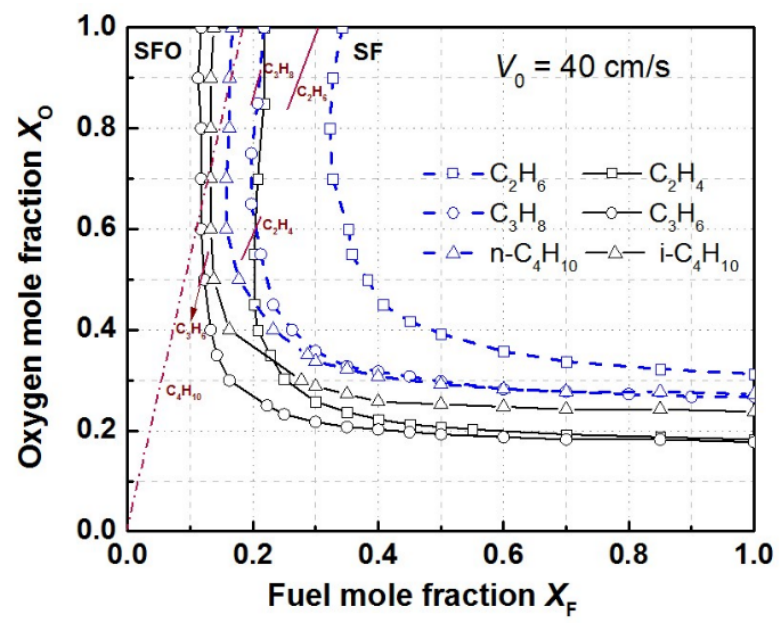

Figure 27. Sooting limit map of various $\mathrm{C}_{1}-\mathrm{C}_{4}$ fuels in counterflow diffusion flames, measured with nozzle exit velocity at $40 \mathrm{~cm} / \mathrm{s}$. Reprinted from Wang and Chung [620] with permission of Elsevier. Brown dotted line represents theoretical SF-SFO flame boundaries.

These sooting limit curves divide the $X_{\mathrm{F}, 0}-X_{\mathrm{O}, 0}$ plane into two regions: The upper right is the sooting region, and the remainder is the non-sooting region. Any combination of $X_{\mathrm{O}, 0}$ and $X_{\mathrm{F}, 0}$ in the upper right region will result in a sooting flame. It is clear from Figure 27 that the sooting regions have different sizes for different fuels. For example, the sooting region for $\mathrm{C}_{2}-\mathrm{C}_{3}$ alkene are noticeably larger than their alkane counterparts and iso-butane has a more extended sooting zone than n-butane. These are consistent with the known fact that, in diffusion flames, alkenes are generally more sooting than alkanes [99], and branched alkanes are more sooting than those with straight chains (with the same carbon number) [688]. A more interesting observation can be made when re-plotting the sooting limit data shown in Figure 27 as a function of calculated flame temperature $T_{\mathrm{f}}$. The results are shown in Figure 28. It can be seen that for SF flames (i.e., large $X_{\mathrm{F}}$ ), the flame temperature remains nearly fixed over a wide range of $X_{\mathrm{F}, \mathrm{cr}}$, indicating the existence of a threshold temperature for the onset of soot formation in SF flames. The flame will be sooting if it has a peak temperature above this threshold, and vice versa. What is more important is that the threshold temperature is highly dependent on the fuel type, with less sooting fuel featuring a higher threshold temperature at the sooting limit. 


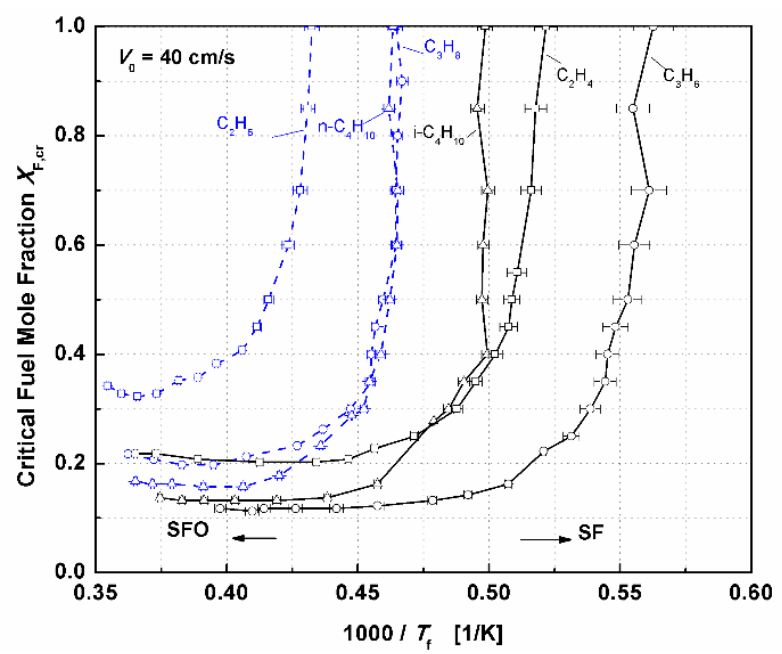

Figure 28. Critical fuel mole fractions at sooting limit conditions plotted as a function of calculated flame temperature for various $\mathrm{C}_{1}-\mathrm{C}_{4}$ fuels in counterflow diffusion flames with nozzle exit velocity at $40 \mathrm{~cm} / \mathrm{s}$. Reprinted from Wang and Chung [620] with permission of Elsevier.

In fact, this dependence of the threshold temperature for SF flame with $X_{\mathrm{F}, 0}=1.0\left(T_{\mathrm{cr} ; \mathrm{SF}}\right)$ on fuel type provides an alternative means for ranking the sooting tendency of different fuels. To demonstrate and validate the possibility for its use as a quantitative sooting index, the correlation between $T_{\mathrm{cr} ; \mathrm{SF}}$ and the threshold soot index (TSI) is presented in Figure 29, where good correlation can be seen. This suggests that $T_{\mathrm{cr} ; \mathrm{SF}}$ determined from counterflow experiments can be a viable metric for sooting propensity. Note that the uncertainties of determining $T_{\mathrm{cr} ; \mathrm{SF}}$ are considerably lower than that for TSI. 


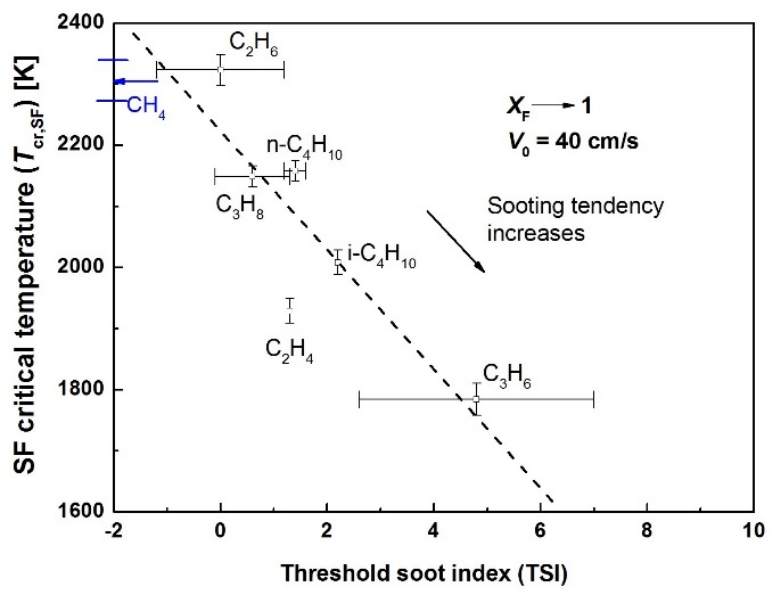

Figure 29. Correlation between threshold temperature for soot formation in SF flames and literature data of TSI. Reprinted from Wang and Chung [620] with permission of Elsevier.

By normalizing $T_{\mathrm{cr} ; \mathrm{SF}}$ of the test fuels to two reference fuels in a similar manner as used for YSI, a sooting temperature index (STI) can be defined. This CDF-based sooting index has the following novel features compared to smoke point-based ones: 1) It quantitatively represents the limiting conditions of soot onset for a target fuel, while smoke point represents a condition where soot loading is too excessive to be fully oxidized; 2) In the test environment for SF flames, interference from soot oxidation is absent, so the sooting tendency can be assessed purely on the fuel's chemical reactivity to form soot; 3) The effect of residence time can easily be incorporated quantitatively in STI, by measuring sooting limits with different nozzle exit velocities. A sooting sensitivity index (SSI) was also proposed by normalizing the slope of $T_{\mathrm{cr} ; \mathrm{SF}}$ as a function of nozzle exit velocity $\left(\mathrm{d} T_{\mathrm{cr}, \mathrm{SF}} / \mathrm{d} V_{0}\right)$, and it was found that the sooting tendency of different fuels also has different sensitivity to variations in residence time [620].

Finally, it may also be noteworthy that the approach used to develop YSI [689] (i.e., doping a small amount of test fuel in a base flame and measure the peak SVF) can also be applied to CDF, and further efforts are needed to quantify the dependence of sooting tendency data especially on pressure. 


\subsection{Effects of dilution and non-hydrocarbon additives on soot formation}

The identification of soot-inhibiting non-fuel additives is an important research area for practical purposes [564]. For example, flue gas recirculation (FGR), which is essentially the addition of $\mathrm{CO}_{2}$ and $\mathrm{H}_{2} \mathrm{O}$ in reactants, is frequently used in utility boilers as a measure for emission reduction [694]. Similarly, exhaust gas recirculation (EGR) is widely adopted in internal combustion engines. Oxyfuel combustion is also actively explored to reduce the cost of $\mathrm{CO}_{2}$ sequestration. It is of fundamental interests to understand the underlying mechanisms on how these additives affect soot formation. Typical non-hydrocarbon additives include inert gases such as nitrogen, argon, helium, carbon dioxide, and water vapor, as well as oxidizing agents of oxygen (i.e., partial-premixing), ozone, and nitrogen dioxide. The influences of organometallic and halogenated compounds on soot formation have also attracted research interests. In this section, relevant studies conducted with laminar counterflow flames are reviewed and discussed.

\subsubsection{Nitrogen and noble gas addition}

The effect of nitrogen dilution in oxidizers was studied by Vandsburger et al. [94] in counterflow diffusion flames of ethylene and propane, stabilized in the forward stagnation region of a cylindrical porous burner. The level of oxidizer dilution was quantified by $X_{\mathrm{O}, 0}$. The flames were comprehensively characterized; the profiles of axial velocity (LDV), temperature (thermocouples), and soot volume fraction, number density, and average diameter (light extinction and scattering) were all measured along the stagnation streamline. The results showed that for ethylene flames, the increase in $X_{\mathrm{O}, 0}$ from 0.18 to 0.28 resulted in increases in the maximum flame temperature $(1750 \mathrm{~K}$ to 2150 $\mathrm{K}$ ) and peak soot volume fractions (from $3.7 \times 10^{-7}$ to $2.1 \times 10^{-6}$ ). Maximum particle number densities (maximum particle sizes) were measured to occur close to the flame front (stagnation plane), and increased slightly with $X_{\mathrm{O}, 0}$. Propane flames exhibited similar behavior with respect to the effect of $X_{\mathrm{O}, 0}$, although soot loading was notably lower compared to ethylene. Hwang and Chung [281] 
performed similar experiments in ethylene SF CDFs and found that both soot and PAH formation were enhanced with the increase in $X_{\mathrm{O}, 0}$. In a more recent work [614], the range of $X_{\mathrm{O}, 0}$ investigated was extended further, up to 0.5 for ethylene $\operatorname{CDF}\left(X_{\mathrm{F}, 0}=1\right)$, and similarly, a monotonic increase in soot loading with increased $X_{\mathrm{O}, 0}$ was observed. In a counterflow diffusion flame with neat methane as the fuel stream, Beltrame et al. [576] increased $X_{\mathrm{O}, 0}$ from 0.21 all the way to 1.0 (pure oxygen). Their experimental and numerical results agreed, showing a monotonic increase in peak soot volume fraction. As a related fact, it is interesting that Kennedy and coworkers also observed the formation of multi-walled carbon nanotubes [506, 695], as well as fullerenes [696] in methane or acetylene CDFs with oxygen-enriched oxidizer.

At first glance, it is intriguing that an increase in oxygen content in the oxidizer stream (i.e., $X_{\mathrm{O}, 0}$ ) would strengthen soot formation. It might seem that the increased amount of oxygen would enhance soot oxidation, and therefore, result in lower soot production. This can be true for over-ventilated coflow diffusion flames, where the final soot loading is determined by competing effects of soot production in the fuel-pyrolysis zone, and subsequent soot oxidation in the oxygen-rich post-flame zone. For example, Glassman and Yaccarino [697] found that the sooting tendency of ethylene coflow diffusion flame (represented by the normalized sooting flame height) decreased as $X_{\mathrm{O}, 0}$ increased from 0.16 to 0.24 , which was explained by the fact that higher $X_{\mathrm{O}, 0}$ resulted in a higher soot burn-up rate. However, all the counterflow flames mentioned above are of the SF type, so that the flame was stabilized on the oxidizer side of the stagnation plane. As discussed in a previous section, in such cases the soot particles would be transported away from the oxidizing flame sheet so that soot oxidation was largely absent. Higher $X_{\mathrm{O}, 0}$ would lead to higher temperatures in the reacting flame front as well as in the fuel pyrolysis zone, enhancing fuel pyrolysis and soot formation rates [93, 698].

The effect of fuel stream dilution by inert gases has also been studied. Du et al. [568] measured the critical strain rate for soot inception (i.e., soot inception limit, $K_{\mathrm{cr}}$ ) in $\mathrm{CDFs}$ of $\mathrm{C}_{2} \mathrm{H}_{4}, \mathrm{C}_{3} \mathrm{H}_{8}$, and n$\mathrm{C}_{4} \mathrm{H}_{10}$ with fuel side dilution by nitrogen and argon. The data for the $\mathrm{C}_{2} \mathrm{H}_{4}$ flame is re-plotted in Figure 
30, and other flames exhibit similar qualitative trends. As can be seen, and much as expected, $K_{\text {cr }}$ decreased (i.e., sooting tendency decreased) monotonically with the increase in the dilution level. Considering that inert dilution can affect soot formation by variation of either the flame temperature or the concentration of soot precursors, additional efforts were taken to isolate these effects. In Figure $30 \mathrm{~b}$, the dashed line represents the variation of $K_{\mathrm{cr}}$ with flame temperature, which was varied by substituting part of the nitrogen in the oxidizer with equal moles of argon, without altering the fuel/oxidizer dilution level [699]. In such a way, this line depicts only the thermal effect. The symbols are data shown in Figure 30a, re-plotted as a function of temperature. Its deviation from the dotted line indicates the inert affecting soot inception via not only thermal, but dilution effects. Indeed, the decrease in $K_{\mathrm{cr}}$ with $\mathrm{N}_{2} /$ Ar addition was faster than expected from only thermal considerations. At a specified temperature, $K_{\mathrm{cr}}$ (in cases with argon dilution), was even lower than those with $\mathrm{N}_{2}$ dilution. This can be explained by the fact that more argon is required than nitrogen for the temperature to be matched, due to the lower specific heat of argon, so the argon diluted flame has a higher level of dilution. The importance of concentration modification in explaining the reduction of soot by inert addition was also noted in experiments of jet flames [96, 700]. 

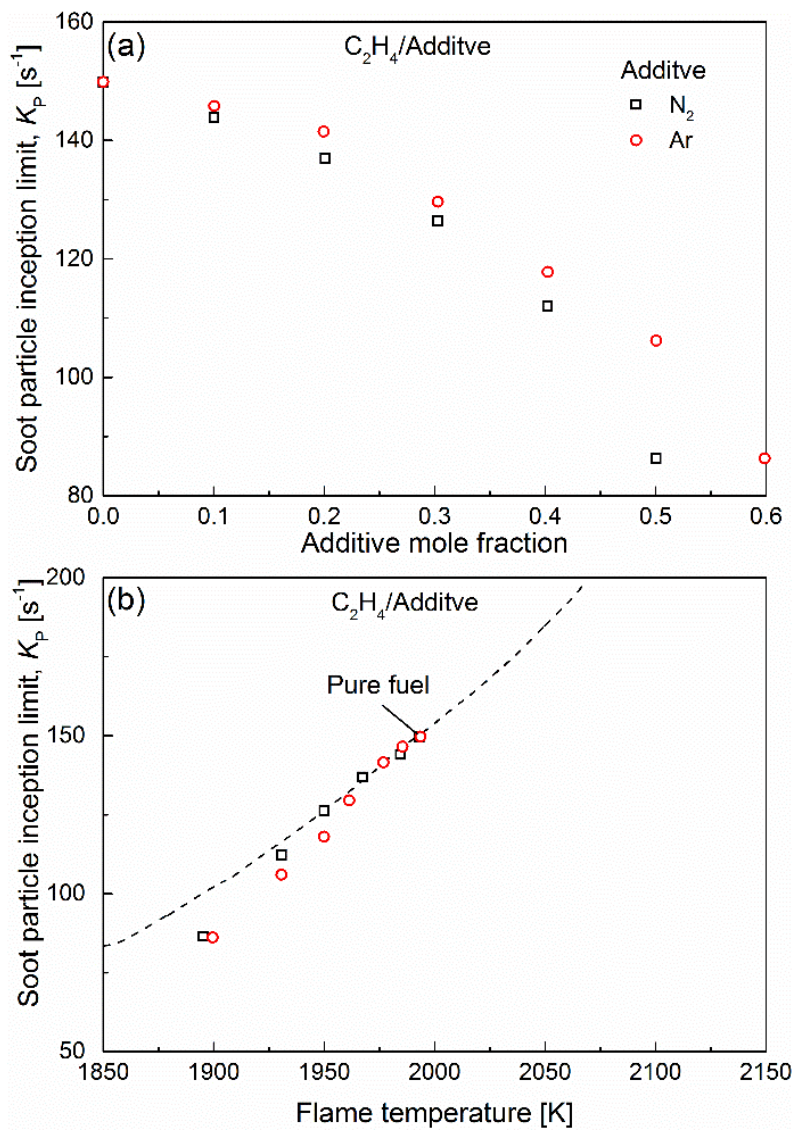

Figure 30. Critical strain rates for soot formation in ethylene CDFs with fuel side $\mathrm{N}_{2}$ and Ar dilution (a) and effects of temperature on soot inception (b). Replotted with data taken from Du et al. [568].

In addition to $K_{\mathrm{cr}}$, sooting characteristics were also compared carefully in moderately sooting CDFs, with and without fuel stream inert addition [74]. Soot volume fraction, axial velocity and temperature profiles were experimentally determined for ethylene CDFs with $X_{\mathrm{F}, 0}=1.0,0.75$ and 0.5 for $X_{\mathrm{O}, 0}=$ 0.21. The dilution and temperature effects were isolated in additional experiments with manipulation of $\mathrm{N}_{2} / \mathrm{Ar}$ ratio in the oxidizer side, similar to [568]. The data shown in Figure 31, with fixed temperatures, clearly demonstrate the notable effects of concentration modification on soot formation. Note, in Figure 31, the SVF was plotted against convective time from the flame front to account for the fuel stream inert addition that would alter the flame position, and thus the residence time of soot particles. A further analysis of the slope of SVF with time indicated that the soot production rate varied linearly with initial fuel concentration $X_{\mathrm{F}, 0}$, provided that the flame temperature remained constant. 


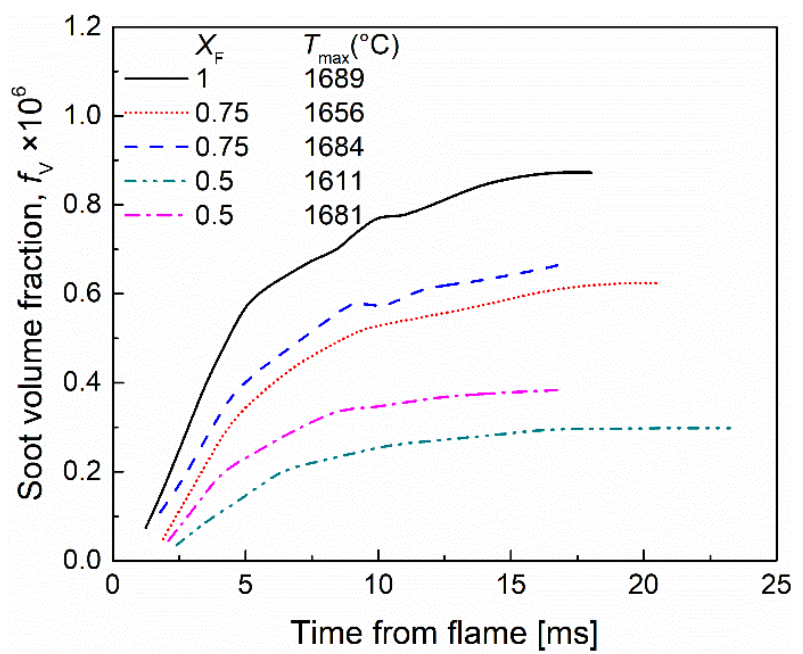

Figure 31. Separation of temperature and dilution effects on soot formation in ethylene CDFs, data taken from Axelbaum et al. [74].

As previously discussed, sooting limits in CDFs can be represented by the critical strain rate $K_{\mathrm{cr}}$, beyond which soot scattering signals disappear at a fixed $X_{\mathrm{F}, 0}$ and $X_{\mathrm{O}, 0}$ [551]. Because of the effects of inert addition in inhibiting soot formation, it may also be represented by the critical $X_{\mathrm{F}, 0}$ and $X_{\mathrm{O}, 0}$, below which soot cannot form altogether at a fixed strain rate. Indeed, a CDF can be manipulated to be sooting or non-sooting by varying the amount of $\mathrm{N}_{2}$ dilution in the fuel or oxidizer stream. As mentioned before, a sooting map can be drawn in the $X_{\mathrm{F}, 0}-X_{\mathrm{O}, 0}$ plane, and a further example (obtained by Joo et al. for ethylene fuel [281]) can be seen in Figure 32a. The critical sooting conditions (black squares) in terms of $X_{\mathrm{F}, 0}$ and $X_{\mathrm{O}, 0}$, were determined from scattering signals where soot signals are about to appear, similar to the description in [551]. In subsequent studies, similar soot maps were obtained for various other fuels and fuel mixtures $[614,620]$, which were then used to compare the sooting tendency of these neat, or mixture fuels. 

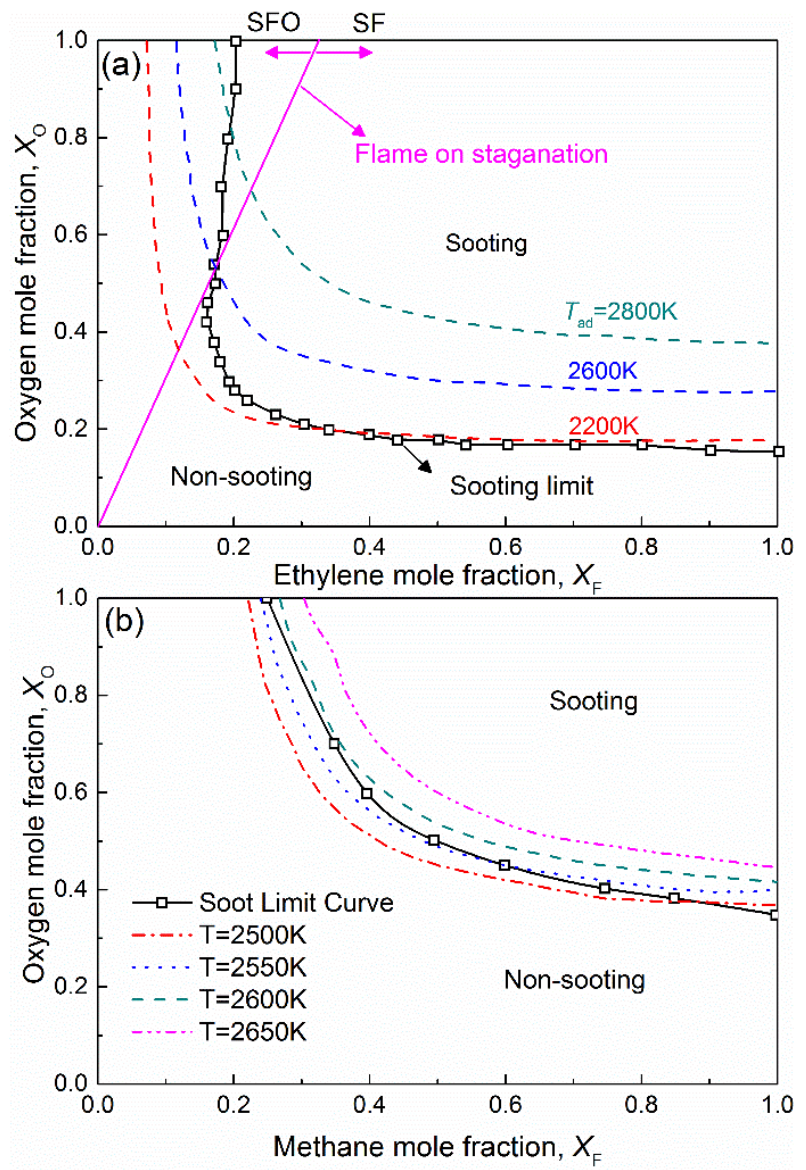

Figure 32. Sooting limit curve of counterflow diffusion flames as determined with light scattering technique (a), and with visual inspection (b). Data taken from Refs. [281] and [581] for (a) and (b), respectively.

Instead of using light scattering, Naik et al. [581] determined the critical sooting condition based on visual inspection of the flame, so that the first observation of yellow soot luminosity against a darkened background was deemed to be a signal for incipient soot formation. Figure $32 \mathrm{~b}$ shows the soot map, developed with this visual criterion for methane CDFs at a constant global strain rate $K_{\mathrm{G}}$ of $20 \mathrm{~s}^{-1}$. A notable difference from light scattering-based measurements lies in the higher $X_{\mathrm{O}}$ region, where the obvious bending behavior, observed in the light scattering-based sooting limit curve (a) is absent in the visually-based curve (b). Although the two curves were measured for different fuels, later light scattering-based experiments [620] confirmed that the phenomena of bending in sooting limit curves are ubiquitous, and the bending is even stronger for low sooting tendency fuels like methane. 
Before explaining this discrepancy, it is helpful to identify several important features exhibited by the sooting limit curve shown in Figure 32a: 1) Beginning from the condition of $X_{\mathrm{F}, 0}=1.0$, the critical $X_{\mathrm{O}, 0}$ for soot onset increases only slightly as $X_{\mathrm{F}, 0}$ decreases down to a value of about 0.4 , beyond which the critical $X_{\mathrm{O}, 0}$ increases sharply. Such dramatic variations indicate that sooting behaviors in the large and small $X_{\mathrm{F}, 0}$ regions are rather different, caused by the transitions of flame/sooting structures. This is consistent with the distinction between SF and SFO flames, mentioned previously; 2) In SF flames with large $X_{\mathrm{F}, 0}$, the sooting limits closely follow the iso-temperature curve, suggesting that incipient soot formation is controlled by temperature. On the other hand, in SFO flames with low $X_{\mathrm{F}, 0}$ and large $X_{\mathrm{O}, 0}$, sooting limits span an appreciable range of iso-temperature curves, implying that temperature is not the only factor controlling soot formation in SFO flames; 3) The most interesting observation is, in fact, the bending behavior of the sooting limit curve of Figure $32 \mathrm{a}$, which can be crossed twice by a vertical line of constant $X_{\mathrm{F}, 0}$ (e.g., the line of $X_{\mathrm{F}, 0}=0.22$, not drawn). This has important implications in that for flames with a fixed $X_{\mathrm{F}, 0}$, as $X_{\mathrm{O}, 0}$ increases, the initial non-sooting flame can have a transition to the sooting regime and then become non-sooting again as $X_{\mathrm{O}, 0}$ approaches unity, exhibiting a non-monotonic behavior with $X_{\mathrm{O}, 0}$. Indeed, soot volume fraction measurements and accompanying numerical modelling results confirmed this non-monotonic influence of oxidizer composition on soot formation in CDFs with low $X_{\mathrm{F}, 0}$ [378]. In particular, it was experimentally shown that for ethylene CDF with $X_{\mathrm{F}, 0}=0.28$ and an effective strain rate $K_{\mathrm{E}}$ of 100 $\mathrm{s}^{-1}$, the peak SVF first increased — reaching a maximum near $X_{\mathrm{O}, 0}=0.7$-and then decreased as $X_{\mathrm{O}, 0}$ increased from 0.7 to 1.0 [378]. Further kinetic analyses revealed that, while increasing $X_{\mathrm{O}, 0}$ suppresses soot inception and enhances soot oxidation (tending to reduce soot production), soot surface growth rates are enhanced with the increase in $X_{\mathrm{O}, 0}$, contributing positively to soot mass concentration. It is these competing effects that lead to the non-monotonic variation of the sooting tendency with $X_{\mathrm{O}, 0}$ in SFO flames. Since the visually-based sooting limit curve shows no sign of bending (implying simply a monotonic dependence), it can be concluded that visual inspection may 
not be suitable for judging the onset of soot — at least for CDFs of SFO types. This is consistent with the findings of Du et al. [551], that the sooting limits determined by visual luminosity can be strongly affected by fuel type and flame geometry, and with Zhang et al. [525], who postulated that gaseous PAHs may also be responsible for the yellow color emissions in CDFs.

Compared to argon and nitrogen, helium has a special feature, that of high diffusivity. Since species diffusion is always coupled with chemical kinetics to determine general flame characteristics, it is reasonable to expect that the soot formation process would also be noticeably affected by preferential diffusion [701-703]. In this regard, the effects of helium addition on the sooting characteristics of diffusion flames have attracted wide research interests. For instance, McLintock [704] compared the effectiveness of adding various inert gases in an oxidizer stream in reducing the sooting propensity of ethylene coflow diffusion flames, and found helium to be most effective. However, in cases of fuel stream addition, Glassman and coworkers [705] noticed that helium was even less effective than argon in inhibiting soot formation of ethylene coflow flames. Axelbaum et al. [657] proposed an explanation for these interesting opposing trends based on concentration modification: When added to the fuel side, preferential diffusion effects will cause helium to defocus to a higher extent than argon, due to the concave curvature of the coflow diffusion flame front (i.e., negative stretch) [659]. As a result, concentrations of the fuel and other fuel-related intermediate species in the helium-diluted flame will be higher (effectively less diluted) than those in the argon-diluted flame, leading to higher soot formation in the helium-diluted flame. The opposite holds when the diluents are added to the oxidizer side, so that helium will be more focused, resulting in more effective dilution, and lowering the sooting tendency in the helium-diluted flame.

In addition to the effects of concentration modification, helium has a higher thermal diffusivity, so its addition can also cause a broadening of the temperature profile, which may thermally affect soot formation. By using a positively stretched flame, for which temperature broadening has the opposite effects (if any) to that of concentration modification on soot formation, Axelbaum et al. [657] 
provided unambiguous evidence to support the dominance of concentration modification as the underlying mechanism explaining the helium addition effects. In particular, noble gases such as helium, argon, neon, and krypton that have similar heat capacities, but progressively lower diffusivities, were added to the fuel stream (at a molar ratio of 0.2 ) of positively stretched CDFs. The results (Figure 33) showed that peak SVF was lowest in the case of helium addition for both ethylene and propane flames. Since temperature broadening due to the high thermal diffusivity of helium would tend to increase soot formation if effective, the experimental facts can only be explained by composition modifications. Note that the relative extent of the effects between fuel stream helium and argon dilutions were expected to be reversed as the signs of stretch rates changed. This is supported by later experimental investigations [706], where the fuel stream addition of hydrogen (a highly mobile gas, similar to helium) was found be less effective than $\mathrm{N}_{2}$ addition in reducing soot production in ethylene coflow flames, which can also be partly explained by this mechanism, especially considering that $\mathrm{H}_{2}$ has an additional chemical role in inhibiting soot formation in ethylene flames [662]. 

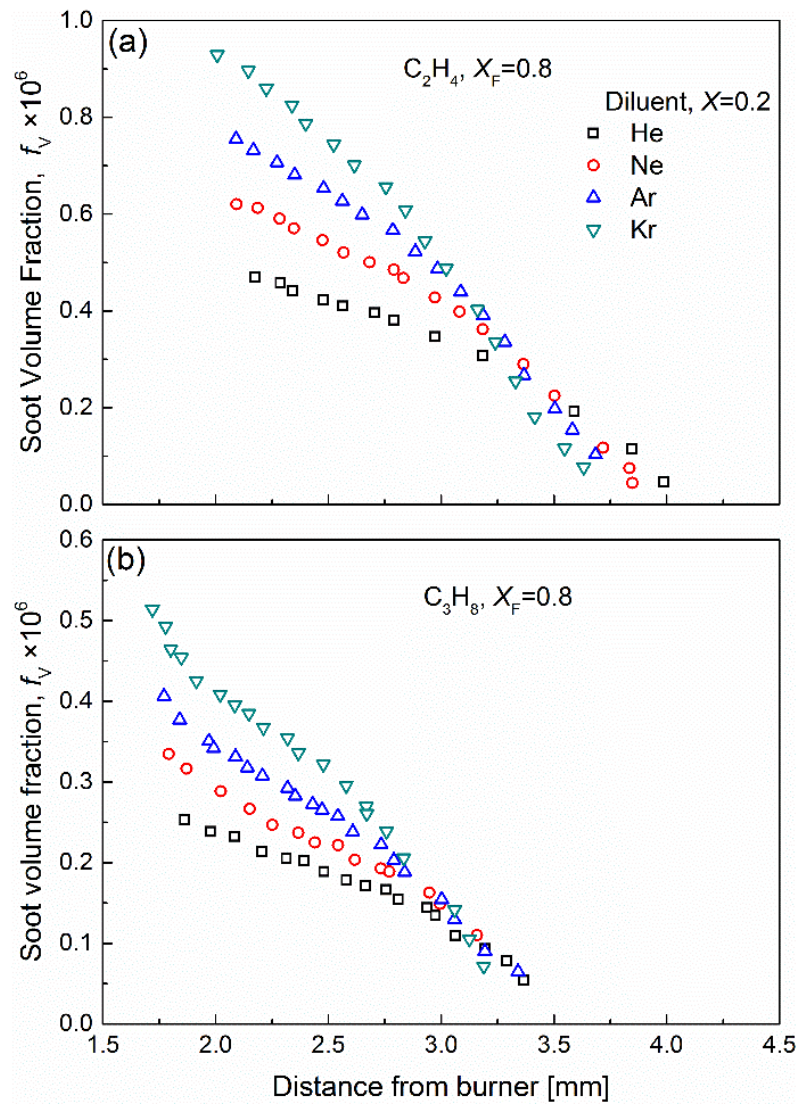

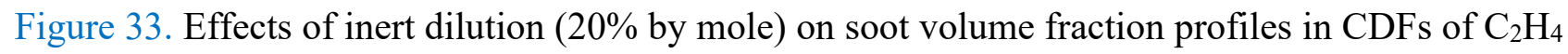
(a) and $\mathrm{C}_{3} \mathrm{H}_{8}$ (b). Data taken from Axelbaum et al. [657].

\subsubsection{Carbon dioxide and water vapor addition}

Although typically regarded as non-reactive, $\mathrm{CO}_{2}$ and $\mathrm{H}_{2} \mathrm{O}$ can participate in chemical reactions under high temperature flame conditions, so they could chemically affect fuel oxidation and pollutant formation processes. Detailed investigations on the effects of $\mathrm{CO}_{2}$ and $\mathrm{H}_{2} \mathrm{O}$ addition on soot formation can shed light on the underlying mechanism of these intricate chemical interactions (and help validate and improve existing kinetic models). Besides, the frequent use of exhaust/flue gas recirculation (EGR/FGR) techniques in practical combustion devices $[694,707,708]$ also motivates such studies.

Du et al. [564] experimentally determined the critical strain rate $K_{\mathrm{cr}}$ for soot inception in ethylene and propane $\mathrm{CDF}$ and found that soot formation was inhibited by $\mathrm{CO}_{2}$ addition in either the fuel or oxidizer side, for both flames. They also showed that $\mathrm{CO}_{2}$ addition inhibits soot formation via a combination of dilution, thermal, and chemical effects; although they did not identify detailed 
reaction pathways that are chemically affected by the addition of $\mathrm{CO}_{2}$. Zhang et al. [525] compared the experimentally measured $\mathrm{C}_{2} \mathrm{H}_{2}$ mole fraction, $\mathrm{SVF}$, and soot number density between a baseline ethylene CDF and one with $21 \% \mathrm{CO}_{2}$ addition on the fuel side, which have the same $X_{\mathrm{F}, 0}$ and $X_{\mathrm{O}, 0}$ and similar flame temperatures (via reactant preheating for the $\mathrm{CO}_{2}$ addition case). The data showed that $\mathrm{CO}_{2}$ addition resulted in notable reduction of $\mathrm{C}_{2} \mathrm{H}_{2}$ mole fraction and $\mathrm{SVF}$, attributed (through logical reasoning by the authors) to the fact that $\mathrm{CO}_{2}$ addition enhanced the reaction of $\mathrm{CO}_{2}+\mathrm{H}=$ $\mathrm{OH}+\mathrm{CO}$ to produce more $\mathrm{OH}$ radicals in the fuel pyrolysis zone, which then contributed to the oxidation of gas phase soot precursors. Annunziata et al. [709] measured the laser-induced emission (excited at $266 \mathrm{~nm}$ ) of ethylene CDFs and found the emissions detected both at 330/440 nm (termed LIF signals by the authors to represent nanoparticles constituted mainly by aromatic compounds) and at $550 \mathrm{~nm}$ (termed LII signal to represent soot aggregates of sizes higher than $10 \mathrm{~nm}$ ) were significantly reduced by the addition of $\mathrm{CO}_{2}$, either in the fuel or the oxidizer side, which were primarily attributed to the higher heat capacity of $\mathrm{CO}_{2}$, thereby the thermal effects.

To further clarify the chemically-inhibitive role of $\mathrm{CO}_{2}$ in soot formation, Liu et al. [710] performed numerical simulations with a detailed chemistry for ethylene CDFs. An innovative strategy was employed in the simulation, which used fictitious $\mathrm{CO}_{2}\left(\mathrm{FCO}_{2}\right)$, defined purely for numerical purposes, as a species that has the same thermodynamic/transport properties with real $\mathrm{CO}_{2}$ but does not participate in any chemical reactions. Chemical effects of $\mathrm{CO}_{2}$ addition were then identified by comparing the results obtained with $\mathrm{FCO}_{2}$ and those from with real $\mathrm{CO}_{2}$. The results indicated that the reduction in flame temperature, $\mathrm{C}_{2} \mathrm{H}_{2}$ concentration, and enhanced oxidative attacks by $\mathrm{O}$ and $\mathrm{OH}$ in the sooting zone, via $\mathrm{CO}_{2}+\mathrm{H}=\mathrm{OH}+\mathrm{CO}$ and $\mathrm{CO}_{2}+\mathrm{CH}=\mathrm{CO}+\mathrm{HCO}$, were the underlying mechanisms for the chemical role of $\mathrm{CO}_{2}$ in reducing soot formation. It was also found that adding $\mathrm{CO}_{2}$ to the oxidizer side was more effective in inhibiting sooting tendencies than in the fuel side. However, this study did not directly model PAH/soot formation, instead it used the changes of $\mathrm{C}_{2} \mathrm{H}_{2}$ mole fraction as an index to represent sooting tendency variations. Using the same $\mathrm{FCO}_{2}$ strategy, 
Wang and Chung [626] performed PAH-based soot modelling studies to determine the effects of fuel side $\mathrm{CO}_{2}$ addition on ethylene CDFs with detailed gas-phase chemistry and the method of moments to treat particle dynamics. After validation against the experimental data, numerical results showed that the addition of $\mathrm{CO}_{2}$ had chemical effects in: 1) reducing $\mathrm{PAH}$ mole fraction, which in turn decreased soot nucleation rates and number density, and thus lower surface area for mass-adding reactions, 2) decreasing hydrogen, methyl, and propargyl radicals, causing lower $\mathrm{H}$ abstraction rates in the production of active surface sites for soot growth, and 3) reducing $\mathrm{C}_{2} \mathrm{H}_{2}$ concentrations, and thus the HACA growth rates per unit surface area. They further measured the sooting limits in terms of critical $X_{\mathrm{F}, 0}$ and $X_{\mathrm{O}, 0}$ in $\mathrm{CO}_{2}$ atmosphere (i.e., both the fuel and oxygen are balanced by $\mathrm{CO}_{2}$ ) and the results are shown in Figure 34, with comparison against data measured in $\mathrm{N}_{2}$ atmosphere. It can be seen that the sooting region in the $X_{\mathrm{F}, 0}-X_{\mathrm{O}, 0}$ plane was appreciably reduced in the case of $\mathrm{CO}_{2}$ dilution. In addition, when plotted against computed flame temperature, it is clear that for SF flames (e.g., $1000 / T_{\mathrm{f}}[\mathrm{K}]>0.5$ ) at a fixed $X_{\mathrm{F}, 0}$, the flame temperatures at the sooting limit condition were higher in the $\mathrm{CO}_{2}$ atmosphere than those in $\mathrm{N}_{2}$. Furthermore, the temperature differences between the $\mathrm{CO}_{2}$ and $\mathrm{N}_{2}$ diluted flames were also higher for lower $X_{\mathrm{F}, 0}$ conditions (i.e., more dilution), providing further evidence of the chemical effects (in addition to temperature effects) of $\mathrm{CO}_{2}$ addition on soot inception. 

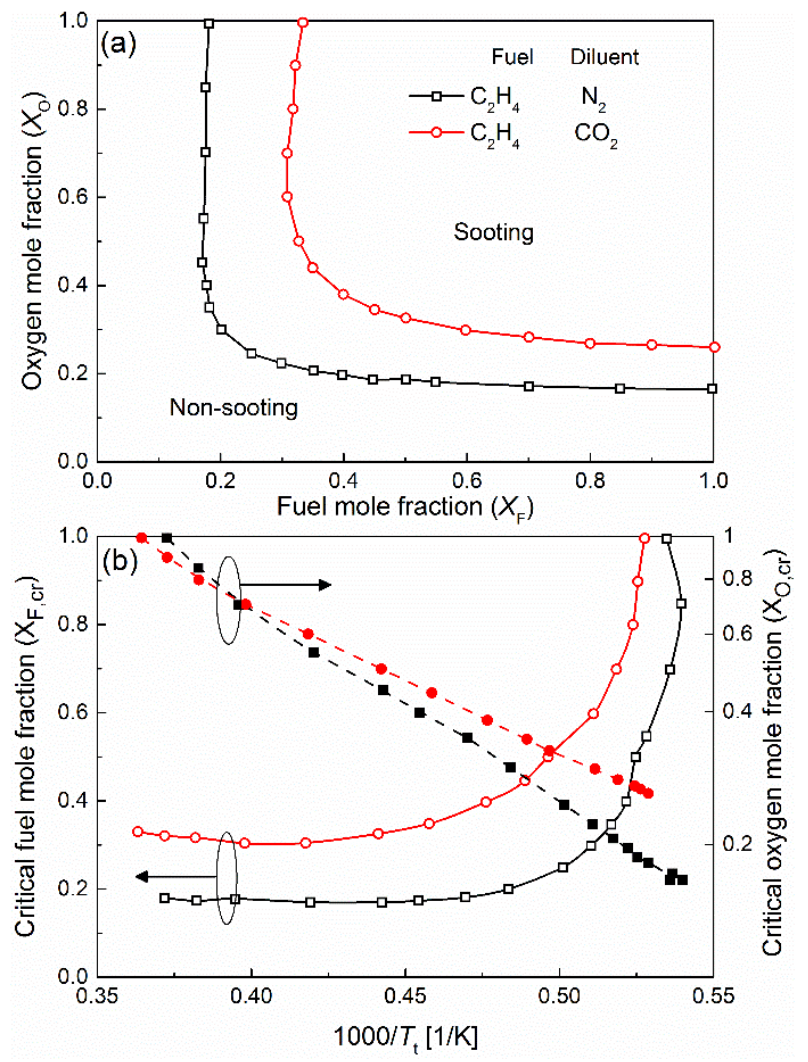

Figure 34. Sooting limit curves of $\mathrm{C}_{2} \mathrm{H}_{4}$ counterflow diffusion flames (a) and $X_{\mathrm{F}, \text { cr }}$ and $X_{\mathrm{O} \text {,cr }}$ as a function of calculated flame temperature (b) for $\mathrm{C}_{2} \mathrm{H}_{4}$ CDFs with $\mathrm{N}_{2}$ and $\mathrm{CO}_{2}$ dilutions. Data taken from Wang and Chung [626].

The above investigations in non-premixed flames consistently showed that $\mathrm{CO}_{2}$ addition can reduce soot formation, however, it is of interests to mention that there are also studies that indicated the opposite. For instance, Abian et al. [707, 711] observed that a $25 \% \mathrm{CO}_{2}$ addition in ethylene actually increased soot yield during experiments on temperature-controlled flow reactor fuel pyrolysis, although higher levels of $\mathrm{CO}_{2}$ addition led to reduced soot formation. The authors explained the enhancement of soot formation with a low level of $\mathrm{CO}_{2}$ addition, based on the increased $\mathrm{OH}$ radical concentrations through $\mathrm{CO}_{2}+\mathrm{H}=\mathrm{CO}+\mathrm{OH}$, which were expected to increase consumption of $\mathrm{H}_{2}$ by $\mathrm{OH}+\mathrm{H}_{2}=\mathrm{H}_{2} \mathrm{O}+\mathrm{H}$. The removal of $\mathrm{H}_{2}$ will favor $\mathrm{H}$ abstraction reactions in the HACA mechanism for PAH/soot growth and thus leads to higher soot production. At a higher dilution level, $\mathrm{CO}_{2}$ may result in a relatively large amount of $\mathrm{OH}$ radicals, which help to oxidize PAHs and soot particles. Similar enhancement of soot by $\mathrm{CO}_{2}$ was seen by Teini et al. [712] in 
experiments of $\mathrm{C}_{2} \mathrm{H}_{2}$ pyrolysis. Further kinetic simulation showed that the addition of $\mathrm{CO}_{2}$ enhanced the production of $\mathrm{C}_{3} \mathrm{H}_{3}$ (and thus benzene by $\mathrm{C}_{3} \mathrm{H}_{3}$ self-combination) by increasing $\mathrm{OH}$ radicals, which were also seen to help abstract $\mathrm{H}$ atoms from PAH molecules, allowing for further HACA growth. Note that in these pyrolysis experiments, the thermal effects of $\mathrm{CO}_{2}$ were not present because temperatures were held fixed in all cases, and the reactants are $\mathrm{O}_{2}$ deficient. In this way, these observations of $\mathrm{CO}_{2}$ increasing soot formation share many similarities with the finding that a small amount of $\mathrm{O}_{2}$ addition in the fuel side of $\mathrm{C}_{2} \mathrm{H}_{4}$ [165] and $\mathrm{C}_{2} \mathrm{H}_{2}$ [713] fuels actually enhanced soot formation. This is especially true considering the fact that $\mathrm{CO}_{2}$ addition was not found to enhance soot production during the pyrolysis of methane [712], consistent with Gülder's findings in methane coflow flames [714]. This will be discussed in more detail in a later section on partial premixing.

Compared to $\mathrm{CO}_{2}$, there are much fewer studies on the effects of water vapor addition on soot formation in CDFs, probably due to the additional experimental complications of metering and vaporizing liquid water. Equipped with a counterflow burner with the capability of reactant preheating, Zhang et al. [525] found that the addition of water vapor to both the oxidizer and fuel side could reduce soot formation, although addition on the oxidizer side was more effective. It was also qualitatively noticed that water vapor addition was more effective for soot inhibition than adding the same moles of $\mathrm{CO}_{2}$. Although no detailed kinetic simulation was performed, the authors reasoned that, with $\mathrm{H}_{2} \mathrm{O}+\mathrm{H}=\mathrm{OH}+\mathrm{H}_{2}$, the added water produced considerable amounts of $\mathrm{OH}$ radicals, which helped oxidize molecular soot precursors. The higher effectiveness of $\mathrm{H}_{2} \mathrm{O}$ compared to $\mathrm{CO}_{2}$ was explained simply by the fact that more $\mathrm{OH}$ radicals can be produced with $\mathrm{H}_{2} \mathrm{O}$ addition, as a consequence of the faster rate of $\mathrm{H}_{2} \mathrm{O}+\mathrm{H}=\mathrm{OH}+\mathrm{H}_{2}$ than $\mathrm{CO}_{2}+\mathrm{H}=\mathrm{OH}+\mathrm{CO}$ [715]. On the other hand, experimental results, as obtained with coflow diffusion flames, led Schug et al. [705] to conclude that $\mathrm{H}_{2} \mathrm{O}$ plays only a thermal effect. A similar conclusion was drawn by Rao and Bardon [716], who burned liquid hydrocarbon/water emulsions in diffusion flames. In an effort to clarify whether $\mathrm{H}_{2} \mathrm{O}$ can chemically affect soot formation, Liu et al. [717] conducted numerical simulations 
of sooting coflow diffusion flames of ethylene with oxidizer side $\mathrm{H}_{2} \mathrm{O}$ addition. Computations with fictitious $\mathrm{H}_{2} \mathrm{O}$ addition were included and it was observed that the chemical effects of $\mathrm{H}_{2} \mathrm{O}$ led to higher flame temperatures, but lower soot concentrations. Specifically, the addition of water reduced O radical concentration via $\mathrm{O}+\mathrm{H}_{2} \mathrm{O}=\mathrm{OH}+\mathrm{OH}$, so that the concentration of $\mathrm{CH}_{2}$, produced primarily from $\mathrm{C}_{2} \mathrm{H}_{2}+\mathrm{O}=\mathrm{CH}_{2}+\mathrm{CO}$, was also reduced. This explained the chemical effects of $\mathrm{H}_{2} \mathrm{O}$ in reducing the formation of aromatic soot precursors such as benzene and pyrene, because $\mathrm{CH}_{2}$ is essential in the formation of $\mathrm{C}_{3} \mathrm{H}_{3}$ (via e.g., $\mathrm{C}_{2} \mathrm{H}_{2}+\mathrm{CH}_{2}=\mathrm{C}_{3} \mathrm{H}_{3}+\mathrm{H}$ ), the self-recombination of which is the major benzene formation pathway. Furthermore, through $\mathrm{H}+\mathrm{H}_{2} \mathrm{O}=\mathrm{OH}+\mathrm{H}_{2}$, the concentration of $\mathrm{H}$ radical was also reduced, decreasing HACA growth rates.

\subsubsection{Partial-premixing}

Partial-premixing refers to the addition of oxygen — either pure or diluted by inert gases - in the fuel side of a non-premixed combustion system. The level of addition is typically kept low so that the equivalence ratio of the premixture is much larger than unity in the fuel stream. Motivation for studying the effect of partial premixing on soot formation includes the fact that a small amount of oxygen is frequently present in the fuel side of diffusion flames. As an example, for coflow flames which are widely adopted in studying soot formation, a reaction quenching occurs in regions in close proximity to the nozzle due to excessive heat and radical losses, so that a small amount of air entrainment is inevitable [109]. Oxygen penetration into the fuel side can also occur due to finite rate chemistry (i.e., incomplete combustion) [660]. From a practical point of view, air is sometimes intentionally co-injected with fuel in industrial combustion devices, to improve fuel atomization. It was also shown that in diesel engines ahead of the main diffusion flame zone a fuel-rich premixed region is always present influencing initial soot formation typically due to a lifted nature of flame base $[718,719]$. Furthermore, studies on partial-premixing may also clarify the underlying 
mechanisms that can help identify dominant soot/PAH growth pathways in flames of different fuels. Notable examples can be seen, for example, in the work of McEnally and Pfefferle [720].

A previous section presented the general sooting structures of partially-premixed CDFs; a more detailed and quantitative discussion on the hydrodynamic and chemical effects of partial-premixing on soot formation is given here. It is helpful to first show some direct images of partially premixed CDFs. Figure 35 presents the variation of the visual flames as an incipiently-sooting non-premixed counterflow flame (equivalence ratio $\phi=\infty$ ) transited towards partially-premixed flames up to $\phi=3$ (taken by Carbone et al. [81]). Note here that the stoichiometric mixture fraction $\left(Z_{\mathrm{st}}\right)$ and peak temperature were held constant as progressively more oxygen was added to the fuel stream, in order to maintain the location of the diffusion flame sheet and the thermal structure unchanged on the oxidizer side. It can be seen that-apart from the purely non-premixed flame-all the other partiallypremixed flames (PPF) featured double flame zones (represented by the $\mathrm{CH}$ chemiluminescence, in blue) with the bottom zone being rich premixed flame and the upper zone diffusion flame. The soot zones (yellow luminosity) were sandwiched between these two flames. With the aid of thermocouples for temperature measurement, and GC-MS for species quantification, Carbone et al. [81] performed careful experimental investigations to resolve the detailed chemical structures of these flames. It was shown that oxygen addition led to a much more rapid temperature rise as the fuel stream was convected downstream, as a result, there was more time for soot-related reactions to occur in the high temperature regions. In fact, increases in $\mathrm{C}_{3}, \mathrm{C}_{4}$ (except for $\mathrm{C}_{4} \mathrm{H}_{2}$ ), benzene, and soot concentrations were found as $\phi$ decreased. Further kinetic analysis also revealed that an enhanced contribution from $\mathrm{C}_{3}$ pathways to benzene formation was induced by oxygen addition. It should be mentioned that the lowest level of premixing tested by Carbone et al. [81] was already high enough $(\phi=6.5)$ to generate a separate rich-premixed flame. As seen later, there are other studies in which the level of premixing was much lower and no separate second flame can be observed. In these cases, the mechanism of 
partial premixing on soot formation can be different. In subsequent discussions, these cases will be termed as small amounts of oxygen addition.

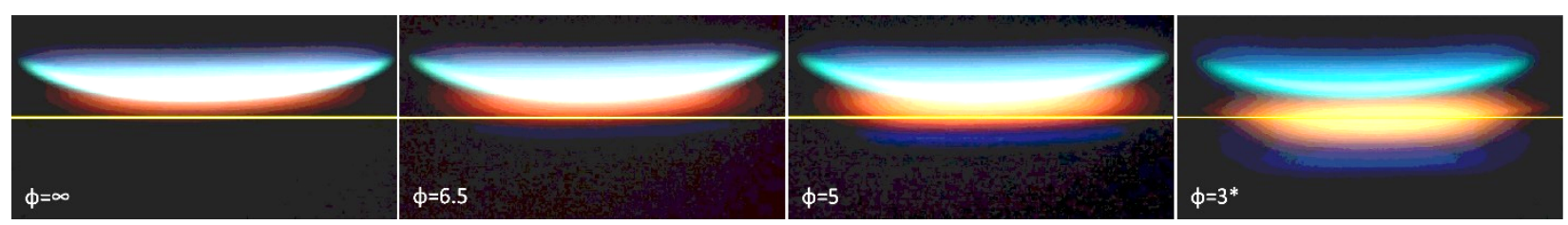

Figure 35. Direct images of partially-premixed ethylene counterflow flames with decreasing equivalence ratio in the fuel side, reprinted from Carbone et al. [81] with permission of Elsevier. Fuel and oxidizer streams issued from bottom and top, respectively. Yellow line indicates position of stagnation plane, and the flame with $\phi=3$ had a lower temperature than the rest.

Hura and Glassman [113] are among the pioneers to measure soot volume fraction in CDFs with partial premixing. A comprehensive investigation was performed to test the effects of small amounts of fuel side oxygen addition on soot formation in CDFs of ethylene, propene, propane, n-butane, and iso-butane [113]. The results showed that soot volume fraction, average particle sizes, and surface growth rates were all noticeably increased with an addition of $10 \%$ oxygen to the ethylene flame (a doubling of peak SVF); while the enhancing effects were rather small for propene flame, with even suppressive effect observed in propane and iso-butane flames. The authors noticed that oxygen addition in ethylene led to an increased amount of $\mathrm{H}$ radical, which helped to promote fuel pyrolysis rate. However, no details were reported regarding important soot precursors. Realizing that fuel side oxygen addition may increase flame temperature, and thus thermally affect soot formation, Hura and Glassman, in a subsequent study [721], manipulated the oxidizer composition of the CDF to keep the peak flame temperature unchanged. They also extended the range of oxygen addition from an equivalence ratio of infinity (purely diffusion flame) to 3.0 and 2.6 for ethylene and propane flames, respectively. Major results of this study are re-plotted in Figure 36 in terms of the variation of peak extinction coefficient (i.e., an indication of SVF) with equivalence ratio. Similar soot enhancing/inhibiting effects were observed for small amounts of oxygen addition (large equivalence 
ratio, to avoid significant variations in flame structures) in ethylene (propane) flames. With further addition of oxygen, both flames exhibited a sharp increase, followed by a rapid decrease in soot formation, attributable to the drastic change of flame structures. While enhanced fuel pyrolysis rates explained the initial soot increase in ethylene, fuel dilution was believed to be the dominant factor in decreasing soot production in propane CDF.

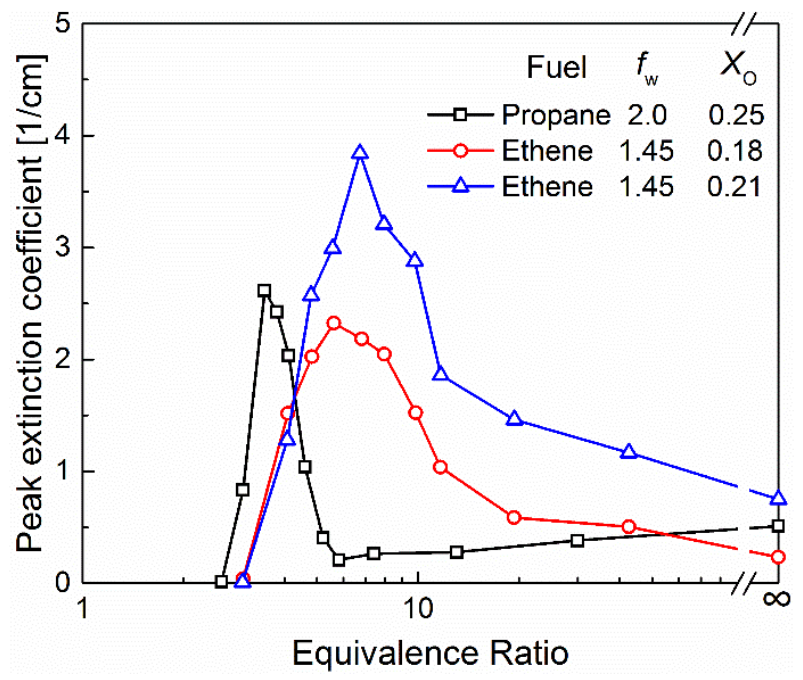

Figure 36. Peak extinction coefficients for ethylene and propane partially premixed counterflow diffusion flames as a function of fuel stream equivalence ratio, $f_{\mathrm{w}}$ is fuel flow rate. Reprinted from Hura and Glassman [721] with permission of Elsevier.

The fact that small amounts of oxygen chemically enhance ethylene sooting tendency was confirmed by a number of other investigations in CDFs $[165,564]$ and coflow diffusion flames [564, $705,714,722-727]$. The influence of oxygen in propane flames, on the contrary, has been the subject of much debate, and disagreement appeared at two levels. First, contradictory results were reported in different studies regarding the overall effect of fuel side oxygen addition on soot formation. For instance, Du et al. [564] showed that the critical strain rate $K_{\mathrm{cr}}$ (i.e., sooting tendency) of propane CDF increased monotonically with the level of oxygen addition, up to $50 \%$, while Hura and Glassman [113] observed a decrease in peak SVF when 10\% oxygen was added. Similarly, in coflow diffusion flames of propane, increased soot productions were seen in [564, 725, 728], but reduced soot formation was observed in $[721,722,724]$ with a small amount of oxygen addition. No definite 
explanation was found to explain these discrepancies. The second level of disagreement concerned the chemical effects of oxygen addition. Even though the phenomenon of soot reduction observed by Hura and Glassman [721] was qualitatively consistent with the experimental results of Gülder [714], explanations for the reduction differed. Specifically, through reactant preheating to maintain adiabatic flame temperature and comparison against the $\mathrm{N}_{2}$-diluted flames, Gülder experimentally separated the chemical effects of oxygen addition from thermal and dilution effects. His results showed that oxygen significantly enhanced soot formation through its chemical interactions [714]. However, Hura and Glassman [721] concluded that oxygen was mainly a fuel diluent, with insignificant chemical effects, and Du et al. [564] showed a significantly suppressive role for oxygen in propane flame. Note that although the work of Hwang and Chung [165] showed a reduction of SVF in propane CDFs with oxygen addition, in their study the oxidizer that comprised both oxygen and nitrogen was added to the fuel stream (to maintain temperature), so that the observed soot reduction was a combined effect of chemical and dilution, and it was unclear whether or not soot formation would be suppressed if allowance was made for the dilution effects. It appears that additional experiments-especially those accompanied by detailed kinetic simulation-are required to clarify this matter.

Based on a detailed gas-phase kinetic simulation [165], the enhancement of soot formation by oxygen addition in ethylene CDFs was explained through the increased reaction rates of $\mathrm{C}_{2} \mathrm{H}_{2}+\mathrm{O}=$ $\mathrm{CH}_{2}+\mathrm{CO}$. Methylene $\left(\mathrm{CH}_{2}\right)$ is essential for the formation of $\mathrm{C}_{3} \mathrm{H}_{3}$ - thus the first aromatic ring. With on-line mass spectrometry measurements, McEnally and Pfefferle [68] experimentally observed (and later confirmed by numerical simulations $[729,730])$ that peak concentrations of $\mathrm{CH}_{4}, \mathrm{C}_{3} \mathrm{H}_{3}$, and 1,3$\mathrm{C}_{4} \mathrm{H}_{6}$ increased with the addition of oxygen (in the form of air) in the fuel stream of ethylene coflow diffusion flames, providing support for the $\mathrm{C}_{3} \mathrm{H}_{3}$-based explanation in [165]. (Note that 1,3- $\mathrm{C}_{4} \mathrm{H}_{6}$ can be formed with the addition of $\mathrm{CH}_{3}$ to $\mathrm{C}_{3} \mathrm{H}_{3}$.) In addition to the above-mentioned reaction for methylene production, it was also shown that oxygen addition can enhance $\mathrm{C}_{2} \mathrm{H}_{4}+\mathrm{O}=\mathrm{CH}_{3}+\mathrm{HCO}$ to produce $\mathrm{CH}_{3}$, which is then involved in various $\mathrm{C}_{1}+\mathrm{C}_{2}$ interactions to enhance $\mathrm{C}_{3}$ species 
formation. As mentioned in previous discussions regarding the synergistic effects of $\mathrm{C}_{2} \mathrm{H}_{4}$-based binary fuel mixtures, $\mathrm{C}_{2} \mathrm{H}_{4}$ flames are particularly sensitive to increased $\mathrm{C}_{1}$ species from either fuel dopants or oxidative pyrolysis, because it is somewhat difficult to break the strong $\mathrm{C}=\mathrm{C}$ double bond at pyrolysis temperatures [713], which leads to a deficiency of the $C_{1}$ species. Following this reasoning, similar phenomenon can be expected in $\mathrm{C}_{2} \mathrm{H}_{2}$ diffusion flames, considering the strength of the $\mathrm{C} \equiv \mathrm{C}$ triple bond. In fact, in the $\mathrm{C}_{2} \mathrm{H}_{2}$ CDFs studied by Leusden and Peters [75], small amounts of added oxygen were seen to chemically enhance the formation of $\mathrm{C}_{3} \mathrm{H}_{4}$, benzene, and soot. Further kinetic analysis showed that both the $\mathrm{C}_{3}$ and $\mathrm{C}_{4}+\mathrm{C}_{2}$ channels for benzene formation were promoted. Similar results were also noticed in coflow diffusion flames of $\mathrm{C}_{2} \mathrm{H}_{2}$ [713]. Since 1,3-butadiene $\left(\mathrm{CH}_{2}=\mathrm{CH}-\mathrm{CH}=\mathrm{CH}_{2}\right)$ shares a feature with $\mathrm{C}_{2} \mathrm{H}_{4} / \mathrm{C}_{2} \mathrm{H}_{2}$ in that its pyrolysis does not easily produce $\mathrm{C}_{1}$ species, it may be expected that oxygen addition would also increase soot formation in 1,3-butadiene flames. However, experimental data in coflow diffusion flames showed the opposite to be true. It was postulated by McEnally and Pfefferle [713] that 1,3-butadiene may isomerize to $\mathrm{CH}_{2}=\mathrm{C}=\mathrm{CH}-\mathrm{CH}_{3}$ so that $\mathrm{C}_{1}$ and $\mathrm{C}_{3}$ species can be easily produced by $\mathrm{C}-\mathrm{C}$ bond breaking. Alternatively, the dominant benzene formation channels for 1,3-butadiene may be through even carbon chemistry (making $\mathrm{C}_{3}$ chemistry less important), which has been partially confirmed by a recent chemical speciation study [174].

Partial-premixing is also interesting for methane flames, since natural gas appliances typically rely on partially-premixed flames. Mungekar and Atreya [561, 562] studied the effects of partialpremixing on soot formation in low strain rate methane CDFs, for both the SF and SFO configurations. For SF flames, the authors observed that with increasingly higher levels of oxygen addition, the soot zone thickness first decreased (from $\phi=\infty$ to 8.3 ) and then increased (from $\phi=8.3$ to 6.4). While the initial decrease (consistent with the observations of Nakamura et al. [731] in a methane CDF with $\phi$ decreasing from infinity to 10) was thought to be caused by the shrinkage of the distance between the non-premixed flame front and the stagnation plane, it was presumed that the subsequent increase was 
due to the presence of a separate high-temperature rich premixed flame front, on the fuel side of the non-premixed flame front, where soot began to form. Peak SVFs were observed to decrease as $\phi$ decreased from infinity to 8.3 ; this was explained based on the dilution and chemical suppressive role of $\mathrm{O}_{2}$, which was also shown to contribute to the soot reduction in methane coflow diffusion flames with partial-premixing [714, 732]. As $\phi$ further decreased, the high temperature non-premixed flame front moved closer to the stagnation plane, leading to increased temperature and longer soot residence time in high temperature regions, and resulting in an increase in SVF. In SFO flames, where the flame is located on the fuel side of the stagnation plane, fuel stream partial premixing was seen to monotonically decrease soot formation; while it was noted that such a decrease was consistent with the competition between soot inception, growth and oxidation, no detailed explanations were provided.

Although without soot measurements, Berta et al. performed temperature and intermediate species measurements for pre-vaporized n-heptane non-premixed flames [592] and PPFs [563, 584] in a counterflow configuration, with special focus on gaseous soot precursors, including $\mathrm{C}_{2} \mathrm{H}_{2}$ and benzene. The oxidizer streams in their flames were air in all cases, while the fuel stream consisted of n-heptane vapor diluted with various amounts of air, producing various equivalence ratios. The structures of the PPFs were resolved both experimentally and numerically, with satisfactory agreements. The axial profiles of temperature, velocity, $\mathrm{C}_{2} \mathrm{H}_{2}, \mathrm{C}_{2} \mathrm{H}_{4}$ and $\mathrm{C}_{6} \mathrm{H}_{6}$ are shown in Figure 37 for various equivalence ratios. Results showed that as $\phi$ decreased, the diffusion flame front tended to move closer to the fuel nozzle, consistent with the work of Mungekar and Atreya [561, 562], and much as expected, considering the variations in stoichiometric requirements. The rich premixed flame zone (indicated by the bump in the velocity profile on the fuel side of the diffusion flame) was also seen to move to the fuel nozzle as $\phi$ decreased, which can be rationalized by considering the balance between the stretched laminar flame speed and the incoming reactant velocity. As $\phi$ decreased from infinity, the flame speed of the pre-mixture increased so that the flame front moved to a location at a 
higher reactant speed, i.e., closer to the fuel nozzle. The stagnation plane, on the contrary, moved away from the fuel nozzle as $\phi$ decreased. An increased level of partial-premixing was seen to monotonically reduce $\mathrm{C}_{2} \mathrm{H}_{2}, \mathrm{C}_{2} \mathrm{H}_{4} \mathrm{C}_{6} \mathrm{H}_{6}$, as well as coronene $\left(\mathrm{C}_{24} \mathrm{H}_{12}\right)$, and the effects were much stronger for large PAHs (mole fractions for coronene, $\mathrm{C}_{6} \mathrm{H}_{6}$ and $\mathrm{C}_{2} \mathrm{H}_{2}$ decreased by a factor of around $1,000,10$ and 2, respectively, as $\phi$ decreased from 15 to 2.5 [563]). This was attributed by the authors to reduced residence time and enhanced oxidation. This result contrasts with the observations of Mungekar and Atreya [561] in methane PPFs, where an increase of soot was observed as $\phi$ decreased from 8.3 to 6.4. The major difference was that while Mungekar and Atreya [561] partially-premixed the fuel with pure oxygen, Berta et al. [563] added air in the fuel stream so that a large amount of $\mathrm{N}_{2}$ was present in the fuel stream with partial-premixing. In these cases, significant fuel dilution may have been the dominant contributor to the observed soot reduction.
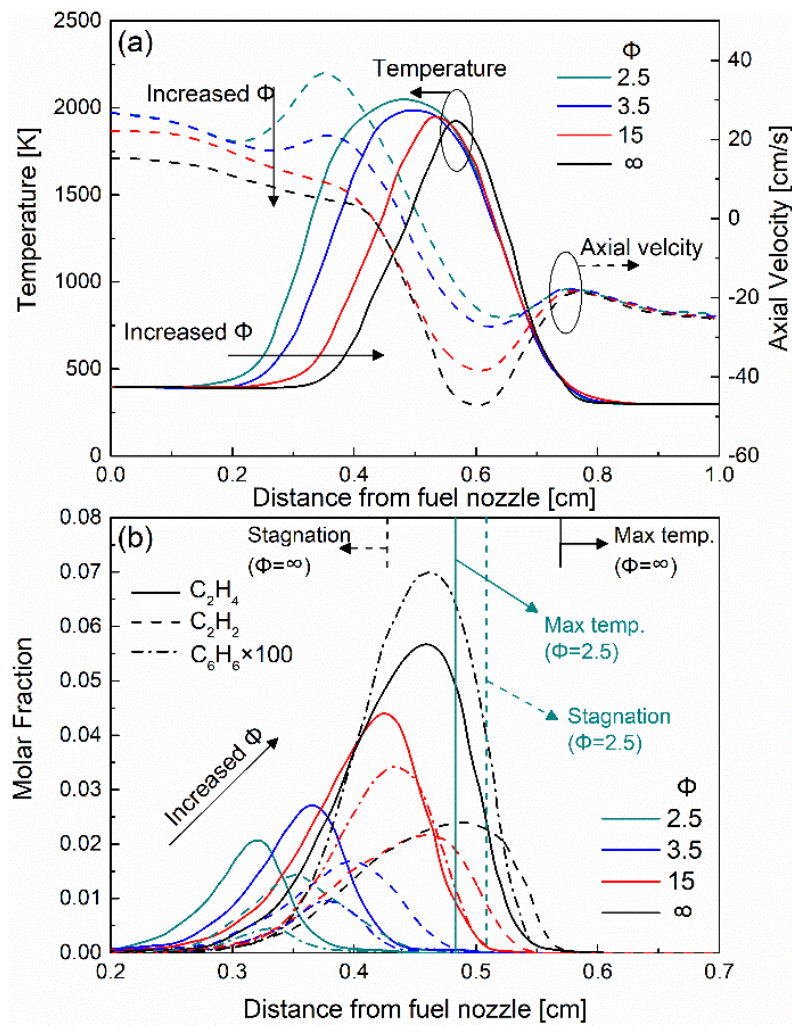

Figure 37. Axial profiles of temperature and axial velocity (a), and $\mathrm{C}_{2} \mathrm{H}_{2}, \mathrm{C}_{2} \mathrm{H}_{4}$ and $\mathrm{C}_{6} \mathrm{H}_{6}$ mole fractions (b) of partially-premixed counterflow diffusion flames at various equivalence ratios. Data taken from Berta et al. [563]. 


\subsubsection{Other dopants}

\section{a) addition of chlorinated species}

Interest in halogenated species originated with their use as flame extinguishing agents [733-735], primarily due to their ability to scavenge the highly reactive $\mathrm{H}$ radicals in hydrocarbon flames [736]. There were also concerns about environmental pollution and the problems of incineration-based disposal of hazardous wastes [737], of which chlorinated species (as in chemical solvents, polychlorinated biphenyl (PCB) and polyvinyl chloride) may account for a considerable fraction.

Many investigations in shock tubes [738] and premixed flames [739, 740], as well as diffusion flames [741], have shown that chlorinated compounds can enhance PAH/soot formation, as compared to their hydrocarbon counterparts. For instance, a systematic work of Senkan et al. [739] showed that the critical equivalence ratio $\left(\phi_{\mathrm{cr}}\right)$ for soot formation decreased in a methane-based premixed flame with the addition of $\mathrm{CH}_{2} \mathrm{Cl}_{2}, \mathrm{CHCl}_{3}$, and $\mathrm{CCl}_{4}$, and the effects were stronger for molecules containing more chlorine atoms. A critical review of relevant studies until the early 1990s was provided by Morse and Cundy [742]. Subsequent works included the development of detailed kinetic models to understand fundamental combustion characteristics of chlorinated hydrocarbons (CHC) [420, 743745]. Several possible reasons were provided to explain the higher sooting tendencies of CHCs: First, as the $\mathrm{C}-\mathrm{Cl}$ bond is noticeably weaker than $\mathrm{C}-\mathrm{H}$ bond [746], initial fuel pyrolysis is easier for $\mathrm{CHCs}$, which can lead to higher concentrations of the radicals essential for PAH/soot growth $[737,738,740]$; Second, the chlorine atom enhances $\mathrm{H}$-abstraction reaction from stable $\mathrm{PAH}$ molecules through $\mathrm{A}_{i}+$ $\mathrm{Cl} \rightleftharpoons \mathrm{A}_{i^{-}}+\mathrm{HCl}$, leading to more aromatic radicals for further HACA growth [737]; Third, chlorine may also scavenge oxidative radicals like $\mathrm{O}$ and $\mathrm{OH}$, resulting in reduced soot oxidation [740], although such effects are not present in oxygen-free pyrolysis experiments.

Relevant studies conducted in CDFs are somewhat scarce. Yang and Kennedy [569] performed an experimental investigation of CDFs fueled with pure $\mathrm{CH}_{4}$ and $\mathrm{CH}_{4} / \mathrm{CH}_{3} \mathrm{Cl}$ binary mixtures, with air 
as the oxidizer, and at a constant local strain rate $K_{\mathrm{L}}$ of $38.7 \mathrm{~s}^{-1}$. The neat $\mathrm{CH}_{4}$ flames did not produce measurable amounts of soot, as a result, only the soot volume fraction profiles of the more sooting $\mathrm{CH}_{3} \mathrm{Cl}$-doped flames were measured. The data showed that the sooting structure of $\mathrm{CH}_{3} \mathrm{Cl}$-doped flame was qualitatively similar to a typical hydrocarbon SF CDF. However, quantitatively, peak SVF increased monotonically-and almost linearly-with the molar ratio of $\mathrm{CH}_{3} \mathrm{Cl}$ in the $\mathrm{CH}_{4} / \mathrm{CH}_{3} \mathrm{Cl}$ mixtures. Leylegian [594] measured the critical sooting strain rate $\left(K_{\mathrm{cr}}\right)$ in $\mathrm{CDFs}$ of $\mathrm{CH}_{4} / \mathrm{CH}_{3} \mathrm{Cl}$ and $\mathrm{C}_{2} \mathrm{H}_{4} / \mathrm{CH}_{3} \mathrm{Cl}$ binary mixtures and found a nearly linear increase of $K_{\mathrm{cr}}$ with a molar mixing ratio of $\mathrm{CH}_{3} \mathrm{Cl}$, a result that was qualitatively consistent with that of Yang and Kennedy [569]. An interesting phenomenon was observed for $\mathrm{C}_{2} \mathrm{H}_{4} / \mathrm{CH}_{3} \mathrm{Cl}$ mixture in that $K_{\mathrm{cr}}$ first increased and then decreased, exhibiting a non-monotonic variation trend with $\mathrm{CH}_{3} \mathrm{Cl}$ mixing ratio. The author believed this was caused by chemical interactions between the mixture components, i.e., the initial increase can be attributed to the effect of chlorine on the fuel pyrolysis rates, while the latter decrease was due to the lowered ethylene concentration. However, it is important to point out that in order for the author to maintain the carbon density (in $\mathrm{g} / \mathrm{cm}^{3}$ ) constant in the fuel stream, the $\mathrm{N}_{2}$ dilution (mole fraction) was higher in cases with lower $\mathrm{CH}_{3} \mathrm{Cl}$ mixing ratio, so that the different dilution levels may also play a role.

\section{b) addition of metal-containing compounds}

Investigations on combustion of hydrocarbon fuels doped with metal-containing compounds are generally related to the following topics: 1) flame inhibition [747-749]; 2) synthesis of metal oxide nanoparticles [750-752] and desired carbon nanostructures (i.e., carbon nanotubes) [753-755]; 3) $\mathrm{PM} /$ soot emission reduction [118, 756-759]. Here we are concerned only with soot, and it was found that iron-containing compounds such as ferrocene and iron-pentacarbonyl are studied most.

Several mechanisms have been proposed to explain the effects of metal additives on soot formation: First, some metals, such as sodium and potassium, can easily become ionized in flame environments. 
Their charges can subsequently be transferred to soot precursors so that the rates of soot nucleation are effectively reduced [118]. Although more evidence is needed, this mechanism is known to be consistent with observations of rich premixed ethylene flames where potassium chloride $(\mathrm{KCl})$ doping decreased both the soot volume fraction and the particle size [756, 760]; Second, metal containing compounds can scavenge the active radicals of $\mathrm{H}, \mathrm{O}$ and $\mathrm{OH}$, affecting the fuel pyrolysis processes [748], through which $\mathrm{PAH} /$ soot formation can, in turn, be influenced; Third, metalcontaining compounds-especially iron-containing species-can decompose to form metal nanoparticles which serve as the nuclei to provide additional surface sites for soot growth [761]; Fourth, metal-containing species, when incorporated in soot particles, can notably catalyze soot oxidation [263]. Note that the third and fourth mechanisms contribute in opposite ways to the final soot emission, while both are concretely supported by data in the literature. For instance, shock tube studies showed that soot yields for $\mathrm{C}_{3} \mathrm{H}_{8} / \mathrm{Ar}$ and $\mathrm{C}_{2} \mathrm{H}_{2} / \mathrm{Ar}$ mixtures can be significantly enhanced after the addition of a small amount of iron pentacarbonyl $\left(\mathrm{Fe}(\mathrm{CO})_{5}\right)$ [762]. Studies in rich premixed flames, where a deficiency of oxidizing species limits soot oxidation, have shown enhanced soot formation after doping of either ferrocene or $\mathrm{Fe}(\mathrm{CO})_{5}[761,763,764]$. It was also observed that in diffusion flames, particles appeared earlier in iron-seeded flames than in unseeded flames [262, 263, 634, 765]. These studies supported the third mechanism, as discussed above [757]. Strong evidence also exists for the fourth mechanism, both for in-flame measurements near the oxidizing flame front [263, 757], and ex-situ oxidation measurements of iron-containing soot [766]. In particular, the oxidation activation energy for soot formed in iron-containing environments was found to be $116 \mathrm{~kJ} / \mathrm{mol}$, significantly lower than the $162 \mathrm{~kJ} / \mathrm{mol}$ for soot formed in unseeded flames [767]. Furthermore, a recent study suggested that the iron-containing particles that catalyze soot oxidation were primarily elemental $\mathrm{Fe}$, rather than any form of iron oxide [768]. It is generally accepted that soot reduction by metal addition (observed in many practical combustion devices) is a result of more efficient burnout, rather than inhibition of soot nucleation [761]. 
Relevant studies in CDFs were performed by Raj et al. $[261,634,769]$ who doped $\mathrm{Fe}(\mathrm{CO})_{5}$ in a methane CDF with $X_{\mathrm{F}, 0}=0.097, X_{\mathrm{O}, 0}=0.53$ and an effective strain rate $K_{\mathrm{E}}$ of $17 \mathrm{~s}^{-1}$. Of special note is that $\mathrm{Fe}(\mathrm{CO})_{5}$ was carried by a separate $\mathrm{N}_{2}$ stream and fed to the flame through a central tube embedded in — and concentric with—-the bottom fuel burner nozzle. As a result, the flame was not quasi-1D, as in conventional CDFs. The authors observed a significant decrease in the concentrations of $\mathrm{C}_{2}$ species (i.e., $\mathrm{C}_{2} \mathrm{H}_{6}, \mathrm{C}_{2} \mathrm{H}_{4}$ and $\mathrm{C}_{2} \mathrm{H}_{2}$ ) with $\mathrm{Fe}(\mathrm{CO})_{5}$ addition and attributed these reductions to the catalytic combustion of methane by iron oxide [261]. This explanation was consistent with the observed reduction of $\mathrm{CO}$ and faster consumption of $\mathrm{CH}_{4}$, while it seemed incompatible with the data of Kim and Hahn [768], which indicated the dominance of elemental iron over iron oxide of the diffusion flames. Nevertheless, in a later modelling work of these flames, Raj et al. [769] mentioned that the reduction of $\mathrm{C}_{2}$ species could be due to scavenging of $\mathrm{O}, \mathrm{H}$ and $\mathrm{OH}$ radicals by iron species; while these radicals could have contributed to molecular growth from methane to $\mathrm{C}_{2}$ species. Perhaps the most striking observation of Raj et al. was the presence of large amounts of $\mathrm{O}_{2}$ in the fuel side for the seeded methane CDF, even at locations very close to the fuel outlet [261]. The authors explained this interesting behavior by the diffusion of oxygen across the stagnation plane, due to early consumption of the fuel; unfortunately, however, no oxygen concentration profiles were provided in a subsequent modelling study [769] for further confirmation. Raj et al. [634] continued to study the particle evolution of the same $\mathrm{CDF}$ with and without $\mathrm{Fe}(\mathrm{CO})_{5}$ addition. With tube sampling of the flame gas and subsequent particle analysis with a SMPS, the total particle number density and the size distributions were measured at different axial positions between the two opposing nozzles. Major results are shown in Figure 38, where it can be seen that under catalytic conditions, particles appeared earlier near the fuel side, and the peak soot number density was higher than in the unseeded case. However, the peak average particle diameter was smaller for the seeded flame, suggesting that the addition of $\mathrm{Fe}(\mathrm{CO})_{5}$ resulted in the formation of larger numbers of smaller particles. The reduced $\mathrm{C}_{2} \mathrm{H}_{2}$ concentration [261] (thus slower HACA growth), and possibly reduced coagulation rates in the 
seeded flame, may help rationalize such findings. More interesting is that both the soot number density and average size in the seeded flame were seen to decrease more rapidly towards the flame front, offering evidence for the catalytic effect of iron-containing species on soot oxidation, and consistent with previous studies [261].

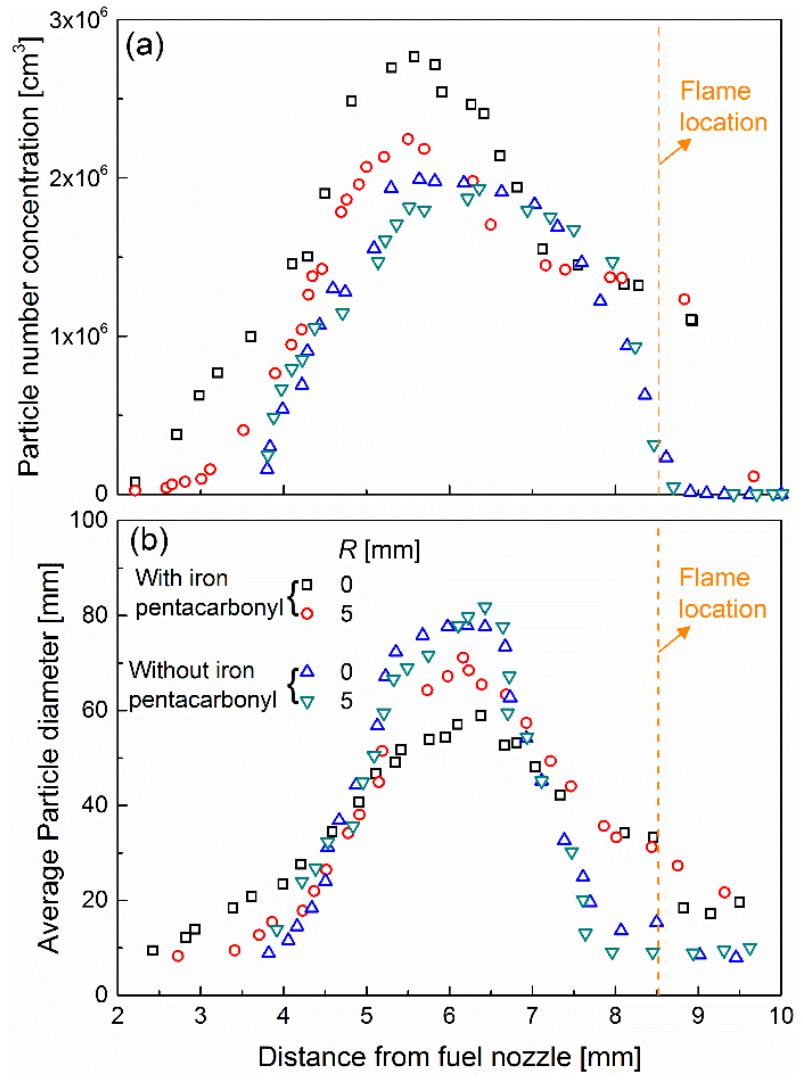

Figure 38. Particle number concentrations (a) and average particle diameters (b) of CDFs with and without fuel-side iron pentacarbonyl addition. Note the axial profiles along both the axis of symmetry $(\mathrm{R}=0 \mathrm{~mm})$ and along a radially shifted vertical line $(\mathrm{R}=5 \mathrm{~mm})$ are shown, considering the influence of the central tube for precursors. Adapted from Raj et al. [634] with permission of Elsevier.

It is noted that there are other dopants, such as sulfur- and nitrogen-containing compounds (i.e., $\mathrm{H}_{2} \mathrm{~S}, \mathrm{SO}_{2}, \mathrm{SO}_{3}, \mathrm{NH}_{3}, \mathrm{NO}$ and $\mathrm{NO}_{2}$ ) which have been tested for their effects on soot formation [119]; however, since no relevant studies were performed in CDFs, these dopants will not be discussed further here. 


\subsection{Effects of pressure on soot formation}

Energy conversion systems such as IC engines, jet engines and gas turbines typically rely on combustion at high pressure for maximum thermal efficiency and energy-releasing intensity, making it of practical interests to study soot formation at high*pressure conditions. Understanding the dependence of soot formation kinetics on pressure is also of vital importance from a scientific point of view. Experimental investigations of soot formation in high-pressure flames are notably more difficult than those performed at atmospheric conditions, partly because of flame stability issues [107] and partially due to the additional complexities of apparatus and instrumentation [127]. Also, the reduced flame thickness and excessive soot formation at high-pressure conditions can cause significant difficulties for quantitative soot measurements. Nevertheless, many researchers have successfully established steady flat premixed flames [770] and coflow diffusion flames [20] at pressures up to $100 \mathrm{~atm}$.

A review of early studies on pressure effects was provided by McArragher and Tan [771]. Subsequently, optical methods such as color pyrometry and laser light extinction / scattering were frequently applied in investigations of sooting characteristics in high-pressure flames. For premixed flames, focus was on the variations of final SVF, soot growth rate, particle number density and concentrations of important gaseous intermediates with pressure [770, 772-777]. In particular, at a fixed equivalence ratio and temperature, the final SVF were seen to increase with pressure $(P)$ proportional to $P^{2}$ up to several bar (approximately 7 bar) [772], beyond which a linear relationship between SVF and pressure was observed, indicating the absence of further soot mass growth at those extremely high-pressure conditions [770]. Later numerical calculations confirmed the observed pressure dependence [778] and the decay of soot growth was found to be caused either by decreasing particle activity at moderate pressure (15 bar) [774], or the lack of a growth agent (i.e., $\mathrm{C}_{2} \mathrm{H}_{2}$ ), at even higher pressures (70 bar) [770]. Similarly, the effect of pressure on the radical levels, especially those of $\mathrm{H}$ radicals, which plays an important role in $\mathrm{PAH}$ and soot growth, can be notable. It was also 
observed that pressure increase tended to reduce particle coagulation rates, attributed to the alteration of soot surface properties in high-pressure conditions that affect particle-particle sticking [776].

Soot formation in high-pressure diffusion flames has received more attention in the last decade, justifiable by the dominance of non-premixed combustion in soot-emitting practical combustion systems. A comprehensive review of soot studies in laminar diffusion flames before 2012 was provided by Karatas and Gülder [107], in which coflow flames has been investigated to the greatest extent. The advantage of coflow diffusion flames with concentric circular nozzles is that the flame heights can be maintained reasonably independent of pressure, provided that the fuel mass flow rate (i.e., in $\mathrm{mg} / \mathrm{s}$ ) is fixed $[107,779,780]$. Also, at a given height above the fuel outlet, the average velocity does not vary much with pressure because the increase in flow density is balanced by the decrease in the flow cross-section area $[483,781]$. As a result, soot volume fraction can be directly compared at the same height to flames under different pressure conditions, providing tractability of the pressure effects. From the review by Karatas and Gülder [107], it is noted that before 2012, flames of gaseous fuels accounted for the majority of these studies. At a constant fuel mass flow rate, increases in pressure generally led to a narrowing of the flame, resulting in increasingly curved inwards. Peak SVF, line-integrated SVF, and maximum conversion of fuel carbon to soot, all increased with pressure in the form [soot] $\propto P^{n}$, where $n$ was fuel dependent [61] and became smaller in the higher end of the pressure ranges [483]. The increase of soot yield resulted not only from flame narrowing, but from overall soot formation enhancement. Computational studies $[782,783]$ suggested that the pressure increase results in earlier soot inception, but later oxidation, thus experiencing more growth time. It was also shown [784] that high pressure forces coflowing air to infiltrate into the fuel side near the flame base, resulting in enhanced fuel pyrolysis.

More recently, research interest has gradually shifted towards pre-vaporized liquid fuels such as ethanol [785], $\mathrm{C}_{6}$ hydrocarbon and oxygenated species [21], n-heptane $[486,786,787]$ and n-decane [445]. The effects of pressure on soot properties other than SVF were also tackled, using light 
scattering [69] and/or thermophoretic sampling [509]. This section is intended to complement the review on coflow high-pressure flames in 2012 [107] by examining soot formation in high-pressure CDFs. In fact, Figura and Gomez [788] showed that CDFs are largely immune to buoyancy-driven instabilities, while this may pose significant issues for high-pressure coflow diffusion flames, especially those with elongated flame shapes, and thus, higher Richardson numbers. Together with its advantages of quasi-1D structures, clean boundaries and easy control of residence time, CDF is believed to be an ideal flame reactor for soot studies in high-pressure non-premixed systems. Nevertheless, upper constraints in the pressure range for steady CDF are caused by the transition towards turbulent flames, although the use of helium as a diluent may delay such transition [788].

The sooting limits represented by critical strain rate $K_{\text {cr }}$ were experimentally determined by Du $e t$ al. [573] for ethylene-air CDFs in the pressure range from 1 to $2.5 \mathrm{~atm}$; the results are summarized in Figure 39. Similar to previous studies at atmospheric pressure [551], laser light scattering was used to identify critical sooting conditions; and partial substitution of $\mathrm{N}_{2}$ with $\mathrm{Ar}$ in the oxidizer stream helped maintain the peak flame temperature as the pressure was varied. As can be seen, $K_{\mathrm{cr}}$ increased monotonically, indicating an enhanced sooting tendency as pressure increased (a). Also as expected, at a specified pressure, $K_{\mathrm{cr}}$ decreased with the decrease of $X_{\mathrm{F}, 0}$, demonstrating the effects of dilution on soot formation. The authors argued that the effects of pressure would be better represented by density-weighted critical strain rate, considering that, instead of the purely kinematic strain rate $K$, the characteristic reaction rates in CDF are proportional to the density-weighted strain rate $\rho_{0} K[789]$ (where $\rho_{0}$ is the density of the oxidizer at the nozzle exit). Interestingly, a linear relationship was observed between $\rho_{0} K_{\mathrm{cr}}$ and pressure $P(\mathrm{~b})$, and it was further shown that the data collected at different $X_{\mathrm{F}, 0}$ collapsed into a single line in the form of $\rho_{0} K_{c r} \sim X_{F, 0}^{1 / 2} P(\mathrm{c})$. Accompanying numerical simulation showed that the maximum partial pressure of $\mathrm{C}_{2} \mathrm{H}_{2}$ was also linearly correlated with $X_{F, 0}^{1 / 2} P$, so that $\rho_{0} K_{c r} \sim P_{\mathrm{C} 2 \mathrm{H} 2}$. With these results, the authors concluded that the soot formation rate in the investigated $\mathrm{C}_{2} \mathrm{H}_{4}$ flames was of first order with $\mathrm{C}_{2} \mathrm{H}_{2}$ concentration [573]. In a follow-up study [85], $\mathrm{CH}_{4}$ and 
$\mathrm{C}_{3} \mathrm{H}_{8}-$ air CDFs were tested with an extended pressure ranging from 1 to $5 \mathrm{~atm}$. The correlation

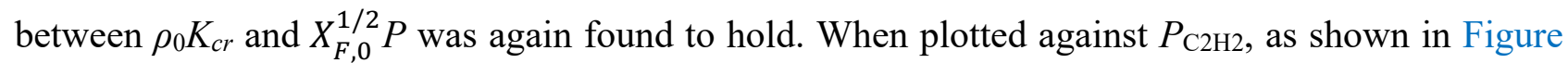
40 , the $\rho_{0} K_{c r}$ data for $\mathrm{C}_{3} \mathrm{H}_{8}$ and $\mathrm{C}_{2} \mathrm{H}_{4}$ flames collapsed into a single straight line, while the data for the $\mathrm{CH}_{4}$ flame followed a distinctively different line. The authors rationalized the similarity between $\mathrm{C}_{3} \mathrm{H}_{8}$ and $\mathrm{C}_{2} \mathrm{H}_{4}$ flames by pointing out that $\mathrm{C}_{2} \mathrm{H}_{4}$ was among the primary products of fuel-side propane pyrolysis. Sarnacki and Chelliah [530] performed absolute irradiance-calibrated time-resolved LII and PIV measurements to quantify SVF and flow fields, respectively, in ethylene CDFs up to 30 atm. While direct comparisons of soot loadings in flames of different pressures were difficult because different dilution/strain rates were used as pressure varied, the authors showed that global activation energy for nucleation increased exponentially with pressure up to $20 \mathrm{~atm}$, beyond which a sudden decrease was observed. A correlation between the density-weighted critical sooting strain rate and $X_{F, 0}^{1 / 2} P$ was confirmed again (with the exception of the data point beyond $20 \mathrm{~atm}$ ), although a methodology different from previous studies was used to determine the critical strain rate $[85,573]$. 

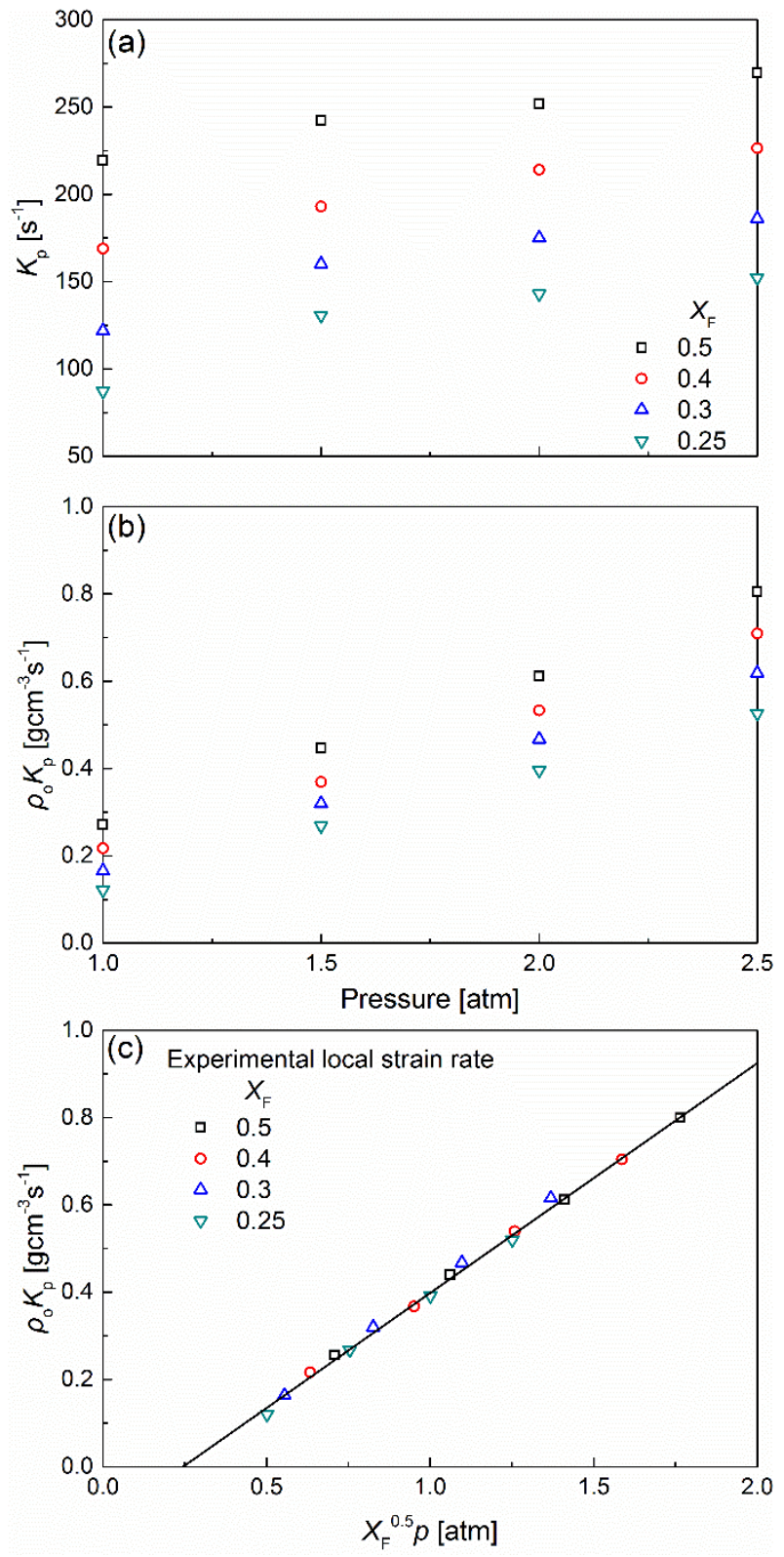

Figure 39. Effects of pressure on the critical strain rate (a) and density-weighted critical strain rate (b) for soot formation in counterflow diffusion flames. Also shown is the linear correlation between $\rho_{0} K_{c r}$ and $X_{F, 0}^{1 / 2} P(\mathrm{c})$. Reprinted from Du et al. [573] with permission of Elsevier. 

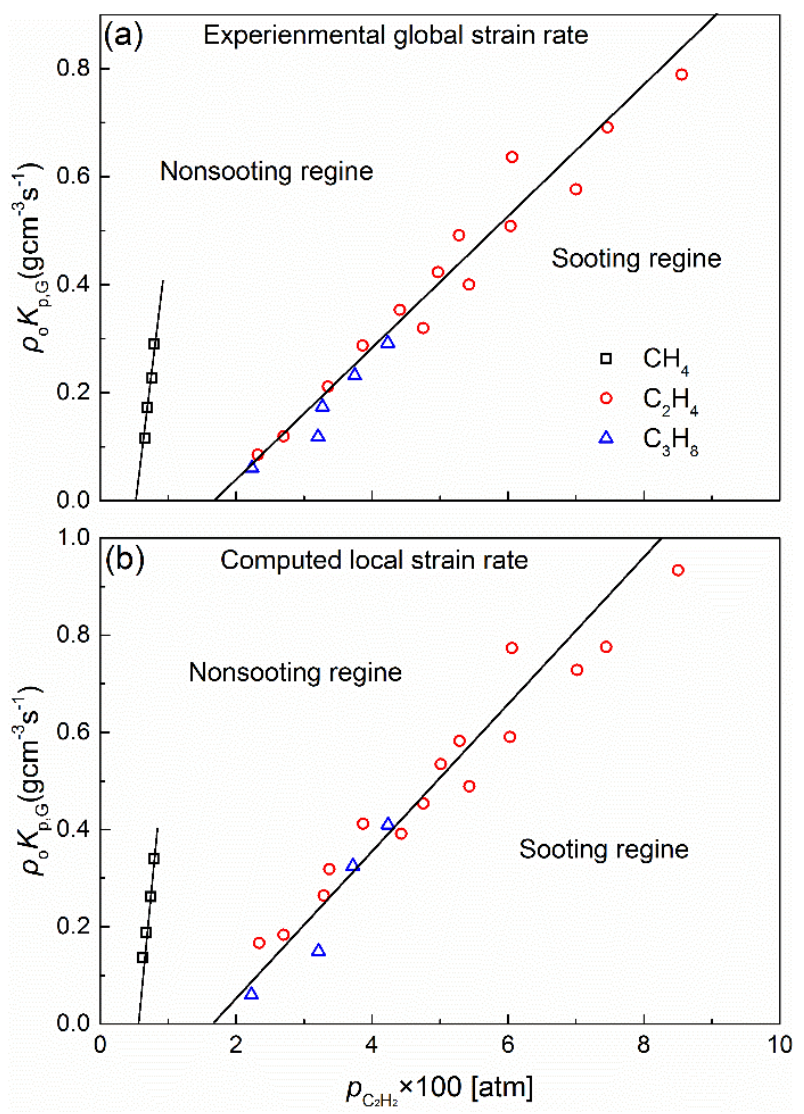

Figure 40. Correlations of density-weighted critical strain rate as a function of the peak $\mathrm{C}_{2} \mathrm{H}_{2}$ partial pressure. Reprinted from Sung et al. [85] with permission of Elsevier.

The effects of pressure on PAH formation were investigated by Böhm and Lacas [575] for methane-air CDFs from 1 to 6 bar with $X_{\mathrm{F}, 0}=1.0$ and with a global strain rate $K_{\mathrm{G}}=200 \mathrm{~s}^{-1}$. PAH concentrations were estimated experimentally by LIF signal intensities (excited at $283 \mathrm{~nm}$ and detected at around $315 \mathrm{~nm}$ ) and determined computationally by simulations with detailed chemistry. An approximate square dependence was observed for the peak PAH LIF signal with the increase of pressure (LIF intensity $\sim p^{2}$ ), consistent with the calculated maximum PAH concentration (summing up to pyrene) and previous experimental data obtained in premixed $\mathrm{C}_{2} \mathrm{H}_{4}$ flames. It should be pointed out that the flames studied by Böhm and Lacas [575] were close to the sooting limit but essentially non-sooting. Note that the increase in pressure also led to an increase in computed peak flame temperature $(2000 \mathrm{~K}$ at $1 \mathrm{bar}, 2100 \mathrm{~K}$ at $3 \mathrm{bar}$ and $2150 \mathrm{~K}$ at $6 \mathrm{bar})$. Thus, the authors commented that the higher PAH concentration at elevated pressures was not only an effect of pressure. It is 
relevant that the increase in peak temperature with pressure was also experimentally confirmed by Figura and Gomez [788] in methane CDFs using the technique of SiC thin filament pyrometry [790]; and it was shown that the increase can be more than the change of adiabatic flame temperature with pressure [636].

Comprehensive chemical speciation in high pressure CDFs was pioneered by Figura and Gomez [618]. In particular, with micro-probe sampling and subsequent GC-MS analysis the authors measured the axial profiles of major species, $\mathrm{C}_{2}-\mathrm{C}_{5}$ intermediate hydrocarbons as well as aromatic species, up to indene. The choice of flame conditions was based on the consideration of flame stability, adiabaticity, pressure tractability, and sooting level. Too much soot was undesirable as it would cause micro-probe clogging during the sampling process, while non-sooting flame would have rather low concentrations of PAHs, which was of special interest to the authors. Two series of flames at incipient sooting conditions were finally chosen for experiments, as well as numerical simulations using a detailed reaction mechanism accounting for PAH formation [791]. Flame series one featured a constant fuel mass fraction $\left(Y_{\mathrm{F}, 0}\right)$ of 0.122 , a stoichiometric mixture fraction $\left(Z_{\mathrm{st}}\right)$ of 0.408 and a global strain rate of $K_{\mathrm{G}}=57 \mathrm{~s}^{-1}$, while the pressure was varied from 1 to 8 bar. Flame series two covered only two flames at 8.55 and 25 bar, with $Y_{\mathrm{F}, 0}=0.0975, Z_{\mathrm{st}}=0.408$ and $K_{\mathrm{G}}=18.4 \mathrm{~s}^{-1}$. The reduction of $Y_{\mathrm{F}}$ in the second series was intended to avoid excessive soot loading at the high-pressure conditions (25 bar). A reduction of micro-probe outer diameter ( $\left.d_{\text {out }}\right)$ was shown to be necessary for probing CDF at increasingly higher pressures, if sufficient spatial resolution was to be achieved [623]. In fact, it was suggested that $\delta / d_{\text {out }}$ should be kept above 20 [623], where $\delta$ is the thickness of the mixing layer, which decreases as pressure increases. In flame series one, with increased pressure, the experimental peak mole fractions of $\mathrm{CH}_{4}, \mathrm{C}_{2} \mathrm{H}_{6}, \mathrm{C}_{3} \mathrm{H}_{8}, \mathrm{C}_{3} \mathrm{H}_{6}, \mathrm{n}-\mathrm{C}_{4} \mathrm{H}_{10}$ and 1- $\mathrm{C}_{4} \mathrm{H}_{8}$ decreased and those of 1,3- $\mathrm{C}_{4} \mathrm{H}_{6}, \mathrm{C}_{4} \mathrm{H}_{4}, \mathrm{C}_{4} \mathrm{H}_{2}, \mathrm{c}-\mathrm{C}_{5} \mathrm{H}_{6}$, methyl-cyclopentadiene, and all tested aromatic species, increased, while the $\mathrm{C}_{2} \mathrm{H}_{2}$ mole fraction remained comparable. The enhancements in sooting tendencies from increasing pressure in both flame series were evidenced by significant increases in indene mole 
fractions at the highest pressure conditions; this was attributed by the authors to the increases in reactant concentrations with pressure [618]. Besides providing quantified concentration data to assist kinetic model developments for high-pressure applications, an additional contribution of the work was an extensive discussion on scaling and the invariance of temperature-time history under different pressure conditions. The authors demonstrated that for a fixed strain rate, the mixing layer thickness $\delta$ of the CDF was proportional to $P^{-1 / 2}$. After normalizing the axial position and temperature by $\delta$ and peak temperature, respectively, the non-dimensionalized temperature profiles for flames at different pressures (but the same $Y_{\mathrm{F}, 0}, Y_{\mathrm{O}, 0}$ and $K_{\mathrm{G}}$ ) collapsed into a single line. It was further shown that the temperature-time history within the thermal layer would be invariant for flames at different pressures, provided that these flames have a constant strain rate. Such invariance provides tractability in comparing flames operated at different pressures, otherwise, variations in residence time would add to the pressure effects to an unknown extent $[618,623]$.

In a more recent study [636], the density-weighted global strain rates $\left(\rho K_{\mathrm{G}}\right)$, instead of strain rates $K_{\mathrm{G}}$, were kept constant for moderately sooting ethylene CDFs ranging 1-8 atm. SVF profiles were measured with LII excited at $532 \mathrm{~nm}$, and detected at around $450 \mathrm{~nm}$, calibrated against measurements with light extinction. Nozzle exit velocities (and thus $K_{\mathrm{G}}$ ) had to be progressively lower to counteract the pressure increase to maintain a fixed $\rho K_{\mathrm{G}}$. As expected, both peak local and integrated SVF increased with increased pressure. The pressure scaling factor $n$ was derived from the experimental data by plotting $\ln \left(f_{\mathrm{v}, \max } / f_{\mathrm{v}, \max , 0}\right)$ as a function of $\ln \left(P / P_{0}\right)$ and taking the slope, where the subscript 0 refers to a reference pressure state (e.g., at $1 \mathrm{~atm}$ ). It was found that the value of $n$ varied with $X_{\mathrm{F}, 0}$ and $X_{\mathrm{O}, 0}$, and for a fixed pair of $X_{\mathrm{F}, 0} / X_{\mathrm{O}, 0}, n$ was larger in cases with constant $\rho K_{\mathrm{G}}$ than in cases with constant $K_{\mathrm{G}}$. The authors also showed that the thickness of the soot zone and the thermal mixing layer became invariant with pressure, as long as $\rho K_{\mathrm{G}}$ was fixed, consistent with previous scaling arguments [618]. Based on this fact, the authors pointed out the importance of maintaining $\rho K_{\mathrm{G}}$-rather than $K_{\mathrm{G}}$ - at a constant value to study pressure effects. However, it may be worthwhile to point out that 
with $\rho K_{\mathrm{G}}$ fixed, the variation of flow residence time with pressure may complicate the isolation of pressure effects, as demonstrated by Figura and Gomez [618, 623].

Amin and Roberts [529] pioneered in-situ determination of the morphology of soot particles in CDFs at elevated pressures. Specifically, laser light extinction and two-angle scattering were used to measure SVF, primary particle size, and number density, along with aggregate size (average radius of gyration) and number density in ethylene CDF at 2-5 atm with $X_{\mathrm{F}, 0}=0.3$ and $X_{\mathrm{O}, 0}=0.21$. Note that $K_{\mathrm{G}}=30 \mathrm{~s}^{-1}$, rather than $\rho K_{\mathrm{G}}$ was kept constant. A special high-pressure vessel with four curved windows was built, providing $160^{\circ}$ of optical access to allow collection of scattering signals at various angles with respect to the incident beam. The results are summarized in Figure 41. Skewed profiles of SVF $f_{\mathrm{V}}$ (a) and scattering coefficient $K_{\mathrm{VV}}$ (b) can be seen, consistent with soot evolution in SF flames. With the increase in pressure, SVF (a), primary particle size $d_{\mathrm{p}}(\mathrm{c})$, and aggregate size $R_{\mathrm{g}}(\mathrm{d})$ were all increased, but the primary particle number density (c) decreased. The reduction of soot number density was explained by enhanced coagulation efficiencies, and it is noted that a pressure increase was reported to reduce coagulation efficiency in premixed flames [776]. Peak soot yield was found to be scaled with pressure raised to the power of 2.67 [529]. 

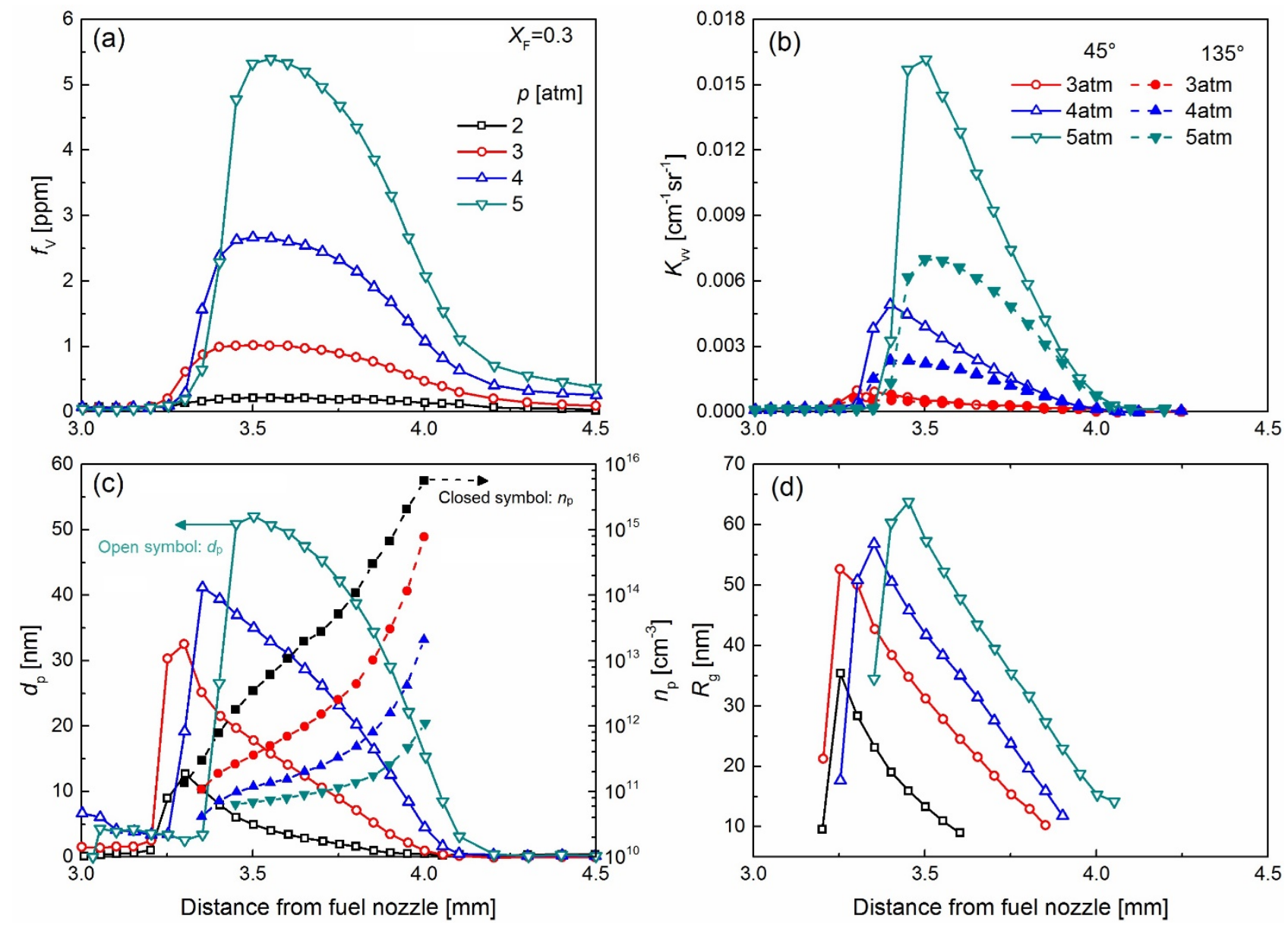

Figure 41. Sooting structures of ethylene counterflow diffusion flames at elevated pressures. Data taken from Amin et al. [529].

Carbone et al. [628] measured the mole fraction profiles of major and critical soot related intermediate species in $\mathrm{C}_{2} \mathrm{H}_{4}$ counterflow partially-premixed flames at 1, 4, and 8 atm. Three fuel side equivalence ratios $\left(\infty, 6.5\right.$ and 5.0) were tested at each pressure, while keeping $Z_{\mathrm{st}}$ and $K_{\mathrm{G}}$ constant for all the flames. For consistent temperature-time histories for the reactants (so that the effects of pressure could be better isolated), the peak flame temperatures at high-pressure flames were deliberately reduced by increasing dilution. Nevertheless, the benzene mole fraction was still seen to increase for partially-premixed flames with increasing pressure, despite the higher dilution level. Reduced back diffusion of $\mathrm{OH}$ radical was thought to be a contributor to the enhanced soot precursor formation, aside from the pressure effects in increasing reactant concentrations [618]. Through kinetic 
simulations, it was observed that with increasing pressure, the $\mathrm{C}_{2}+\mathrm{C}_{4}$ pathways for benzene formation became increasingly more important than the $C_{3}+C_{3}$ pathways.

More recently, Gleason et al. [490] complemented the experimental database on chemical structures of high-pressure counterflow flames with soot volume fraction measurements. Flame conditions were carefully selected to make sure that the normalized temperature-time history was identical, regardless of pressure. The measurement result showed that soot volume fraction increased by two orders of magnitude as pressure increased from 1 to $4 \mathrm{~atm}$ while peak flame temperature was kept unchanged. Further analysis on soot growth rate also demonstrated that the activation energies for high-temperature soot growth are the same at different pressures.

This discussion on pressure effects concludes by affirming the great necessity for more quantified $\mathrm{PAH}$ and soot characterization in high-pressure CDFs, with well-controlled conditions, especially for practical liquid fuels and their surrogates. Both challenges and opportunities exist for investigations on how, and to what extent pressure-dependent reactions affect soot formation, a topic that has been largely unexplored by existing studies.

\subsection{Effects of temperature on soot formation}

The qualitative and quantitative dependence of soot formation on temperature has received extensive research attention, justifiable by the fact that soot formation is a highly temperaturesensitive process. Temperature is a major influence not only on the formation of soot particles, but also on their oxidation. Early relevant investigations generally focused on the effects of temperature on critical $\mathrm{C} / \mathrm{O}$ ratios $\left((\mathrm{C} / \mathrm{O})_{\mathrm{cr}}\right)$, or critical equivalence ratios $\left(\phi_{\mathrm{cr}}\right)$ for soot appearance in premixed flames [98, 685, 772, 778, 792-795], and sooting height, or SVF profiles, in jet diffusion flames [96, $728,796,797]$.

For burner-stabilized premixed flames, it was generally observed that $\phi_{\text {cr }}$ first decreases (i.e., increase of sooting tendency) and then increases with the progressive increase in temperature, 
exhibiting an interesting non-monotonic variation [772]. At a constant $\mathrm{C} / \mathrm{O}$ ratio, $\mathrm{SVF}$ was also seen to peak at a certain temperature, and either an increase or a decrease from this temperature would decrease soot formation $[97,772,794]$. These findings can be partially understood by the competing effects of temperature on soot precursor formation and on oxidative destruction. In the low end of the temperature range, an increase in temperature would result in faster fuel decomposition to soot precursors and an enhancement of the molecular growth processes towards PAHs and soot particles. However, further increase in temperature would result in a faster increase in the oxidation rates of soot precursors/particles than in the rates for their formation [796]. Also, reduced surface reactivity [597] and decreased thermodynamic stability of soot precursors [141] can play important roles in reducing soot formation at high temperatures.

For jet diffusion flames, Glassman and coworkers $[96,796]$ showed that sooting height decreases with the increase in adiabatic flame temperature, demonstrating a qualitative trend in these flames that increases in temperature lead to an enhancement of the sooting tendency. Note that the adjustment of flame temperature was achieved by the authors through inert dilution $\left(\mathrm{N}_{2}\right.$ or $\left.\mathrm{Ar}\right)$ in the fuel stream, which may affect soot formation through both flame temperature and concentration modifications. Nevertheless, the authors proved that the effect of temperature is dominant and fuel dilution plays only a secondary role. Although this conclusion seems to be consistent with the sooting limit curve obtained for SF CDFs (e.g., Fig. 26, with relatively large $X_{\mathrm{F}}$ ) where temperature dominates the critical condition of soot formation, in other cases it was reported that dilution can have important and even greater effect than temperature $[95,700]$.

In diffusion flames, soot inception and growth occur on the fuel side of the flame, where the concentration of oxidizing species is rather low. Without the competing effect of oxidation, an increase in flame temperature is expected to enhance the rates of soot precursor formation, as well as those of particle inception and surface growth, leading to a monotonic increase of sooting tendency. Note that for over-ventilated coflow diffusion flames, oxidation occurs later in the post-flame zone 
as heterogenous reactions, which will notably affect ultimate soot emission, but not the critical sooting condition [796]. Also note that fuel pyrolysis in diffusion flames is not the same as in a shock tube, where it was shown that soot yields have non-monotonic variation with temperature [798]. In a shock tube, pyrolysis occurs at uniform temperature, and if this temperature is too high, the thermodynamic stability of soot precursors decreases, and soot production is reduced $[141,798]$. On the other hand, there is always a spatial profile of temperature in diffusion flames, extending from boundary temperatures in the fuel/oxidizer inlets all the way to peak flame temperature. Fuel pyrolysis and soot inception can always be efficient locally at a location with intermediate temperatures. As a result, the sooting tendency in diffusion flames generally increases monotonically with temperature. Beltrame et al. [576] showed that in a soot formation CDF with undiluted methane as the fuel stream, peak SVF increased monotonically as $X_{\mathrm{O}, 0}$ increased from $21 \%$ all the way to $100 \%$ (although it should be noted that increases of $X_{\mathrm{O}, 0}$ reduce dilution as well as increasing temperature).

In fact, the variation of temperature of diffusion flames was frequently achieved by adjusting the amount and type of diluents in both fuel and oxidizer streams, so that the effects of dilution were likely to interfere with the effects of temperature. Therefore, dilution effects must be isolated if a quantitative correlation between soot formation and temperature is to be obtained. In this regard, Axelbaum et al. [74] adjusted the flame temperature of CDFs by the substitution of volume flow rate of $\mathrm{N}_{2}$ in the oxidizer stream and an equal volume flow rate of Ar. Since Ar has a lower heat capacity than $\mathrm{N}_{2}$, its substitution resulted in an increase in peak flame temperature. Since the mole fraction of the inert diluent was not affected, temperature could be varied without affecting dilution level. Using such an approach, the effects of dilution and temperature can be separated. In practice, flames with different levels of fuel stream dilution can be compared at a fixed peak temperature; and flames with different peak temperatures can also be compared at constant $X_{\mathrm{O}, 0}$ and $X_{\mathrm{F}, 0}$. Results from these types of studies were discussed in a previous section on inert dilution and will not be repeated here. 
A well-controlled peak temperature does not necessarily mean a controlled spatial temperature profile [74], and the latter may be of importance for the sooting process in SF CDFs, where the particle growth region is spatially separated from the peak flame temperature region. Ideally, the effects of temperature on soot formation should be studied with a well-controlled, well-characterized temperature-time history (i.e., not only the peak temperature) which the soot particles may experience. Towards this goal, Carbone et al. [628] demonstrated the importance of keeping the stoichiometric mixture fraction $\left(Z_{\mathrm{st}}\right)$ and the global strain rate $\left(K_{\mathrm{G}}\right)$ constant when perturbing peak flame temperatures. By doing this, the position of the flame front relative to the gas stagnation plane-as well as the flame thickness-can be kept constant so that after normalizing the temperature with respect to its peak value, the normalized temperature-convective time history remains similar among flames with different peak temperatures. In other words, the temperature-time profiles become selfsimilar, so the effects of peak temperature could be effectively isolated $[489,490]$. Note, in order to achieve peak temperature variation while keeping $Z_{\text {st }}$ constant, the dilution levels in both the fuel and oxidizer streams must be altered, although this additional complexity caused by the variations of dilution level may be insignificant if the required temperature range is moderate. In particular, Gomez and coworkers [489] showed that in order for the peak temperature of ethylene CDF to vary from 1890 to $2186 \mathrm{~K}$ (variation of around $300 \mathrm{~K}$ ), the fuel mass fraction must increase from 0.30 to 0.39 , while the oxygen mass fraction in the oxidizer stream must increase from 0.23 to 0.30 . The measured axial profiles of temperature, SVF and soot production rate for this series of ethylene CDFs are shown in Figure 42. 

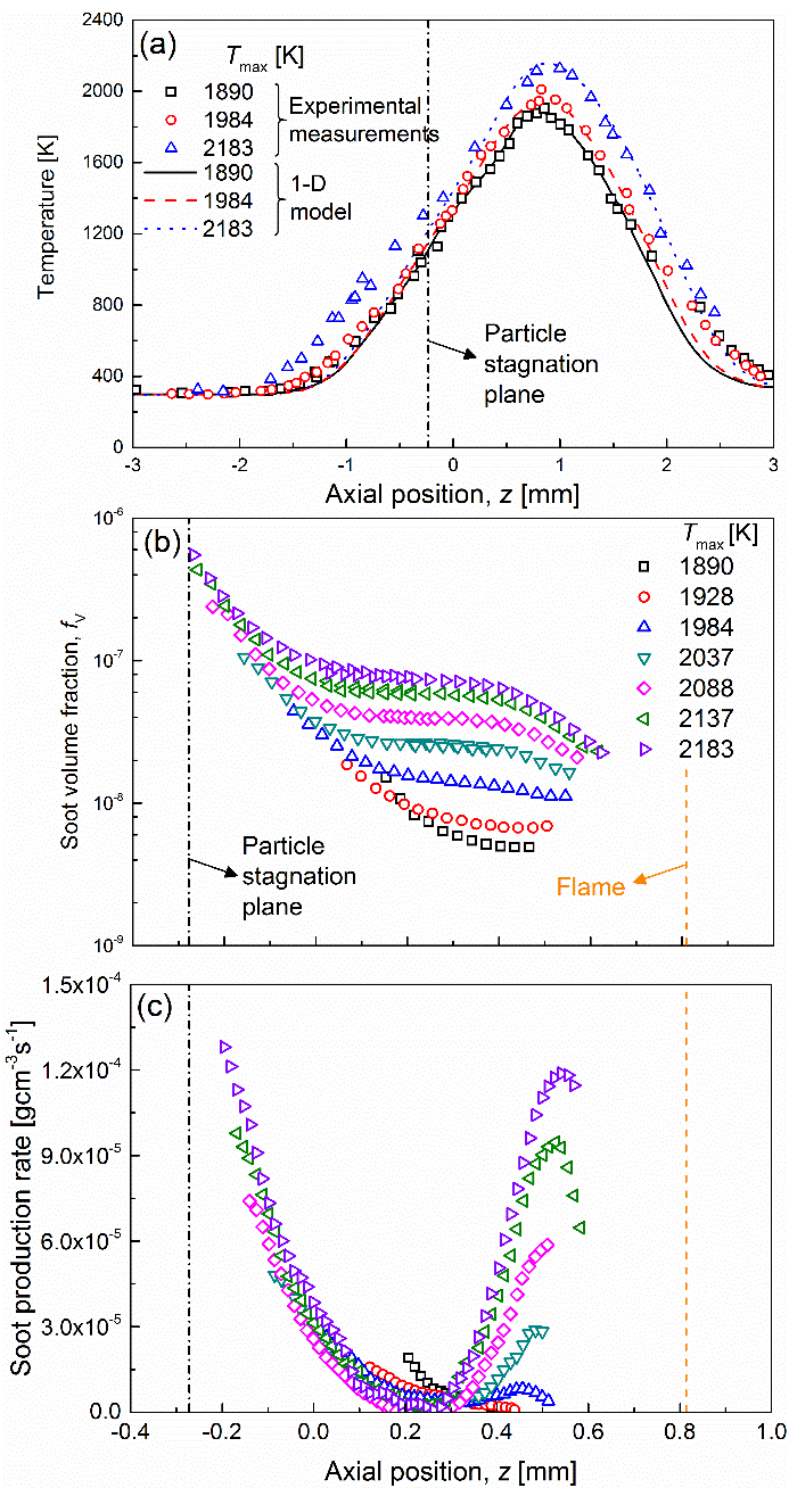

Figure 42. Axial profiles of temperature (a), SVF(b), and soot production rates (c) for ethylene CDFs with well-controlled convective time-temperature history. Adapted from Carbone et al. [489] with permission of Elsevier.

As can be seen in Figure 42 (a) and (b), although the temperature profiles are self-similar, the shape of the SVF profiles vary among the different peak temperature cases. In these flames, the SVF begins to increase in a region close to the flame front and then continues to grow as they are convected towards the particle stagnation plane, exhibiting common features for SF flames. However, the rates of soot growth vary notably among the flames. As shown clearly in Figure 42 (c), for cases with lower peak temperature, the initial growth rates are also lower. Since this initial growth occurs in a high 
temperature region $(Z \sim 0.5 \mathrm{~mm})$, where HACA surface reactions are expected to be efficient, their decreasing rates, with decreasing peak temperatures, are consistent with the high activation energy characteristics of the HACA reaction scheme. As particles are transported towards the stagnation plane, excessive temperature reduction causes the soot growth rates to fall quickly to nearly vanishing values. A somewhat unexpected observation was a sharp increase in soot growth rates in regions very close to the stagnation plane, where the temperature was below $1600 \mathrm{~K}$. The authors suggested a possible ion-induced nucleation mechanism to explain this. Although this seems to be qualitatively consistent with the modelling results of Wang et al. [140], where nucleation reaction and physical addition of PAH were seen to contribute to soot growth in the proximity of the particle stagnation plane, the comparable peak level of this low-temperature growth rate with that of the hightemperature HACA rate was not predicted by Wang et al. More studies, involving both numerical and experimental efforts, are needed to shed light on the mechanisms of this interesting lowtemperature soot growth. It is also important to point out that Sirignano et al. [89] also found evidence of low temperature soot growth and demonstrated that soot particles can also exist in the fuel side of the particle stagnation plane.

\subsection{Effects of strain rate on soot formation}

In Section 4.3, we discussed how strain rate may influence the overall sooting structure of CDFs. Here we intend to complement that discussion with a more comprehensive review on strain rate effects on $\mathrm{PAH}$ and soot formation. Because $\mathrm{PAH} /$ soot formation is a kinetically-driven rate-limiting process, quantitative investigations on the correlation between sooting characteristics and strain rate in CDFs can help explain the kinetics of soot formation.

A second motivation for studying strain rate effects is the link between diffusion flamelet and turbulent flame, the latter is somewhat difficult to model due to the large variations of temporal and spatial scales involved. The task becomes even more challenging when soot formation chemistry is 
considered, which by itself includes a large number of reactions, spanning a wide range of reaction time scales. The flamelet model, as pioneered by Peters [106], provides a tractable approach for simulating turbulent diffusion flames with complex chemistry. In such a model, local structures of the turbulent reaction zones are approximated by strained flamelets, which are to be convected and stretched by the turbulent flow fields. A key parameter characterizing diffusion flamelets is scalar dissipation rate $(\chi)$, closely related to, and with the same unit (reciprocal of time) of strain rate. In fact, the structures of steady flamelets can be solved entirely in the mixture fraction space once $\chi$ is specified [799]. In this context, a CDF is an appropriate model because of its relevance to many physics postulated for diffusion flamelets. The strain rate of CDF can be linked to $\chi$ of diffusion flamelet in a straightforward way. Besides, efforts needed to systematically vary the strain rate of $\mathrm{CDF}$ are minimal as it can be achieved by simply adjusting the nozzle exit velocities. This is in contrast to coflow flames, in which the strain rate is difficult to control and usually too small to be a representative of turbulent flames [114].

It is important to highlight here that due to the slow kinetics involved in PAH/soot formation, sooting process in highly transient flames can have significant unsteady effects. The above-mentioned (steady) flamelet approximation is not able to predict the formation of soot, although it may be used with success in predicting the overall local structure of turbulent flames (i.e., major flame products). As a result, it is of practical interests to investigate soot formation under unsteady strain rate conditions. Section 5.5.2 is dedicated to this matter.

\subsubsection{Strain rate effects on sooting behaviors in steady CDFs}

A number of studies have investigated how strain rate affects PAH formation, both experimentally $[91,114,585]$ and computationally $[91,92,114,800]$. The experimental techniques that were used to evaluate PAH concentrations were either gas chromatography or LIF; while chromatography can provide quantified mole fractions of individual PAH species, LIF can only provide fluorescence 
intensities that qualitatively represent a class of PAHs in a certain size range. These studies generally demonstrated that as the strain rate increases, peak flame temperature, $\mathrm{C}_{2} \mathrm{H}_{2}$ and $\mathrm{C}_{3} \mathrm{H}_{3}$ decrease moderately, and the mole fractions of aromatic species decrease more significantly. In addition, $\mathrm{PAH}$ species with larger molecular sizes are more sensitive to strain rate. An example is shown in Figure 43 for $\mathrm{C}_{2} \mathrm{H}_{4} \mathrm{CDF}$ with $X_{\mathrm{F}, 0}=1.0$ and $X_{\mathrm{O}, 0}=0.25$ [91], where both numerical and experimental data are provided. A similar trend was also observed in a study of $\mathrm{CH}_{4}$-air $\mathrm{CDF}$, where the investigated strain rate spanned a much larger range (i.e., $K_{\mathrm{G}}$ from $100 \mathrm{~s}^{-1}$ all the way to $600 \mathrm{~s}^{-1}$, close to extinction) [114]. More quantitative $\mathrm{GC}$ speciation analysis [590] of a $\mathrm{C}_{2} \mathrm{H}_{2} \mathrm{CDF}$ with $X_{\mathrm{F}, 0}=0.375$ and $X_{\mathrm{O}, 0}=$ 0.220 (balanced by Ar) showed that, as the effective strain rate $K_{\mathrm{E}}$ increased from 38 to $50 \mathrm{~s}^{-1}$, the peak mole fraction of benzene (A1) decreased from $2 \times 10^{-4}$ to $8 \times 10^{-5}$, by approximately $40 \%$. This is to be compared against naphthalene $\left(\mathrm{A} 2,4 \times 10^{-5}\right.$ to $\left.5 \times 10^{-6}\right)$ and pyrene $\left(\mathrm{A} 4,3 \times 10^{-5}\right.$ to $\left.4 \times 10^{-7}\right)$, the mole fractions of which exhibited a decrease of one and two orders of magnitude, respectively.

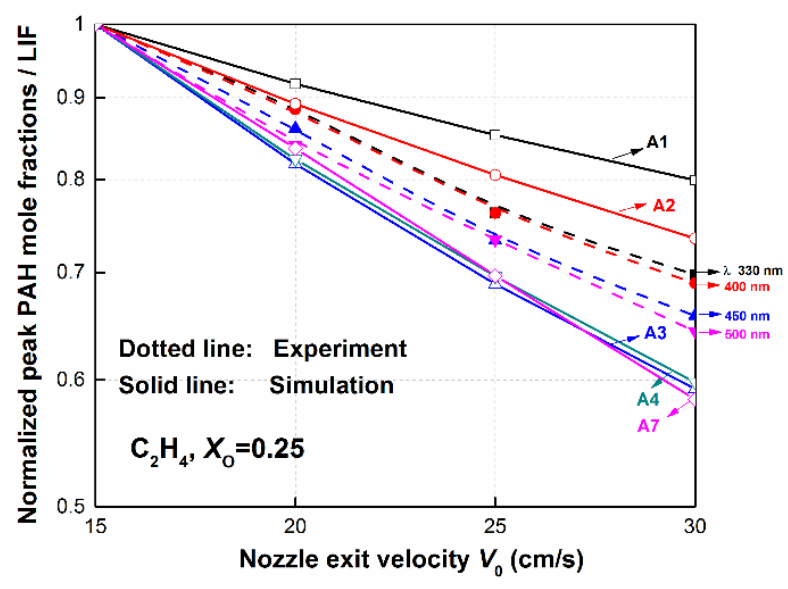

Figure 43. Effects of strain rate on PAH formation in ethylene counterflow diffusion flames. Both computed peak PAH mole fractions and experimental peak PAH LIF intensities are shown. Note PAH LIF signals detected at larger wavelengths are expected to represent PAHs with larger molecular sizes. Reprinted from Wang and Chung [91] with permission of Elsevier.

The effects of strain rate on flame and PAH chemistries result from several mechanisms. First, the flame intensity (and thus peak flame temperature) are expected to decrease with the increased strain rate, primarily because of the reduced Damköhler number. Since the temperature-dependent fuel 
pyrolysis process is essential in diffusion flames to produce small molecular precursors (i.e., $\mathrm{C}_{2} \mathrm{H}_{2}$, $\mathrm{C}_{3} \mathrm{H}_{3}, \mathrm{C}_{4} \mathrm{H}_{3}$, etc.) for PAH growth, decreased temperature would lead to lower $\mathrm{PAH}$ formation rate; Second, even with abundant small molecular precursors, the growth of PAH is a slow rate-limiting process and would be significantly inhibited by decreased residence time. Since larger PAHs grow sequentially from smaller ones, this also explains why larger PAHs are progressively more sensitive to strain rate; Third, the general scaling argument [81] shows that the increase in strain rate would reduce the characteristic thickness of the flame, resulting in the originally-separated pyrolysis zone and high temperature $\mathrm{OH}$-oxidizing zone being pushed closer together. As a result, concentrations of oxidizing species in the fuel pyrolysis zone tend to increase, causing increased fuel oxidation to form sequentially $\mathrm{CH}_{2} \mathrm{O}, \mathrm{CHO}$ and $\mathrm{CO}$. Note, however, this last mechanism is expected to be important only at very high strain rates (i.e., close to extinction) [114].

The first quantitative measurement of the variation of SVF with strain rate was conducted in CDFs stabilized around a cylindrical porous burner using laser light extinction and scattering [554]. Similar experimental studies were later performed in counterflow burners with opposing screened, or contoured, nozzles $[78,91,576]$. Much as expected, these studies generally showed that the peak SVF exhibited a monotonic decrease as the strain rate increased, as has already been discussed in

\section{Section 4.3.}

It is logical to expect that fuels with distinct molecular structures will have different sooting rates when burned under similar conditions. For instance, it is widely accepted that benzene formation is a critical step, for being rate-limiting, toward the formation of soot in flames of small aliphatic fuels. On the other hand, the situation is obviously different for fuels that already contain aromatic rings (benzene formation can be bypassed). Similar arguments can be applied even to different fuels belonging to the same class, considering that different fuel molecules lead to pyrolysis/oxidative intermediate species that contribute to soot precursor formation through different channels and at different rates. Variations in the sooting rate of different flames are expected to lead to variations in 
the sensitivity of the final soot concentrations to strain rate. Decroix and Roberts [801] found the rates of decrease in peak SVF to be higher for $\mathrm{C}_{3} \mathrm{H}_{8}$ than in $\mathrm{C}_{2} \mathrm{H}_{4}$ CDF. Similar experimental results were obtained by Wang and Chung [91], with additional measurements for $\mathrm{C}_{3} \mathrm{H}_{6}$ and $\mathrm{C}_{2} \mathrm{H}_{6}$ flame. Their results showed that the peak $\mathrm{SVF}$ in $\mathrm{C}_{2} \mathrm{H}_{6}$ flame had a higher sensitivity than $\mathrm{C}_{3} \mathrm{H}_{8}$ flame to strain, and $\mathrm{C}_{3} \mathrm{H}_{6}$ flame had the lowest sensitivity. In addition, numerical simulations [92] showed that the dependence of peak SVF to strain rate can be fitted into a power law relation: $f_{\mathrm{v}, \max }\left(K_{\mathrm{G}}\right) / f_{\mathrm{v}, \max }\left(K_{\mathrm{G} 0}\right)=$ $\left(K_{\mathrm{G} 0} / K_{\mathrm{G}}\right)^{a}$ where $K_{\mathrm{G} 0}$ is a reference global strain rate and the subscript $a$ is the exponent, with higher values indicating higher sensitivity of SVF to strain rate. The value of $a$ was found to be fueldependent, and it was 0.88 for benzene flame, much smaller than the value of 1.2 and 2.9 for ethylene and methane flames, respectively [92].

A more interesting observation [91] is that, although the sensitivity of soot loadings to strain rate depend on fuel type at a fixed flame condition $\left(X_{\mathrm{F}, 0}, X_{\mathrm{O}, 0}\right)$, once the peak SVF is adjusted (for instance, by varying $X_{\mathrm{O}, 0}$ ) to be similar among these different fuels at a given reference strain rate, the sensitivity of peak SVF to strain rate becomes reasonably independent of fuel type. This point is clarified in Figure 44, where the correlation between peak SVFs and their slope of decrease with strain rate (represented by nozzle exit velocity $V_{0}$ in the figure) are shown. Although the data were collected for various fuels, they fall on the same line, demonstrating that the peak SVF at a reference strain rate (not fuel type) dominates the strain rate sensitivity. This interesting result seems to indicate an inherent similarity in the soot formation processes among different fuels. It should be remembered, however, that this conclusion is only drawn from the four aliphatic fuels tested in Figure 44, and further investigations are necessary to test whether it can be generalized to other fuels. It is also noted that this result is consistent with the sooting sensitivity index (SSI) data [620], developed to quantify the dependence of sooting tendency of a particular fuel to strain rate variations. 


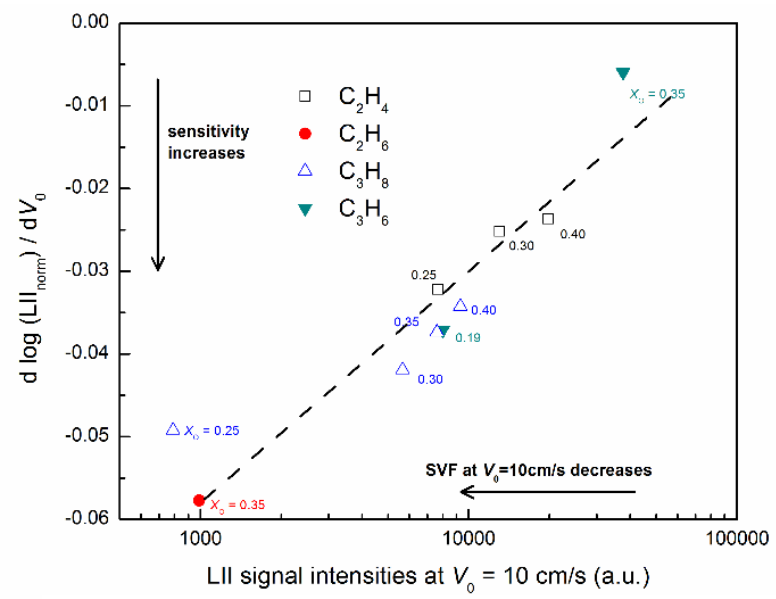

Figure 44. Correlation between soot loading and its sensitivity to strain rate. Reprinted from Wang and Chung [91] with permission of Elsevier.

In addition to global soot loading, as characterized by SVF, the effects of strain rate on the details of particle size distribution were also investigated. Numerical simulation with a sectional method [92] showed that in both benzene and ethylene SF CDFs, the percentage contribution of the number of small particles $(\sim 1.7 \mathrm{~nm})$ to the total soot number density was only marginally affected, but those of larger particles decreased notably with the increase in strain rates.

\subsubsection{Soot formation in CDFs subject to unsteady strain rates}

The preceding discussions focused on sooting behaviors at steady conditions. For application in turbulent combustion modelling through the flamelet approach, unsteady effects are also important [802]. In a turbulent environment, the mean strain rate of flamelets is established from the largerscale eddies, while fluctuations around the mean values and associated unsteady effects can be caused by smaller eddies, with turnover time comparable to the diffusion time scale of the flamelets [803805]. CDF is an ideal flame configuration to study unsteady strain rate effects because the unsteadiness can be established experimentally by imposing oscillation on the nozzle exit velocity. Numerically, the quasi-one dimensionality makes time-resolved simulation with detailed chemistry much more efficient. 
Before addressing soot formation in unsteady CDFs, a general understanding of global flame responses to oscillating strain rates is helpful. Relevant studies on the dynamics of unsteady CDFs [804, 806-812] showed that for small oscillation frequencies of nozzle exit velocity (i.e., strain rate), the flame responds to the oscillations in a quasi-steady manner (characterized by heat release rates, temperature, or $\mathrm{OH}$ concentration profiles). As the oscillation frequency increases, some transient behavior (e.g., phase lag, reduced response) begin to develop. With further increase in oscillation frequency, the flame may fail to respond altogether. The cutoff frequency that differentiates between quasi-steady and transient responses was also observed to increase in cases with stronger burning [808]. An example of the variation of maximum flame temperature with oscillation frequency can be seen in Figure 45. Through a time-resolved simulation with detailed chemistry, Egolfopoulos and Campbell [805] provided a physical interpretation of the above observations: On either side of the reaction zone in a $\mathrm{CDF}$, there is a hydrodynamic zone, followed by a diffusion zone in which diffusion is the dominant mechanism for transporting fuel/oxidizer toward-and heat away from-the reaction zone. Although the effects of oscillation in the nozzle exit velocity can be propagated nearly unaltered through the hydrodynamic zone, the oscillation can only be sensed indirectly by the flame through the effects that far-field disturbances induce on the diffusion processes. It was further shown that the exit velocity oscillation in CDF creates an oscillation of mass fraction of the reactants. In this regard, mass diffusion of the reactants towards the reaction zone is the dominant mechanism controlling the flame response to oscillating boundary conditions. The authors proposed an analogy between the unsteady CDF and the Stokes second problem, in which the fluctuating velocities induced by an oscillating plate in an initially stagnant flow (through momentum diffusion) become attenuated with the increase in the distance away from the plate $(z)$ and the oscillation frequency $(\omega)$. The amplitude of velocity fluctuation in Stokes second problem is controlled by the non-dimensional Stokes parameter $\eta_{\mathrm{z}}=z(\omega / 2 v)$, where $v$ is the fluid kinematic viscosity. Similarly, it was shown that the amplitude of peak temperature fluctuation in unsteady CDFs is controlled by $\eta_{\mathrm{K}}=\delta(\omega / 2 D)^{1 / 2} \approx$ 
$\left(\omega / 2 K_{\mathrm{G}}\right)^{1 / 2}$, where the latter approximation is supported by the dependence of the diffusion zone thickness $\delta$ on the diffusivity $D$, and the global strain rate as $\delta \approx\left(D / K_{\mathrm{G}}\right)^{1 / 2}$. The flame response would decrease as $\eta_{\mathrm{K}}$ increased, either by increasing frequency or reduced strain, as shown in Figure 46, where the flame response of many different flames collapsed into a single line with $\eta_{\mathrm{K}}$. Physically, a decrease in the global strain rate can lead to increases in diffusion thickness so that the attenuation in flame responses are expected to be stronger. The increase in frequency would be accompanied by disturbances with shorter wavelength, causing larger gradients and diffusive fluxes-again dampening flame responses.

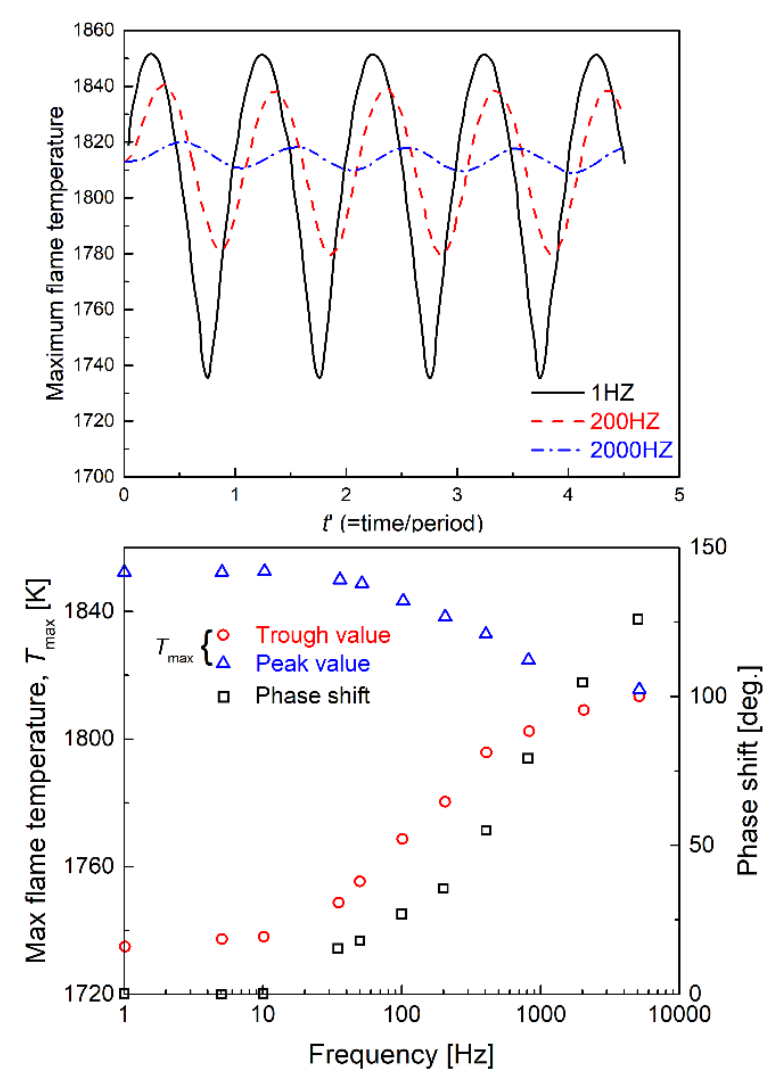

Figure 45. Oscillations in peak flame temperature of CDFs subject to oscillating strain rates at various frequencies (a) and frequency response of the amplitudes of maximum flame temperature and phase shift (b). Data taken from Egolfopoulos and Campbell [805]. 


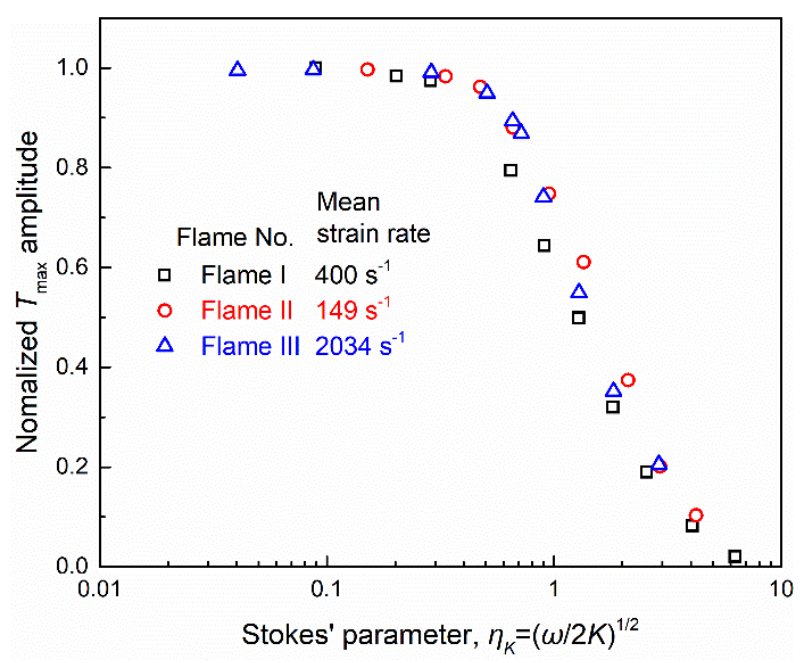

Figure 46. Correlations between the amplitude of peak temperature fluctuation (normalized its corresponding quasi-steady values) with relevant Stokes parameter. Data taken from Egolfopoulos and Campbell [805].

With overall flame behaviors under unsteady conditions clearly explained by Egolfopoulos and Campbell [805], more recent studies focused on how PAH and soot formation are affected by unsteadiness $[395,577,585,593,598,630,801]$. The response of $\mathrm{PAH} /$ soot formation to strain rate oscillations does not necessarily follow that of the peak flame temperatures or heat release since: 1) PAHs/soot are typically formed in the fuel rich pyrolysis zone that lie inside the diffusion zone of CFDs, compared to the thin reaction zone where most heat release occurs; 2) The chemical time scale for PAH/soot formation is notably larger than the main heat release reactions; 3 ) large PAHs and soot particles have very small diffusivity compared to gas-phase species. Time-resolved in-situ SVF measurements in unsteady CDFs were first performed by Decroix and Roberts [801] using LII. The oscillation in strain rate was experimentally achieved by driving two loudspeaker diaphragms, installed respectively at the end-faces of two plenums; through each plenum, the fuel and oxidizer were introduced to the nozzle outlets. Sine-wave driving voltages were used so that the global strain rate $\left(K_{\mathrm{G}}\right)$ varied in a sinusoidal manner around a prescribed mean value. Experiments were done at four initial $K_{\mathrm{G}}$ ranging from 15 to $90 \mathrm{~s}^{-1}$, with unsteady forcing at frequencies from 25 to $200 \mathrm{~Hz}$, and at both low and high amplitudes. Results showed that maximum soot SVF (global maximum in terms 
of both temporal and spatial variation) for the unsteady flames was generally higher than their steady counterparts, this enhancement was seen to be most prominent in cases of low forcing frequency and high amplitude. The SVF responses were much attenuated with high-frequency oscillations. For example, an increase by a factor of 3.4 in peak SVF was observed for propane CDF oscillated at 25 $\mathrm{Hz}$ around a mean $K_{\mathrm{G}}$ of $60 \mathrm{~s}^{-1}$ while soot production became nearly insensitive for oscillation frequencies larger than $100 \mathrm{~Hz}$. Furthermore, significant phase lag between the peak SVF peaks and strain rate troughs were noticed.

Because PAHs are the precursors of soot, their behavior in unsteady flames is particularly important for a better understanding of soot formation. For this reason, planar LIF was used to detect spatially- and temporally-resolved PAH concentrations for unsteady CDFs [585]. The LIF signals were detected at different wavelengths to represent PAHs of different size classes, with lower wavelengths corresponding to smaller PAHs. As expected, the results showed that PAH concentrations only responded to low frequency oscillation, becoming insensitive to oscillations beyond a critical value, which depends on PAH sizes and several other flame conditions like fuel type and mean strain rate. For larger PAHs, this critical frequency was lower, which was reasonable since the formation of larger PAHs requires the presence of smaller ones. However, an unexpected finding was that the critical frequency was smaller for flames with a higher mean strain rate. For example, with an initial strain rate $\left(K_{\mathrm{L}}\right)$ increasing from 23 to 44 , and then to $74 \mathrm{~s}^{-1}$, the critical frequency for large PAH responses decreased from 200 to 50 , and then to $30 \mathrm{~Hz}$. This is in sharp contrast to the scaling laws proposed by Egolfopoulos and Campbell [805] in which a normalized parameter $\eta_{\mathrm{K}}=$ $\left(\omega / 2 K_{\mathrm{G}}\right)^{1 / 2}$ controlled the amplitudes of the flame responses (i.e., flame temperature) to the oscillating strain rate. According to the definition of $\eta_{\mathrm{K}}$, the critical frequency would be larger for flames with higher $K_{\mathrm{G}}$. A physical interpretation (mentioned above) is that the thickness of the diffusion zone would be reduced so that free-stream oscillation could propagate into the reaction zone with less damping in flames with a higher strain rate. The notable deviation between the response of the PAH 
concentrations and this scaling law for overall flame properties (i.e., peak temperature) clearly demonstrates that diffusion-limited responses alone are not sufficient to explain the PAH behavior observed under unsteady conditions. The relatively larger time scale for $\mathrm{PAH}$ formation compared to that of the main heat release reaction is likely to play a role. It is noteworthy that although the authors in the original publication [585] attempted to correlate $\mathrm{PAH}$ with $\eta_{\mathrm{K}}$, the scatter of the data was fairly clear.

Besides the critical frequency, the amplitudes, symmetries and phase lags of the variations in $\mathrm{PAH} /$ soot concentrations subject to unsteady strain rates are also important. Figure 47 shows the peak, trough and mean values, as well as phase lags for the PAH mole fractions as a function of oscillating frequencies [593]. Clearly, the phase lags are always close to zero at low frequencies, indicating quasi-steady responses. However, the peak and trough values for PAH/soot concentrations are not symmetric around the mean values. The asymmetry becomes more significant for larger PAHs-and most notably for soot. More interestingly, the mean PAH/soot concentrations (averaged over an entire oscillating period) are higher than the steady state value, consistent with the experimental data of Decroix and Roberts [585]. The asymmetry and the resulting increase in the average $\mathrm{PAH} /$ soot concentrations in the unsteady flames may be attributed to the fact that $\mathrm{PAH} / \mathrm{soot}$ formations are more sensitive to the variations in strain rate (and thus residence time) at lower strain rate instants. This is more or less consistent with the findings in steady flames. For example, Beltrame et al. [576] showed that the peak SVF of a $\mathrm{CH}_{4}-\mathrm{O}_{2} \mathrm{CDF}$ decreased by around $50 \%$ as the strain rate increased from 20 to $40 \mathrm{~s}^{-1}$, while a further increase of strain rate by $20 \mathrm{~s}^{-1}$ only resulted in a decrease of peak SVF by $28 \%$. 

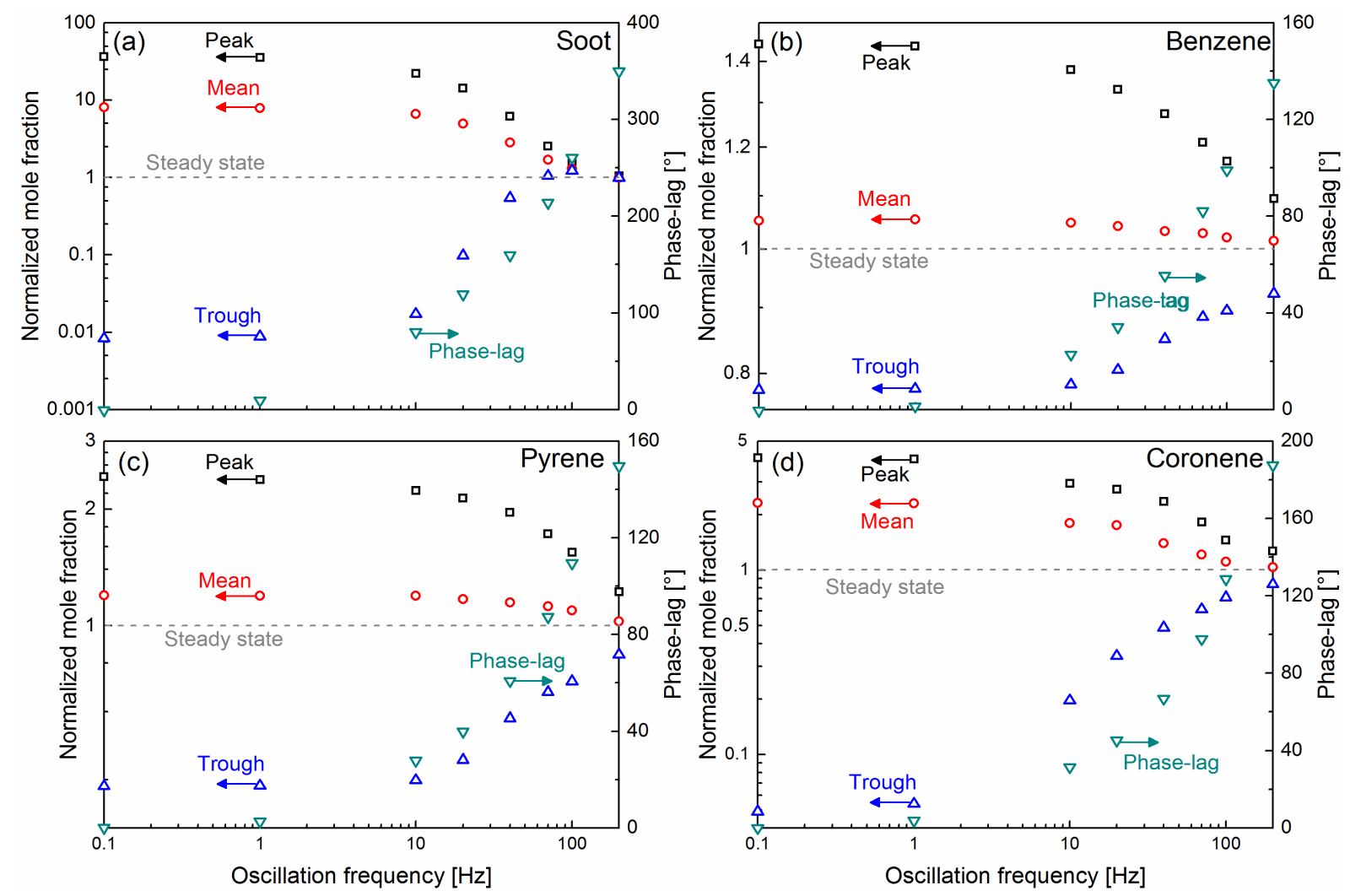

Figure 47. Detailed frequency responses of induced oscillation amplitude and phase-lag of soot and its precursors (benzene, pyrene and coronene). Reprinted from Cuoci et al.[395] with permission of Elsevier.

As oscillating frequency continues to increase, notable phase lag develops between PAH/soot concentrations and strain rates. The phase lag was also seen to be larger for species with a larger characteristic reaction time scale [395, 630]. Cuoci et al. [395] explained this by referring to the diffusion zone through which free-stream oscillations must pass to affect the reaction zone, and the species-dependent responses were attributed to the different diffusion time of each species. Following analysis by Egolfopoulos and Campbell [805], the authors also tried to correlate the predicted oscillation amplitudes of PAH/soot concentrations with the relevant Stokes number; however, it was not possible to collapse the responses of different species into a single line. This was expected since only diffusion-limited responses were taken into account in the relevant discussions based on and in the very definition of the Stokes number, while the effects of slow PAH/soot chemistry were excluded. In fact, this point was considered by Rodrigues et al. [630] and it was shown analytically (through 
linearized asymptotic analysis) that the phase lag and the reduction of response amplitudes of a certain flame quantity (i.e., species concentrations) with the increase in frequency, are directly affected by the Damköhler number of the corresponding flame quantity. In addition, the authors [630] also demonstrated that the steady response of any flame property to strain rate also affects its responses (i.e., amplitude damping) under unsteady conditions. It can be generalized that flame responses to strain rate oscillation are primarily determined by: 1) the Stokes number, which compares the characteristic diffusion time scale with the imposed oscillation time scale; 2) the Damköhler number, which indicates the characteristic reaction time scale of a certain quantify of interests; and 3) a parameter that represents the steady response of a certain quantity of interests to strain rate variations [630].

\subsection{Various other effects}

Noteworthy counterflow-based soot studies for various other effects are discussed next.

\subsubsection{Soot formation in counterflow flames of fuel sprays}

In addition to use in the study of flames with gas-phase or pre-vaporized fuels, counterflow burners have also been employed in spray combustion studies $[813,814]$. Spray flame is a highly complex multi-phase phenomenon and much of its fundamental physics are still not fully understood. As such, experimental [815-817] and numerical [818-821] investigations of spray flames established in a simple flow field are useful for clarifying their major features. Previous studies have shown that droplet mean size, size distributions, and droplet evaporation rates are important factors that affect the general characteristics of spray flames, such as flame temperature and species concentration distributions [822-825]. Depending on the boundary conditions, both premixed-like and diffusionlike flames can exist in the combustion of fuel droplets [826]. Drop group combustion (i.e., the combustion of droplet clusters) was observed to behave like diffusion flames [827-829]. 
With respect to soot formation in spray counterflow flames, Chen and Gomez [816] studied two CDFs of quasi-monodispersed n-heptane sprays with the same global fuel/air ratios. The first flame existed as a thin blue sheet and was observed to behave similarly to that of a purely gaseous flame, which was rationalized by the fact that the droplets are completely evaporated before reaching the flame zone. In the second flame, however, with a higher initial mean droplet size and velocity, some droplets survived and penetrated the first flame of fuel vapors towards the oxygen-rich environment. As a result, a second flame, characterized by a thick orange region on the oxidizer side, was formed. The authors attributed this orange luminosity to radiation of small soot particles, although quantification of soot concentrations was not performed. Nevertheless, this study indicated that an increase in initial droplet size may enhance soot formation, a topic for which Hayashi et al. [603] conducted detailed experimental and numerical investigations. The authors established n-decane (n$\mathrm{C}_{10} \mathrm{H}_{22}$ ) spray flame in a laminar counterflow field, with a burner setup and flame pictures shown in Figure 48 [603]. A vibratory orifice atomizer (VOA), installed at the top of the upper (fuel) burner, provided quasi-monodispersed fuel droplets with variable mean droplet sizes. The fuel spray was premixed by air at $\phi=0.106$ and issued from the upper nozzle against stretch-stabilized lean methaneair premixed flames, mimicking the conditions in practical combustors where premixed fuel sprays approach the high temperature zone. A strong effect of initial droplet size on soot formation was observed. As the initial Sauter mean diameter (SMD) of the fuel droplets increased, an initially blue reaction zone transitioned towards yellow flames with increasingly higher luminosity, indicating an enhancement of both the peak soot concentrations and the sooting zone areas (further confirmed by corresponding LII measurements). In addition, an interesting local inhibition of soot formation was observed for larger SMD conditions, resulting in an annular shape for the spatial distribution of soot, with particle sizes being larger in the radially outer region of the soot zone. This local inhibition can be attributed to the evaporative cooling effects caused by group combustion of the unburned, initially large fuel droplets. 


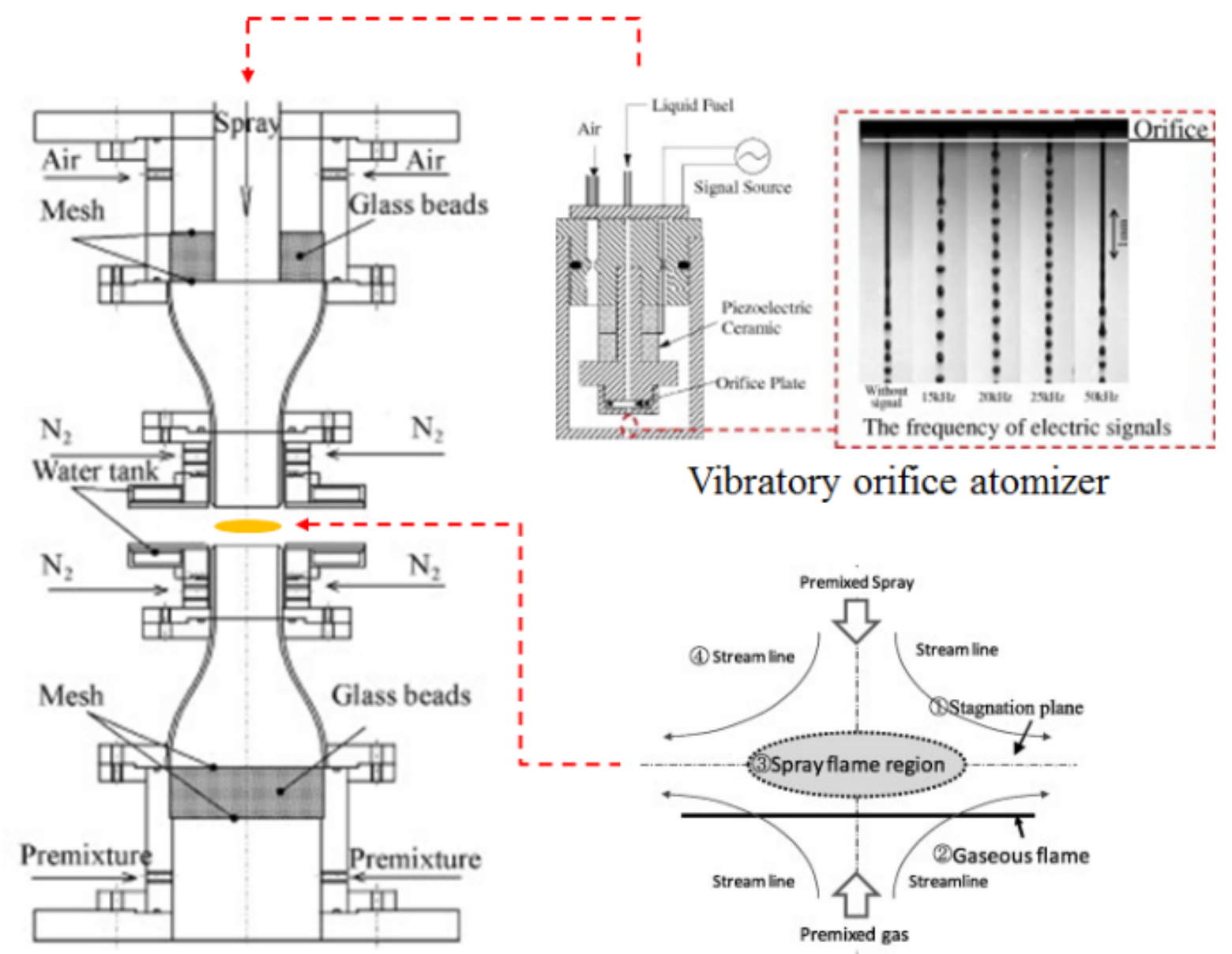

Figure 48. Experimental setup for the investigation of counterflow flames of fuel sprays employed by Hayashi et al [603]. Shown are the counterflow burner with two opposing converging nozzles, the vibratory orifice atomizer attached to the upper burner for the generation of liquid sprays with controllable droplet sizes, and a schematic of the spray CDF. Reprinted from Hayashi et al [603] with permission of Elsevier.

Droplet size distribution can also significantly affect soot formation. Experimental results showed that both the soot concentration and the sooting zone area were reduced in spray counterflow flames for poly-dispersed droplets, as compared to quasi-mono dispersed droplets with the same SMD [612]. In poly-dispersed cases there were a larger number of small fuel droplets, more likely to be completely evaporated before reaching the high temperature flame zone. As a result, the premixed-like combustion mode was promoted, which is helpful for soot reduction. 


\subsubsection{Effects of electric fields on soot formation in CDFs}

Hydrocarbon flames are abundant with electrically charged species [830] such as the $\mathrm{H}_{3} \mathrm{O}^{+}, \mathrm{CHO}^{+}$, $\mathrm{CH}_{3}{ }^{+}$and $\mathrm{C}_{3} \mathrm{H}_{3}{ }^{+}$ions [831, 832], ions of PAHs [833], and carbon clusters in the form of $\mathrm{C}_{2 n}$ with 30 $\leq 2 \mathrm{n} \leq 210$ (both positive and negative) [834, 835], in addition to charged soot particles [133, 836, 837]. It is therefore reasonable to expect the flame to be significantly affected when exposed to external electric fields. Indeed, it has been shown that electric field-assisted combustion can change flame shapes $[838,839]$ and temperatures [840], improve flame instability [841-843], enhance flame propagation speeds [844-846], and affect soot emissions [527, 566, 847-850].

Whether soot formation in a certain CDF can be inhibited depends on the intensity, polarity (for DC), and frequency (for AC) of the applied electric fields [527]. In sooting ethylene CDFs, where the fuel nozzle is connected to the high-voltage terminal and the oxidizer nozzle is grounded, a small positive voltage did not notably affect the flame position or soot formation, but larger voltages resulted in flame instability. However, applying negative voltage $<-2 \mathrm{kV}$ reduced soot/PAH concentrations significantly in both SF and SFO flame configurations. The measured spatial distributions of OH / PAH LIF, and soot LII signals, are shown in Figure 49 for both the SF (a) / SFO (b) baseline flames and those with a negative voltage of $-2.0 \mathrm{kV}$ applied (c, d). The locations of the OH LIF zones were seen to be dragged towards the fuel side by the electric field, consistent with the direction of ionic wind [851, 852], since there are more positive ions than negative. Similarly, the stagnation plane was also forced closer to the fuel side. Since soot is always formed on the fuel side of the flame, these movements of the flame front and stagnation plane are expected to result in a narrower fuel pyrolysis zone and less residence time for PAH/soot growth. Furthermore, heat loss toward the burner nozzle may also be enhanced, leading to lower flame temperature and reduced soot formation rates. Note that these explanations are based solely on the effects of ionic wind. Although reasonable, they do not explain the flame instability when positive potential is applied, or the fact that 
in certain flames [850] luminosity was always seen to shift away from the fuel nozzle, independent of the polarity of the DC potential.
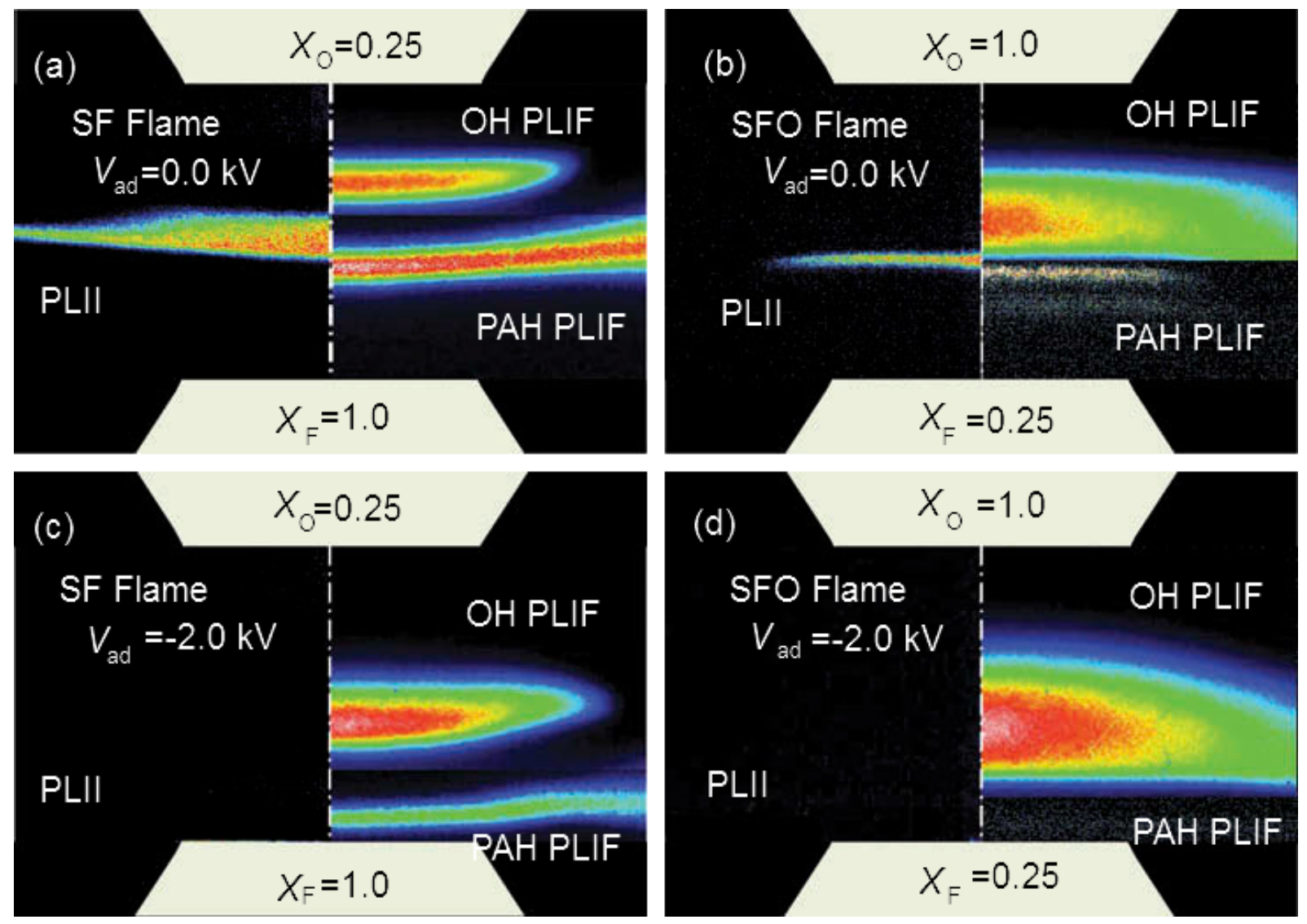

Figure 49. Effects of DC electric field on measured spatial distributions of OH / PAH LIF and soot LII signals. Reprinted from Park et al. [527] with permission of Taylor \& Francis.

Kono and coworkers [850] applied AC potentials in sooting $\mathrm{C}_{2} \mathrm{H}_{2}$ CDFs. At an AC frequency of $327 \mathrm{kHz}$, the authors observed that SVF profiles shifted away from the fuel nozzle and peak SVF increased when the peak-to-peak voltage was $4.8 \mathrm{kV}$, while the opposite was true when it was $1.6 \mathrm{kV}$. These complex behaviors were thought to result from forces other than the ionic wind effects. Clearly, more mechanistic investigations are required to explain these experimental observations.

\subsubsection{Effects of soot radiation on flame characteristics}

CDF is a classic tool for investigating critical strain rate in diffusion flame extinction $[109,853]$, which are routinely used as an important global flame property for the validation of detailed kinetic 
mechanisms (see e.g., Refs. [587, 854-856]). CDF can be extinguished at both high and low strain rates [857-860]. Available time for mixing is short at sufficiently high strain rates, and the flame is extinguished due to insufficient fuel/oxidizer mixing. At very low strain rates, flame intensity is low and relative heat loss due to radiation becomes critically important, which can notably decrease flame temperature (increase reaction time) and finally, extinguish the flame.

Heat loss due to radiation from soot particles can be significant, especially when considering the multi-dimensional effect in practical flames where soot produced in fuel-rich regions can be transported (by convection and/or turbulence) to the high-temperature reaction zone to exert influence on flammability. To analyze the effects of radiation heat loss from this non-local soot on the flame extinction limit, an analytical study, using large activation energy asymptotic theory, was performed for $\mathrm{C}_{2} \mathrm{H}_{4} \mathrm{CDF}$, with pre-loaded soot particles in the oxidizer stream [604]. The results, shown in Figure 50, demonstrate that the presence of soot had a strong effect in reducing the size of the flammable domain. Particularly, with an initial soot mass fraction of $5 \%$, the lower strain rate radiation-extinction limit would be increased by more than one magnitude, and the upper mixinglimited extinction strain rate could be decreased by around $30 \%$.

In vigorously burning CDFs, the presence of flame-generated soot particles also tends to reduce soot formation as a result of radiative cooling of the flame [583,613,627, 861]. The extent of such reduction is higher in flames with a lower strain rate [627]. Nevertheless, in typical SF flames, the effect of soot radiation was found to be weaker than that of gas radiation [583]. This is because in SF flames, SVF peaks at the stagnation plane, which is spatially separated from the high-temperature reaction zone and thus has a relatively low temperature of around $1000 \mathrm{~K}$. On the other hand, the most effective gaseous radiators such as $\mathrm{CO}_{2}$ and $\mathrm{H}_{2} \mathrm{O}$ peak near the reaction zone with much higher local temperature. For the same reason, soot radiation in SF flames may be less important than in coflow flames, where SVF peaks in regions of relatively higher temperatures. 


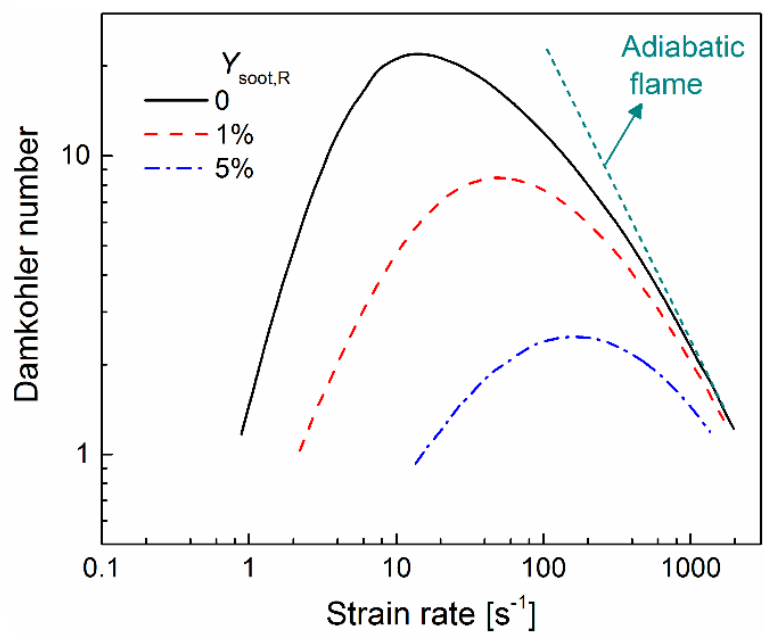

Figure 50. Effects of soot loading on extinction strain rate in counterflow diffusion flames, numerical data taken from Narayanan et al. [604]. For non-adiabatic flame, extinction can be seen to occur at both high and low strain rates due to an excessively small Damköhler number; and the presence of soot particles noticeably affects the lower critical strain rate for extinction.

\subsubsection{Interactions between soot and nitric oxides}

The formation of nitrogen oxides $(\mathrm{NOx})$ in hydrocarbon-air flames is known to be temperature sensitive and can therefore be influenced by radiative heat loss from gaseous species and soot particles, if present $[862,863]$. In addition, oxidation of soot particles and precursors may compete with thermal $\mathrm{NOx}$ formation for $\mathrm{O}$ and $\mathrm{OH}$ radicals, contributing to the reduction of $\mathrm{NOx}$ emission [539]. On the other hand, nitric dioxide $\left(\mathrm{NO}_{2}\right)$, as a form of $\mathrm{NOx}$, is a strong soot oxidizing agent-even more effective than $\mathrm{O}_{2}[327,330]$. In this regard, soot and $\mathrm{NOx}$-being the two most worrying pollutants from hydrocarbon fuel combustion-can interact in their formation processes.

A dedicated numerical analysis was performed to investigate soot and NO interactions in ethylene CDFs of the SF type [864]. The model included a detailed reaction mechanism with NOx chemistry, a two-equation semi-empirical $\mathrm{C}_{2} \mathrm{H}_{2}$-based soot sub-model [376], and radiation heat loss from major gaseous species and soot particles. Three simulation runs were conducted to investigate the detailed mechanisms that determine how the presence of soot affects NOx formation. In the first run (SIM1), both NOx and soot models were included, and in the third run (SIM3) only the NOx model was 
retained. The second run (SIM2) was computed with a fixed temperature, obtained from SIM1, while all rates related to soot were set to zero. As a result, the difference of the predicted NOx concentrations between SIM2 and SIM3 was caused by purely thermal effects from soot radiation. The differences between SIM1 and SIM2 were due to the chemical effect of soot formation, as the production of soot is expected to alter the chemical structures of the flames by-for example-consuming gaseous soot precursors. Both thermal and chemical effects of soot on NO formation were observed, as can be seen in Figure 51. In terms of the chemical effect, analysis of the detailed reaction pathway suggests that the most important competing species between soot and NOx chemistry is $\mathrm{C}_{2} \mathrm{H}_{2}$.

On the other hand, comparison between two numerical runs with and without the NOx subchemistry shows that the formation of NOx has a negligible effect on soot formation/oxidation in the flames investigated. However, it should be noted that the authors [864] only considered the effect of $\mathrm{NO}$, and further investigations taking $\mathrm{NO}_{2}$ formation into account may be needed. In addition, studies on more flame configurations, like SFO CDFs, are necessary to confirm whether the insignificance of NOx on soot formation can be generalized. This is especially relevant considering that in SF CDF, the peak locations of NOx and soot are, in fact, spatially separated.

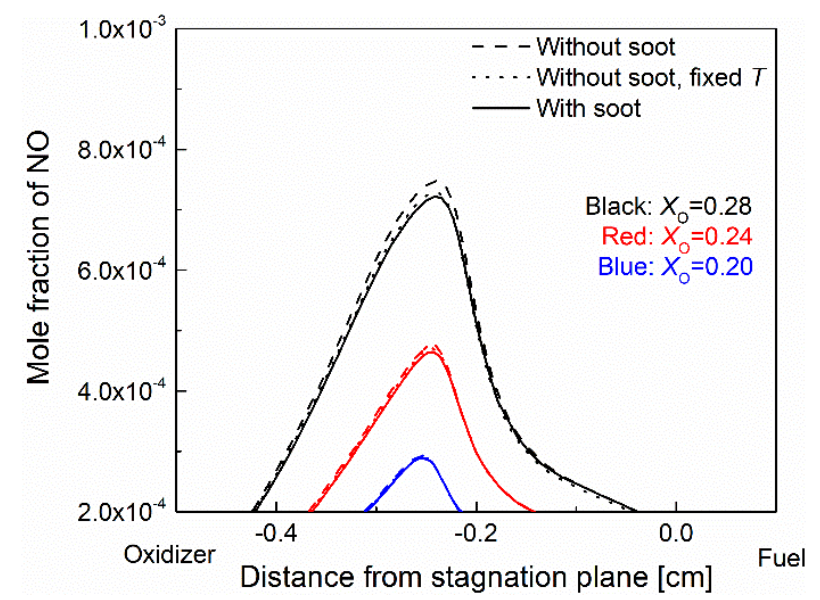

Figure 51. Effects of soot formation on NO formation in CDFs. Data taken from Guo et al. [864]. 


\section{Summary, challenges, and prospects}

In view of the growing global energy demand, conventional hydrocarbon fuel is expected to continue as the dominant energy source in the foreseeable future. However, the consensus is that hazardous emissions from the combustion of hydrocarbon fuels must be mitigated for a sustainable future. As a ubiquitous combustion phenomenon, soot formation has attracted continuous research attention, not only because of its associated negative environmental impact, but for the rich physicochemical processes involved which are still not fully understood. Nevertheless, the outcome from decades of fundamental research-especially those performed in well-controlled laboratory-scale flames-confirms that it is possible to describe soot formation from first principles.

Although the primary objective of this review is to provide a comprehensive summary of soot research performed with counterflow flames, many relevant aspects involve fundamental soot mechanisms that are universally applicable and cannot be isolated from other research. Therefore, the concluding remarks that follow list several general challenges and opportunities for soot research, before discussion of those more specific to counterflow flames.

As discussed extensively in the text, the conceptual stages of soot formation include 1) gas phase molecular growth towards large PAHs; 2) soot nucleation out of the gas phase; 3) surface growth and particle-particle coagulations; and 4) soot oxidation and fragmentation. Despite research progress made thus far, there are still unresolved aspects in each of these processes that continue to challenge the soot research community. The most prominent challenges (in our opinion) are as follows:

- Molecular growth from aliphatic fuels involves the formation of the first aromatic ring, in most cases, benzene. Although there are various reaction pathways for benzene formation, and the relative importance of each pathway may depend on fuel molecular structure, the number of pathways is finite, and fairly detailed knowledge is available about many of them [172]. However, the story is far different for molecular growth beyond benzene. As discussed in section 2.1.2, new pathways for PAH growth are being identified (and proposed) continuously. 
But experimental validation of these pathways becomes challenging since a) the concentration of large PAHs are typically too low for accurate measurement; and b) experimental techniques to resolve the many isomers of large PAHs are limited. In fact, in view of the formidable number of isomers for molecules with increasingly larger sizes, the question remains whether all the relevant pathways [865] can ever be captured. A relevant question is to what details would we need for practical purposes.

- The transition from gas-phase molecular soot precursors to condensed-phase particles has remained a mystery for many decades. The assumption of physical dimerization of PAHs has been implemented in many soot models, successfully explaining many experimental observations. However, theoretical analysis determined that at flame temperatures, PAHs with enough abundances are too volatile to be physically linked. As such, it is believed that covalent interactions are needed to bind the PAHs to form molecular clusters (incipient particles). While previous mechanisms for covalent cluster formation required repeated high energy barrier activation of stable PAHs (and thus not perfectly compatible with the rapid nucleation of soot, even in low temperature radical-starved regions), the recently proposed clustering of hydrocarbon by radical chain reaction (CHRCR) mechanism [138] highlights the role of radical chain propagation through resonantly stabilized radicals. This new CHRCR mechanism overcomes the issue of low molecular growth rates associated with existing chemical nucleation mechanisms and also allows $\sigma$-dimerization from large RSRs [138, 273]. Although promising, one challenge is that detailed reaction rates must be established for the proposed reactions so that an extensive kinetic mechanism can be assembled to test whether CHRCR can explain experimental features on soot formation. The target for testing may include, for example, the bimodal size distribution in the post-flame zone of a premixed stagnation flame, the non-monotonic variations of soot formation with temperature in a shock tube and premixed flames, the synergistic effects between fuels on soot formation in diffusion flames and many 
more. Furthermore, the role of charged ions in soot nucleation must be explored further; it is possible that different mechanisms can simultaneously contribute to soot nucleation.

- Reactions that occur on the surface of soot particles account for most of soot mass growth, so a deeper understanding of these surface reactions is critical in order to predict the amount of soot formed in flames and practical combustion devices. In most current soot models, surface reaction is dominated by the surface-HACA mechanism, in which $\mathrm{C}_{2} \mathrm{H}_{2}$ is the most important mass growth agent. Rates for heterogeneous hydrogen abstraction and $\mathrm{C}_{2} \mathrm{H}_{2}$ addition reactions are obtained by assuming a kinetic analogy between soot and gas phase aromatic species. Currently, however, it is unclear how and to what extent surface morphology, adsorption/desorption and other features specific to heterogeneous reactions will affect these surface reactions [865]. The effects of surface properties (e.g., graphitization) on soot growth are yet to be studied quantitatively. To date, there is no fundamental models that describe how the surface properties change as the soot particle evolves in the flame. Such lack of detailed knowledge about surface reactions is reflected in the wide variability of the parameter $\alpha$ (representing the fraction of surface site that is active for surface reactions) in different soot models. It is a common practice to adjust $\alpha$ to match experimental soot concentrations. In addition to $\mathrm{C}_{2} \mathrm{H}_{2}$, the recent CHRCR mechanism [138] also suggests that surface growth can proceed with radical-chain reactions through RSRs. The possibility that aliphatic chains can be present at the surface of nascent particles adds more complexity to a complete description of surface reactions. It is obvious that more fundamental research is required to gain further insights into the heterogeneous reactions on soot surfaces.

- Soot oxidation is essential in determining ultimate soot production, and in the regeneration of particulate filters, which are now routinely used to control soot emission from IC enginepowered ground vehicles. The oxidation of soot involves complex heterogeneous surface reactions; therefore, many of the challenges associated with soot surface growth discussed 
above also apply to soot oxidation. In addition, experimental evidence of the particle internal burning mode [348] may also suggest the importance of surface diffusion of oxidizing agents. However, many current models for soot oxidation are still largely empirical in that they typically take bulk carbon as the sole solid reactant, neglecting any steric effects and the many different kinetic features of soot formed in different environments. Intermediate products of soot oxidation also require further research attention as it was shown in SFO flames, the oxidation of soot may produce a second peak for PAH LIF signals, indicating the complexity of soot oxidation products. It is clear that future research is needed to uncover more detailed and sterically-resolved information on how oxidizing species such as $\mathrm{O}, \mathrm{OH}, \mathrm{O}_{2}$ and $\mathrm{NO}_{2}$ interact with soot particles for the removal of soot mass.

In addition to these challenges, soot formation is further complicated because of its dependence on many combustion parameters. The present review clearly demonstrates that research conducted in counterflow flames has notably contributed to our understanding of the effects of fuel molecular structure, fuel/oxidizer dilution, pressure, temperature, residence time, flame structure, and unsteadiness on soot formation. Further investigations of the underlying mechanisms of these phenomenological effects in turn provide hints through which various aspects of soot formation fundamentals can be uncovered. This review also identifies several challenges that must be addressed for more complete soot characterization in counterflow flames and for further contributions to a first principle understanding of soot. Following are some pertinent issues, along with prospects for future research:

- Characterization of soot in counterflow flames is typically limited to soot volume fraction, primary particle size, and total number densities, while more detailed information such as particle size distribution (PSD) is rarely reported. This is because conventional sampling methods are too intrusive for counterflow flames, which have a very narrow sooting zone $(\sim 1$ $\mathrm{mm})$. However, PSD can provide valuable information about nearly all aspects of soot 
information and it is essential if detailed insight is to be derived from counterflow flame experimental data. Future effort is needed to develop reliable, in-situ methods (e.g., timeresolved LII) to measure PSD in CDFs. Challenges also exist for the thermophoretic extraction of soot particles in counterflow flames, because the TEM probe used in traditional fastinsertion methods may vibrate too much to allow for soot extraction at a specific location. The SiC wire method developed by Figura and Gomez [508] may partly solve this problem.

- The narrow sooting zone in counterflow flames also indicates that complete soot evolution from gas-phase soot precursors (via nascent particles to mature soot particles) occurs in a somewhat confined spatial region, necessitating high spatial resolution experimental techniques. Conventional optical methods for quantitative soot measurements, such as light extinction and scattering, are dependent on the complex refractive index of soot, which is known to vary with particle size and maturity. However, a quantitative relationship is yet to be determined. Additional methods may be necessary (i.e., small angle X-ray or neutron scattering $[866,867])$ to confirm the extent to which the varying optical properties may affect the quantitative soot measurements in counterflow flames.

- There exist a number of counterflow flame-based studies regarding the synergistic effects of fuel mixtures on PAH and soot formation, demonstrating that certain binary fuel mixtures can have higher sooting tendency than either fuel component (e.g. [86, 528, 586]). These works highlight the importance of chemical interactions between different fuels and so have practical application for fuel formulation. While future opportunities exist for the identification and kinetic explanation of additional fuel pairs exhibiting synergistic effects, it is interesting to search fuel mixtures with lower sooting tendencies than either component, which exhibit a negatively synergistic effect.

- To be more relevant to practical combustion systems like diesel engines and jet engines, counterflow flame experiments must be extended to operate with liquid fuels at high-pressure 
conditions. Although the counterflow configuration is advantageous for controllable and stable flame operation at elevated pressures (i.e., not sensitive to buoyancy-driven instability, no heat loss through burner rim) [788], it is important to design boundary conditions carefully, so that pressure effects on soot formation can be isolated $[537,618,788]$. In terms of fuel types, studies on oxygenated fuels with aromatic rings are scarce, but they deserve attention in future research because of their practical uses and potentially different soot growth pathways, as compared to other aliphatic and aromatic fuels.

- Counterflow flames are particularly suitable for studying the effects of fuel-borne catalysts on soot formation and oxidation (FBCs, like those metal-containing compounds reviewed here). This is because the formation and oxidation of soot can be separated by utilizing either SF or SFO flames. The challenge is to develop effective measurement techniques that distinguish soot and catalytic particles so that concentrations of each can be reliably determined. Possibilities include methods based on spectral emissions [868, 869], but it should be considered that these methods need high spatial resolution for applications in counterflow flames.

- Time-resolved experimental data on PAH, and soot in CDFs subject to oscillating strain rates, are scarce $[585,801]$. Such data can provide not only information about the unsteady flamelet model, but it can validate kinetic models for PAH and soot formation. Peaks, troughs, and mean values of $\mathrm{PAH} /$ soot concentrations in oscillating flames, normalized by their steady-state values, provide valuable information regarding the kinetics of their formation.

\section{Acknowledgements}

The preparation of this manuscript was supported by the National Natural Science Foundation of China (51606136) and the Clean Combustion Research Center of KAUST. The authors are grateful to Drs. S. Mani Sarathy, Hong G. Im and William L. Roberts of KAUST, Dr. Abhijeet Raj of Khalifa University and Dr. Sungwoo Park of the Korea Aerospace University for collaboration on relevant 
projects. YW also acknowledged stimulating and informative discussions with Dr. Alessandro Gomez of Yale University, Dr. Dongping Chen of Beijing Institute of Technology and Dr. Xunchen Liu of Shanghai Jiaotong University. Technical assistances from Lei Xu, Wei Dai, Mengxiang Zhou and Wei Wang of Wuhan University of Technology in organizing some of the cited references are also acknowledged.

\section{References:}

[1] U.S. Energy Information Administration, International Energy Outlook; 2016.

[2] Reitz RD. Directions in internal combustion engine research. Combust Flame 2013;160:1-8.

[3] Leach FCP, Stone R, Richardson D, Turner JWG, Lewis A, Akehurst S, Remmert S, Campbell

$\mathrm{S}$, Cracknell R. The effect of oxygenate fuels on PN emissions from a highly boosted GDI engine. Fuel 2018;225:277-86.

[4] Zhao H. Overview of gasoline direct injection engines. In: Zhao H, editor. Advanced Direct Injection Combustion Engine Technologies and Development: Woodhead Publishing; 2010. p. 119.

[5] Kennedy IM. The health effects of combustion-generated aerosols. Proc Combust Inst 2007;31:2757-70.

[6] Oberdorster G, Sharp Z, Atudorei V, Elder A, Gelein R, Kreyling W, Cox C. Translocation of inhaled ultrafine particles to the brain. Inhal Toxicol 2004;16:437-45.

[7] Howard JB, Longwell JP, Marr JA, Pope CJ, Busby Jr WF, Lafleur AL, Taghizadeh K. Effects of PAH isomerizations on mutagenicity of combustion products. Combust Flame 1995;101:262-70.

[8] Schwartz J, Dockery DW. Increased mortality in Philadelphia associated with daily air pollution concentrations. Am Rev Respir Dis 1992;145:600-4.

[9] Schwartz J, Laden F, Zanobetti A. The concentration-response relation between $\operatorname{PM}(2.5)$ and daily deaths. Environ Health Persp 2002;110:1025-9.

[10] Bond TC, Doherty SJ, Fahey DW, Forster PM, Berntsen T, DeAngelo BJ, Flanner MG, Ghan S, Karcher B, Koch D, Kinne S, Kondo Y, Quinn PK, Sarofim MC, Schultz MG, Schulz M, Venkataraman C, Zhang H, Zhang S, Bellouin N, Guttikunda SK, Hopke PK, Jacobson MZ, Kaiser JW, Klimont Z, Lohmann U, Schwarz JP, Shindell D, Storelvmo T, Warren SG, Zender CS. Bounding the role of black carbon in the climate system: A scientific assessment. J Geophys Res Atmos 2013;118:5380-552.

[11] Hansen J, Nazarenko L. Soot climate forcing via snow and ice albedos. P Natl Acad Sci USA 2004;101:423-8.

[12] Horvath H. Atmospheric light absorption-A review. Atmos Environ A - Gen 1993;27:293317.

[13] Huang RJ, Zhang YL, Bozzetti C, Ho KF, Cao JJ, Han YM, Daellenbach KR, Slowik JG, Platt SM, Canonaco F, Zotter P, Wolf R, Pieber SM, Bruns EA, Crippa M, Ciarelli G, Piazzalunga A, Schwikowski M, Abbaszade G, Schnelle-Kreis J, Zimmermann R, An ZS, Szidat S, Baltensperger U, El Haddad I, Prevot ASH. High secondary aerosol contribution to particulate pollution during haze events in China. Nature 2014;514:218-22.

[14] Koch D, Balkanski Y, Bauer SE, Easter RC, Ferrachat S, Ghan SJ, Hoose C, Iversen T, Kirkevåg A, Kristjansson JE, Liu X, Lohmann U, Menon S, Quaas J, Schulz M, Seland Ø, Takemura T, Yan N. Soot microphysical effects on liquid clouds, a multi-model investigation. Atmos Chem Phys 2011;11:1051-64. 
[15] Ackerman AS, Toon OB, Stevens DE, Heymsfield AJ, Ramanathan V, Welton EJ. Reduction of tropical cloudiness by soot. Science 2000;288:1042-7.

[16] Hendricks J, Karcher B, Lohmann U, Ponater M. Do aircraft black carbon emissions affect cirrus clouds on the global scale? Geophys Res Lett 2005;32.

[17] Andreae MO, Gelencser A. Black carbon or brown carbon? The nature of light-absorbing carbonaceous aerosols. Atmos Chem Phys 2006;6:3131-48.

[18] Wang H. Formation of nascent soot and other condensed-phase materials in flames. Proc Combust Inst 2011;33:41-67.

[19] Musculus MPB, Pickett LA. Diagnostic considerations for optical laser-extinction measurements of soot in high-pressure transient combustion environments. Combust Flame 2005;141:371-91.

[20] Joo HI. Soot formation in non-premixed laminar flames at subcritical and supercritical pressures [Ph.D. thesis]: University of Toronto; 2010.

[21] Zhou L, Dam NJ, Boot MD, de Goey LPH. Investigation of the effect of molecular structure on sooting tendency in laminar diffusion flames at elevated pressure. Combust Flame 2014;161:266977.

[22] Eaves NA, Thomson MJ, Dworkin SB. The effect of conjugate heat transfer on soot formation modeling at elevated pressures. Combust Sci Technol 2013;185:1799-819.

[23] Iannuzzi SE, Barro C, Boulouchos K, Burger J. Combustion behavior and soot formation/oxidation of oxygenated fuels in a cylindrical constant volume chamber. Fuel 2016;167:49-59.

[24] Zhang J, Jing W, Roberts WL, Fang T. Soot measurements for diesel and biodiesel spray combustion under high temperature highly diluted ambient conditions. Fuel 2014;135:340-51. [25] Xiao MY, Liu HF, Bi XJ, Wang H, Lee CFF. Experimental and numerical investigation on soot behavior of soybean biodiesel under ambient oxygen dilution in conventional and lowtemperature flames. Energ Fuel 2014;28:2663-76.

[26] Qi DH, Chen B, Zhang D, Lee CF. Optical study on the combustion characteristics and soot emissions of diesel-soybean biodiesel-butanol blends in a constant volume chamber. J Energy Inst 2016;89:807-20.

[27] Jing W, Wu ZY, Roberts WL, Fang TG. Spray combustion of biomass-based renewable diesel fuel using multiple injection strategy in a constant volume combustion chamber. Fuel 2016;181:718-28.

[28] Ryser R, Gerber T, Dreier T. Soot particle sizing during high-pressure Diesel spray combustion via time-resolved laser-induced incandescence. Combust Flame 2009;156:120-9.

[29] Liu HF, Lee CF, Huo M, Yao MF. Comparison of ethanol and butanol as additives in soybean biodiesel using a constant volume combustion chamber. Energ Fuel 2011;25:1837-46.

[30] Xuan T, Desantes JM, Pastor JV, Garcia-Oliver JM. Soot temperature characterization of spray a flames by combined extinction and radiation methodology. Combust Flame 2019;204:290-303.

[31] Eremin AV. Formation of carbon nanoparticles from the gas phase in shock wave pyrolysis processes. Prog Energy Combust Sci 2012;38:1-40.

[32] Hong Z, Davidson DF, Vasu SS, Hanson RK. The effect of oxygenates on soot formation in rich heptane mixtures: A shock tube study. Fuel 2009;88:1901-6.

[33] Alexiou A, Williams A. Soot formation in shock-tube pyrolysis of toluene, toluene-methanol, toluene-ethanol, and toluene-oxygen mixtures. Combust Flame 1996;104:51-65.

[34] Kern RD, Xie K. Shock tube studies of gas phase reactions preceding the soot formation process. Prog Energy Combust Sci 1991;17:191-210.

[35] Frenklach M, Yuan T. Effect of alcohol addition on shock-initiated formation of soot from benzene. Proceedings of the 16th International Symposium on Shock Tubes and Waves 1987. p. 487-93. 
[36] Frenklach M, Clary DW, Gardiner Jr WC, Stein SE. Effect of fuel structure on pathways to soot. Proc Combust Inst 1988;21:1067-76.

[37] Mathieu O, Chaumeix N, Paillard CE. Soot formation from a distillation cut of a FischerTropsch diesel fuel: A shock tube study. Combust Flame 2012;159:2192-201.

[38] Agafonov GL, Smirnov VN, Vlasov PA. Shock tube and modeling study of soot formation during the pyrolysis and oxidation of a number of aliphatic and aromatic hydrocarbons. Proc Combust Inst 2011;33:625-32.

[39] Kc U, Beshir M, Farooq A. Simultaneous measurements of acetylene and soot during the pyrolysis of ethylene and benzene in a shock tube. Proc Combust Inst 2017;36:833-40.

[40] Eremin A, Gurentsov E, Mikheyeva E. Experimental study of temperature influence on carbon particle formation in shock wave pyrolysis of benzene and benzene-ethanol mixtures. Combust Flame 2015;162:207-15.

[41] Litzinger T, Colket M, Kahandawala M, Katta V, Lee SY, Liscinsky D, McNesby K, Pawlik R, Roquemore M, Santoro R, Sidhu S, Stouffer S, Wu J. Fuel additive effects on soot across a suite of laboratory devices, Part 1: Ethanol. Combust Sci Technol 2009;181:310-28.

[42] Lam FW, Howard JB, Longwell JP. The behavior of polycyclic aromatic hydrocarbons during the early stages of soot formation. Proc Combust Inst 1989;22:323-32.

[43] Rezgui Y, Guemini M. Effect of ethanol addition on soot precursors emissions during benzene oxidation in a jet-stirred reactor. Environ Sci Pollut R 2014;21:6671-86.

[44] Litzinger T, Colket M, Kahandawala M, Lee SY, Liscinsky D, McNesby K, Pawlik R, Roquemore M, Santoro R, Sidhu S, Stouffer S. Fuel additive effects on soot across a suite of laboratory devices, Part 2: Nitroalkanes. Combust Sci Technol 2011;183:739-54.

[45] Babushok VI, Tsang W, McNesby KL. Additive influence on polycyclic aromatic hydrocarbon formation. Proc Combust Inst 2002;29:2315-23.

[46] Saggese C, Ferrario S, Camacho J, Cuoci A, Frassoldati A, Ranzi E, Wang H, Faravelli T. Kinetic modeling of particle size distribution of soot in a premixed burner-stabilized stagnation ethylene flame. Combust Flame 2015;162:3356-69.

[47] Abid AD, Camacho J, Sheen DA, Wang H. Evolution of soot particle size distribution function in burner-stabilized stagnation n-dodecane-oxygen-argon flames. Energ Fuel 2009;23:4286-94.

[48] Abid AD, Camacho J, Sheen DA, Wang H. Quantitative measurement of soot particle size distribution in premixed flames - The burner-stabilized stagnation flame approach. Combust Flame 2009; 156:1862-70.

[49] Camacho J, Liu C, Gu C, Lin H, Huang Z, Tang Q, You X, Saggese C, Li Y, Jung H, Deng L, Wlokas I, Wang H. Mobility size and mass of nascent soot particles in a benchmark premixed ethylene flame. Combust Flame 2015;162:3810-22.

[50] Yapp EKY, Chen D, Akroyd J, Mosbach S, Kraft M, Camacho J, Wang H. Numerical simulation and parametric sensitivity study of particle size distributions in a burner-stabilised stagnation flame. Combust Flame 2015;162:2569-81.

[51] Sirignano M, Bartos D, Conturso M, Dunn M, D'Anna A, Masri AR. Detection of nanostructures and soot in laminar premixed flames. Combust Flame 2017;176:299-308.

[52] Conturso M, Sirignano M, D'Anna A. Effect of 2,5-dimethylfuran doping on particle size distributions measured in premixed ethylene/air flames. Proc Combust Inst 2017;36:985-92.

[53] Carbone F, Gleason K, Gomez A. Probing gas-to-particle transition in a moderately sooting atmospheric pressure ethylene/air laminar premixed flame. Part I: Gas phase and soot ensemble characterization. Combust Flame 2017;181:315-28.

[54] Carbone F, Moslih S, Gomez A. Probing gas-to-particle transition in a moderately sooting atmospheric pressure ethylene/air laminar premixed flame. Part II: Molecular clusters and nascent soot particle size distributions. Combust Flame 2017;181:329-41. 
[55] Desgroux P, Faccinetto A, Mercier X, Mouton T, Aubagnac Karkar D, El Bakali A. Comparative study of the soot formation process in a "nucleation" and a "sooting" low pressure premixed methane flame. Combust Flame 2017;184:153-66.

[56] Smooke MD, Mcenally CS, Pfefferle LD, Hall RJ, Colket MB. Computational and experimental study of soot formation in a coflow, laminar diffusion flame. Combust Flame 1999;117:117-39.

[57] Santoro RJ, Semerjian HG, Dobbins RA. Soot particle measurements in diffusion flames. Combust Flame 1983;51:203-18.

[58] Legros G, Wang QL, Bonnety J, Kashif M, Morin C, Consalvi JL, Liu FS. Simultaneous soot temperature and volume fraction measurements in axis-symmetric flames by a two-dimensional modulated absorption/emission technique. Combust Flame 2015;162:2705-19.

[59] Smooke MD, Long MB, Connelly BC, Colket MB, Hall RJ. Soot formation in laminar diffusion flames. Combust Flame 2005;143:613-28.

[60] Gülder ÖL, Thomson KA, Snelling DR. Effect of fuel nozzle material properties on soot formation and temperature field in coflow laminar diffusion flames. Combust Flame 2006;144:42633.

[61] Gülder ÖL, Intasopa G, Joo HI, Mandatori PM, Bento DS, Vaillancourt ME. Unified behaviour of maximum soot yields of methane, ethane and propane laminar diffusion flames at high pressures. Combust Flame 2011;158:2037-44.

[62] Kholghy M, Saffaripour M, Yip C, Thomson MJ. The evolution of soot morphology in a laminar coflow diffusion flame of a surrogate for Jet A-1. Combust Flame 2013;160:2119-30. [63] Slavinskaya NA, Riedel U, Dworkin SB, Thomson MJ. Detailed numerical modeling of PAH formation and growth in non-premixed ethylene and ethane flames. Combust Flame 2012;159:97995.

[64] Kashif M, Bonnety J, Matynia A, Da Costa P, Legros G. Sooting propensities of some gasoline surrogate fuels: Combined effects of fuel blending and air vitiation. Combust Flame 2015;162:1840-7.

[65] Gomez A, Littman MG, Glassman I. Comparative study of soot formation on the centerline of axisymmetrical laminar diffusion flames - Fuel and temperature effects. Combust Flame 1987;70:225-41.

[66] Gomez A, Glassman I. Quantitative comparison of fuel soot formation rates in laminar diffusion flames. Proc Combust Inst 1988;21:1087-95.

[67] Das DD, McEnally CS, Pfefferle LD. Sooting tendencies of unsaturated esters in nonpremixed flames. Combust Flame 2015;162:1489-97.

[68] McEnally CS, Pfefferle LD. Experimental study of nonfuel hydrocarbons and soot in coflowing partially premixed ethylene/air flames. Combust Flame 2000;121:575-92.

[69] Steinmetz SA, Fang T, Roberts WL. Soot particle size measurements in ethylene diffusion flames at elevated pressures. Combust Flame 2016;169:85-93.

[70] Leschowski M, Thomson KA, Snelling DR, Schulz C, Smallwood GJ. Combination of LII and extinction measurements for determination of soot volume fraction and estimation of soot maturity in non-premixed laminar flames. Appl Phys B-Lasers and Optics 2015;119:685-96.

[71] Liu F, Dworkin SB, Thomson MJ, Smallwood GJ. Modeling DME addition effects to fuel on $\mathrm{PAH}$ and soot in laminar coflow ethylene/air diffusion flames using two PAH mechanisms.

Combust Sci Technol 2012;184:966-79.

[72] Guo HS, Gu ZZ, Thomson KA, Smallwood GJ, Baksh FF. Soot formation in a laminar ethylene/air diffusion flame at pressures from 1 to 8 atm. Proc Combust Inst 2013;34:1795-802. [73] Kang KT, Hwang JY, Chung SH, Lee W. Soot zone structure and sooting limit in diffusion flames: Comparison of counterflow and co-flow flames. Combust Flame 1997;109:266-81.

[74] Axelbaum RL, Flower WL, Law CK. Dilution and temperature effects of inert addition on soot formation in counterflow diffusion flames. Combust Sci Technol 1988;61:51-73. 
[75] Leusden CP, Peters N. Experimental and numerical analysis of the influence of oxygen on soot formation in laminar counterflow flames of acetylene. Proc Combust Inst 2000;28:2619-25.

[76] Du J, Axelbaum RL. The effect of flame structure on soot particle inception in diffusion flames. Combust Flame 1995;100:367-75.

[77] Xia F, Yablonsky GS, Axelbaum RL. Numerical study of flame structure and soot inception interpreted in carbon-to-oxygen atom ratio space. Proc Combust Inst 2013;34:1085-91.

[78] Wang H, Du DX, Sung CJ, Law CK. Experiments and numerical simulation on soot formation in opposed-jet ethylene diffusion flames. Proc Combust Inst 1996:2359-68.

[79] Gomez A, Rosner DE. Thermophoretic effects on particles in counterflow laminar diffusion flames. Combust Sci Technol 1993;89:335-62.

[80] Jahangirian S, McEnally CS, Gomez A. Experimental study of ethylene counterflow diffusion flames perturbed by trace amounts of jet fuel and jet fuel surrogates under incipiently sooting conditions. Combust Flame 2009;156:1799-809.

[81] Carbone F, Cattaneo F, Gomez A. Structure of incipiently sooting partially premixed ethylene counterflow flames. Combust Flame 2015;162:4138-48.

[82] Feng Q, Jalali A, Fincham AM, Wang YL, Tsotsis TT, Egolfopoulos FN. Soot formation in flames of model biodiesel fuels. Combust Flame 2012;159:1876-93.

[83] Deng SL, Koch JA, Mueller ME, Law CK. Sooting limits of nonpremixed n-heptane, nbutanol, and methyl butanoate flames: Experimental determination and mechanistic analysis. Fuel 2014;136:122-9.

[84] Singh P, Hui X, Sung C-J. Soot formation in non-premixed counterflow flames of butane and butanol isomers. Combust Flame 2016;164:167-82.

[85] Sung CJ, Li B, Wang H, Law CK. Structure and sooting limits in counterflow methane/air and propane/air diffusion flames from 1 to 5 atmospheres. Symp (Int) Combust 1998:1523-9.

[86] Karatas AE, Commodo M, Gülder ÖL. Soot formation in co- and counter-flow laminar diffusion flames of binary mixtures of ethylene and butane isomers and synergistic effects. Energ Fuel 2010;24:4912-8.

[87] Violi A, D'Anna A, D'Alessio A, Sarofim AF. Modeling aerosol formation in opposed-flow diffusion flames. Chemosphere 2003;51:1047-54.

[88] Conturso M, Sirignano M, D'Anna A. Effect of $\mathrm{C}_{9} \mathrm{H}_{12}$ alkylbenzenes on particle formation in diffusion flames: An experimental study. Fuel 2017;191:204-11.

[89] Sirignano M, Collina A, Commodo M, Minutolo P, D'Anna A. Detection of aromatic hydrocarbons and incipient particles in an opposed-flow flame of ethylene by spectral and timeresolved laser induced emission spectroscopy. Combust Flame 2012;159:1663-9.

[90] Sirignano M, Salamanca M, D'Anna A. The role of dimethyl ether as substituent to ethylene on particulate formation in premixed and counter-flow diffusion flames. Fuel 2014;126:256-62.

[91] Wang Y, Chung SH. Strain rate effect on sooting characteristics in laminar counterflow diffusion flames. Combust Flame 2016;165:433-44.

[92] Huijnen V, Evlampiev AV, Somers LMT, Baert RSG, de Goey LPH. The effect of the strain rate on $\mathrm{PAH} /$ soot formation in laminar counterflow diffusion flames. Combust Sci Technol 2010;182:103-23.

[93] Henríquez R, Demarco R, Consalvi JL, Liu F, Fuentes A. The oxygen index on soot production in propane diffusion flames. Combust Sci Technol 2014;186:504-17.

[94] Vandsburger U, Kennedy I, Glassman I. Sooting counterflow diffusion flames with varying oxygen index. Combust Sci Technol 1984;39:263-85.

[95] Gülder ÖL, Snelling DR. Influence of Nitrogen Dilution and Flame Temperature on Soot Formation in Diffusion Flames. Combust Flame 1993;92:115-24.

[96] Glassman I, Yaccarino P. The temperature effect in sooting diffusion flames. Proc Combust Inst 1981;18:1175-83. 
[97] Abid AD, Heinz N, Tolmachoff ED, Phares DJ, Campbell CS, Wang H. On evolution of particle size distribution functions of incipient soot in premixed ethylene-oxygen-argon flames. Combust Flame 2008;154:775-88.

[98] Harris MM, King GB, Laurendeau NM. Influence of Temperature and Hydroxyl Concentration on Incipient Soot Formation in Premixed Flames. Combust Flame 1986;64:99-112.

[99] Glassman I. Soot formation in combustion processes. Proc Combust Inst 1989;22:295-311. [100] Yoon SS, Anh DH, Chung SH. Synergistic effect of mixing dimethyl ether with methane, ethane, propane, and ethylene fuels on polycyclic aromatic hydrocarbon and soot formation. Combust Flame 2008;154:368-77.

[101] McNesby KL, Miziolek AW, Nguyen T, Delucia FC, Skaggs RR, Litzinger TA. Experimental and computational studies of oxidizer and fuel side addition of ethanol to opposed flow air/ethylene flames. Combust Flame 2005;142:413-27.

[102] Wu JT, Song KH, Litzinger T, Lee SY, Santoro R, Linevsky M. Reduction of PAH and soot in premixed ethylene-air flames by addition of dimethyl ether. Combust Sci Technol 2006;178:83763.

[103] Wu JT, Song KH, Litzinger T, Lee SY, Santoro R, Linevsky M, Colket M, Liscinsky D. Reduction of PAH and soot in premixed ethylene-air flames by addition of ethanol. Combust Flame 2006; 144:675-87.

[104] Yelverton TLB, Roberts WL. Effect of dilution, pressure, and velocity on smoke point in laminar jet flames. Combust Sci Technol 2008;180:1334-46.

[105] Peters N. Laminar flamelet concepts in turbulent combustion. Proc Combust Inst 1988;21:1231-50.

[106] Peters N. Laminar diffusion flamelet models in non-premixed turbulent combustion. Prog Energy Combust Sci 1984;10:319-39.

[107] Karataş AE, Gülder ÖL. Soot formation in high pressure laminar diffusion flames. Prog Energy Combust Sci 2012;38:818-45.

[108] Pitz RW, Hu S, Wang P. Tubular premixed and diffusion flames: Effect of stretch and curvature. Prog Energy Combust Sci 2014;42:1-34.

[109] Tsuji H. Counterflow Diffusion Flames. Prog Energy Combust Sci 1982;8:93-119.

[110] Eaves NA, Zhang Q, Liu F, Guo H, Dworkin SB, Thomson MJ. CoFlame: A refined and validated numerical algorithm for modeling sooting laminar coflow diffusion flames. Computer Physics Communications 2016;207:464-77.

[111] Slavinskaya NA, Frank P. A modelling study of aromatic soot precursors formation in laminar methane and ethene flames. Combust Flame 2009;156:1705-22.

[112] Chernov V, Thomson MJ, Dworkin SB, Slavinskaya NA, Riedel U. Soot formation with C1 and $\mathrm{C} 2$ fuels using an improved chemical mechanism for PAH growth. Combust Flame 2014;161:592-601.

[113] Hura HS, Glassman I. Fuel oxygen effects on soot formation in counterflow diffusion flames. Combust Sci Technol 1987;53:1-21.

[114] Böhm H, Kohse-Höinghaus K, Lacas F, Rolon C, Darabiha N, Candel S. On PAH formation in strained counterflow diffusion flames. Combust Flame 2001;124:127-36.

[115] Sunderland PB, Mortazavi S, Faeth GM, Urban DL. Laminar smoke points of nonbuoyant jet diffusion flames. Combust Flame 1994;96:97-103.

[116] Dotson KT, Sunderland PB, Yuan ZG, Urban DL. Laminar smoke points of coflowing flames in microgravity. Fire Safety J 2011;46:550-5.

[117] Berry TL, Roberts WL. Measurement of smoke point in velocity-matched coflow laminar diffusion flames with pure fuels at elevated pressures. Combust Flame 2006;145:571-8.

[118] Howard JB, Kausch Jr WJ. Soot control by fuel additives. Prog Energy Combust Sci 1980;6:263-76.

[119] Haynes BS, Wagner HG. Soot Formation. Prog Energy Combust Sci 1981;7:229-73. 
[120] Kennedy IM. Models of soot formation and oxidation. Prog Energy Combust Sci 1997;23:95132.

[121] Richter H, Howard JB. Formation of polycyclic aromatic hydrocarbons and their growth to soot - a review of chemical reaction pathways. Prog Energy Combust Sci 2000;26:565-608.

[122] Frenklach M. Reaction mechanism of soot formation in flames. Phys Chem Chem Phys 2002;4:2028-37.

[123] McEnally CS, Pfefferle LD, Atakan B, Kohse-Höinghaus K. Studies of aromatic hydrocarbon formation mechanisms in flames: Progress towards closing the fuel gap. Prog Energy Combust Sci 2006;32:247-94.

[124] Tree DR, Svensson KI. Soot processes in compression ignition engines. Prog Energy Combust Sci 2007;33:272-309.

[125] D'Anna A. Combustion-formed nanoparticles. Proc Combust Inst 2009;32:593-613.

[126] Michelsen HA, Schulz C, Smallwood GJ, Will S. Laser-induced incandescence: Particulate diagnostics for combustion, atmospheric, and industrial applications. Prog Energy Combust Sci 2015;51:2-48.

[127] Desgroux P, Mercier X, Thomson KA. Study of the formation of soot and its precursors in flames using optical diagnostics. Proc Combust Inst 2013;34:1713-38.

[128] Michelsen HA. Probing soot formation, chemical and physical evolution, and oxidation: A review of in situ diagnostic techniques and needs. Proc Combust Inst 2017;36:717-35.

[129] Homann KH, Wagner HG. Some new aspects of the mechanism of carbon formation in premixed flames. Proc Combust Inst 1967;11:371-9.

[130] Bonne U, Homann KH, Wagner HG. Carbon formation in premixed flames. Proc Combust Inst 1965;10:503-12.

[131] Calcote HF. Mechanisms of soot nucleation in flames-A critical review. Combust Flame 1981;42:215-42.

[132] Delfau L, Michaud P, Barassin A. Formation of Small and Large Positive Ions in Rich and

Sooting Low-Pressure Ethylene and Acetylene Premixed Flames. Combust Sci Technol

1979;20:165-77.

[133] Homann KH. Charged particles in sooting flames I. Determination of mass distributions and number densities in $\mathrm{C}_{2} \mathrm{H}_{2}-\mathrm{O}_{2}$ flames. Berichte der Bunsengesellschaft für physikalische Chemie 1979;83:738-45.

[134] Kern RD, Singh HJ, Xie K. Identification of chemi-ions formed by reactions of deuterated fuels in the reflected shock zone. J Phys Chem 1990;94:3333-5.

[135] Stein SE, Fahr A. High-temperature stabilities of hydrocarbons. J Phys Chem 1985;89:371425.

[136] Johansson KO, Lai JYW, Skeen SA, Popolan-Vaida DM, Wilson KR, Hansen N, Violi A, Michelsen HA. Soot precursor formation and limitations of the stabilomer grid. Proc Combust Inst 2015;35:1819-26.

[137] Johansson KO, Dillstrom T, Elvati P, Campbell MF, Schrader PE, Popolan-Vaida DM, Richards-Henderson NK, Wilson KR, Violi A, Michelsen HA. Radical-radical reactions, pyrene nucleation, and incipient soot formation in combustion. Proc Combust Inst 2017;36:799-806. [138] Johansson KO, Head-Gordon MP, Schrader PE, Wilson KR, Michelsen HA. Resonancestabilized hydrocarbon-radical chain reactions may explain soot inception and growth. Science 2018;361:997-1000.

[139] Graham SC, Homer JB, Rosenfeld JLJ. The Formation and Coagulation of Soot Aerosols Generated by the Pyrolysis of Aromatic Hydrocarbons. P Roy Soc A - Math Phy 1975;344:259-85. [140] Wang Y, Raj A, Chung SH. Soot modeling of counterflow diffusion flames of ethylene-based binary mixture fuels. Combust Flame 2015;162:586-96.

[141] Frenklach M, Clary DW, Gardiner Jr WC, Stein SE. Detailed kinetic modeling of soot formation in shock-tube pyrolysis of acetylene. Proc Combust Inst 1985;20:887-901. 
[142] Frenklach M, Clary DW, Yuan T, Gardiner WC, Stein SE. Mechanism of Soot Formation in Acetylene-Oxygen Mixtures. Combust Sci Technol 1986;50:79-115.

[143] Colket MB. The pyrolysis of acetylene and vinylacetylene in a single-pulse shock tube. Proc Combust Inst 1988;21:851-64.

[144] Callear AB, Smith GB. Addition of atomic hydrogen to acetylene. Chain reactions of the vinyl radical. Chem Phys Lett 1984;105:119-22.

[145] Weissman M, Benson SW. Pyrolysis of methyl chloride, a pathway in the chlorine-catalyzed polymerization of methane. Int J Chem Kinet 1984;16:307-33.

[146] Frenklach M, Warnatz J. Detailed Modeling of PAH Profiles in a Sooting Low-Pressure Acetylene Flame. Combust Sci Technol 1987;51:265-83.

[147] Harris SJ, Weiner AM, Blint RJ. Formation of small aromatic molecules in a sooting ethylene flame. Combust Flame 1988;72:91-109.

[148] Cole JA, Bittner JD, Longwell JP, Howard JB. Formation mechanisms of aromatic compounds in aliphatic flames. Combust Flame 1984;56:51-70.

[149] Westmoreland PR, Dean AM, Howard JB, Longwell JP. Forming Benzene in Flames by Chemically Activated Isomerization. J Phys Chem 1989;93:8171-80.

[150] Westmoreland PR, Howard JB, Longwell JP. Tests of published mechanisms by comparison with measured laminar flame structure in fuel-rich acetylene combustion. Proc Combust Inst 1988;21:773-82.

[151] Westmoreland PR, Howard JB, Longwell JP, Dean AM. Prediction of rate constants for combustion and pyrolysis reactions by bimolecular QRRK. AICHE J 1986;32:1971-9.

[152] Miller JA, Melius CF. Kinetic and Thermodynamic Issues in the Formation of AromaticCompounds in Flames of Aliphatic Fuels. Combust Flame 1992;91:21-39.

[153] Stein SE, Walker JA, Suryan MM, Fahr A. A new path to benzene in flames. Proc Combust Inst 1991;23:85-90.

[154] Marinov NM, Pitz WJ, Westbrook CK, Castaldi MJ, Senkan SM. Modeling of aromatic and polycyclic aromatic hydrocarbon formation in premixed methane and ethane flames. Combust Sci Technol 1996;116:211-87.

[155] Castaldi MJ, Marinov NM, Melius CF, Huang J, Senkan SM, Pit WJ, Westbrook CK. Experimental and modeling investigation of aromatic and polycyclic aromatic hydrocarbon formation in a premixed ethylene flame. Proc Combust Inst 1996;26:693-702.

[156] Richter H, Howard JB. Formation and consumption of single-ring aromatic hydrocarbons and their precursors in premixed acetylene, ethylene and benzene flames. Phys Chem Chem Phys 2002;4:2038-55.

[157] Atakan B, Hartlieb AT, Brand J, Kohse-Höinghaus K. An experimental investigation of premixed fuel-rich lowpressure propene/oxygen/argon flames by laser spectroscopy and molecularbeam mass spectrometry. Proc Combust Inst 1998;27:435-44.

[158] Kamphus M, Braun-Unkhoff M, Kohse-Höinghaus K. Formation of small PAHs in laminar premixed low-pressure propene and cyclopentene flames: Experiment and modeling. Combust Flame 2008;152:28-59.

[159] Lamprecht A, Atakan B, Kohse-Höinghaus K. Fuel-rich propene and acetylene flames: A comparison of their flame chemistries. Combust Flame 2000;122:483-91.

[160] Marinov NM, Castaldi MJ, Melius CF, Tsang W. Aromatic and polycyclic aromatic hydrocarbon formation in a premixed propane flame. Combust Sci Technol 1997;128:295-342.

[161] Marinov NM, Pitz WJ, Westbrook CK, Vincitore AM, Castaldi MJ, Senkan SM, Melius CF. Aromatic and polycyclic aromatic hydrocarbon formation in a laminar premixed n-butane flame. Combust Flame 1998;114:192-213.

[162] Alatorre GG, Bohm H, Atakan B, Kohse-Höinghaus K. Experimental and modelling study of 1-pentene combustion at fuel-rich conditions. Z Phys Chem 2001;215:981-95. 
[163] Atakan B, Lamprecht A, Kohse-Höinghaus K. An experimental study of fuel-rich 1,3pentadiene and acetylene/propene flames. Combust Flame 2003;133:431-40.

[164] Dagaut P, Cathonnet M. A comparative study of the kinetics of benzene formation from unsaturated $\mathrm{C}_{2}$ to $\mathrm{C}_{4}$ hydrocarbons. Combust Flame 1998;113:620-3.

[165] Hwang JY, Chung SH, Lee W. Effects of oxygen and propane addition on soot formation in counterflow ethylene flames and the role of C-3 chemistry. Symp (Int) Combust 1998:1531-8.

[166] Miller JA, Klippenstein SJ. The recombination of propargyl radicals and other reactions on a $\mathrm{C}_{6} \mathrm{H}_{6}$ potential. J Phys Chem A 2003;107:7783-99.

[167] Hansen N, Miller JA, Kasper T, Kohse-Höinghaus K, Westmoreland PR, Wang J, Cool TA. Benzene formation in premixed fuel-rich 1,3-butadiene flames. Proc Combust Inst 2009;32:623-30. [168] Georgievskii Y, Miller JA, Klippenstein SJ. Association rate constants for reactions between resonance-stabilized radicals: $\mathrm{C}_{3} \mathrm{H}_{3}+\mathrm{C}_{3} \mathrm{H}_{3}, \mathrm{C}_{3} \mathrm{H}_{3}+\mathrm{C}_{3} \mathrm{H}_{5}$, and $\mathrm{C}_{3} \mathrm{H}_{5}+\mathrm{C}_{3} \mathrm{H}_{5}$. Phys Chem Chem Phys 2007;9:4259-68.

[169] Pope CJ, Miller JA. Exploring old and new benzene formation pathways in low-pressure premixed flames of aliphatic fuels. Proc Combust Inst 2000;28:1519-27.

[170] Melius CF, Colvin ME, Marinov NM, Pit WJ, Senkan SM. Reaction mechanisms in aromatic hydrocarbon formation involving the $\mathrm{C} 5 \mathrm{H} 5$ cyclopentadienyl moiety. Proc Combust Inst 1996;26:685-92.

[171] Hansen N, Miller JA, Westmoreland PR, Kasper T, Kohse-Höinghaus K, Wang J, Cool TA. Isomer-specific combustion chemistry in allene and propyne flames. Combust Flame 2009; 156:2153-64.

[172] Hansen N, Cool TA, Westmoreland PR, Kohse-Höinghaus K. Recent contributions of flamesampling molecular-beam mass spectrometry to a fundamental understanding of combustion chemistry. Prog Energy Combust Sci 2009;35:168-91.

[173] Hansen N, Miller JA, Klippenstein SJ, Westmoreland PR, Kohse-Höinghaus K. Exploring formation pathways of aromatic compounds in laboratory-based model flames of aliphatic fuels. Combust Explosion Shock Waves 2012;48:508-15.

[174] Moshammer K, Seidel L, Wang Y, Selim H, Sarathy SM, Mauss F, Hansen N. Aromatic ring formation in opposed-flow diffusive 1,3-butadiene flames. Proc Combust Inst 2017;36:947-55.

[175] Skeen SA, Yang B, Jasper AW, Pitz WJ, Hansen N. Chemical Structures of Low-Pressure Premixed Methylcyclohexane Flames as Benchmarks for the Development of a Predictive Combustion Chemistry Model. Energ Fuel 2011;25:5611-25.

[176] Wang Z, Ye L, Yuan W, Zhang L, Wang Y, Cheng Z, Zhang F, Qi F. Experimental and kinetic modeling study on methylcyclohexane pyrolysis and combustion. Combust Flame 2014;161:84-100.

[177] Li W, Law ME, Westmoreland PR, Kasper T, Hansen N, Kohse-Höinghaus K. Multiple benzene-formation paths in a fuel-rich cyclohexane flame. Combust Flame 2011;158:2077-89. [178] Hansen N, Kasper T, Yang B, Cool TA, Li W, Westmoreland PR, Osswald P, Kohse-

Höinghaus K. Fuel-structure dependence of benzene formation processes in premixed flames fueled by $\mathrm{C}(6) \mathrm{H}(12)$ isomers. Proc Combust Inst 2011;33:585-92.

[179] Wang Z, Cheng Z, Yuan W, Cai J, Zhang L, Zhang F, Qi F, Wang J. An experimental and kinetic modeling study of cyclohexane pyrolysis at low pressure. Combust Flame 2012;159:224353.

[180] Silke EJ, Pitz WJ, Westbrook CK, Ribaucour M. Detailed chemical kinetic modeling of cyclohexane oxidation. J Phys Chem A 2007;111:3761-75.

[181] Nawdiyal A, Hansen N, Zeuch T, Seidel L, Mauß F. Experimental and modelling study of speciation and benzene formation pathways in premixed 1-hexene flames. Proc Combust Inst 2015;35:325-32.

[182] Moskaleva LV, Mebel AM, Lin MC. The $\mathrm{CH}_{3}+\mathrm{C}_{5} \mathrm{H}_{5}$ reaction: A potential source of benene at high temperatures. Proc Combust Inst 1996;26:521-6. 
[183] Lamprecht A, Atakan B, Kohse-Höinghaus K. Fuel-rich flame chemistry in low-pressure cyclopentene flames. Proc Combust Inst 2000;28:1817-24.

[184] Miller JA, Pilling MJ, Troe E. Unravelling combustion mechanisms through a quantitative understanding of elementary reactions. Proc Combust Inst 2005;30:43-88.

[185] Wang ZD, Zhao L, Wang Y, Bian HT, Zhang LD, Zhang F, Li YY, Sarathy SM, Qi F. Kinetics of ethylcyclohexane pyrolysis and oxidation: An experimental and detailed kinetic modeling study. Combust Flame 2015;162:2873-92.

[186] Hansen N, Klippenstein SJ, Miller JA, Wang J, Cool TA, Law ME, Westmoreland PR, Kasper T, Kohse-Höinghaus K. Identification of C5Hx isomers in fuel-rich flames by photoionization mass spectrometry and electronic structure calculations. J Phys Chem A 2006;110:4376-88.

[187] Kazakov A, Wang H, Frenklach M. Detailed modeling of soot formation in laminar premixed ethylene flames at a pressure of 10 bar. Combust Flame 1995;100:111-20.

[188] Bockhorn H, Fetting F, Wenz HW. Investigation of the formation of high molecular hydrocarbons and soot in premixed hydrocarbon-oxygen flames. Berichte der Bunsengesellschaft für physikalische Chemie 1983;87:1067-73.

[189] Fascella S, Cavallotti C, Rota R, Carra S. The peculiar kinetics of the reaction between acetylene and the cyclopentadienyl radical. J Phys Chem A 2005;109:7546-57.

[190] Hansen N, Kasper T, Klippenstein SJ, Westmoreland PR, Law ME, Taatjes CA, KohseHöinghaus K, Wang J, Cool TA. Initial steps of aromatic ring formation in a laminar premixed fuelrich cyclopentene flame. J Phys Chem A 2007;111:4081-92.

[191] Dean AM. Detailed kinetic modeling of autocatalysis in methane pyrolysis. J Phys Chem 1990;94:1432-9.

[192] Marinov NM, Pitz WJ, Westbrook CK, Lutz AE, Vincitore AM, Senkan SM. Chemical kinetic modeling of a methane opposed-flow diffusion flame and comparison to experiments. Proc Combust Inst 1998;27:605-13.

[193] Goldaniga A, Faravelli T, Ranzi E. The kinetic modeling of soot precursors in a butadiene flame. Combust Flame 2000;122:350-8.

[194] Jin HF, Frassoldati A, Wang YZ, Zhang XY, Zeng MR, Li YY, Qi F, Cuoci A, Faravelli T. Kinetic modeling study of benzene and PAH formation in laminar methane flames. Combust Flame 2015;162:1692-711.

[195] Robinson RK, Lindstedt RP. On the chemical kinetics of cyclopentadiene oxidation. Combust Flame 2011;158:666-86.

[196] Mebel AM, Kislov VV. Can the $\mathrm{C}_{5} \mathrm{H}_{5}+\mathrm{C}_{5} \mathrm{H}_{5}->\mathrm{C}_{10} \mathrm{H}_{10}->\mathrm{C}_{10} \mathrm{H}_{9}+\mathrm{H} / \mathrm{C}_{10} \mathrm{H}_{8}+\mathrm{H}_{2}$ Reaction

Produce Naphthalene? An Ab Initio/RRKM Study. J Phys Chem A 2009;113:9825-33.

[197] Cavallotti C, Polino D. On the kinetics of the $\mathrm{C}_{5} \mathrm{H}_{5}+\mathrm{C}_{5} \mathrm{H}_{5}$ reaction. Proc Combust Inst 2013;34:557-64.

[198] Frenklach M, Wang H. Detailed modeling of soot particle nucleation and growth. Proc Combust Inst 1991;23:1559-66.

[199] Wang H, Frenklach M. Enthalpies of formation of benzenoid aromatic-Molecules and radicals. J Phys Chem 1993;97:3867-74.

[200] Frenklach M, Liu Z, Singh RI, Galimova GR, Azyazov VN, Mebel AM. Detailed, stericallyresolved modeling of soot oxidation: Role of $\mathrm{O}$ atoms, interplay with particle nanostructure, and emergence of inner particle burning. Combust Flame 2018;188:284-306.

[201] Panariello M, Apicella B, Armenante M, Bruno A, Ciajolo A, Spinelli N. Analysis of polycyclic aromatic hydrocarbon sequences in a premixed laminar flame by on-line time-of-flight mass spectrometry. Rapid Communications in Mass Spectrometry 2008;22:573-81.

[202] Liu P, Lin H, Yang Y, Shao C, Guan B, Huang Z. Investigating the role of $\mathrm{CH}_{2}$ radicals in the HACA mechanism. J Phys Chem A 2015;119:3261-8. 
[203] Raj A, Prada IDC, Amer AA, Chung SH. A reaction mechanism for gasoline surrogate fuels for large polycyclic aromatic hydrocarbons. Combust Flame 2012;159:500-15.

[204] Shukla M, Susa A, Miyoshi A, Koshi M. Role of phenyl radicals in the growth of polycyclic aromatic hydrocarbons. J Phys Chem A 2008;112:2362-9.

[205] Shukla B, Koshi M. Comparative study on the growth mechanisms of PAHs. Combust Flame 2011;158:369-75.

[206] Shukla B, Miyoshi A, Koshi M. Role of methyl radicals in the growth of PAHs. Journal of the American Society for Mass Spectrometry 2010;21:534-44.

[207] Hansen N, Schenk M, Moshammer K, Kohse-Höinghaus K. Investigating repetitive reaction pathways for the formation of polycyclic aromatic hydrocarbons in combustion processes. Combust Flame 2017;180:250-61.

[208] Schenk M, Hansen N, Vieker H, Beyer A, Goelzhaeuser A, Kohse-Hoeinghaus K. PAH formation and soot morphology in flames of C-4 fuels. Proc Combust Inst 2015;35:1761-9. [209] Colket MB, Seery DJ. Reaction mechanisms for toluene pyrolysis. Proc Combust Inst 1994;25:883-91.

[210] Böhm H, Jander H, Tanke D. PAH growth and soot formation in the pyrolysis of acetylene and benzene at high temperatures and pressures: Modeling and experiment. Proc Combust Inst 1998;27:1605-12.

[211] D'Anna A, Violi A. A kinetic model for the formation of aromatic hydrocarbons in premixed laminar flames. Proc Combust Inst 1998;27:425-33.

[212] Raj A, Al Rashidi MJ, Chung SH, Sarathy SM. PAH growth initiated by propargyl addition: Mechanism development and computational kinetics. J Phys Chem A 2014;118:2865-85.

[213] Celnik M, Raj A, West R, Patterson R, Kraft M. Aromatic site description of soot particles.

Combust Flame 2008;155:161-80.

[214] D'Anna A, Kent JH. A model of particulate and species formation applied to laminar, nonpremixed flames for three aliphatic-hydrocarbon fuels. Combust Flame 2008;152:573-87.

[215] D'Anna A. Detailed kinetic modeling of particulate formation in rich premixed flames of ethylene. Energ Fuel 2008;22:1610-9.

[216] Raj A, Celnik M, Shirley R, Sander M, Patterson R, West R, Kraft M. A statistical approach to develop a detailed soot growth model using PAH characteristics. Combust Flame 2009;156:896913.

[217] Lai JYW, Elvati P, Violi A. Stochastic atomistic simulation of polycyclic aromatic hydrocarbon growth in combustion. Phys Chem Chem Phys 2014;16:7969-79.

[218] Frenklach M. On surface growth mechanism of soot particles. Proc Combust Inst 1996;26:2285-93.

[219] Sirignano M, D’Anna A. Coagulation of combustion generated nanoparticles in low and intermediate temperature regimes: An experimental study. Proc Combust Inst 2013;34:1877-84. [220] Kroto HW, Heath JR, O'Brien SC, Curl RF, Smalley RE. C60: Buckminsterfullerene. Nature 1985;318:162-3.

[221] Zhang QL, O'Brien SC, Heath JR, Liu Y, Curl RF, Kroto HW, Smalley RE. Reactivity of large carbon clusters: Spheroidal carbon shells and their possible relevance to the formation and morphology of soot. J. Phys. Chem. 1986:525-8.

[222] Homann KH. Fullerenes and soot formation - New pathways to large particles in flames. Angew Chem Int Edit 1998;37:2435-51.

[223] Ahrens J, Kovacs R, Shafranovskii EA, Homann KH. Online multiphoton ionization massspectrometry applied to PAH and fullerenes in flames. Ber Bunsen Phys Chem 1994;98:265-8. [224] Bachmann M, Griesheimer J, Homann KH. The formation of C60 and its precursors in naphthalene flames. Chem Phys Lett 1994;223:506-10.

[225] Bachmann M, Wiese W, Homann KH. PAH and aromers: Precursors of fullerenes and soot. Proc Combust Inst 1996:2259-67. 
[226] Bachmann M, Wiese W, Homann KH. Fullerenes versus soot in benzene flames. Combust Flame 1995;101:548-50.

[227] Kroto H. Space, stars, C60, and soot. Science 1988;242:1139-45.

[228] Frenklach M, Ebert LB. Comment on the proposed role of spheroidal carbon clusters in soot formation. J Phys Chem 1988;92:561-3.

[229] Stevenson R. The morphology and crystallography of diesel particulate emissions. Carbon 1982;20:359-65.

[230] Abid AD, Tolmachoff ED, Phares DJ, Wang H, Liu Y, Laskin A. Size distribution and morphology of nascent soot in premixed ethylene flames with and without benzene doping. Proc Combust Inst 2009;32:681-8.

[231] Camacho J, Lieb S, Wang H. Evolution of size distribution of nascent soot in n- and i-butanol flames. Proc Combust Inst 2013;34:1853-60.

[232] Tang Q, Mei J, You X. Effects of CO2 addition on the evolution of particle size distribution functions in premixed ethylene flame. Combust Flame 2016;165:424-32.

[233] Gu C, Lin H, Camacho J, Lin B, Shao C, Li R, Gu H, Guan B, Huang Z, Wang H. Particle size distribution of nascent soot in lightly and heavily sooting premixed ethylene flames. Combust Flame 2016;165:177-87.

[234] Zhao B, Yang ZW, Johnston MV, Wang H, Wexler AS, Balthasar M, Kraft M. Measurement and numerical simulation of soot particle size distribution functions in a laminar premixed ethyleneoxygen-argon flame. Combust Flame 2003;133:173-88.

[235] Lowe JS, Lai JYW, Elvati P, Violi A. Towards a predictive model for polycyclic aromatic hydrocarbon dimerization propensity. Proc Combust Inst 2015;35:1827-32.

[236] Sabbah H, Biennier L, Klippenstein SJ, Sims IR, Rowe BR. Exploring the role of PAHs in the formation of soot: Pyrene dimerization. J Phys Chem Lett 2010;1:2962-7.

[237] Iavarone S, Pascazio L, Sirignano M, De Candia A, Fierro A, de Arcangelis L, D'Anna A. Molecular dynamics simulations of incipient carbonaceous nanoparticle formation at flame conditions. Combust Theor Model 2017;21:49-61.

[238] Herdman JD, Miller JH. Intermolecular potential calculations for polynuclear aromatic hydrocarbon clusters. J Phys Chem A 2008;112:6249-56.

[239] Chen DP, Totton TS, Akroyd JWJ, Mosbach S, Kraft M. Size-dependent melting of polycyclic aromatic hydrocarbon nano-clusters: A molecular dynamics study. Carbon 2014;67:7991.

[240] Chen D, Akroyd J, Mosbach S, Opalka D, Kraft M. Solid-liquid transitions in homogenous ovalene, hexabenzocoronene and circumcoronene clusters: A molecular dynamics study. Combust Flame 2015;162:486-95.

[241] Schuetz CA, Frenklach M. Nucleation of soot: Molecular dynamics simulations of pyrene dimerization. Proc Combust Inst 2002;29:2307-14.

[242] Vincitore AM, Senkan SM. Polycyclic aromatic hydrocarbon formation in opposed flow diffusion flames of ethane. Combust Flame 1998;114:259-66.

[243] Chung S-H, Violi A. Peri-condensed aromatics with aliphatic chains as key intermediates for the nucleation of aromatic hydrocarbons. Proc Combust Inst 2011;33:693-700.

[244] Elvati P, Violi A. Thermodynamics of poly-aromatic hydrocarbon clustering and the effects of substituted aliphatic chains. Proc Combust Inst 2013;34:1837-43.

[245] Mao Q, van Duin ACT, Luo KH. Formation of incipient soot particles from polycyclic aromatic hydrocarbons: A ReaxFF molecular dynamics study. Carbon 2017;121:380-8.

[246] Dubey AK, Tezuka T, Hasegawa S, Nakamura H, Maruta K. Study on sooting behavior of premixed $\mathrm{C} 1-\mathrm{C} 4 \mathrm{n}$-alkanes/air flames using a micro flow reactor with a controlled temperature profile. Combust Flame 2016;174:100-10. 
[247] Nakamura H, Suzuki S, Tezuka T, Hasegawa S, Maruta K. Sooting limits and PAH formation of n-hexadecane and 2,2,4,4,6,8,8-heptamethylnonane in a micro flow reactor with a controlled temperature profile. Proc Combust Inst 2015;35:3397-404.

[248] Zhang HB, You XQ, Wang HM, Law CK. Dimerization of Polycyclic Aromatic Hydrocarbons in Soot Nucleation. J Phys Chem A 2014;118:1287-92.

[249] Koley D, Arunan E, Ramakrishnan S. Computational investigations on covalent dimerization/oligomerization of polyacenes: Is it relevant to soot formation? J Comput Chem 2012;33:1762-72.

[250] Allamandola LJ, Tielens AGGM, Barker JR. Polycyclic aromatic hydrocarbons and the unidentified infrared emission bands: Auto exhaust along the Milky Way. Astrophys J

1985;290:L25-L8.

[251] Snow TP, Le Page V, Keheyan Y, Bierbaum VM. The interstellar chemistry of PAH cations. Nature 1998;391:259.

[252] Sloan GC, Hayward TL, Allamandola LJ, Bregman JD, DeVito B, Hudgins DM. Direct spectroscopic evidence for ionized polycyclic aromatic hydrocarbons in the interstellar medium. Astrophys J 1999;513:L65-8.

[253] Serra G, Chaudret B, Saillard Y, Le Beuze A, Rabaa H, Ristorcelli I, Klotz A. Organometallic chemistry in the interstellar medium. I - Are organometallic reactions efficient processes in astrochemistry? Astron Astrophys 1992;260:489-93.

[254] Marty P, Serra G, Chaudret B, Ristorcelli I. Iron-aromatics coordination in molecular clouds. Astron Astrophys 1994;282:916-23.

[255] Calcote HF. Ion and electron profiles in flames. Proc Combust Inst 1963;9:622-37.

[256] Calcote HF. Ion production and recombination in flames. Proc Combust Inst 1961;8:184-99.

[257] Chen D, Wang H. Cation $-\pi$ Interactions between Flame Chemi-ions and Aromatic

Compounds. Energ Fuel 2017;31:2345-52.

[258] Kolakkandy S, Pratihar S, Aquino AJA, Wang H, Hase WL. Properties of complexes formed by $\mathrm{Na}^{+}, \mathrm{Mg}^{2+}$, and $\mathrm{Fe}^{2+}$ binding with benzene molecules. J Phys Chem A 2014;118:9500-11.

[259] Demircan ÇA, Bozkaya U. Transition Metal Cation- $\pi$ Interactions: Complexes Formed by

$\mathrm{Fe} 2+, \mathrm{Co} 2+, \mathrm{Ni} 2+, \mathrm{Cu} 2+$, and Zn2+ Binding with Benzene Molecules. J Phys Chem A

2017;121:6500-9.

[260] Haynes BS, Jander H, Wagner HG. The effect of metal additives on the formation of soot in premixed flames. Proc Combust Inst 1979;17:1365-74.

[261] Raj A, Pan K, Qi H, Zhu H, Wen JZ, Croiset E. Effects of an iron pentacarbonyl additive on counterflow natural gas and ethanol flames. Energ Fuel 2015;29:5361-71.

[262] Kasper M, Siegmann K. The influence of ferrocene on PAH synthesis in acetylene and methane diffusion flames. Combust Sci Technol 1998;140:333-50.

[263] Bonczyk PA. Effect of Ferrocene on soot in a prevaporized iso-octane/air diffusion flame. Combust Flame 1991;87:233-44.

[264] Wieschnowsky U, Bockhorn H, Fetting F. Some new observations concerning the mass growth of soot in premixed hydrocarbon-oxygen flames. Proc Combust Inst 1989;22:343-52.

[265] Martin JW, Slavchov RI, Yapp EKY, Akroyd J, Mosbach S, Kraft M. The Polarization of Polycyclic Aromatic Hydrocarbons Curved by Pentagon Incorporation: The Role of the Flexoelectric Dipole. J Phys Chem C 2017;121: 27154-63.

[266] Martin JW, Botero M, Slavchov RI, Bowal K, Akroyd J, Mosbach S, Kraft M.

Flexoelectricity and the Formation of Carbon Nanoparticles in Flames. J Phys Chem C 2018;122:22210-5.

[267] Martin JW, Bowal K, Menon A, Slavchov RI, Akroyd J, Mosbach S, Kraft M. Polar curved polycyclic aromatic hydrocarbons in soot formation. Proc Combust Inst 2019;37:1117-23.

[268] Bowal K, Martin JW, Misquitta AJ, Kraft M. Ion-induced soot nucleation using a new potential for curved aromatics. Combust Sci Technol 2019:1-19. 
[269] Carbone F, Canagaratna MR, Lambe AT, Jayne JT, Worsnop DR, Gomez A. Exploratory analysis of a sooting premixed flame via on-line high resolution (APi-TOF) mass spectrometry. Proc Combust Inst 2019;37:919-26.

[270] Leung KM, Lindstedt RP, Jones WP. A simplified reaction mechanism for soot formation in nonpremixed flames. Combust Flame 1991;87:289-305.

[271] Lindstedt PR. Simplified Soot Nucleation and Surface Growth Steps for Non-Premixed Flames. In: Bockhorn H, editor. Soot Formation in Combustion: Mechanisms and Models. Berlin, Heidelberg: Springer Berlin Heidelberg; 1994. p. 417-41.

[272] Eaves NA, Dworkin SB, Thomson MJ. Assessing relative contributions of PAHs to soot mass by reversible heterogeneous nucleation and condensation. Proc Combust Inst 2017;36:935-45.

[273] Thomson M, Mitra T. A radical approach to soot formation. Science 2018;361:978-9. [274] Haynes BS, Wagner HG. The surface growth phenomenon in soot formation. Z Phys Chem 1982;133:201.

[275] Kennedy IM. The evolution of a soot aerosol in a counterflow diffusion flame. Combust Flame 1987;68:1-16.

[276] Harris SJ, Weiner AM. Surface growth of soot particles in premixed ethylene/air flames. Combust Sci Technol 1983;31:155-67.

[277] Sunderland PB, Faeth GM. Soot formation in hydrocarbon/air laminar jet diffusion flames. Combust Flame 1996;105:132-46.

[278] Sunderland PB, Koylu UO, Faeth GM. Soot formation in weakly buoyant acetylene-fueled laminar jet diffusion flames burning in air. Combust Flame 1995;100:310-22.

[279] Lin KC, Sunderland PB, Faeth GM. Soot nucleation and growth in acetylene air laminar coflowing jet diffusion flames. Combust Flame 1996;104:369-75.

[280] Appel J, Bockhorn H, Frenklach M. Kinetic modeling of soot formation with detailed chemistry and physics: Laminar premixed flames of C-2 hydrocarbons. Combust Flame 2000;121:122-36.

[281] Hwang JY, Chung SH. Growth of soot particles in counterflow diffusion flames of ethylene. Combust Flame 2001;125:752-62.

[282] Dworkin SB, Zhang QA, Thomson MJ, Slavinskaya NA, Riedel U. Application of an enhanced PAH growth model to soot formation in a laminar coflow ethylene/air diffusion flame. Combust Flame 2011;158:1682-95.

[283] Khosousi A, Dworkin SB. Soot surface reactivity during surface growth and oxidation in laminar diffusion flames. Combust Flame 2015;162:4523-32.

[284] Liu F, He X, Ma X, Zhang Q, Thomson MJ, Guo H, Smallwood GJ, Shuai S, Wang J. An experimental and numerical study of the effects of dimethyl ether addition to fuel on polycyclic aromatic hydrocarbon and soot formation in laminar coflow ethylene/air diffusion flames. Combust Flame 2011;158:547-63.

[285] Chen D, Akroyd J, Mosbach S, Kraft M. Surface reactivity of polycyclic aromatic hydrocarbon clusters. Proc Combust Inst 2015;35:1811-8.

[286] Chen D, Totton TS, Akroyd J, Mosbach S, Kraft M. Phase change of polycyclic aromatic hydrocarbon clusters by mass addition. Carbon 2014;77:25-35.

[287] Saffaripour M, Veshkini A, Kholghy M, Thomson MJ. Experimental investigation and detailed modeling of soot aggregate formation and size distribution in laminar coflow diffusion flames of Jet A-1, a synthetic kerosene, and n-decane. Combust Flame 2014;161:848-63.

[288] Veshkini A, Eaves NA, Dworkin SB, Thomson MJ. Application of PAH-condensation reversibility in modeling soot growth in laminar premixed and nonpremixed flames. Combust Flame 2016; 167:335-52.

[289] Eaves NA, Dworkin SB, Thomson MJ. The importance of reversibility in modeling soot nucleation and condensation processes. Proc Combust Inst 2015;35:1787-94. 
[290] D'Anna A, Commodo M, Sirignano M, Minutolo P, Pagliara R. Particle formation in opposed-flow diffusion flames of ethylene: An experimental and numerical study. Proc Combust Inst 2009;32:793-801.

[291] Frenklach M, Schuetz CA, Ping J. Migration mechanism of aromatic-edge growth. Proc Combust Inst 2005;30:1389-96.

[292] Singh R, Frenklach M. A mechanistic study of the influence of graphene curvature on the rate of high-temperature oxidation by molecular oxygen. Carbon 2016;101:203-12.

[293] Celnik MS, Sander M, Raj A, West RH, Kraft M. Modelling soot formation in a premixed flame using an aromatic-site soot model and an improved oxidation-rate. Proc Combust Inst 2009;32:639-46.

[294] Frenklach M, Moriarty NW, Brown NJ. Hydrogen migration in polyaromatic growth. Proc Combust Inst 1998;27:1655-61.

[295] Violi A. Cyclodehydrogenation reactions to cyclopentafused polycyclic aromatic hydrocarbons. J Phys Chem A 2005;109:7781-7.

[296] Whitesides R, Domin D, Salomón-Ferrer R, Lester WA, Frenklach M. Graphene layer growth chemistry: Five- and six-member ring flip reaction. J Phys Chem A 2008;112:2125-30.

[297] Böhm H, Jander H. PAH formation in acetylene-benzene pyrolysis. Phys Chem Chem Phys 1999;1:3775-81.

[298] Raj A, Man PLW, Totton TS, Sander M, Shirley RA, Kraft M. New polycyclic aromatic hydrocarbon $(\mathrm{PAH})$ surface processes to improve the model prediction of the composition of combustion-generated PAHs and soot. Carbon 2010;48:319-32.

[299] Whitesides R, Frenklach M. Detailed kinetic Monte Carlo simulations of graphene-edge growth. J Phys Chem A 2010;114:689-703.

[300] Sander M, Patterson RIA, Braumann A, Raj A, Kraft M. Developing the PAH-PP soot particle model using process informatics and uncertainty propagation. Proc Combust Inst 2011;33:675-83.

[301] You X, Whitesides R, Zubarev D, Lester WA, Frenklach M. Bay-capping reactions: Kinetics and influence on graphene-edge growth. Proc Combust Inst 2011;33:685-92.

[302] Whitesides R, Frenklach M. Effect of reaction kinetics on graphene-edge morphology and composition. Z Phys Chem2015. p. 597.

[303] Violi A, Kubota A, Truong TN, Pitz WJ, Westbrook CK, Sarofim AF. A fully integrated kinetic monte carlo/molecular dynamics approach for the simulation of soot precursor growth. Proc Combust Inst 2002;29:2343-9.

[304] Violi A. Modeling of soot particle inception in aromatic and aliphatic premixed flames.

Combust Flame 2004;139:279-87.

[305] Violi A, Izvekov S. Soot primary particle formation from multiscale coarse-grained molecular dynamics simulation. Proc Combust Inst 2007;31:529-37.

[306] Apicella B, Pré P, Alfè M, Ciajolo A, Gargiulo V, Russo C, Tregrossi A, Deldique D,

Rouzaud JN. Soot nanostructure evolution in premixed flames by High Resolution Electron

Transmission Microscopy (HRTEM). Proc Combust Inst 2015;35:1895-902.

[307] Botero ML, Chen D, González-Calera S, Jefferson D, Kraft M. HRTEM evaluation of soot particles produced by the non-premixed combustion of liquid fuels. Carbon 2016;96:459-73.

[308] Martin JW, Bowal K, Menon A, Slavchov RI, Akroyd J, Mosbach S, Kraft M. Polar curved polycyclic aromatic hydrocarbons in soot formation. Proc Combust Inst 2018.

[309] Vander Wal RL, Tomasek AJ. Soot oxidation: Dependence upon initial nanostructure.

Combust Flame 2003;134:1-9.

[310] Yapp EKY, Wells CG, Akroyd J, Mosbach S, Xu R, Kraft M. Modelling PAH curvature in laminar premixed flames using a detailed population balance model. Combust Flame 2017;176:17280 . 
[311] Xu F, Sunderland PB, Faeth GM. Soot formation in laminar premixed ethylene/air flames at atmospheric pressure. Combust Flame 1997;108:471-93.

[312] Faeth GM, Koylu UO. Soot morphology and optical properties in nonpremixed turbulent flame environments. Combust Sci Technol 1995;108:207-29.

[313] Mitchell P, Frenklach M. Monte Carlo simulation of soot aggregation with simultaneous surface growth-Why primary particles appear spherical. Proc Combust Inst 1998;27:1507-14.

[314] Mitchell P, Frenklach M. Particle aggregation with simultaneous surface growth. Phys Rev E 2003;67:061407.

[315] Balthasar M, Frenklach M. Monte Carlo simulation of soot particle coagulation and aggregation: The effect of a realistic size distribution. Proc Combust Inst 2005;30:1467-75.

[316] Ono K, Dewa K, Matsukawa Y, Saito Y, Matsushita Y, Aoki H, Era K, Aoki T, Yamaguchi T. Experimental evidence for the sintering of primary soot particles. J Aerosol Sci 2017;105:1-9.

[317] Chen DP, Zainuddin Z, Yapp E, Akroyd J, Mosbach S, Kraft M. A fully coupled simulation of PAH and soot growth with a population balance model. Proc Combust Inst 2013;34:1827-35. [318] Zhao B, Yang ZW, Wang JJ, Johnston MV, Wang H. Analysis of soot nanoparticles in a laminar premixed ethylene flame by scanning mobility particle sizer. Aerosol Sci Tech 2003;37:611-20.

[319] Maricq MM. A comparison of soot size and charge distributions from ethane, ethylene, acetylene, and benzene/ethylene premixed flames. Combust Flame 2006;144:730-43.

[320] Echavarria CA, Sarofim AF, Lighty JS, D'Anna A. Modeling and measurements of size distributions in premixed ethylene and benzene flames. Proc Combust Inst 2009;32:705-11.

[321] Ono K, Matsukawa Y, Dewa K, Watanabe A, Takahashi K, Saito Y, Matsushita Y, Aoki H, Era K, Aoki T, Yamaguchi T. Formation mechanisms of soot from high-molecular-weight polycyclic aromatic hydrocarbons. Combust Flame 2015;162:2670-8.

[322] Singh J, Patterson RIA, Kraft M, Wang H. Numerical simulation and sensitivity analysis of detailed soot particle size distribution in laminar premixed ethylene flames. Combust Flame 2006;145:117-27.

[323] Botero ML, Eaves N, Dreyer JAH, Sheng Y, Akroyd J, Yang W, Kraft M. Experimental and numerical study of the evolution of soot primary particles in a diffusion flame. Proc Combust Inst 2018.

[324] D’Alessio A, Barone AC, Cau R, D'Anna A, Minutolo P. Surface deposition and coagulation efficiency of combustion generated nanoparticles in the size range from 1 to $10 \mathrm{~nm}$. Proc Combust Inst 2005;30:2595-603.

[325] Sgro LA, Barone AC, Commodo M, D'Alessio A, De Filippo A, Lanzuolo G, Minutolo P. Measurement of nanoparticles of organic carbon in non-sooting flame conditions. Proc Combust Inst 2009;32:689-96.

[326] Sgro LA, De Filippo A, Lanzuolo G, D’Alessio A. Characterization of nanoparticles of organic carbon (NOC) produced in rich premixed flames by differential mobility analysis. Proc Combust Inst 2007;31:631-8.

[327] Stanmore BR, Brilhac JF, Gilot P. The oxidation of soot: A review of experiments, mechanisms and models. Carbon 2001;39:2247-68.

[328] Cavaliere A, Barbella R, Ciajolo A, D'Anna A, Ragucci R. Fuel and soot oxidation in diesellike conditions. Proc Combust Inst 1994;25:167-74.

[329] Stanmore BR, Tschamber V, Brilhac JF. Oxidation of carbon by NOx, with particular reference to NO2 and N2O. Fuel 2008;87:131-46.

[330] Matarrese R, Castoldi L, Lietti L. Oxidation of model soot by NO2 and O2 in the presence of water vapor. Chem Eng Sci 2017;173:560-9.

[331] Millikan RC. Non-equilibrium soot formation in premixed flames. J Phys Chem 1962;66:7949. 
[332] Fenimore CP, Jones GW. Oxidation of soot by hydroxyl radicals. J Phys Chem 1967;71:5937.

[333] Roth P, Brandt O, Von Gersum S. High temperature oxidation of suspended soot particles verified by CO and CO2 measurements. Proc Combust Inst 1991;23:1485-91.

[334] Neoh KG, Howard JB, Sarofim AF. Effect of oxidation on the physical structure of soot. Proc Combust Inst 1985;20:951-7.

[335] Puri R, Santoro RJ, Smyth KC. The oxidation of soot and carbon-monoxide in hydrocarbon diffusion flames. Combust Flame 1994;97:125-44.

[336] Xu F, El-Leathy AM, Kim CH, Faeth GM. Soot surface oxidation in hydrocarbon/air diffusion flames at atmospheric pressure. Combust Flame 2003;132:43-57.

[337] Von Gersum S, Roth P. High temperature oxidation of soot particles by $\mathrm{O}$ atoms and $\mathrm{OH}$ radicals. J Aerosol Sci 1990;21:S31-S4.

[338] D'Anna A, Kent JH. Modeling of particulate carbon and species formation in coflowing diffusion flames of ethylene. Combust Flame 2006;144:249-60.

[339] Sirignano M, Kent J, D'Anna A. Modeling formation and oxidation of soot in nonpremixed flames. Energ Fuel 2013;27:2303-15.

[340] Edwards DE, Zubarev DY, Lester WA, Frenklach M. Pathways to soot oxidation: Reaction of $\mathrm{OH}$ with phenanthrene radicals. J Phys Chem A 2014;118:8606-13.

[341] Tokmakov IV, Lin MC. Kinetics and mechanism of the $\mathrm{OH}+\mathrm{C}_{6} \mathrm{H}_{6}$ reaction: A detailed analysis with first-principles calculations. J Phys Chem A 2002;106:11309-26.

[342] Hollman DS, Simmonett AC, Schaefer HF. The benzene+OH potential energy surface: Intermediates and transition states. Phys Chem Chem Phys 2011;13:2214-21.

[343] You X, Zubarev DY, Lester WA, Frenklach M. Thermal Decomposition of Pentacene Oxyradicals. J Phys Chem A 2011;115:14184-90.

[344] Edwards DE, You X, Zubarev DY, Lester WA, Frenklach M. Thermal decomposition of graphene armchair oxyradicals. Proc Combust Inst 2013;34:1759-66.

[345] Sediako AD, Soong C, Howe JY, Kholghy MR, Thomson MJ. Real-time observation of soot aggregate oxidation in an Environmental Transmission Electron Microscope. Proc Combust Inst 2017;36:841-51.

[346] Al-Qurashi K, Boehman AL. Impact of exhaust gas recirculation (EGR) on the oxidative reactivity of diesel engine soot. Combust Flame 2008;155:675-95.

[347] Strzelec A, Wal RLV, Thompson TN, Toops TJ, Daw CS. $\mathrm{NO}_{2}$ oxidation reactivity and burning mode of diesel particulates. Top Catal 2016;59:686-94.

[348] Song J, Alam M, Boehman AL, Kim U. Examination of the oxidation behavior of biodiesel soot. Combust Flame 2006;146:589-604.

[349] Kholghy MR, Veshkini A, Thomson MJ. The core-shell internal nanostructure of soot - A criterion to model soot maturity. Carbon 2016;100:508-36.

[350] Singh RI, Mebel AM, Frenklach M. Oxidation of graphene-edge six- and five-member rings by molecular oxygen. J Phys Chem A 2015;119:7528-47.

[351] Xu K, Ye PD. Theoretical study on the oxidation mechanism and dynamics of the zigzag graphene nanoribbon edge by oxygen and ozone. J Phys Chem C 2014;118:10400-7.

[352] Camacho J, Tao Y, Wang H. Kinetics of nascent soot oxidation by molecular oxygen in a flow reactor. Proc Combust Inst 2015;35:1887-94.

[353] Khosousi A, Dworkin SB. Detailed modelling of soot oxidation by $\mathrm{O} 2$ and $\mathrm{OH}$ in laminar diffusion flames. Proc Combust Inst 2015;35:1903-10.

[354] Guo H, Anderson PM, Sunderland PB. Optimized rate expressions for soot oxidation by $\mathrm{OH}$ and O2. Fuel 2016;172:248-52.

[355] Sirignano M, Ghiassi H, D'Anna A, Lighty JS. Temperature and oxygen effects on oxidationinduced fragmentation of soot particles. Combust Flame 2016;171:15-26. 
[356] Ghiassi H, Toth P, Jaramillo IC, Lighty JS. Soot oxidation-induced fragmentation: Part 1: The relationship between soot nanostructure and oxidation-induced fragmentation. Combust Flame 2016;163:179-87.

[357] Ghiassi H, Jaramillo IC, Toth P, Lighty JS. Soot oxidation-induced fragmentation: Part 2: Experimental investigation of the mechanism of fragmentation. Combust Flame 2016;163:170-8. [358] Echavarria CA, Jaramillo IC, Sarofim AF, Lighty JS. Studies of soot oxidation and fragmentation in a two-stage burner under fuel-lean and fuel-rich conditions. Proc Combust Inst 2011;33:659-66.

[359] Raj A, Yang SY, Cha D, Tayouo R, Chung SH. Structural effects on the oxidation of soot particles by O-2: Experimental and theoretical study. Combust Flame 2013;160:1812-26.

[360] Zhang Y, Boehman AL. Oxidation behavior of soot generated from the combustion of methyl 2-butenoate in a co-flow diffusion flame. Combust Flame 2013;160:112-9.

[361] Tighe CJ, Twigg MV, Hayhurst AN, Dennis JS. The kinetics of oxidation of diesel soots and a carbon black (Printex $U$ ) by $\mathrm{O} 2$ with reference to changes in both size and internal structure of the spherules during burnout. Carbon 2016;107:20-35.

[362] Raj A, da Silva GR, Chung SH. Reaction mechanism for the free-edge oxidation of soot by O-2. Combust Flame 2012;159:3423-36.

[363] Zhou C-W, Kislov VV, Mebel AM. Reaction mechanism of naphthyl radicals with molecular oxygen. 1. Theoretical study of the potential energy surface. J Phys Chem A 2012;116:1571-85.

[364] Kislov VV, Singh RI, Edwards DE, Mebel AM, Frenklach M. Rate coefficients and product branching ratios for the oxidation of phenyl and naphthyl radicals: A theoretical RRKM-ME study. Proc Combust Inst 2015;35:1861-9.

[365] Sendt K, Haynes BS. Density functional study of the reaction of $\mathrm{O}_{2}$ with a single site on the zigzag edge of graphene. Proc Combust Inst 2011;33:1851-8.

[366] Sendt K, Haynes BS. Density functional study of the chemisorption of $\mathrm{O}_{2}$ on the armchair surface of graphite. Proc Combust Inst 2005;30:2141-9.

[367] Sendt K, Haynes BS. Density functional study of the chemisorption of $\mathrm{O}_{2}$ on the zig-zag surface of graphite. Combust Flame 2005;143:629-43.

[368] Chaparala SV, Raj A. Reaction mechanism for the oxidation of zigzag site on polycyclic aromatic hydrocarbons in soot by O2. Combust Flame 2016;165:21-33.

[369] Müller JO, Su DS, Jentoft RE, Kröhnert J, Jentoft FC, Schlögl R. Morphology-controlled reactivity of carbonaceous materials towards oxidation. Catal Today 2005;102-103:259-65.

[370] Vander Wal RL, Yezerets A, Currier NW, Kim DH, Wang CM. HRTEM Study of diesel soot collected from diesel particulate filters. Carbon 2007;45:70-7.

[371] Raj A, Tayouo R, Cha D, Li L, Ismail MA, Chung SH. Thermal fragmentation and deactivation of combustion-generated soot particles. Combust Flame 2014;161:2446-57.

[372] Sirignano M, Kent J, D'Anna A. Further experimental and modelling evidences of soot fragmentation in flames. Proc Combust Inst 2015;35:1779-86.

[373] Zhang Q, Thomson MJ, Guo H, Liu F, Smallwood GJ. Modeling of oxidation-driven soot aggregate fragmentation in a laminar coflow diffusion flame. Combust Sci Technol 2010;182:491504.

[374] Harris SJ, Maricq MM. The role of fragmentation in defining the signature size distribution of diesel soot. J Aerosol Sci 2002;33:935-42.

[375] Salenbauch S, Cuoci A, Frassoldati A, Saggese C, Faravelli T, Hasse C. Modeling soot formation in premixed flames using an Extended Conditional Quadrature Method of Moments. Combust Flame 2015;162:2529-43.

[376] Lindstedt RP, Waldheim BBO. Modeling of soot particle size distributions in premixed stagnation flow flames. Proc Combust Inst 2013;34:1861-8. 
[377] Kalvakala KC, Katta VR, Aggarwal SK. Effects of oxygen-enrichment and fuel unsaturation on soot and NOx emissions in ethylene, propane, and propene flames. Combust Flame 2018;187:217-29.

[378] Xu L, Yan F, Zhou M, Wang Y, Chung SH. Experimental and soot modeling studies of ethylene counterflow diffusion flames: Non-monotonic influence of the oxidizer composition on soot formation. Combust Flame 2018;197:304-18.

[379] Aubagnac-Karkar D, El Bakali A, Desgroux P. Soot particles inception and PAH condensation modelling applied in a soot model utilizing a sectional method. Combust Flame 2018; 189:190-206.

[380] Chung SH, Violi A. Insights on the nanoparticle formation process in counterflow diffusion flames. Carbon 2007;45:2400-10.

[381] Elvati P, Dillstrom VT, Violi A. Oxygen driven soot formation. Proc Combust Inst 2017;36:825-32.

[382] Balthasar M, Kraft M. A stochastic approach to calculate the particle size distribution function of soot particles in laminar premixed flames. Combust Flame 2003;133:289-98.

[383] Singh J, Balthasar M, Kraft M, Wagner W. Stochastic modeling of soot particle size and age distributions in laminar premixed flames. Proc Combust Inst 2005;30:1457-65.

[384] Patterson RIA, Singh J, Balthasar M, Kraft M, Wagner W. Extending stochastic soot simulation to higher pressures. Combust Flame 2006;145:638-42.

[385] Celnik M, Patterson R, Kraft M, Wagner W. Coupling a stochastic soot population balance to gas-phase chemistry using operator splitting. Combust Flame 2007;148:158-76.

[386] Frenklach M. Method of moments with interpolative closure. Chem Eng Sci 2002;57:222939.

[387] Guo HS, Thomson KA, Smallwood GJ. On the effect of carbon monoxide addition on soot formation in a laminar ethylene/air coflow diffusion flame. Combust Flame 2009; 156:1135-42. [388] Kazakov A, Frenklach M. Dynamic modeling of soot particle coagulation and aggregation: Implementation with the method of moments and application to high-pressure laminar premixed flames. Combust Flame 1998;114:484-501.

[389] Salenbauch S, Sirignano M, Pollack M, D’Anna A, Hasse C. Detailed modeling of soot particle formation and comparison to optical diagnostics and size distribution measurements in premixed flames using a method of moments. Fuel 2018;222:287-93.

[390] Balthasar M, Frenklach M. Detailed kinetic modeling of soot aggregate formation in laminar premixed flames. Combust Flame 2005;140:130-45.

[391] Mueller ME, Blanquart G, Pitsch H. A joint volume-surface model of soot aggregation with the method of moments. Proc Combust Inst 2009;32:785-92.

[392] Zucca A, Marchisio DL, Barresi AA, Fox RO. Implementation of the population balance equation in CFD codes for modelling soot formation in turbulent flames. Chem Eng Sci 2006;61:87-95.

[393] Marchisio DL, Barresi AA. Investigation of soot formation in turbulent flames with a pseudobivariate population balance model. Chem Eng Sci 2009;64:294-303.

[394] Cuoci A, Frassoldati A, Faravelli T, Ranzi E. Kinetic Modeling of Soot Formation in Turbulent Nonpremixed Flames. Environmental Engineering Science 2008;25:1407-22.

[395] Cuoci A, Frassoldati A, Faravelli T, Ranzi E. Formation of soot and nitrogen oxides in unsteady counterflow diffusion flames. Combust Flame 2009;156:2010-22.

[396] Zhang Q, Thomson MJ, Guo H, Liu F, Smallwood GJ. A numerical study of soot aggregate formation in a laminar coflow diffusion flame. Combust Flame 2009;156:697-705.

[397] Yan F, Xu L, Wang Y, Park S, Sarathy SM, Chung SH. On the opposing effects of methanol and ethanol addition on PAH and soot formation in ethylene counterflow diffusion flames. Combust Flame 2019;202:228-42. 
[398] Blacha T, Di Domenico M, Gerlinger P, Aigner M. Soot predictions in premixed and nonpremixed laminar flames using a sectional approach for PAHs and soot. Combust Flame 2012;159:181-93.

[399] Jerez A, Consalvi J-L, Fuentes A, Liu F, Demarco R. Soot production modeling in a laminar coflow ethylene diffusion flame at different Oxygen Indices using a PAH-based sectional model. Fuel 2018;231:404-16.

[400] Eberle C, Gerlinger P, Aigner M. A sectional PAH model with reversible PAH chemistry for CFD soot simulations. Combust Flame 2017;179:63-73.

[401] Kennedy IM, Yam C, Rapp DC, Santoro RJ. Modeling and measurements of soot and species in a laminar diffusion flame. Combust Flame 1996;107:368-82.

[402] Chernov V, Zhang QG, Thomson MJ, Dworkin SB. Numerical investigation of soot formation mechanisms in partially-premixed ethylene-air co-flow flames. Combust Flame 2012;159:2789-98.

[403] Khosousi A, Liu F, Dworkin SB, Eaves NA, Thomson MJ, He X, Dai Y, Gao Y, Liu F, Shuai $\mathrm{S}$, Wang J. Experimental and numerical study of soot formation in laminar coflow diffusion flames of gasoline/ethanol blends. Combust Flame 2015;162:3925-33.

[404] Saffaripour M, Zabeti P, Dworkin SB, Zhang Q, Guo H, Liu F, Smallwood GJ, Thomson MJ. A numerical and experimental study of a laminar sooting coflow Jet-A1 diffusion flame. Proc Combust Inst 2011;33:601-8.

[405] Senkan S, Castaldi M. Formation of polycyclic aromatic hydrocarbons (PAH) in methane combustion: Comparative new results from premixed flames. Combust Flame 1996;107:141-50.

[406] Bhargava A, Westmoreland PR. Measured flame structure and kinetics in a fuel-rich ethylene flame. Combust Flame 1998;113:333-47.

[407] Olten N, Senkan S. Formation of polycyclic aromatic hydrocarbons in an atmospheric pressure ethylene diffusion flame. Combust Flame 1999;118:500-7.

[408] Kantrowitz A, Grey J. A high intensity source for the molecular beam. Part I. Theoretical. Rev Sci Instrum 1951;22:328-32.

[409] Kistiakowsky GB, Slichter WP. A high intensity source for the molecular beam. Part II. Experimental. Rev Sci Instrum 1951;22:333-7.

[410] Biordi JC. Molecular beam mass spectrometry for studying the fundamental chemistry of flames. Prog Energy Combust Sci 1977;3:151-73.

[411] Westmoreland PR, Law ME, Cool TA, Wang J, McIlroy A, Taatjes CA, Hansen N. Analysis of flame structure by molecular-beam mass spectrometry using electron-impact and synchrotronphoton ionization. Combust Explosion Shock Waves 2006;42:672-7.

[412] Qi F. Combustion chemistry probed by synchrotron VUV photoionization mass spectrometry. Proc Combust Inst 2013;34:33-63.

[413] Skeen SA, Yang B, Michelsen HA, Miller JA, Violi A, Hansen N. Studies of laminar opposed-flow diffusion flames of acetylene at low-pressures with photoionization mass spectrometry. Proc Combust Inst 2013;34:1067-75.

[414] Taatjes CA, Hansen N, McIlroy A, Miller JA, Senosiain JP, Klippenstein SJ, Qi F, Sheng LS, Zhang YW, Cool TA, Wang J, Westmoreland PR, Law ME, Kasper T, Kohse-Höinghaus K. Enols are common intermediates in hydrocarbon oxidation. Science 2005;308:1887-9.

[415] Bermudez G, Pfefferle L. Laser ionization time-of-flight mass spectrometry combined with residual gas analysis for the investigation of moderate temperature benzene oxidation. Combust Flame 1995;100:41-51.

[416] McEnally CS, Pfefferle LD. Aromatic and Linear Hydrocarbon Concentration Measurements in a Non-Premixed Flame. Combust Sci Technol 1996;116-117:183-209.

[417] Marangos JP, Shen N, Ma H, Hutchinson MHR, Connerade JP. Broadly tunable vacuumultraviolet radiation source employing resonant enhanced sum-difference frequency mixing in krypton. J OPT SOC AM B 1990;7:1254-9. 
[418] Qi F, McIlroy A. Identifying combustion intermediates via tunable vacuum ultraviolet photoionization mass spectrometry. Combust Sci Technol 2005;177:2021-37.

[419] Werner JH, Cool TA. The combustion of trichloroethylene studied with vacuum ultraviolet photoionization mass spectrometry. Proc Combust Inst 1998;27:413-23.

[420] Werner JH, Cool TA. The kinetics of the combustion of trichloroethylene for low $\mathrm{Cl} / \mathrm{H}$ ratios. Combust Flame 2000;120:125-42.

[421] Cool TA, Williams BA. Ultrasensitive detection of chlorinated hydrocarbons by resonance ionization. Combust Sci Technol 1992;82:67-85.

[422] Williams BA, Tanada TN, Cool TA. Resonance ionization detection limits for hazardous emissions. Proc Combust Inst 1992;24:1587-96.

[423] Kasper TS, Osswald P, Kamphus M, Kohse-Höinghaus K. Ethanol flame structure investigated by molecular beam mass spectrometry. Combust Flame 2007;150:220-31.

[424] Hepp H, Siegmann K, Sattler K. New aspects of growth mechanisms for polycyclic aromatic hydrocarbons in diffusion flames. Chem Phys Lett 1995;233:16-22.

[425] Siegmann K, Hepp H, Sattler K. Reactive dimerization: A new PAH growth mechanism in flames. Combust Sci Technol 1995;109:165-81.

[426] Ahrens J, Keller A, Kovacs R, Homann KH. Large molecules, radicals, ions, and small soot particles in fuel-rich hydrocarbon flames: Part III: REMPI mass spectrometry of large flame PAHs and fullerenes and their quantitative calibration through sublimation. Berichte der

Bunsengesellschaft für physikalische Chemie 1998;102:1823-39.

[427] Gittins CM, Castaldi MJ, Senkan SM, Rohlfing EA. Real-time quantitative analysis of combustion-generated polycyclic aromatic hydrocarbons by resonance-enhanced multiphoton ionization time-of-flight mass spectrometry. Anal Chem 1997;69:286-93.

[428] Streibel T, Zimmermann R. Resonance-enhanced multiphoton ionization mass spectrometry (REMPI-MS): Applications for process analysis. Annu Rev Anal Chem 2014;7:361-81.

[429] Hartlieb AT, Atakan B, Kohse-Höinghaus K. Effects of a sampling quartz nozzle on the flame structure of a fuel-rich low-pressure propene flame. Combust Flame 2000;121:610-24.

[430] Goldenstein CS, Spearrin RM, Jeffries JB, Hanson RK. Infrared laser-absorption sensing for combustion gases. Prog Energy Combust Sci 2017;60:132-76.

[431] Hanson RK, Davidson DF. Recent advances in laser absorption and shock tube methods for studies of combustion chemistry. Prog Energy Combust Sci 2014;44:103-14.

[432] Kohse-Höinghaus K. Laser techniques for the quantitative detection of reactive intermediates in combustion systems. Prog Energy Combust Sci 1994;20:203-79.

[433] Kiefer J, Ewart P. Laser diagnostics and minor species detection in combustion using resonant four-wave mixing. Prog Energy Combust Sci 2011;37:525-64.

[434] Hanson RK, Spearrin RM, Goldenstein CS. Spectroscopy and Optical Diagnostics for Gases. Switzerland: Springer International; 2016.

[435] Wang S, Parise T, Johnson SE, Davidson DF, Hanson RK. A new diagnostic for hydrocarbon fuels using 3.41- $\mu \mathrm{m}$ diode laser absorption. Combust Flame 2017;186:129-39.

[436] Zalicki P, Zare RN. Cavity ring-down spectroscopy for quantitative absorption measurements. J Chem Phys 1995;102:2708-17.

[437] Liu X, Zhang G, Huang Y, Wang Y, Qi F. Two-dimensional temperature and carbon dioxide concentration profiles in atmospheric laminar diffusion flames measured by mid-infrared direct absorption spectroscopy at 4.2 $\mu \mathrm{m}$. Appl Phys B 2018;124:61.

[438] Beretta F, Cincotti V, Dalessio A, Menna P. Ultraviolet and Visible Fluorescence in the Fuel Pyrolysis Regions of Gaseous-Diffusion Flames. Combust Flame 1985;61:211-8.

[439] Zizak G, Cignoli F, Montas G, Benecchi S, Donde R. Detection of aromatic hydrocarbons in the exhaust gases of a gasoline i.c. engine by laser-induced fluorescence technique. Recent Research and Development in Applied Spectroscopy 1996;1:17-24. 
[440] Berman IR. Handbook of Fluorescence Spectra of Aromatic Molecules. Second ed. New York: Academic Press; 1971.

[441] Singh P, Sung C-J. PAH formation in counterflow non-premixed flames of butane and butanol isomers. Combust Flame 2016;170:91-110.

[442] Bejaoui S, Mercier X, Desgroux P, Therssen E. Laser induced fluorescence spectroscopy of aromatic species produced in atmospheric sooting flames using UV and visible excitation wavelengths. Combust Flame 2014;161:2479-91.

[443] Sirignano M, Conturso M, D'Anna A. Effect of furans on particle formation in diffusion flames: An experimental and modeling study. Proc Combust Inst 2015;35:525-32.

[444] Franzelli B, Scouflaire P, Candel S. Time-resolved spatial patterns and interactions of soot, $\mathrm{PAH}$ and $\mathrm{OH}$ in a turbulent diffusion flame. Proc Combust Inst 2015;35:1921-9.

[445] de Andrade Oliveira MH, Olofsson NE, Johnsson J, Bladh H, Lantz A, Li B, Li ZS, Aldén M, Bengtsson PE, Luijten CCM, de Goey LPH. Soot, PAH and OH measurements in vaporized liquid fuel flames. Fuel 2013;112:145-52.

[446] Verhoeven LM, Oliveira MHD, Lantz A, Li B, Li ZS, Luijten CCM, van Oijen JA, Alden M, de Goey LPH. A numerical and experimental study of Polycyclic Aromatic Hydrocarbons in a laminar diffusion flame. Proc Combust Inst 2013;34:1819-26.

[447] Choi BC, Choi SK, Chung SH. Soot formation characteristics of gasoline surrogate fuels in counterflow diffusion flames. Proc Combust Inst 2011;33:609-16.

[448] Zhang Y, Wang L, Liu P, Li Y, Zhan R, Huang Z, Lin H. Measurement and extrapolation modeling of PAH laser-induced fluorescence spectra at elevated temperatures. Appl Phys B 2018;125:6.

[449] Geigle KP, O'Loughlin W, Hadef R, Meier W. Visualization of soot inception in turbulent pressurized flames by simultaneous measurement of laser-induced fluorescence of polycyclic aromatic hydrocarbons and laser-induced incandescence, and correlation to $\mathrm{OH}$ distributions. Appl Phys B 2015;119:717-30.

[450] Wartel M, Pauwels JF, Desgroux P, Mercier X. Quantitative measurement of naphthalene in low-pressure flames by jet-cooled laser-induced fluorescence. Appl Phys B-Lasers and Optics 2010;100:933-43.

[451] Wartel M, Pauwels JF, Desgroux P, Mercier X. Pyrene measurements in sooting low pressure methane flames by jet-cooled laser-induced fluorescence. J Phys Chem A 2011;115:14153-62. [452] D'Anna A, Commodo M, Violl S, Allouis C, Kent J. Nano organic carbon and soot in turbulent non-premixed ethylene flames. Proc Combust Inst 2007;31:621-9.

[453] Bartos D, Dunn M, Sirignano M, D'Anna A, Masri AR. Tracking the evolution of soot particles and precursors in turbulent flames using laser-induced emission. Proc Combust Inst 2017;36:1869-76.

[454] Commodo M, de Lisio C, D'Anna A, Minutolo P. Size measurements of fluorescent carbon nanoparticles in a coflowing laminar diffusion flame by time-resolved fluorescence anisotropy. Combust Sci Technol 2012;184:916-28.

[455] Bruno A, Ossler F, Minutolo P, de Lisio C. In situ detection of soot nanoparticles by timeresolved fluorescence analysis. J Opt A - Pure Appl Opt 2008;10:5.

[456] Bruno A, Ossler F, de Lisio C, Minutolo P, Spinelli N, D'Alessio A. Detection of fluorescent nanoparticles in flame with femtosecond laser-induced fluorescence anisotropy. Opt Express 2008;16:5623-32.

[457] Goldenstein CS, Miller VA, Hanson RK. Infrared planar laser-induced fluorescence with a $\mathrm{CW}$ quantum-cascade laser for spatially resolved $\mathrm{CO} 2$ and gas properties. Appl Phys B 2015;120:185-99.

[458] Kirby BJ, Hanson RK. Planar laser-induced fluorescence imaging of carbon monoxide using vibrational (infrared) transitions. Appl Phys B 1999;69:505-7. 
[459] Jain A, Wang Y, Kulatilaka WD. Effect of H-atom concentration on soot formation in premixed ethylene/air flames. Proc Combust Inst 2018.

[460] Fuest F, Barlow RS, Chen JY, Dreizler A. Raman/Rayleigh scattering and CO-LIF measurements in laminar and turbulent jet flames of dimethyl ether. Combust Flame 2012;159:2533-62.

[461] Law CK, Sung CJ, Yu G, Axelbaum RL. On the structural sensitivity of purely strained planar premixed flames to strain rate variations. Combust Flame 1994;98:139-54.

[462] Sung CJ, Liu JB, Law CK. Structural response of counterflow diffusion flames to strain rate variations. Combust Flame 1995;102:481-92.

[463] Getty JD, Burmeister MJ, Westre SG, Kelly PB. Examination of allyl radical excited-state dynamics and ground-state vibrational frequencies by ultraviolet resonance Raman spectroscopy. Journal of the American Chemical Society 1991;113:801-5.

[464] Nordström E, Olofsson N-E, Simonsson J, Johnsson J, Bladh H, Bengtsson P-E. Local gas heating in sooting flames by heat transfer from laser-heated particles investigated using rotational CARS and LII. Proc Combust Inst 2015;35:3707-13.

[465] Geigle KP, Schneider-Kühnle Y, Tsurikov MS, Hadef R, Lückerath R, Krüger V, Stricker W, Aigner M. Investigation of laminar pressurized flames for soot model validation using SV-CARS and LII. Proc Combust Inst 2005;30:1645-53.

[466] Dasch CJ. One-dimensional tomography - A comparison of Abel, onion-peeling, and filtered backprojection methods. Appl Optics 1992;31:1146-52.

[467] Daun KJ, Thomson KA, Liu FS, Smallwood GJ. Deconvolution of axisymmetric flame properties using Tikhonov regularization. Appl Optics 2006;45:4638-46.

[468] Farias TL, Carvalho MG, Koylu UO, Faeth GM. Computational evaluation of approximate Rayleigh-Debye-Gans/Fractal-Aggregate theory for the absorption and scattering properties of soot. J Heat Trans-T ASME 1995;117:152-9.

[469] Choi MY, Mulholland GW, Hamins A, Kashiwagi T. Comparisons of the soot volume fraction using gravimetric and light extinction techniques. Combust Flame 1995;102:161-9. [470] Mountain RD, Mulholland GW. Light scattering from simulated smoke agglomerates. Langmuir 1988;4:1321-6.

[471] Simonsson J, Olofsson NE, Torok S, Bengtsson PE, Bladh H. Wavelength dependence of extinction in sooting flat premixed flames in the visible and near-infrared regimes. Appl Phys B-

Lasers and Optics 2015;119:657-67.

[472] Moreau CS, Therssen E, Mercier X, Pauwels JF, Desgroux P. Two-color laser-induced incandescence and cavity ring-down spectroscopy for sensitive and quantitative imaging of soot and PAHs in flames. Appl Phys B-Lasers and Optics 2004;78:485-92.

[473] Zerbs J, Geigle KP, Lammel O, Hader J, Stirn R, Hadef R, Meier W. The influence of wavelength in extinction measurements and beam steering in laser-induced incandescence measurements in sooting flames. Appl Phys B 2009;96:683-94.

[474] Michelsen HA, Schrader PE, Goulay F. Wavelength and temperature dependences of the absorption and scattering cross sections of soot. Carbon 2010;48:2175-91.

[475] Cleon G, Amodeo T, Faccinetto A, Desgroux P. Laser induced incandescence determination of the ratio of the soot absorption functions at $532 \mathrm{~nm}$ and $1064 \mathrm{~nm}$ in the nucleation zone of a low pressure premixed sooting flame. Appl Phys B-Lasers and Optics 2011;104:297-305.

[476] Choi JH, Choi BC, Lee SM, Chung SH, Jung KS, Jeong WL, Choi SK, Park SK. Effects of DME mixing on number density and size properties of soot particles in counterflow non-premixed ethylene flames. J Mech Sci Technol 2015;29:2259-67.

[477] Thomson KA, Johnson MR, Snelling DR, Smallwood GJ. Diffuse-light two-dimensional lineof-sight attenuation for soot concentration measurements. Appl Optics 2008;47:694-703.

[478] Kashif M, Guibert P, Bonnety J, Legros G. Sooting tendencies of primary reference fuels in atmospheric laminar diffusion flames burning into vitiated air. Combust Flame 2014;161:1575-86. 
[479] Kashif M, Bonnety J, Guibert P, Morin C, Legros G. Soot volume fraction fields in unsteady axis-symmetric flames by continuous laser extinction technique. Opt Express 2012;20:28742-51. [480] Snelling DR, Thomson KA, Smallwood GJ, Oslash, G-uacute L, lder, Weckman EJ, Fraser RA. Spectrally resolved measurement of flame radiation to determine soot temperature and concentration. AIAA J 2002;40:1789-95.

[481] Hall RJ, Bonczyk PA. Sooting flame thermometry using emission absorption tomography. Appl Optics 1990;29:4590-8.

[482] Liu F, Thomson KA, Smallwood GJ. Numerical investigation of the effect of signal trapping on soot measurements using LII in laminar coflow diffusion flames. Appl Phys B 2009;96:671-82. [483] Thomson KA, Gülder ÖL, Weckman EJ, Fraser RA, Smallwood GJ, Snelling DR. Soot concentration and temperature measurements in co-annular, nonpremixed $\mathrm{CH} 4$ /air laminar flames at pressures up to $4 \mathrm{MPa}$. Combust Flame 2005;140:222-32.

[484] Liu F, Thomson KA, Smallwood GJ. Soot temperature and volume fraction retrieval from spectrally resolved flame emission measurement in laminar axisymmetric coflow diffusion flames: Effect of self-absorption. Combust Flame 2013;160:1693-705.

[485] Kholghy MR, Afarin Y, Sediako AD, Barba J, Lapuerta M, Chu C, Weingarten J, Borshanpour B, Chernov V, Thomson MJ. Comparison of multiple diagnostic techniques to study soot formation and morphology in a diffusion flame. Combust Flame 2017;176:567-83.

[486] Karataş AE, Intasopa G, Gülder ÖL. Sooting behaviour of n-heptane laminar diffusion flames at high pressures. Combust Flame 2013;160:1650-6.

[487] Yelverton TLB, Roberts WL. Soot surface temperature measurements in pure and diluted flames at atmospheric and elevated pressures. Exp Therm Fluid Sci 2008;33:17-22.

[488] Thomsen MC, Fuentes A, Demarco R, Volkwein C, Consalvi JL, Reszka P. Soot

measurements in candle flames. Experimental Thermal and Fluid Science 2017;82:116-23.

[489] Gleason K, Carbone F, Gomez A. Effect of temperature on soot inception in highly controlled counterflow ethylene diffusion flames. Combust Flame 2018;192:283-94.

[490] Gleason K, Carbone F, Gomez A. Pressure and temperature dependence of soot in highly controlled counterflow ethylene diffusion flames. Proc Combust Inst 2019;37:2057-64.

[491] Schulz C, Kock BF, Hofmann M, Michelsen H, Will S, Bougie B, Suntz R, Smallwood G. Laser-induced incandescence: Recent trends and current questions. Appl Phys B-Lasers and Optics 2006;83:333-54.

[492] Melton LA. Soot Diagnostics Based on Laser-Heating. Appl Optics 1984;23:2201-8.

[493] Bladh H, Johnsson J, Bengtsson PE. On the dependence of the laser-induced incandescence

(LII) signal on soot volume fraction for variations in particle size. Appl Phys B 2008;90:109-25.

[494] Vanderwal RL, Weiland KJ. Laser-induced incandescence - Development and

characterization towards a measurement of soot-volume fraction. Appl Phys B-Lasers and Optics 1994;59:445-52.

[495] Snelling DR, Smallwood GJ, Liu F, Gülder ÖL, Bachalo WD. A calibration-independent laser-induced incandescence technique for soot measurement by detecting absolute light intensity. Appl Optics 2005;44:6773-85.

[496] De Luliis S, Cignoli F, Zizak G. Two-color laser-induced incandescence (2C-LII) technique for absolute soot volume fraction measurements in flames. Appl Optics 2005;44:7414-23.

[497] Vander Wal RL, Ticich TM, Stephens AB. Can soot primary particle size be determined using laser-induced incandescence? Combust Flame 1999;116:291-6.

[498] Michelsen HA, Liu F, Kock BF, Bladh H, Boiarciuc A, Charwath M, Dreier T, Hadef R, Hofmann M, Reimann J, Will S, Bengtsson PE, Bockhorn H, Foucher F, Geigle KP, MounaïmRousselle C, Schulz C, Stirn R, Tribalet B, Suntz R. Modeling laser-induced incandescence of soot: a summary and comparison of LII models. Appl Phys B 2007;87:503-21.

[499] De Iuliis S, Migliorini F, Cignoli F, Zizak G. Peak soot temperature in laser-induced incandescence measurements. Appl Phys B-Lasers and Optics 2006;83:397-402. 
[500] Bladh H, Johnsson J, Olofsson NE, Bohlin A, Bengtsson PE. Optical soot characterization using two-color laser-induced incandescence (2C-LII) in the soot growth region of a premixed flat flame. Proc Combust Inst 2011;33:641-8.

[501] Oh KC, Shin HD. The effect of oxygen and carbon dioxide concentration on soot formation in nonpremixed flames using time resolved LII technique. J Mech Sci Technol 2005;19:2068-76. [502] Sun ZW, Gu DH, Nathan GJ, Alwahabi ZT, Dally BB. Single-shot, time-resolved planar Laser-Induced incandescence (TiRe-LII) for soot primary particle sizing in flames. Proc Combust Inst 2015;35:3673-80.

[503] Gu D, Sun Z, Dally BB, Medwell PR, Alwahabi ZT, Nathan GJ. Simultaneous measurements of gas temperature, soot volume fraction and primary particle diameter in a sooting lifted turbulent ethylene/air non-premixed flame. Combust Flame 2017;179:33-50.

[504] Koylu UO, McEnally CS, Rosner DE, Pfefferle LD. Simultaneous measurements of soot volume fraction and particle size/microstructure in flames using a thermophoretic sampling technique. Combust Flame 1997;110:494-507.

[505] Zhao B, Uchikawa K, Wang H. A comparative study of nanoparticles in premixed flames by scanning mobility particle sizer, small angle neutron scattering, and transmission electron microscopy. Proc Combust Inst 2007;31:851-60.

[506] Merchan-Merchan W, Saveliev A, Kennedy LA, Fridman A. Formation of carbon nanotubes in counter-flow, oxy-methane diffusion flames without catalysts. Chem Phys Lett 2002;354:20-4.

[507] Lee J, Altman I, Choi M. Design of thermophoretic probe for precise particle sampling. J Aerosol Sci 2008;39:418-31.

[508] Figura L. Experimental study of incipiently-sooting counterflow diffusion flames at high pressures: Yale University; 2014.

[509] Vargas AM, Gülder ÖL. A multi-probe thermophoretic soot sampling system for highpressure diffusion flames. Rev Sci Instrum 2016;87:055101.

[510] Yehliu K, Vander Wal RL, Boehman AL. Development of an HRTEM image analysis

method to quantify carbon nanostructure. Combust Flame 2011;158:1837-51.

[511] Schenk M, Lieb S, Vieker H, Beyer A, Gölzhäuser A, Wang H, Kohse-Höinghaus K. Imaging Nanocarbon Materials: Soot Particles in Flames are Not Structurally Homogeneous.

ChemPhysChem 2013;14:3248-54.

[512] Wan K, Chen D, Wang H. On imaging nascent soot by transmission electron microscopy. Combust Flame 2018;198:260-6.

[513] Commodo M, De Falco G, Minutolo P, D'Anna A. Structure and size of soot nanoparticles in laminar premixed flames at different equivalence ratios. Fuel 2018;216:456-62.

[514] Lin B, Gu H, Ni H, Guan B, Li Z, Han D, Gu C, Shao C, Huang Z, Lin H. Effect of mixing methane, ethane, propane and ethylene on the soot particle size distribution in a premixed propene flame. Combust Flame 2018;193:54-60.

[515] Botero ML, Mosbach S, Kraft M. Sooting tendency of paraffin components of diesel and gasoline in diffusion flames. Fuel 2014;126:8-15.

[516] Li Z, Wang H. Drag force, diffusion coefficient, and electric mobility of small particles. I. Theory applicable to the free-molecule regime. Phys Rev E 2003;68:061206.

[517] Li Z, Wang H. Drag force, diffusion coefficient, and electric mobility of small particles. II. Application. Phys Rev E 2003;68:061207.

[518] Li Z, Wang H. Comment on "Phenomenological description of mobility of nm- and sub-nmsized charged aerosol particles in electric field" by Shandakov, S. D., Nasibulin, A. G. and Kauppinen, E. I. J Aerosol Sci 2006;37:111-4.

[519] McMurry PH. The History of Condensation Nucleus Counters. Aerosol Sci Tech 2000;33:297-322.

[520] Lehtipalo K. How the development of condensed particle counters is reforming our view on atmospheric nucleation. Helsinki, Finland: University of Helsinki; 2011. 
[521] Iida K, Stolzenburg MR, McMurry PH. Effect of Working Fluid on Sub-2 nm Particle Detection with a Laminar Flow Ultrafine Condensation Particle Counter. Aerosol Sci Tech 2009;43:81-96.

[522] Jiang J, Chen M, Kuang C, Attoui M, McMurry PH. Electrical Mobility Spectrometer Using a Diethylene Glycol Condensation Particle Counter for Measurement of Aerosol Size Distributions Down to $1 \mathrm{~nm}$. Aerosol Sci Tech 2011;45:510-21.

[523] de la Mora JF. Heterogeneous Nucleation with Finite Activation Energy and Perfect Wetting: Capillary Theory Versus Experiments with Nanometer Particles, and Extrapolations on the Smallest Detectable Nucleus. Aerosol Sci Tech 2011;45:543-54.

[524] Kohse-Höinghaus K, Jeffries JB. Applied combustion diagnostics. London: Taylor and Francis; 2002.

[525] Zhang C, Atreya A, Lee K. Sooting structure of methane counterflow diffusion flames with preheated reactants and dilution by products of combustion. Proc Combust Inst 1992;24:1049-57. [526] Olten N, Senkan SM. On-line measurements of the polycyclic aromatic hydrocarbons (PAH) in counter-flow ethylene diffusion flame. Combust Sci Technol 2000;159:1-15.

[527] Park DG, Choi BC, Cha MS, Chung SH. Soot reduction under DC electric fields in counterflow non-premixed laminar ethylene flames. Combust Sci Technol 2014;186:644-56. [528] Hwang JY, Lee W, Kang HG, Chung SH. Synergistic effect of ethylene-propane mixture on soot formation in laminar diffusion flames. Combust Flame 1998;114:370-80.

[529] Amin HMF, Roberts WL. Soot measurements by two angle scattering and extinction in an $\mathrm{N}_{2}$ diluted ethylene/air counterflow diffusion flame from 2 to 5 atm. Proc Combust Inst 2017;36:861-9. [530] Sarnacki BG, Chelliah HK. Sooting limits of non-premixed counterflow ethylene/oxygen/inert flames using LII: Effects of flow strain rate and pressure (up to $30 \mathrm{~atm}$ ). Combust Flame 2018.

[531] D’Anna A, Commodo M, Sirignano M, Minutolo P, Pagliara R. Particle formation in opposed-flow diffusion flames of ethylene: An experimental and numerical study. Proc Combust Inst 2009;32:793-801.

[532] Karataş AE, Gülder ÖL. Dependence of sooting characteristics and temperature field of coflow laminar pure and nitrogen-diluted ethylene-air diffusion flames on pressure. Combust Flame 2015;162:1566-74.

[533] Joo HI, Gülder ÖL. Soot formation and temperature field structure in co-flow laminar methane-air diffusion flames at pressures from 10 to 60atm. Proc Combust Inst 2009;32:769-75. [534] Wang Q, Legros G, Bonnety J, Morin C. Experimental characterization of the different nitrogen dilution effects on soot formation in ethylene diffusion flames. Proc Combust Inst 2017;36:3227-35.

[535] Mikofski MA, Williams TC, Shaddix CR, Fernandez-Pello AC, Blevins LG. Structure of laminar sooting inverse diffusion flames. Combust Flame 2007;149:463-78.

[536] Makel DB, Kennedy IM. Soot formation in laminar inverse diffusion flames. Combust Sci Technol 1994;97:303-14.

[537] Bisetti F, Abdelgadir A, Steinmetz SA, Attili A, Roberts WL. Self-similar scaling of pressurised sooting methane/air coflow flames at constant Reynolds and Grashof numbers. Combust Flame 2018;196:300-13.

[538] Wang Z, Sunderland PB, Axelbaum RL. Dilution effects on laminar jet diffusion flame lengths. Proc Combust Inst 2019;37:1547-53.

[539] Atreya A, Zhang C, Kim HK, Shamim T, Suh J. The effect of changes in the flame structure on the formation and destruction of soot and NOx in radiating diffusion flames. Proc Combust Inst 1996;26:2181-9.

[540] Du J, Axelbaum RL. The effect of flame structure on soot-particle inception in diffusion flames. Combust Flame 1995;100:367-75. 
[541] Kitajima A, Hatanaka T, Takeuchi M, Torikai H, Miyadera T. Experimental study of PAH formation in laminar counterflow $\mathrm{CH}_{4}-\mathrm{N}_{2} / \mathrm{O}_{2}-\mathrm{N}_{2}$ and $\mathrm{C}_{3} \mathrm{H}_{8}-\mathrm{N}_{2} / \mathrm{O}_{2}-\mathrm{N}_{2}$ nonpremixed flames. Combust Flame 2005;142:72-88.

[542] Lin KC, Faeth GM. Effects of hydrodynamics on soot formation in laminar opposed-jet diffusion flames. Journal of Propulsion and Power 1996;12:691-8.

[543] Skeen SA, Yablonsky G, Axelbaum RL. Characteristics of non-premixed oxygen-enhanced combustion: II. Flame structure effects on soot precursor kinetics resulting in soot-free flames.

Combust Flame 2010;157:1745-52.

[544] Chao BH, Liu S, Axelbaum RL. On soot inception in nonpremixed flames and the effects of flame structure. Combust Sci Technol 1998;138:105-35.

[545] Guo Z, Lou C, Liu Z, Zhou H. The impact of combustion characteristics and flame structure on soot formation in oxy-enhanced and oxy-fuel diffusion flames. Sci China-Technol Sci

2013;56:1618-28.

[546] Skeen SA, Yablonsky G, Axelbaum RL. Characteristics of non-premixed oxygen-enhanced combustion: I. The presence of appreciable oxygen at the location of maximum temperature.

Combust Flame 2009; 156:2145-52.

[547] Sun CJ, Sung CJ, Wang H, Law CK. On the structure of nonsooting counterflow ethylene and acetylene diffusion flames. Combust Flame 1996;107:321-35.

[548] Xia F, Axelbaum RL. Simplifying the complexity of diffusion flames through interpretation in C/O ratio space. Comput Math Appl 2013;65:1625-32.

[549] Chung SH, Law CK. An invariant derivation of flame stretch. Combust Flame 1984;55:123-5. [550] Niemann U, Seshadri K, Williams FA. Accuracies of laminar counterflow flame experiments. Combust Flame 2015;162:1540-9.

[551] Du DX, Axelbaum RL, Law CK. Experiments on the sooting limits of aerodynamicallystrained diffusion flames. Proc Combust Inst 1989;22:387-94.

[552] Seshadri K, Williams FA. Laminar flow between parallel plates with injection of a reactant at high reynolds number. Int J Heat Mass Tran 1978;21:251-3.

[553] Chelliah HK, Law CK, Ueda T, Smooke MD, Williams FA. An experimental and theoretical investigation of the dilution, pressure and flow-field effects on the extinction condition of methaneair-nitrogen diffusion flames. Proc Combust Inst 1991;23:503-11.

[554] Vandsburger U, Kennedy IM, Glassman I. Sooting counter-flow diffusion flames with varying velocity gradients. Proc Combust Inst 1985;20:1105-12.

[555] Williams FA. Progress in knowledge of flamelet structure and extinction. Prog Energy Combust Sci 2000;26:657-82.

[556] Sohrab SH, Ye ZY, Law CK. An experimental investigation on flame interaction and the existence of negative flame speeds. Proc Combust Inst 1985;20:1957-65.

[557] Chung SH, Kim JS, Law CK. Extinction of interacting premixed flames: Theory and experimental comparisons. Proc Combust Inst 1988;21:1845-51.

[558] Law CK, L Zhu D, Li TX, Chung SH, Kim JS. On the structure and extinction dynamics of partially-premixed flames: Theory and experiment. Combust Sci Technol 1989;64:199-232.

[559] Chung SH. Interaction of Flames. In: Cheremisinoff NP, editor. Handbook of Heat and Mass Transfer: Gulf Publ. Co.; 1990. p. 863-91.

[560] Mungekar H, Atreya A. NO formation in counterflow partially premixed flames. Combust Flame 2007; 148:148-57.

[561] Mungekar HP, Atreya A. Effect of partial premixing on the sooting structure of methane flames. Combust Flame 2006;144:336-48.

[562] Mungekar HP, Atreya A. Flame radiation and soot emission from partially premixed methane counterflow flames. J Heat Trans-T ASME 2006;128:361-7. 
[563] Berta P, Aggarwal SK, Puri IK. An experimental and numerical investigation of n-heptane/air counterflow partially premixed flames and emission of NOx and PAH species. Combust Flame 2006; 145:740-64.

[564] Du DX, Axelbaum RL, Law CK. The influence of carbon dioxide and oxygen as additives on soot formation in diffusion flames. Proc Combust Inst 1991;23:1501-7.

[565] Baranov AF, Prisnyakov VF, Taran EN. Early soot formation stages in low-pressure diffusional counterflow hydrocarbon flames. Combust Explosion Shock Waves 1993;29:345-7.

[566] Taran ÉN. Effect of an alternating electric field on soot formation in diffusional low-pressure counterflow hydrocarbon flames. Combust Explosion Shock Waves 1993;29:348-52.

[567] Delichatsios MA. A phenomenological model for smoke-point and soot formation in laminar flames. Combust Sci Technol 1994;100:283-98.

[568] Du DX, Axelbaum RL, Law CK. Soot formation in strained diffusion flames with gaseous additives. Combust Flame 1995;102:11-20.

[569] Yang GS, Kennedy IM. Soot measurements in a chlorinated counterflow diffusion flame using a laser scattering and extinction technique. KSME J 1995;9:369-76.

[570] Tseng L-K, Gore JP, Puri IK, Takeno T. Acetylene and ethylene mole fractions in methane/air partially premixed flames. Proc Combust Inst 1996;26:993-9.

[571] Marquardt M, Mauss F, Jungfleisch B, Suntz R, Bockhorn H. Re-initiation of soot surface growth in premixed counterflow flames. Proc Combust Inst 1996:2343-50.

[572] Vincitore AM, Senkan SM. Experimental studies of the micro-structures of opposed flow diffusion flames: Methane. Combust Sci Technol 1997;130:233-46.

[573] Du DX, Wang H, Law CK. Soot formation in counterflow ethylene diffusion flames from 1 to 2.5 atmospheres. Combust Flame 1998;113:264-70.

[574] Rubino L, Thomson MJ. The effect of oxygenated additives on soot precursor formation in a counterflow diffusion flame. No. 1999-01-3589, SAE International; 1999.

[575] Bohm H, Lacas F. On extinction limits and polycyclic aromatic hydrocarbon formation in strained counterflow diffusion flames from 1 to 6 bar. Proc Combust Inst 2000;28:2627-34.

[576] Beltrame A, Porshnev P, Merchan-Merchan W, Saveliev A, Fridman A, Kennedy LA, Petrova O, Zhdanok S, Amouri F, Charon O. Soot and NO formation in methane-oxygen enriched diffusion flames. Combust Flame 2001;124:295-310.

[577] Santoianni DA, DeCroix ME, Roberts WL. Temperature imaging in an unsteady propane-air counterflow diffusion flame subjected to low frequency oscillations. Flow Turbulence and Combustion 2001;66:23-36.

[578] Granata S, Faravelli T, Ranzi E, Olten N, Senkan S. Kinetic modeling of counterflow diffusion flames of butadiene. Combust Flame 2002;131:273-84.

[579] Naik SV, Laurendeau NM. Laser-saturated and linear laser-induced fluorescence measurements of nitric oxide in counterflow diffusion flames under non-sooting oxygen-enriched conditions. Combust Sci Technol 2002;174:1-21.

[580] Pels Leusden C, Hasse C, Peters N. Aggregate formation in sooting counterflow diffusion flames. Proc Combust Inst 2002;29:2383-90.

[581] Naik SV, Laurendeau NM, Cooke JA, Smooke MD. A soot map for methane-oxygen counterflow diffusion flames. Combust Sci Technol 2003;175:1165-77.

[582] Lee SM, Yoon SS, Chung SH. Synergistic effect on soot formation in counterflow diffusion flames of ethylene-propane mixtures with benzene addition. Combust Flame 2004;136:493-500. [583] Liu FS, Guo HS, Smallwood GJ, El Hafi M. Effects of gas and soot radiation on soot formation in counterflow ethylene diffusion flames. J Quant Spectrosc Ra 2004;84:501-11. [584] Berta P, Puri IK, Aggarwal SK. Structure of partially premixed n-heptane-air counterflow flames. Proc Combust Inst 2005;30:447-53.

[585] Xiao J, Austin E, Roberts WL. Relative polycyclic aromatic hydrocarbon concentrations in unsteady counterflow diffusion flames. Combust Sci Technol 2005;177:691-713. 
[586] Yoon SS, Lee SM, Chung SH. Effect of mixing methane, ethane, propane, and propene on the synergistic effect of PAH and soot formation in ethylene-base counterflow diffusion flames. Proc Combust Inst 2005;30:1417-24.

[587] Humer S, Frassoldati A, Granata S, Faravelli T, Ranzi E, Seiser R, Seshadri K. Experimental and kinetic modeling study of combustion of JP-8, its surrogates and reference components in laminar nonpremixed flows. Proc Combust Inst 2007;31:393-400.

[588] Li TX, Zhang HG, Wang FJ, Chen Z, Saito K. Synthesis of carbon nanotubes on Ni-alloy and Si-substrates using counterflow methane-air diffusion flames. Proc Combust Inst 2007;31:1849-56. [589] Sarathy SM, Gail S, Syed SA, Thomson MJ, Dagaut P. A comparison of saturated and unsaturated C-4 fatty acid methyl esters in an opposed flow diffusion flame and a jet stirred reactor. Proc Combust Inst 2007;31:1015-22.

[590] Yamamoto M, Duan S, Senkan S. The effect of strain rate on polycyclic aromatic hydrocarbon (PAH) formation in acetylene diffusion flames. Combust Flame 2007;151:532-41. [591] Yoo CS, Im HG. Transient soot dynamics in turbulent nonpremixed ethylene-air counterflow flames. Proc Combust Inst 2007;31:701-8.

[592] Berta P, Aggarwal SK, Puri IK, Granata S, Faravelli T, Ranzi E. Experimental and numerical investigation of n-heptane/air counterflow nonpremixed flame structure. Journal of Propulsion and Power 2008;24:797-804.

[593] Cuoci A, Frassoldati A, Faravelli T, Ranzi E. Frequency response of counter flow diffusion flames to strain rate harmonic oscillations. Combust Sci Technol 2008;180:767-84.

[594] Leylegian JC. Soot formation in aerodynamically strained methane-air and ethylene-air diffusion flames with chloromethane addition. Combust Flame 2008;152:144-53.

[595] Zhong B, Xi J. Reduced kinetic mechanism of n-heptane oxidation in modeling polycyclic aromatic hydrocarbon formation in opposed-flow diffusion flames. Frontiers of Energy and Power Engineering in China 2008;2:326-32.

[596] Blanquart G, Pepiot-Desjardins P, Pitsch H. Chemical mechanism for high temperature combustion of engine relevant fuels with emphasis on soot precursors. Combust Flame 2009;156:588-607.

[597] Blanquart G, Pitsch $\mathrm{H}$. Analyzing the effects of temperature on soot formation with a joint volume-surface-hydrogen model. Combust Flame 2009;156:1614-26.

[598] Cuoci A, Frassoldati A, Faravelli T, Ranzi E. Soot formation in unsteady counterflow diffusion flames. Proc Combust Inst 2009;32:1335-42.

[599] Hou S-S, Chung D-H, Lin T-H. High-yield synthesis of carbon nano-onions in counterflow diffusion flames. Carbon 2009;47:938-47.

[600] Mehta RS, Haworth DC, Modest MF. An assessment of gas-phase reaction mechanisms and soot models for laminar atmospheric-pressure ethylene-air flames. Proc Combust Inst 2009;32:1327-34.

[601] Frassoldati A, Cuoci A, Faravelli T, Niemann U, Ranzi E, Seiser R, Seshadri K. An experimental and kinetic modeling study of n-propanol and iso-propanol combustion. Combust Flame 2010;157:2-16.

[602] Choi BC, Choi SK, Chung SH, Kim JS, Choi JH. Experimental and numerical investigation of fuel mixing effects on soot structures in counterflow diffusion flames. International Journal of Automotive Technology 2011;12:183-91.

[603] Hayashi J, Watanabe H, Kurose R, Akamatsu F. Effects of fuel droplet size on soot formation in spray flames formed in a laminar counterflow. Combust Flame 2011;158:2559-68.

[604] Narayanan P, Baum HR, Trouve A. Effect of soot addition on extinction limits of luminous laminar counterflow diffusion flames. Proc Combust Inst 2011;33:2539-46.

[605] Seiser R, Niemann U, Seshadri K. Experimental study of combustion of n-decane and JP-10 in non-premixed flows. Proc Combust Inst 2011;33:1045-52. 
[606] Carbone F, Gomez A. The structure of toluene-doped counterflow gaseous diffusion flames. Combust Flame 2012;159:3040-55.

[607] Salamanca M, Sirignano M, D'Anna A. Particulate Formation in Premixed and Counter-flow Diffusion Ethylene/Ethanol Flames. Energ Fuel 2012;26:6144-52.

[608] Sirignano M, Collina A, Commodo M, Minutolo P, D'Anna A. Detection of aromatic hydrocarbons and incipient particles in an opposed-flow flame of ethylene by spectral and timeresolved laser induced emission spectroscopy. Combust Flame 2012;159:1663-9.

[609] Carbone F, Gomez A. Chemical effects of 1,2,4-trimethyl benzene addition in counterflow gaseous diffusion flames. Proc Combust Inst 2013;34:1025-33.

[610] Fu X, Han X, Brezinsky K, Aggarwal S. Effect of fuel molecular structure and premixing on soot emissions from n-heptane and 1-heptene flames. Energ Fuel 2013;27:6262-72.

[611] Han X, Aggarwal SK, Brezinsky K. Effect of unsaturated bond on NOx and PAH formation in n-heptane and 1-heptene triple flames. Energ Fuel 2013;27:537-48.

[612] Hayashi J, Fukui J, Akamatsu F. Effects of fuel droplet size distribution on soot formation in spray flames formed in a laminar counterflow. Proc Combust Inst 2013;34:1561-8.

[613] Hernandez I, Lecocq G, Poitou D, Riber E, Cuenot B. Computations of soot formation in ethylene/air counterflow diffusion flames and its interaction with radiation. Comptes Rendus Mecanique 2013;341:238-46.

[614] Joo PH, Wang Y, Raj A, Chung SH. Sooting limit in counterflow diffusion flames of ethylene/propane fuels and implication to threshold soot index. Proc Combust Inst 2013;34:1803-9. [615] Skeen SA, Michelsen HA, Wilson KR, Popolan DM, Violi A, Hansen N. Near-threshold photoionization mass spectra of combustion-generated high-molecular-weight soot precursors. J Aerosol Sci 2013;58:86-102.

[616] Wang Y, Raj A, Chung SH. A PAH growth mechanism and synergistic effect on PAH formation in counterflow diffusion flames. Combust Flame 2013;160:1667-76.

[617] Carbone F, Gomez A. Experimental study on the structure of opposed flow gaseous diffusion flames doped with n-decane. Combust Flame 2014;161:453-64.

[618] Figura L, Gomez A. Structure of incipiently sooting ethylene-nitrogen counterflow diffusion flames at high pressures. Combust Flame 2014;161:1587-603.

[619] Sirignano M, Salamanca M, D'Anna A. The role of dimethyl ether as substituent to ethylene on particulate formation in premixed and counter-flow diffusion flames. Fuel 2014;126:256-62.

[620] Wang Y, Chung SH. Effect of strain rate on sooting limits in counterflow diffusion flames of gaseous hydrocarbon fuels: Sooting temperature index and sooting sensitivity index. Combust Flame 2014;161:1224-34.

[621] Carbone F, Gomez A. Chemical interactions between 1,2,4-trimethylbenzene and n-decane in doped counterflow gaseous diffusion flames. Proc Combust Inst 2015;35:761-9.

[622] Choi SK, Choi BC, Lee SM, Choi JH. The effect of liquid fuel doping on PAH and soot formation in counterflow ethylene diffusion flames. Exp Therm Fluid Sci 2015;60:123-31.

[623] Figura L, Carbone F, Gomez A. Challenges and artifacts of probing high-pressure counterflow laminar diffusion flames. Proc Combust Inst 2015;35:1871-8.

[624] Choi J-H, Hwang C-H, Choi SK, Lee SM, Lee WJ, Jang SH, Park S-H. Impacts of hydrogen addition on micro and nanostructure of soot particles formed in $\mathrm{C} 2 \mathrm{H} 4$ /air counter diffusion flames. International Journal of Hydrogen Energy 2016;41:15852-8.

[625] Selvaraj P, Arias PG, Lee BJ, Im HG, Wang Y, Gao Y, Park S, Sarathy SM, Lu T, Chung SH. A computational study of ethylene-air sooting flames: Effects of large polycyclic aromatic hydrocarbons. Combust Flame 2016;163:427-36.

[626] Wang Y, Chung SH. Formation of soot in counterflow diffusion flames with carbon dioxide dilution. Combust Sci Technol 2016;188:805-17. 
[627] Zimmer L, Pereira FM, van Oijen JA, de Goey LPH. Investigation of mass and energy coupling between soot particles and gas species in modelling ethylene counterflow diffusion flames. Combust Theor Model 2017;21:358-79.

[628] Carbone F, Gleason K, Gomez A. Pressure effects on incipiently sooting partially premixed counterflow flames of ethylene. Proc Combust Inst 2017;36:1395-402.

[629] Park S, Wang Y, Chung SH, Sarathy SM. Compositional effects on PAH and soot formation in counterflow diffusion flames of gasoline surrogate fuels. Combust Flame 2017;178:46-60.

[630] Rodrigues P, Franzelli B, Vicquelin R, Gicquel O, Darabiha N. Unsteady dynamics of PAH and soot particles in laminar counterflow diffusion flames. Proc Combust Inst 2017;36:927-34.

[631] Xue X, Hui X, Singh P, Sung C-J. Soot formation in non-premixed counterflow flames of conventional and alternative jet fuels. Fuel 2017;210:343-51.

[632] Amin HMF, Bennett A, Roberts WL. Determining fractal properties of soot aggregates and primary particle size distribution in counterflow flames up to $10 \mathrm{~atm}$. Proc Combust Inst 2018. [633] Li Z, Amin HMF, Liu P, Wang Y, Chung SH, Roberts WL. Effect of dimethyl ether (DME) addition on sooting limits in counterflow diffusion flames of ethylene at elevated pressures. Combust Flame 2018;197:463-70.

[634] Raj A, Tan Z, Zhu D, Croiset E, Wen JZ. On the particle evolution in iron pentacarbonyl loaded counterflow methane-air flame. Combust Flame 2018;194:1-14.

[635] Wang Y, Park S, Sarathy SM, Chung SH. A comparative study on the sooting tendencies of various 1-alkene fuels in counterflow diffusion flames. Combust Flame 2018;192:71-85.

[636] Xue X, Singh P, Sung C-J. Soot formation in counterflow non-premixed ethylene flames at elevated pressures. Combust Flame 2018.

[637] Xue X, Hui X, Vannorsdall P, Singh P, Sung C-J. The blending effect on the sooting tendencies of alternative/conventional jet fuel blends in non-premixed flames. Fuel 2019;237:64857.

[638] Yan F, Zhou M, Xu L, Wang Y, Chung SH. An experimental study on the spectral dependence of light extinction in sooting ethylene counterflow diffusion flames. Exp Therm Fluid Sci 2019;100:259-70.

[639] Hoerlle CA, Pereira FM. Effects of CO2 addition on soot formation of ethylene non-premixed flames under oxygen enriched atmospheres. Combust Flame 2019;203:407-23.

[640] Mahmoud NM, Yan F, Zhou M, Xu L, Wang Y. Coupled Effects of Carbon Dioxide and Water Vapor Addition on Soot Formation in Ethylene Diffusion Flames. Energ Fuel 2019.

[641] Olson DB, Pickens JC. The effects of molecular-structure on soot formation .1. Soot thresholds in premixed flames. Combust Flame 1984;57:199-208.

[642] McEnally CS, Pfefferle LD. The effects of dimethyl ether and ethanol on benzene and soot formation in ethylene nonpremixed flames. Proc Combust Inst 2007;31:603-10.

[643] Ying Y, Liu D. Effects of butanol isomers additions on soot nanostructure and reactivity in normal and inverse ethylene diffusion flames. Fuel 2017;205:109-29.

[644] Brezinsky K, Hura HS, Glassman I. Oxidation pyrolysis chemistry as related to fuel sooting tendencies. Energ Fuel 1988;2:487-93.

[645] Calcote HF, Manos DM. Effect of molecular structure on incipient soot formation. Combust Flame 1983;49:289-304.

[646] McEnally CS, Pfefferle LD. Fuel decomposition and hydrocarbon growth processes for oxygenated hydrocarbons: butyl alcohols. Proc Combust Inst 2005;30:1363-70.

[647] Sundaram KM, Froment GF. Modeling of thermal cracking kinetics-II: Cracking of isobutane, of n-butane and of mixtures ethane-propane-n-butane. Chemical Engineering Science 1977;32:609-17.

[648] Merchant SS, Zanoelo EF, Speth RL, Harper MR, Van Geem KM, Green WH. Combustion and pyrolysis of iso-butanol: Experimental and chemical kinetic modeling study. Combust Flame 2013;160:1907-29. 
[649] Wang H, You X, Joshi A, Davis S, Laskin A, Egolfopoulos FN, Law CK. USC Mech Version II. http://ignis.usc.edu/USC Mech II.htm 2007.

[650] Sarathy SM, Kukkadapu G, Mehl M, Javed T, Ahmed A, Naser N, Tekawade A, Kosiba G, AlAbbad M, Singh E, Park S, Rashidi MA, Chung SH, Roberts WL, Oehlschlaeger MA, Sung C-J, Farooq A. Compositional effects on the ignition of FACE gasolines. Combust Flame 2016;169:17193.

[651] Mehl M, Pitz WJ, Westbrook CK, Curran HJ. Kinetic modeling of gasoline surrogate components and mixtures under engine conditions. Proc Combust Inst 2011;33:193-200.

[652] Kikui S, Nakamura H, Tezuka T, Hasegawa S, Maruta K. Study on combustion and ignition characteristics of ethylene, propylene, 1-butene and 1-pentene in a micro flow reactor with a controlled temperature profile. Combust Flame 2016;163:209-19.

[653] McEnally CS, Pfefferle LD. Sooting tendencies of oxygenated hydrocarbons in laboratoryscale flames. Environ Sci Technol 2011;45:2498-503.

[654] Jin HF, Wang YZ, Zhang KW, Guo HS, Qi F. An experimental study on the formation of polycyclic aromatic hydrocarbons in laminar coflow non-premixed methane/air flames doped with four isomeric butanols. Proc Combust Inst 2013;34:779-86.

[655] Gaïl S, Sarathy SM, Thomson MJ, Diévart P, Dagaut P. Experimental and chemical kinetic modeling study of small methyl esters oxidation: Methyl (E)-2-butenoate and methyl butanoate.

Combust Flame 2008;155:635-50.

[656] Sarathy SM, Kukkadapu G, Mehl M, Wang W, Javed T, Park S, Oehlschlaeger MA, Farooq A, Pitz WJ, Sung C-J. Ignition of alkane-rich FACE gasoline fuels and their surrogate mixtures. Proc Combust Inst 2015;35:249-57.

[657] Axelbaum RL, Law CK, Flower WL. Preferential diffusion and concentration modification in sooting counterflow diffusion flames. Proc Combust Inst 1989;22:379-86.

[658] Law CK, Zhu DL, Yu G. Propagation and extinction of stretched premixed flames. Proc Combust Inst 1988;21:1419-26.

[659] Law CK. Dynamics of stretched flames. Proc Combust Inst 1989;22:1381-402.

[660] Tsuji H, Yamaoka I. The counterflow diffusion flame in the forward stagnation region of a porous cylinder. Proc Combust Inst 1967;11:979-84.

[661] Frenklach M. On the driving force of PAH production. Proc Combust Inst 1989;22:1075-82. [662] Liu FS, Ai YH, Kong WJ. Effect of hydrogen and helium addition to fuel on soot formation in an axisymmetric coflow laminar methane/air diffusion flame. International Journal of Hydrogen Energy 2014;39:3936-46.

[663] Guo HS, Liu FS, Smallwood GJ, Gülder ÖL. Numerical study on the influence of hydrogen addition on soot formation in a laminar ethylene-air diffusion flame. Combust Flame 2006;145:32438 .

[664] Gülder ÖL, Snelling DR, Sawchuk RA. Influence of hydrogen addition to fuel on temperature field and soot formation in diffusion flames. Proc Combust Inst 1996;26:2351-8.

[665] Trottier S, Guo H, Smallwood GJ, Johnson MR. Measurement and modeling of the sooting propensity of binary fuel mixtures. Proc Combust Inst 2007;31:611-9.

[666] Park S, Wang Y, Chung SH, Sarathy SM. A PAH growth mechanism and effect of alcohol addition on PAH formation in counterflow ethylene diffusion flames. 7th European Combustion Meeting. Budapest2015.

[667] Salamanca M, Sirignano M, Commodo M, Minutolo P, D’Anna A. The effect of ethanol on the particle size distributions in ethylene premixed flames. Exp Therm Fluid Sci 2012;43:71-5. [668] Roesler JF, Martinot S, McEnally CS, Pfefferle LD, Delfau JL, Vovelle C. Investigating the role of methane on the growth of aromatic hydrocarbons and soot in fundamental combustion processes. Combust Flame 2003;134:249-60.

[669] Sarathy SM, Farooq A, Kalghatgi GT. Recent progress in gasoline surrogate fuels. Prog Energy Combust Sci 2018;65:67-108. 
[670] Pitz WJ, Mueller CJ. Recent progress in the development of diesel surrogate fuels. Prog Energy Combust Sci 2011;37:330-50.

[671] Dooley S, Won SH, Jahangirian S, Ju YG, Dryer FL, Wang HW, Oehlschlaeger MA. The combustion kinetics of a synthetic paraffinic jet aviation fuel and a fundamentally formulated, experimentally validated surrogate fuel. Combust Flame 2012;159:3014-20.

[672] Dooley S, Won SH, Heyne J, Farouk TI, Ju YG, Dryer FL, Kumar K, Hui X, Sung CJ, Wang HW, Oehlschlaeger MA, Iyer V, Iyer S, Litzinger TA, Santoro RJ, Malewicki T, Brezinsky K. The experimental evaluation of a methodology for surrogate fuel formulation to emulate gas phase combustion kinetic phenomena. Combust Flame 2012;159:1444-66.

[673] Dooley S, Won SH, Chaos M, Heyne J, Ju YG, Dryer FL, Kumar K, Sung CJ, Wang HW, Oehlschlaeger MA, Santoro RJ, Litzinger TA. A jet fuel surrogate formulated by real fuel properties. Combust Flame 2010;157:2333-9.

[674] Violi A, Yan S, Eddings EG, Sarofim AF, Granata S, Faravelli T, Ranzi E. Experimental formulation and kinetic model for JP-8 surrogate mixtures. Combust Sci Technol 2002;174:399417.

[675] Hamins A, Anderson DT, Houston Miller J. Mechanistic studies of toluene destruction in diffusion flames. Combust Sci Technol 1990;71:175-95.

[676] Tosatto L, La Mantia B, Bufferand H, Duchaine P, Gomez A. Chemical structure of a methane counterflow diffusion flame perturbed with the addition of either JP-8 or a jet fuel surrogate. Proc Combust Inst 2009;32:1319-26.

[677] Bufferand H, Tosatto L, La Mantia B, Smooke MD, Gomez A. Experimental and computational study of methane counterflow diffusion flames perturbed by trace amounts of either jet fuel or a 6-component surrogate under non-sooting conditions. Combust Flame 2009;156:1594603.

[678] Das DD, St. John PC, McEnally CS, Kim S, Pfefferle LD. Measuring and predicting sooting tendencies of oxygenates, alkanes, alkenes, cycloalkanes, and aromatics on a unified scale.

Combust Flame 2018;190:349-64.

[679] Marchal C, Delfau JL, Vovelle C, Moreac G, Mounaim-Rousselle C, Mauss F. Modelling of aromatics and soot formation from large fuel molecules. Proc Combust Inst 2009;32:753-9.

[680] Mensch A, Santoro RJ, Litzinger TA, Lee SY. Sooting characteristics of surrogates for jet fuels. Combust Flame 2010;157:1097-105.

[681] Barrientos EJ, Lapuerta M, Boehman AL. Group additivity in soot formation for the example of C-5 oxygenated hydrocarbon fuels. Combust Flame 2013;160:1484-98.

[682] Yang Y, Boehman AL, Santoro RJ. A study of jet fuel sooting tendency using the threshold sooting index (TSI) model. Combust Flame 2007;149:191-205.

[683] Crossley SP, Alvarez WE, Resasco DE. Novel micropyrolyis index (MPI) to estimate the sooting tendency of fuels. Energ Fuel 2008;22:2455-64.

[684] Markatou P, Wang H, Frenklach M. A computational study of sooting limits in laminar premixed flames of ethane, ethylene, and acetylene. Combust Flame 1993;93:467-82.

[685] Takahashi F, Glassman I. Sooting correlations for premixed flames. Combust Sci Technol 1984;37:1-19.

[686] Watson RJ, Botero ML, Ness CJ, Morgan NM, Kraft M. An improved methodology for determining threshold sooting indices from smoke point lamps. Fuel 2013;111:120-30.

[687] Terry JB, Field E. The smoke tendency of refined kerosene and its determination. Industrial \& Engineering Chemistry Analytical Edition 1936;8:293-5.

[688] Li LM, Sunderland PB. An improved method of smoke point normalization. Combust Sci

Technol 2012;184:829-41.

[689] McEnally CS, Pfefferle LD. Improved sooting tendency measurements for aromatic hydrocarbons and their implications for naphthalene formation pathways. Combust Flame 2007;148:210-22. 
[690] McEnally CS, Pfefferle LD. Sooting tendencies of nonvolatile aromatic hydrocarbons. Proc Combust Inst 2009;32:673-9.

[691] McEnally CS, Xuan Y, St. John PC, Das DD, Jain A, Kim S, Kwan TA, Tan LK, Zhu J, Pfefferle LD. Sooting tendencies of co-optima test gasolines and their surrogates. Proc Combust Inst 2018.

[692] Montgomery MJ, Das DD, McEnally CS, Pfefferle LD. Analyzing the robustness of the yield sooting index as a measure of sooting tendency. Proc Combust Inst 2018.

[693] Perpignan AAV, Gangoli Rao A, Roekaerts DJEM. Flameless combustion and its potential towards gas turbines. Prog Energy Combust Sci 2018;69:28-62.

[694] Cho ES, Chung SH. Characteristics of NOx emission with flue gas dilution in air and fuel sides. KSME Int J 2004;18:2303-9.

[695] Merchan-Merchan W, Saveliev AV, Kennedy LA. Carbon nanostructures in opposed-flow methane oxy-flames. Combust Sci Technol 2003;175:2217-36.

[696] Silvestrini M, Merchan-Merchan W, Richter H, Saveliev A, Kennedy LA. Fullerene formation in atmospheric pressure opposed flow oxy-flames. Proc Combust Inst 2005;30:2545-52.

[697] Glassman, Yaccarino P. The effect of oxygen concentration on sooting diffusion flames.

Combust Sci Technol 1980;24:107-14.

[698] Lee KO, Megaridis CM, Zelepouga S, Saveliev AV, Kennedy LA, Charon O, Ammouri F. Soot formation effects of oxygen concentration in the oxidizer stream of laminar coannular nonpremixed methane/air flames. Combust Flame 2000;121:323-33.

[699] Axelbaum RL, Law CK. Soot formation and inert addition in diffusion flames. Proc Combust Inst 1991;23:1517-23.

[700] Kent JH, Wagner HG. Temperature and fuel effects in sooting diffusion flames. Proc Combust Inst 1985;20:1007-15.

[701] Law CK, Chung SH. Steady-state diffusion flame structure with Lewis number variations.

Combust Sci Technol 1982;29:129-45.

[702] Ishizuka S, Law CK. An experimental study on extinction and stability of stretched premixed flames. Proc Combust Inst 1982;19:327-35.

[703] Chung SH, Law CK. Burke-Schumann flame with streamwise and preferential diffusion. Combust Sci Technol 1984;37:21-46.

[704] McLintock IS. The effect of various diluents on soot production in laminar ethylene diffusion flames. Combust Flame 1968;12:217-25.

[705] Schug KP, Manheimertimnat Y, Yaccarino P, Glassman I. Sooting behavior of gaseous hydrocarbon diffusion flames and the influence of additives. Combust Sci Technol 1980;22:235-50. [706] Sun Z, Dally B, Nathan G, Alwahabi Z. Effects of hydrogen and nitrogen on soot volume fraction, primary particle diameter and temperature in laminar ethylene/air diffusion flames. Combust Flame 2017;175:270-82.

[707] Abian M, Millera A, Bilbao R, Alzueta MU. Effect of recirculation gases on soot formed from ethylene pyrolysis. Combust Sci Technol 2012;184:980-94.

[708] Cho ES, Chung SH. Numerical study on NO emission with flue gas dilution in air and fuel sides. J Mech Sci Technol 2005;19:1358-65.

[709] Annunziata A, Sirignano M, D'Anna A. The effect of carbon dioxide addition on soot formation in non-premixed ethylene flames. XXXV Meeting of the Italian Section of the Combustion Institute. Milano2012.

[710] Liu FS, Guo HS, Smallwood GJ, Gülder ÖL. The chemical effects of carbon dioxide as an additive in an ethylene diffusion flame: Implications for soot and NOx formation. Combust Flame 2001;125:778-87.

[711] Abian M, Millera A, Bilbao R, Alzueta MU. Experimental study on the effect of different $\mathrm{CO} 2$ concentrations on soot and gas products from ethylene thermal decomposition. Fuel 2012;91:307-12. 
[712] Teini PD, Karwat DMA, Atreya A. The effect of $\mathrm{CO} 2 / \mathrm{H} 2 \mathrm{O}$ on the formation of soot particles in the homogeneous environment of a rapid compression facility. Combust Flame 2012;159:1090-9. [713] McEnally CS, Pfefferle LD. The effects of premixing on soot production in nonpremixed flames fueled with unsaturated hydrocarbons. Proc Combust Inst 2002;29:2407-13.

[714] Gülder ÖL. Effects of oxygen on soot formation in methane, propane, and n-butane diffusion flames. Combust Flame 1995;101:302-10.

[715] Smyth KC, Tjossem PJH, Hamins A, Miller JH. Concentration measurements of $\mathrm{OH} \cdot$ and equilibrium analysis in a laminar methane-air diffusion flame. Combust Flame 1990;79:366-80.

[716] Rao VK, Bardon MF. The effect of water on gas phase soot formation in laminar diffusion flames. Combust Flame 1984;55:73-8.

[717] Liu FS, Consalvi JL, Fuentes A. Effects of water vapor addition to the air stream on soot formation and flame properties in a laminar coflow ethylene/air diffusion flame. Combust Flame 2014; 161:1724-34.

[718] Dec JE. A conceptual model of DI diesel combustion based on laser-sheet imaging. SAE Technical Paper 970873: SAE International; 1997.

[719] Dec JE. Advanced compression-ignition engines - understanding the in-cylinder processes. Proc Combust Inst 2009;32:2727-42.

[720] McEnally CS, Pfefferle LD. The effects of slight premixing on fuel decomposition and hydrocarbon growth in benzene-doped methane nonpremixed flames. Combust Flame 2002; 129:305-23.

[721] Hura HS, Glassman I. Soot formation in diffusion flames of fuel/oxygen mixtures. Proc Combust Inst 1989;22:371-8.

[722] Liu F, Thomson KA, Smallwood GJ. Experimental investigation of oxygen addition to fuel on soot formation in laminar coflow diffusion flames of ethylene and propane. ASME International Mechanical Engineering Congress and Exposition. Chicago, Illinois, USA2006.

[723] Chakraborty BB, Long R. The formation of soot and polycyclic aromatic hydrocarbons in diffusion flames III-Effect of additions of oxygen to ethylene and ethane respectively as fuels. Combust Flame 1968;12:469-76.

[724] Dearden P, Long R. Soot formation in ethylene and propane diffusion flames. Journal of Applied Chemistry 1968;18:243-51.

[725] Wright FJ. Effect of oxygen on the carbon-forming tendencies of diffusion flames. Fuel 1974;53:232-5.

[726] Wang Q, Legros G, Bonnety J, Morin C, Matynia A, Consalvi J-L, Liu F. Experimental assessment of the sudden-reversal of the oxygen dilution effect on soot production in coflow ethylene flames. Combust Flame 2017;183:242-52.

[727] Mitrovic A, Lee TW. Soot formation characteristics of laminar partially premixed flames. Combust Flame 1998;115:437-42.

[728] Wey C, Powell EA, Jagoda JI. The effect of temperature on the sooting behavior of laminar diffusion flames. Combust Sci Technol 1984;41:173-90.

[729] Bennett BAV, McEnally CS, Pfefferle LD, Smooke MD, Colket MB. Computational and experimental study of axisymmetric coflow partially premixed ethylene/air flames. Combust Flame 2001;127:2004-22.

[730] D'Anna A, D'Alessio A, Kent J. A computational study of hydrocarbon growth and the formation of aromatics in coflowing laminar diffusion flames of ethylene. Combust Flame 2001;125:1196-206.

[731] Nakamura Y, Ishii D, Satake S, Yamashita H. Slight-premixing effects on oxidation/formation of polycyclic aromatic hydrocarbon in counterflow flames. Journal of Propulsion and Power 2008;24:365-75.

[732] McEnally CS, Pfefferle LD. Experimental study of nonfuel hydrocarbon concentrations in coflowing partially premixed methane/air flames. Combust Flame 1999;118:619-32. 
[733] Xu W, Jiang Y, Qiu R, Ren X. Influence of halon replacements on laminar flame speeds and extinction limits of hydrocarbon flames. Combust Flame 2017;182:1-13.

[734] Pagliaro JL, Linteris GT, Babushok VI. Premixed flame inhibition by C2HF3Cl2 and C2HF5. Combust Flame 2016;163:54-65.

[735] Babushok VI, Linteris GT, Meier OC, Pagliaro JL. Flame inhibition by CF3CHCl2 (HCFC123). Combust Sci Technol 2014; 186:792-814.

[736] Ibiricu MM, Gaydon AG. Spectroscopic studies of the effect of inhibitors on counterflow diffusion flames. Combust Flame 1964;8:51-62.

[737] Frenklach M. Production of polycyclic aromatic hydrocarbons in chlorine containing environments. Combust Sci Technol 1990;74:283-96.

[738] Frenklach M, Hsu JP, Miller DL, Matula RA. Shock-tube pyrolysis of chlorinated hydrocarbons: Formation of soot. Combust Flame 1986;64:141-55.

[739] Senkan SM, Robinson JM, Gupta AK. Sooting Limits of Chlorinated-Hydrocarbon Methane Air Premixed Flames. Combust Flame 1983;49:305-14.

[740] Janssen JM, Senser DW. Incipient soot formation in dichloromethane-methane-air premixed flames. Combust Flame 1991;84:265-76.

[741] Garner FH, Long R, Graham AJ, Badakhshan A. The effect of certain halogenated methanes on pre-mixed and diffusion flames. Proc Combust Inst 1957;6:802-6.

[742] Morse JS, Cundy VA. Sooting in chlorinated hydrocarbon combustion-A critical review. Combust Sci Technol 1993;95:333-56.

[743] Leylegian JC, Zhu DL, Law CK, Wang H. Experiments and numerical simulation on the laminar flame speeds of dichloromethane and trichloromethane. Combust Flame 1998;114:285-93. [744] Leylegian JC, Sun HY, Law CK. Laminar flame speeds and kinetic modeling of hydrogen/chlorine combustion. Combust Flame 2005;143:199-210.

[745] Wang H, Hahn TO, Sung CJ, Law CK. Detailed oxidation kinetics and flame inhibition effects of chloromethane. Combust Flame 1996;105:291-307.

[746] McKinnon JT, Howard JB. Application of soot formation model: Effects of chlorine. Combust Sci Technol 1990;74:175-97.

[747] Gerasimov IE, Knyazkov DA, Shmakov AG, Paletsky AA, Shvartsberg VM, Bolshova TA, Korobeinichev OP. Inhibition of hydrogen-oxygen flames by iron pentacarbonyl at atmospheric pressure. Proc Combust Inst 2011;33:2523-9.

[748] Rumminger MD, Reinelt D, Babushok V, Linteris GT. Numerical study of the inhibition of premixed and diffusion flames by iron pentacarbonyl. Combust Flame 1999;116:207-19.

[749] Linteris GT, Rumminger MD, Babushok VI. Catalytic inhibition of laminar flames by transition metal compounds. Prog Energy Combust Sci 2008;34:288-329.

[750] Shmakov AG, Korobeinichev OP, Knyazkov DA, Paletsky AA, Maksutov RA, Gerasimov

IE, Bolshova TA, Kiselev VG, Gritsan NP. Combustion chemistry of $\mathrm{Ti}\left(\mathrm{OC}_{3} \mathrm{H}_{7}\right)_{4}$ in premixed flat burner-stabilized $\mathrm{H}_{2} / \mathrm{O}_{2} / \mathrm{Ar}$ flame at 1 atm. Proc Combust Inst 2013;34:1143-9.

[751] Li S, Ren Y, Biswas P, Tse SD. Flame aerosol synthesis of nanostructured materials and functional devices: Processing, modeling, and diagnostics. Prog Energy Combust Sci 2016;55:1-59. [752] Pratsinis SE. Flame aerosol synthesis of ceramic powders. Prog Energy Combust Sci 1998;24:197-219.

[753] Vander Wal RL. Fe-catalyzed single-walled carbon nanotube synthesis within a flame environment. Combust Flame 2002;130:37-47.

[754] Celnik M, West R, Morgan N, Kraft M, Moisala A, Wen J, Green W, Richter H. Modelling gas-phase synthesis of single-walled carbon nanotubes on iron catalyst particles. Carbon 2008;46:422-33.

[755] Wen JZ, Goldsmith CF, Ashcraft RW, Green WH. Detailed kinetic modeling of iron nanoparticle synthesis from the decomposition of $\mathrm{Fe}(\mathrm{CO})_{5}$. J Phys Chem C 2007;111:5677-88. 
[756] Simonsson J, Olofsson N-E, Bladh H, Sanati M, Bengtsson P-E. Influence of potassium and iron chloride on the early stages of soot formation studied using imaging LII/ELS and TEM techniques. Proc Combust Inst 2017;36:853-60.

[757] Kim KB, Masiello KA, Hahn DW. Reduction of soot emissions by iron pentacarbonyl in isooctane diffusion flames. Combust Flame 2008;154:164-80.

[758] Nash DG, Swanson NB, Preston WT, Yelverton TLB, Roberts WL, Wendt JOL, Linak WP. Environmental implications of iron fuel borne catalysts and their effects on diesel particulate formation and composition. J Aerosol Sci 2013;58:50-61.

[759] Marsh ND, Preciado I, Eddings EG, Sarofim AF, Palotas AB, Robertson JD. Evaluation of organometallic fuel additives for soot supression. Combust Sci Technol 2007;179:987-1001.

[760] Simonsson J, Olofsson N-E, Hosseinnia A, Bengtsson P-E. Influence of potassium chloride and other metal salts on soot formation studied using imaging LII and ELS, and TEM techniques. Combust Flame 2018;190:188-200.

[761] Hirasawa T, Sung CJ, Yang ZW, Joshi A, Wang H. Effect of ferrocene addition on sooting limits in laminar premixed ethylene-oxygen-argon flames. Combust Flame 2004;139:288-99. [762] Agafonov GL, Smirnov VN, Vlasov PA. Effect of iron pentacarbonyl on soot formation behind shock waves. Combust Sci Technol 2012;184:1838-61.

[763] Ritrievi KE, Longwell JP, Sarofim AF. The effects of ferrocene addition on soot particle inception and growth in premixed ethylene flames. Combust Flame 1987;70:17-31.

[764] Hahn DW, Charalampopoulos TT. The role of iron additives in sooting premixed flames. Proc Combust Inst 1992;24:1007-14.

[765] Zhang J, Megaridis CM. Soot suppression by ferrocene in laminar ethylene/air nonpremixed flames. Combust Flame 1996;105:528-40.

[766] Hu C, Li W, Lin Q, Cheng X, Huang Q, Zhang H, Wang Z. Effects of ferrocene on flame temperature, formation of soot particles and growth of polycyclic aromatic hydrocarbons. J Energy Inst 2017;90:893-901.

[767] Kim SH, Fletcher RA, Zachariah MR. Understanding the difference in oxidative properties between flame and diesel soot nanoparticles: The role of metals. Environ Sci Technol 2005;39:4021-6.

[768] Kim K, Hahn DW. Interaction between iron based compound and soot particles in diffusion flame. Energy 2016;116:933-41.

[769] Raj A, Croiset E, Wen JZ. Numerical analysis of effects of iron pentacarbonyl as fuel additive for reducing NO and soot precursors from methane/air diffusion flame. Fuel 2018;216:768-80. [770] Böhm H, Feldermann C, Heidermann T, Jander H, Lüers B, Wagner HG. Soot formation in premixed $\mathrm{C}_{2} \mathrm{H}_{4}$-air flames for pressures up to 100 bar. Proc Combust Inst 1992;24:991-8.

[771] McArragher JS, Tan KJ. Soot formation at high pressures: A literature review. Combust Sci Technol 1972;5:257-61.

[772] Böhm H, Hesse D, Jander H, Lüers B, Pietscher J, Wagner HGG, Weiss M. The influence of pressure and temperature on soot formation in premixed flames. Proc Combust Inst 1989;22:40311.

[773] Mätzing H, Wagner HG. Measurements about the influence of pressure on carbon formation in premixed laminar $\mathrm{C}_{2} \mathrm{H}_{4}$-air flames. Proc Combust Inst 1988;21:1047-55.

[774] Hanisch S, Jander H, Pape T, Wagner HG. Soot mass growth and coagulation of soot particles in C2H4/air-flames at 15 bar. Proc Combust Inst 1994;25:577-84.

[775] Braun-Unkhoff M, Chrysostomou A, Frank P, Gutheil E, Luckerath R, Stricker W.

Experimental and numerical study on soot formation in laminar high-pressure flames. Symp (Int) Combust 1998:1565-72.

[776] Heidermann T, Jander H, Gg. Wagner H. Soot particles in premixed $\mathrm{C}_{2} \mathrm{H}_{4}$-air-flames at high pressures ( $\mathrm{P}=30-70$ bar). Phys Chem Chem Phys 1999;1:3497-502. 
[777] Tsurikov MS, Geigle KP, Krüger V, Schneider-Kühnle Y, Stricker W, Lückerath R, Hadef R, Aigner M. Laser-based investigation of soot formation in laminar premixed flames at atmospheric and elevated pressures. Combust Sci Technol 2005;177:1835-62.

[778] Mauss F, Bockhorn H. Soot formation in premixed hydrocarbon flames - Prediction of temperature and pressure-dependence. Z Phys Chem 1995;188:45-60.

[779] Roper FG. The prediction of laminar jet diffusion flame sizes: Part I. Theoretical model. Combust Flame 1977;29:219-26.

[780] Roper FG, Smith C, Cunningham AC. Prediction of laminar jet diffusion flame sizes .2. Experimental verification. Combust Flame 1977;29:227-34.

[781] Mandatori PM, Gülder ÖL. Soot formation in laminar ethane diffusion flames at pressures from 0.2 to 3.3 MPa. Proc Combust Inst 2011;33:577-84.

[782] Charest MRJ, Groth CPT, Gülder ÖL. A numerical study on the effects of pressure and gravity in laminar ethylene diffusion flames. Combust Flame 2011;158:1933-45.

[783] Charest MRJ, Joo HI, Gülder ÖL, Groth CPT. Experimental and numerical study of soot formation in laminar ethylene diffusion flames at elevated pressures from 10 to $35 \mathrm{~atm}$. Proc Combust Inst 2011;33:549-57.

[784] Liu F, Thomson KA, Guo H, Smallwood GJ. Numerical and experimental study of an axisymmetric coflow laminar methane-air diffusion flame at pressures between 5 and 40 atmospheres. Combust Flame 2006;146:456-71.

[785] Griffin EA, Christensen M, Gülder ÖL. Effect of ethanol addition on soot formation in laminar methane diffusion flames at pressures above atmospheric. Combust Flame 2018;193:30612.

[786] Zhou L, Xiong G, Zhang M, Chen L, Ding S, de Goey LPH. Experimental study of polycyclic aromatic hydrocarbons (PAHs) in n-heptane laminar diffusion flames from1.0 to 3.0 bar. Fuel 2017;209:265-73.

[787] Zhou L, Dam NJ, Boot MD, de Goey LPH. Measurements of sooting tendency in laminar diffusion flames of n-heptane at elevated pressure. Combust Flame 2013;160:2507-16.

[788] Figura L, Gomez A. Laminar counterflow steady diffusion flames under high pressure $(\mathrm{P}<=3 \mathrm{MPa})$ conditions. Combust Flame 2012;159:142-50.

[789] Sun CJ, Sung CJ, hu DL, Law CK. Response of counterflow premixed and diffusion flames to strain rate variations at reduced and elevated pressures. Proc Combust Inst 1996;26:1111-20. [790] Ma B, Wang GH, Magnotti G, Barlow RS, Long MB. Intensity-ratio and color-ratio thinfilament pyrometry: Uncertainties and accuracy. Combust Flame 2014;161:908-16.

[791] Narayanaswamy K, Blanquart G, Pitsch H. A consistent chemical mechanism for oxidation of substituted aromatic species. Combust Flame 2010;157:1879-98.

[792] Harris MM, King GB, Laurendeau NM. Response to comments and errata, "Influence of temperature and hydroxyl concentration on incipient soot formation in premixed flames". Combust Flame 1987;67:269-72.

[793] Takahashi F, Glassman I. Influence of temperature and hydroxyl concentration on incipient soot formation in premixed flames - Comments. Combust Flame 1987;67:267-8.

[794] Ciajolo A, D'Anna A, Barbella R, Tregrossi A, Violi A. The effect of temperature on soot inception in premixed ethylene flames. Twenty-Sixth Symposium (International) on Combustion, Vols 1 and 2 1996:2327-33.

[795] Alfe M, Apicella B, Rouzaud JN, Tregrossi A, Ciajolo A. The effect of temperature on soot properties in premixed methane flames. Combust Flame 2010;157:1959-65.

[796] Gomez A, Sidebotham G, Glassman I. Sooting Behavior in Temperature-Controlled Laminar Diffusion Flames. Combust Flame 1984;58:45-57.

[797] Santoro RJ, Semerjian HG. Soot formation in diffusion flames: Flow rate, fuel species and temperature effects. Proc Combust Inst 1985;20:997-1006. 
[798] Frenklach M, Taki S, Durgaprasad MB, Matula RA. Soot formation in shock-tube pyrolysis of acetylene, allene, and 1,3-butadiene. Combust Flame 1983;54:81-101.

[799] Poinsot T, Veynante D. Theoretical and numerical combustion. 2nd ed. Philadelphia:

Edwards; 2005.

[800] Bohm H, Lacas F. Evaluating the strain and fuel concentration of counterflow diffusion

flames by selected species. Progress in Computational Fluid Dynamics 2005;5:420-5.

[801] Decroix ME, Roberts WL. Transient flow field effects on soot volume fraction in diffusion flames. Combust Sci Technol 2000;160:165-89.

[802] Haworth DC, Drake MC, Pope SB, Blint RJ. The importance of time-dependent flame structures in stretched laminar flamelet models for turbulent jet diffusion flames. Proc Combust Inst 1989;22:589-97.

[803] Im HG, Bechtold JK, Law CK. Counterflow diffusion flames with unsteady strain rates. Combust Sci Technol 1995;106:345-61.

[804] Im HG, Law CK, Kim JS, Williams FA. Response of counterflow diffusion flames to oscillating strain rates. Combust Flame 1995;100:21-30.

[805] Egolfopoulos FN, Campbell CS. Unsteady counterflowing strained diffusion flames:

Diffusion-limited frequency response. J Fluid Mech 1996;318:1-29.

[806] Saitoh T, Otsuka Y. Unsteady behavior of diffusion flames and premixed flames for counterflow geometry. Combust Sci Technol 1976;12:135-46.

[807] Ghoniem AF, Soteriou MC, Knio OM, Cetegen B. Effect of steady and periodic strain on unsteady flamelet combustion. Proc Combust Inst 1992;24:223-30.

[808] Darabiha N. Transient behaviour of laminar counterflow hydrogen-air diffusion flames with complex chemistry. Combust Sci Technol 1992;86:163-81.

[809] Cetegen BM, Bogue DR. Combustion in a stretched fuel strip with finite rate chemistry.

Combust Flame 1991;86:359-70.

[810] Valiev DM, Zhu M, Bansal G, Kolla H, Law CK, Chen JH. Pulsating instability of externally forced premixed counterflow flame. Combust Flame 2013;160:285-94.

[811] Welle EJ, Roberts WL, Decroix ME, Carter CD, Donbar JM. Simultaneous particle-imaging velocimetry and $\mathrm{OH}$ planar laser-induced fluorescence measurements in an unsteady counterflow propane/air diffusion flame. Proc Combust Inst 2000;28:2021-7.

[812] Welle EJ, Roberts WL, Carter CD, Donbar JM. The response of a propane-air counter-flow diffusion flame subjected to a transient flow field. Combust Flame 2003;135:285-97.

[813] Li SC. Spray stagnation flames. Prog Energy Combust Sci 1997;23:303-47.

[814] Darabiha N, Lacas F, Rolon JC, Candel S. Laminar counterflow spray diffusion flames: A comparison between experimental results and complex chemistry calculations. Combust Flame 1993;95:261-75.

[815] Santoro VS, Kyritsis DC, Gomez A. An experimental study of vortex-flame interaction in counterflow spray diffusion flames. Proc Combust Inst 2000;28:1023-30.

[816] Chen G, Gomez A. Counterflow diffusion flames of quasi-monodisperse electrostatic sprays. Proc Combust Inst 1992;24:1531-9.

[817] Orain M, Hardalupas Y. Droplet characteristics and local equivalence ratio of reacting mixture in spray counterflow flames. Exp Therm Fluid Sci 2014;57:261-74.

[818] Chen NH, Rogg B, Bray KNC. Modelling laminar two-phase counterflow flames with detailed chemistry and transport. Proc Combust Inst 1992;24:1513-21.

[819] Watanabe H, Kurose R, Hwang S-M, Akamatsu F. Characteristics of flamelets in spray flames formed in a laminar counterflow. Combust Flame 2007;148:234-48.

[820] Nakamura M, Akamatsu F, Kurose R. Combustion mechanism of liquid fuel spray in a gaseous flame. Physics of Fluids 2005;17:123301.

[821] Luo Y, Wen X, Wang H, Luo K, Fan J. Evaluation of different flamelet tabulation methods for laminar spray combustion. Physics of Fluids 2018;30:053603. 
[822] Greenberg JB, Alibagli D, Tambour Y. An opposed jet quasi-monodisperse spray diffusion flame. Combust Sci Technol 1986;50:255-70.

[823] Greenberg JB, Sarig N. Polydispersity and reaction zone structure in partially premixed spray flames. Proc Combust Inst 1996;26:1705-11.

[824] Dvorjetski A, Greenberg JB. Steady-state and extinction analyses of counterflow spray diffusion flames with arbitrary finite evaporation rate. Combust Sci Technol 2002;174:187-208.

[825] Bossard JA, Peck RE. Droplet size distribution effects in spray combustion. Proc Combust Inst 1996;26:1671-7.

[826] Continillo G, Sirignano WA. Counterflow spray combustion modeling. Combust Flame 1990;81:325-40.

[827] Tsushima S, Saitoh H, Akamatsu F, Katsuki M. Observation of combustion characteristics of droplet clusters in a premixed-spray flame by simultaneous monitoring of planar spray images and local chemiluminescence. Proc Combust Inst 1998;27:1967-74.

[828] Chiu HH, Kim HY, Croke EJ. Internal group combustion of liquid droplets. Proc Combust Inst 1982;19:971-80.

[829] Chiu HH. Advances and challenges in droplet and spray combustion. I. Toward a unified theory of droplet aerothermochemistry. Prog Energy Combust Sci 2000;26:381-416.

[830] Fialkov AB. Investigations on ions in flames. Prog Energy Combust Sci 1997;23:399-528.

[831] Lawton J, Weinberg FJ. Electrical aspects of combustion. Oxford, UK: Clarenton Press; 1969.

[832] Borgatelli F, Dunn-Rankin D. Behavior of a small diffusion flame as an electrically active component in a high-voltage circuit. Combust Flame 2012;159:210-20.

[833] Fialkov AB, Dennebaum J, Homann KH. Large molecules, ions, radicals, and small soot particles in fuel-rich hydrocarbon flames Part V: Positive ions of polycyclic aromatic hydrocarbons (PAH) in low-pressure premixed flames of benzene and oxygen. Combust Flame 2001;125:763-77. [834] Gerhardt P, Löffler S, Homann KH. Polyhedral carbon ions in hydrocarbon flames. Chem Phys Lett 1987;137:306-10.

[835] Gerhardt P, Löffler S, Homann KH. The formation of polyhedral carbon ions in fuel-rich acetylene and benzene flames. Proc Combust Inst 1989;22:395-401.

[836] Homann KH, Wolf H. Charged particles in sooting flames II. Determination of the fraction of charged soot. Berichte der Bunsengesellschaft für physikalische Chemie 1983;87:1073-7.

[837] Maricq MM. Size and charge of soot particles in rich premixed ethylene flames. Combust Flame 2004;137:340-50.

[838] Karnani S, Dunn-Rankin D. Detailed characterization of DC electric field effects on small non-premixed flames. Combust Flame 2015;162:2865-72.

[839] Hu J, Rivin B, Sher E. The effect of an electric field on the shape of co-flowing and candletype methane-air flames. Exp Therm Fluid Sci 2000;21:124-33.

[840] Kuhl J, Seeger T, Zigan L, Will S, Leipertz A. On the effect of ionic wind on structure and temperature of laminar premixed flames influenced by electric fields. Combust Flame 2017;176:391-9.

[841] Won SH, Cha MS, Park CS, Chung SH. Effect of electric fields on reattachment and propagation speed of tribrachial flames in laminar coflow jets. Proc Combust Inst 2007;31:963-70. [842] Kim MK, Chung SH, Kim HH. Effect of AC electric fields on the stabilization of premixed bunsen flames. Proc Combust Inst 2011;33:1137-44.

[843] Kim MK, Chung SH, Kim HH. Effect of electric fields on the stabilization of premixed laminar bunsen flames at low AC frequency: Bi-ionic wind effect. Combust Flame 2012;159:11519.

[844] Tran M-V, Cha MS. Time evolution of propagating nonpremixed flames in a counterflow, annular slot burner under AC electric fields. Proc Combust Inst 2017;36:1421-30.

[845] Sánchez-Sanz M, Murphy DC, Fernandez-Pello C. Effect of an external electric field on the propagation velocity of premixed flames. Proc Combust Inst 2015;35:3463-70. 
[846] Won SH, Ryu SK, Kim MK, Cha MS, Chung SH. Effect of electric fields on the propagation speed of tribrachial flames in coflow jets. Combust Flame 2008;152:496-506.

[847] Cha MS, Lee SM, Kim KT, Chung SH. Soot suppression by nonthermal plasma in coflow jet diffusion flames using a dielectric barrier discharge. Combust Flame 2005;141:438-47.

[848] Kono M, Carleton FB, Jones AR, Weinberg FJ. The effect of nonsteady electric fields on sooting flames. Combust Flame 1989;78:357-64.

[849] Kono M, Iinuma K, Kumagai S. The effect of DC to $10 \mathrm{MHz}$ electric field on flame luminosity and carbon formation. Proc Combust Inst 1981;18:1167-74.

[850] Xie L, Kishi T, Kono M. Investigation on the effect of electric fields on soot formation and flame structure of diffusion flames. Proc Combust Inst 1992;24:1059-66.

[851] Carleton FB, Weinberg FJ. Electric field-induced flame convection in the absence of gravity. Nature 1987;330:635.

[852] Jones FL, Becker PM, Heinsohn RJ. A mathematical model of the opposed-jet diffusion flame: Effect of an electric field on concentration and temperature profiles. Combust Flame 1972;19:351-62.

[853] Egolfopoulos FN, Hansen N, Ju Y, Kohse-Höinghaus K, Law CK, Qi F. Advances and challenges in laminar flame experiments and implications for combustion chemistry. Prog Energy Combust Sci 2014;43:36-67.

[854] Veloo PS, Egolfopoulos FN. Studies of n-propanol, iso-propanol, and propane flames. Combust Flame 2011;158:501-10.

[855] Niemann U, Seshadri K, Williams FA. Methane, ethane, and ethylene laminar counterflow diffusion flames at elevated pressures: Experimental and computational investigations up to 2.0 MPa. Combust Flame 2014;161:138-46.

[856] Lee S, Ha H, Dunn-Rankin D, Kwon OC. Effects of pressure on structure and extinction limits of counterflow nonpremixed water-laden methane/air flames. Energy 2017;134:545-53. [857] Liu F, Smallwood GJ, Gülder ÖL, Ju Y. Asymptotic analysis of radiative extinction in counterflow diffusion flames of nonunity Lewis numbers. Combust Flame 2000;121:275-87. [858] Maruta K, Yoshida M, Guo H, Ju Y, Niioka T. Extinction of low-stretched diffusion flame in microgravity. Combust Flame 1998;112:181-7.

[859] Wang HY, Chen WH, Law CK. Extinction of counterflow diffusion flames with radiative heat loss and nonunity Lewis numbers. Combust Flame 2007;148:100-16.

[860] Oh T-K, Lee JS, Chung SH. Effect of gas-phase and surface radiation on the structure and extinction of diffusion flames stabilized on a condensed fuel. Int J Heat Mass Tran 1994;37:2893900.

[861] Hall RJ, Smooke MD, Colket MB. Predictions of soot dynamics in opposed jet diffusion flames: Gordon \& Breach; 1996.

[862] Turns SR, Myhr FH. Oxides of nitrogen emissions from turbulent jet flames: Part I-Fuel effects and flame radiation. Combust Flame 1991;87:319-35.

[863] Naik SV, Laurendeau NM. Quantitative laser-saturated fluorescence measurements of nitric oxide in counter-flow diffusion flames under sooting oxy-fuel conditions. Combust Flame 2002;129:112-9.

[864] Guo HS, Liu FS, Smallwood GJ. Soot and NO formation in counterflow ethylene/oxygen/nitrogen diffusion flames. Combust Theor Model 2004;8:475-89.

[865] Wang H. Ten questions concerning mechanisms and models of soot formation. International Bunsen Discussion Meeting: Chemistry and Diagnostics for Clean Combustion. Bielefeld, Germany2017.

[866] Hessler JP, Seifert S, Winans RE. Spatially resolved small-angle x-ray scattering studies of soot inception and growth. Proc Combust Inst 2002;29:2743-8.

[867] Wang H, Zhao B, Wyslouzil B, Streletzky K. Small-angle neutron scattering of soot formed in laminar premixed ethylene flames. Proc Combust Inst 2002;29:2749-57. 
[868] Liu G, Liu D. Direct simultaneous reconstruction for temperature and concentration profiles of soot and metal-oxide nanoparticles in nanofluid fuel flames by a CCD camera. Int J Heat Mass Tran 2018;124:564-75.

[869] Liu G, Liu D. Inverse radiation analysis for simultaneous reconstruction of temperature and volume fraction fields of soot and metal-oxide nanoparticles in a nanofluid fuel sooting flame. Int $\mathrm{J}$ Heat Mass Tran 2018;118:1080-9. 